\title{
Asymmetric Organocatalyzed Reaction Sequence to Synthesize Chiral Bridged and Spiro-Bridged Benzofused Aminals via Divergent Pathways
}

Ying-Han Chen, Xue-Jiao Lv, Zhi-Hao You, and Yan-Kai Liu*

E-mail: liuyankai@ouc.edu.cn

Supporting Information 


\section{Table of Contents}

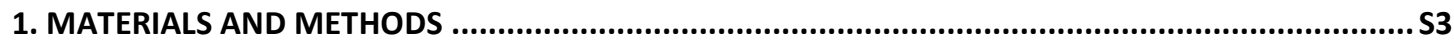

2. GENERAL PROCEDURES FOR CASCADE REACTIONS ............................................................S4

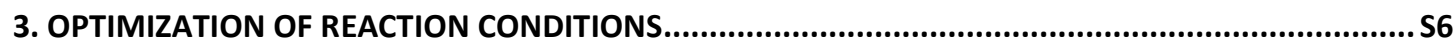

4. CONTROL EXPERIMENTS AND THE IDENTIFICATION OF REACTION INTERMEDIATES ....................S7

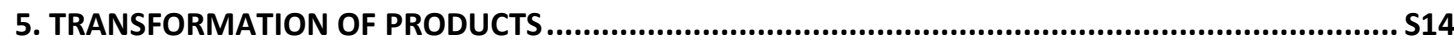

6. SINGLE CRYSTAL X-RAY DIFFRACTION DATA FOR COMPOUNDS ........................................... S23

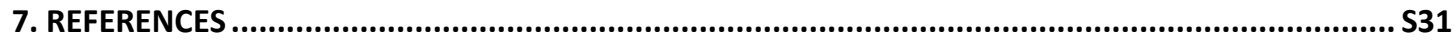

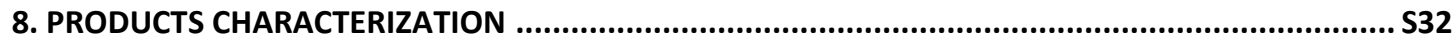

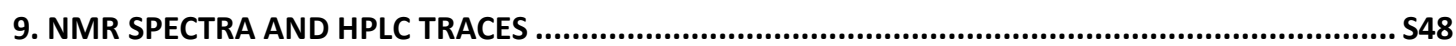




\section{Materials and methods}

All commercial available compounds were purchased from Adamas-beta , Alfa Aesar, Fluka, J\&K, Energy, Aladdin, Meryer, Macklin and Bepharm. Catalysts 3a, 3b and 3c were purchased from Daicel. The substituted 2-hydroxy cinnamaldehydes and cyclic $\mathrm{N}$-sulfonyl ketimines were synthesized according to literature procedure. ${ }^{[1,2,3]}$ NMR spectra were recorded on Agilient DD2 $500\left(500 \mathrm{MHz}\right.$ for ${ }^{1} \mathrm{H}$ and at $125 \mathrm{MHz}$ for $\left.{ }^{13} \mathrm{C}\right)$. The chemical shifts $(\delta)$ for ${ }^{1} \mathrm{H}$ and ${ }^{13} \mathrm{C}$ are given in ppm relative to $\mathrm{CDCl}_{3}(7.26$ ppm for ${ }^{1} \mathrm{H}$ and 77.16 ppm for ${ }^{13} \mathrm{C}$ ) and $d_{6}$-DMSO (2.50 ppm for ${ }^{1} \mathrm{H}$ and $39.52 \mathrm{ppm}$ for ${ }^{13} \mathrm{C}$ ). High-resolution mass spectra (HRMS) were obtained from the Waters Q-Tof Ultima Global. X-ray data were obtained from Zhongke chemical technology service center. Optical rotations were reported as follows: $[\mathrm{a}]_{\mathrm{D}}{ }^{20}$ (c in g per $100 \mathrm{~mL}$, solvent). Chromatographic purifications were accomplished on silica gel (300-400 mesh). Thin layer chromatography (TLC) analyses were run on silica gel plates purchased from Merck (silica gel 60, GF254). HPLC analyses on chiral stationary phase were performed on HITACHI Chromaste. Daicel Chiralpak IA, IB, IC columns with $i-\mathrm{PrOH} / n$-hexane $/ \mathrm{CH}_{2} \mathrm{Cl}_{2}$ as the eluents were used. 


\section{General procedures for cascade reactions}

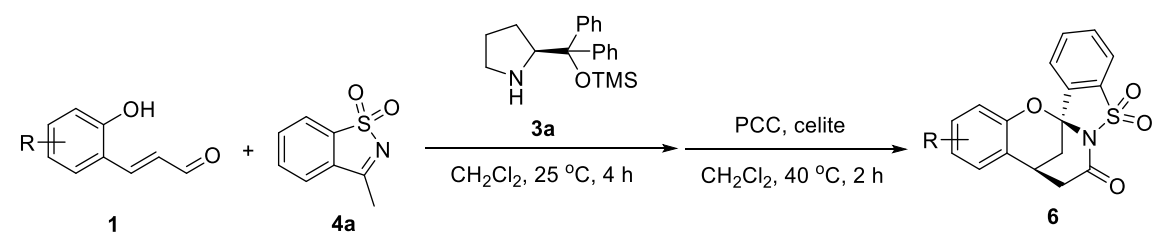

A glass vial equipped with a magnetic stirring bar was charged with $1(0.11 \mathrm{mmol}, 1.1$ equiv) and $3 a$ ( $6.5 \mathrm{mg}, 20 \mathrm{~mol} \%)$ in dichloromethane $(200 \mu \mathrm{L}, 0.5 \mathrm{M})$ to afford a dark red solution, and then $4 \mathrm{a}(18.0 \mathrm{mg}, 0.10 \mathrm{mmol}, 1.0$ equiv) was added. The reaction mixture was stirred at $25{ }^{\circ} \mathrm{C}$ for $4 \mathrm{~h}$ and it turned into an orange solution. After completion of the addition, another $800 \mu \mathrm{L}$ dichloromethane was added, followed by pyridinium chlorochromate (PCC, $64.0 \mathrm{mg}, 3.0$ equiv) and $64.0 \mathrm{mg}$ celite, and then the brown reaction mixture was stirred at $40{ }^{\circ} \mathrm{C}$ for another $2 \mathrm{~h}$. The product 6 was purified with chromatographic column (petroleum ether : ethyl acetate 2:1).

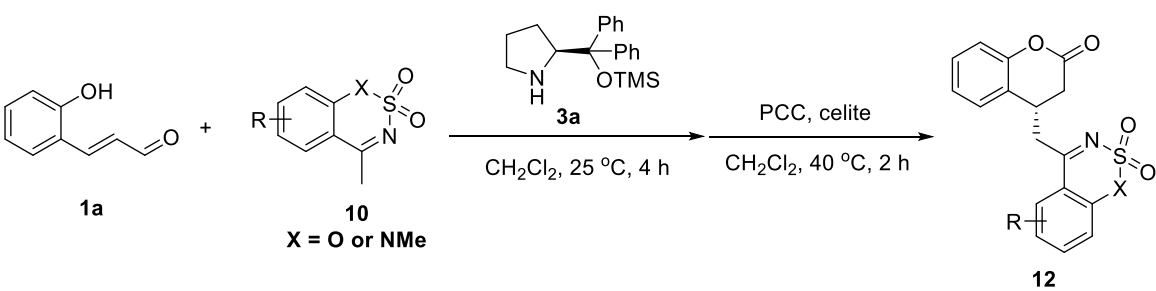

A glass vial equipped with a magnetic stirring bar was charged with 1 a $(16.3 \mathrm{mg}, 0.11$ mmol, 1.1 equiv) and $3 \mathrm{a}(6.5 \mathrm{mg}, 20 \mathrm{~mol} \%)$ in dichloromethane $(200 \mu \mathrm{L}, 0.5 \mathrm{M})$ to afford a dark red solution, and then $10(0.10 \mathrm{mmol}, 1.0$ equiv) was added. The reaction mixture was stirred at $25{ }^{\circ} \mathrm{C}$ for $4 \mathrm{~h}$ (the reaction with $10 \mathrm{~g}$ when $\mathrm{X}=\mathrm{NMe}$ was conducted under $40{ }^{\circ} \mathrm{C}$ for $24 \mathrm{~h}$ ) and it turned into yellow solution. After completion of the addition, another $800 \mu \mathrm{L}$ dichloromethane, pyridinium chlorochromate (PCC, $64.0 \mathrm{mg}, 3.0$ equiv) and $64.0 \mathrm{mg}$ celite were added sequentially, and then the reaction mixture was stirred at $40{ }^{\circ} \mathrm{C}$ for another $2 \mathrm{~h}$. The product 12 was purified with chromatographic column (petroleum ether : ethyl acetate : dichloromethane 3:1:1). 


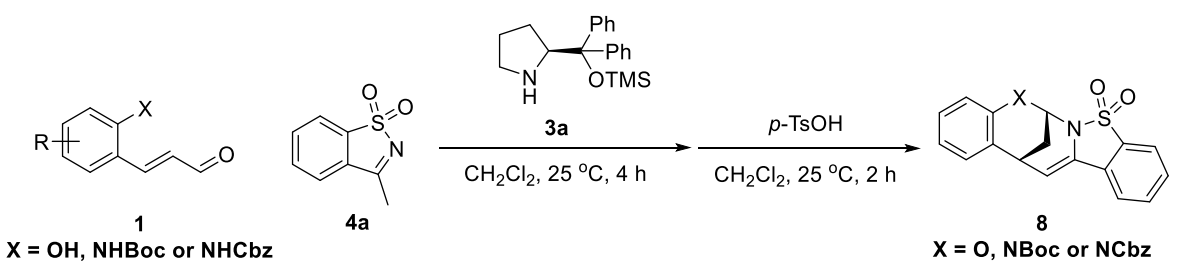

A glass vial was charged with 1 (0.11 mmol, 1.1 equiv) and $3 a(6.5 \mathrm{mg}, 20 \mathrm{~mol} \%)$ in dichloromethane $(200 \mu \mathrm{L}, 0.5 \mathrm{M})$, then $4 \mathrm{a}(18.0 \mathrm{mg}, 0.10 \mathrm{mmol}, 1.0$ equiv) was added after the reaction mixture became dark red. Keep reacting at $25{ }^{\circ} \mathrm{C}$ for $4 \mathrm{~h}$, another $800 \mu \mathrm{L}$ dichloromethane and $p$-toluenesulfonic acid $(p$ - $\mathrm{TsOH}, 7.3 \mathrm{mg}, 0.4$ equiv) were added. After the reaction was completed, the reaction mixture was purified through chromatographic column (petroleum ether : ethyl acetate 5:1). The crude product was suspended in methanol and then filtered to afford pure product 8 as white solid.

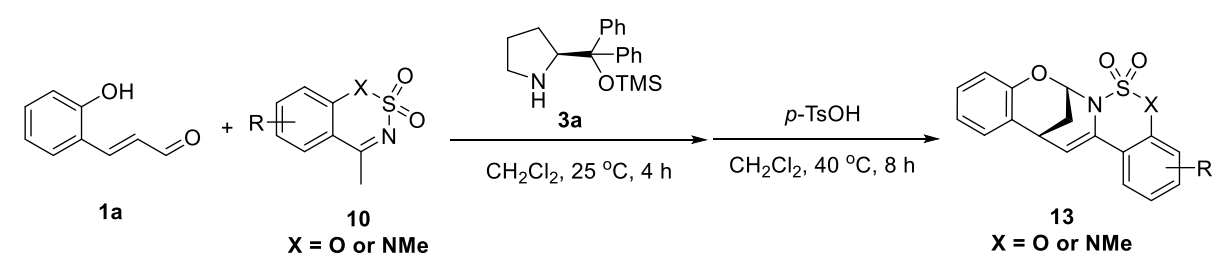

A glass vial was charged with 1a (16.3 mg, $0.11 \mathrm{mmol}, 1.1$ equiv) and 3a (6.5 mg, 20 mol \%) in dichloromethane $(200 \mu \mathrm{L}, 0.5 \mathrm{M})$, then 10 (0.10 mmol, 1.0 equiv) was added after the reaction mixture became dark red. Keep reacting at $25{ }^{\circ} \mathrm{C}$ for $4 \mathrm{~h}$ (the reaction with $10 \mathrm{~g}$ when $\mathrm{X}=\mathrm{NMe}$ was conducted under $40{ }^{\circ} \mathrm{C}$ for $24 \mathrm{~h}$ ), then another $800 \mu \mathrm{L}$ dichloromethane and $p$-toluenesulfonic acid ( $p$-TsOH, $18.3 \mathrm{mg}, 1.0$ equiv) were added and the reaction mixture was keep stirring at $40{ }^{\circ} \mathrm{C}$ for $8 \mathrm{~h}$. After the reaction was completed, the reaction mixture was purified through chromatographic column (petroleum ether : ethyl acetate 5:1). The crude product was suspended in methanol, then filter it to afford pure product 13 as white solid. 


\section{Optimization of reaction conditions}

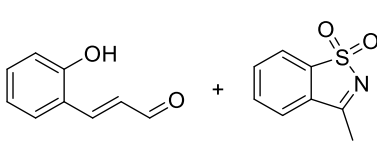

$1 \mathrm{a}$

$4 a$
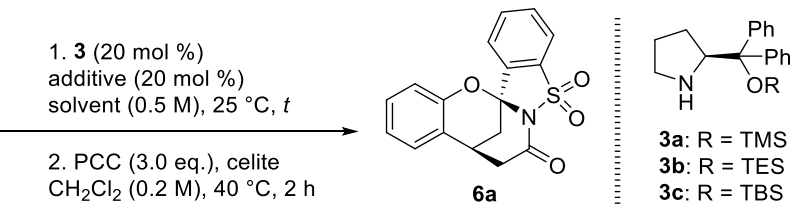

Table S1. Optimization of the enantioselective cascade reactions ${ }^{a}$.

\begin{tabular}{lllllll}
\hline entry & catalyst & additive & solvent & $\mathrm{t}(\mathrm{h})$ & ${\text { yield }(\%)^{b}}^{b}$ & ee $(\%)^{c}$ \\
\hline 1 & $3 \mathbf{a}$ & $\mathrm{PhCOOH}$ & $\mathrm{CH}_{2} \mathrm{Cl}_{2}$ & 5 & 50 & 96 \\
2 & $3 \mathbf{b}$ & $\mathrm{PhCOOH}$ & $\mathrm{CH}_{2} \mathrm{Cl}_{2}$ & 5 & 53 & 98 \\
3 & $3 \mathbf{c}$ & $\mathrm{PhCOOH}$ & $\mathrm{CH}_{2} \mathrm{Cl}_{2}$ & 5 & 47 & 98 \\
\hline 4 & $3 \mathbf{a}$ & - & $\mathrm{CH}_{2} \mathrm{Cl}_{2}$ & 4 & 66 & 97 \\
5 & $3 \mathbf{a}$ & $p-\mathrm{NO}_{2} \mathrm{C}_{6} \mathrm{H}_{4} \mathrm{COOH}$ & $\mathrm{CH}_{2} \mathrm{Cl}_{2}$ & 24 & 56 & 95 \\
6 & $3 \mathbf{N a O A c}$ & $\mathrm{CH}_{2} \mathrm{Cl}_{2}$ & 4 & 55 & 97 \\
\hline 7 & $3 \mathbf{a}$ & - & toluene & 24 & 42 & 97 \\
8 & $3 \mathbf{a}$ & - & acetone & 3 & 40 & 91 \\
$9^{d}$ & $3 \mathbf{3 a}$ & - & acetone & 2 & 37 & 87 \\
\hline
\end{tabular}

a. Unless indicated otherwise, the reactions were carried out on $0.10 \mathrm{mmol}$ at $25^{\circ} \mathrm{C}$ followed the general procedures for indicated time.

b. Isolated yield of $6 a$.

c. Determined by chiral HPLC using stationary phase chiral column.

d. With solvent acetone: $\mathrm{H}_{2} \mathrm{O}=4: 1$ (v:v).

When catalysts $\mathbf{3 b}$ and $\mathbf{3 c}$ with bulkier silyl groups were applied, slightly improvements of ee value were observed. Much longer reaction time was needed when relatively stronger acid $p-\mathrm{NO}_{2} \mathrm{C}_{6} \mathrm{H}_{4} \mathrm{COOH}$ was used as additive. And when acetone/water was used as the solvent system, the reaction proceeded rapidly, while a slightly decreased isolated yield and ee value were observed. 


\section{Control experiments and the identification of reaction intermediates}

A.

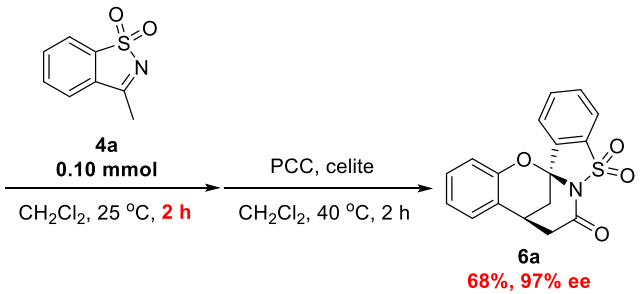

B.<smiles>O=CC=Cc1ccccc1O</smiles>

$1 a$
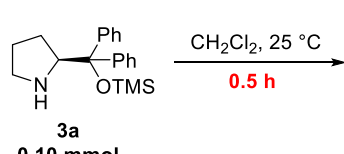

$0.10 \mathrm{mmol}$

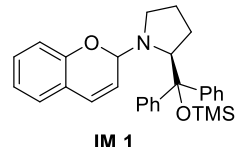

IM

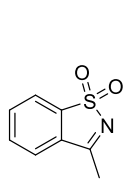

$4 a$

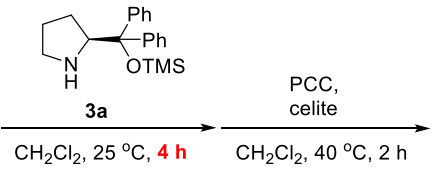

${ }_{2} \mathrm{Cl}_{2}, 40{ }^{\circ} \mathrm{C}, 2$

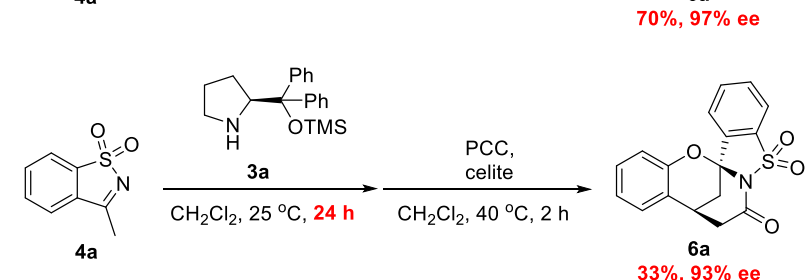

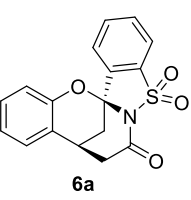

$6 \mathrm{a}$ $70 \%, 97 \%$ ee

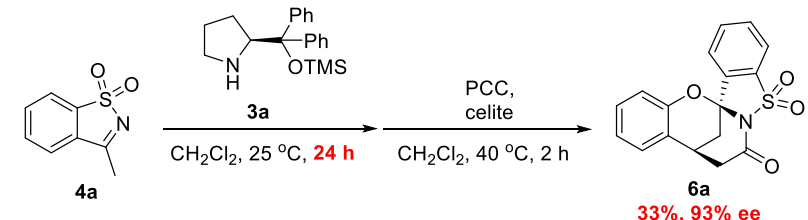

$1 a^{\prime}$

Reaction $A^{[1,4]}$.

1a (14.8 $\mathrm{mg}, 0.10 \mathrm{mmol})$ and $3 \mathrm{a}(32.5 \mathrm{mg}, 0.10 \mathrm{mmol})$ were pre-reacting in $1.0 \mathrm{~mL}$ dichloromethane at $25{ }^{\circ} \mathrm{C}$ for $0.5 \mathrm{~h}$. The intermediate 1 was purified with chromatography column (dichloromethane : methanol 10:1). Then dissolved it into $200 \mu \mathrm{L}$ dichloromethane, and $4 \mathrm{a}(18.1 \mathrm{mg}, 0.10 \mathrm{mmol})$ was added. The reaction was completed in $2 \mathrm{~h}$, then followed the general procedures to afford product $\mathbf{6 a}$.

\section{Reaction B.}

The reaction was conducted followed the general procedures.

\section{Reaction C.}

Substrate 1a' was synthesized by treating 1 a with $\mathrm{TsOH}$ (1.0 equiv) in $\mathrm{CHCl}_{3}$ at $40{ }^{\circ} \mathrm{C}$ for $48 \mathrm{~h}$. Then the reaction of 1a' was conducted followed the general procedures. However, 3a catalyzed the cascade reaction of $\mathbf{1} \mathbf{a}^{\prime}$ and $\mathbf{4 a}$ with much longer time (24 h), lower yield and ee value. 


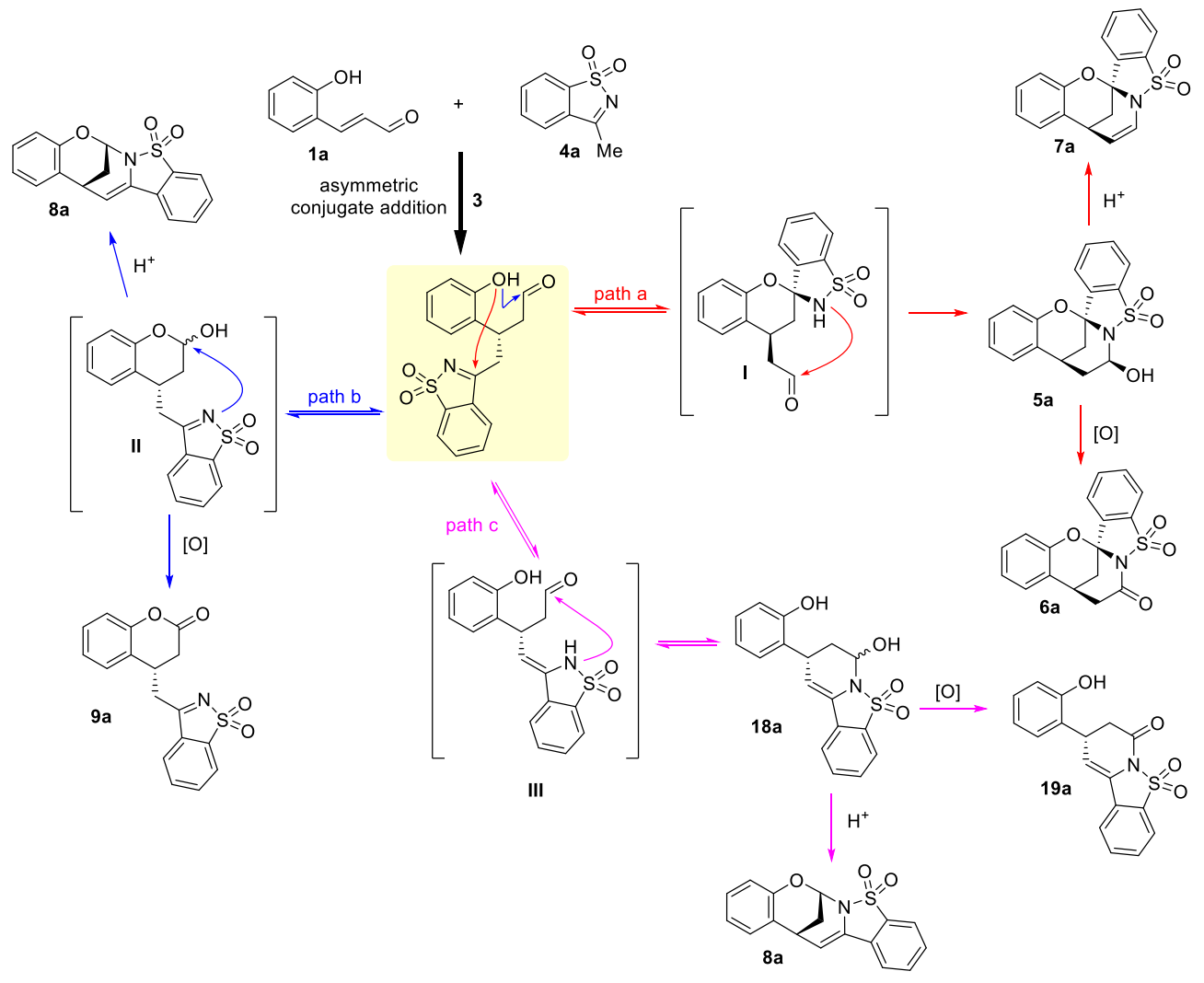

Indeed, except for path a and path $b$, it is proposed that there is another competitive reaction pathway, namely the ketimine tautomerized to the enamide followed by hemiaminal formation to give intermediate III (path c), followed by intramolecular cyclization to yield compound $18 \mathbf{a}$. The product $18 \mathrm{a}$ would be able to deliver bridged aminal 8a via iminium ion intermediates under acidic conditions or 2-piperidinone scaffold 19a under oxidation conditions. 
Accordingly, it is proposed that there are potentially three routes for the formation of compound $8 \mathbf{a}$.

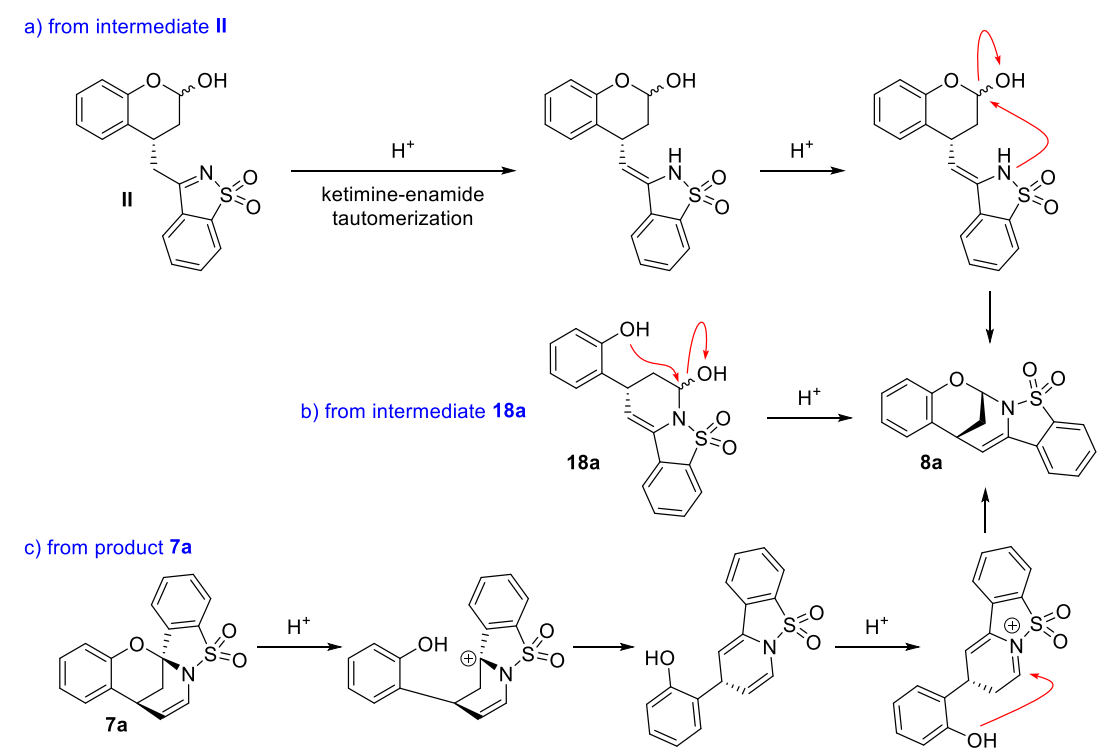

Unfortunately, since the reaction of $\mathbf{1 a}$ and $\mathbf{4 a}$ gave an inseparable mixture of isomers, we could not find more clear evidence for the formation of $\mathbf{8 a}$, namely from intermediate II or from intermediate $\mathbf{1 8 a}$, and what we can find was that compound 7a could transformed into 8 a under acidic conditions. 


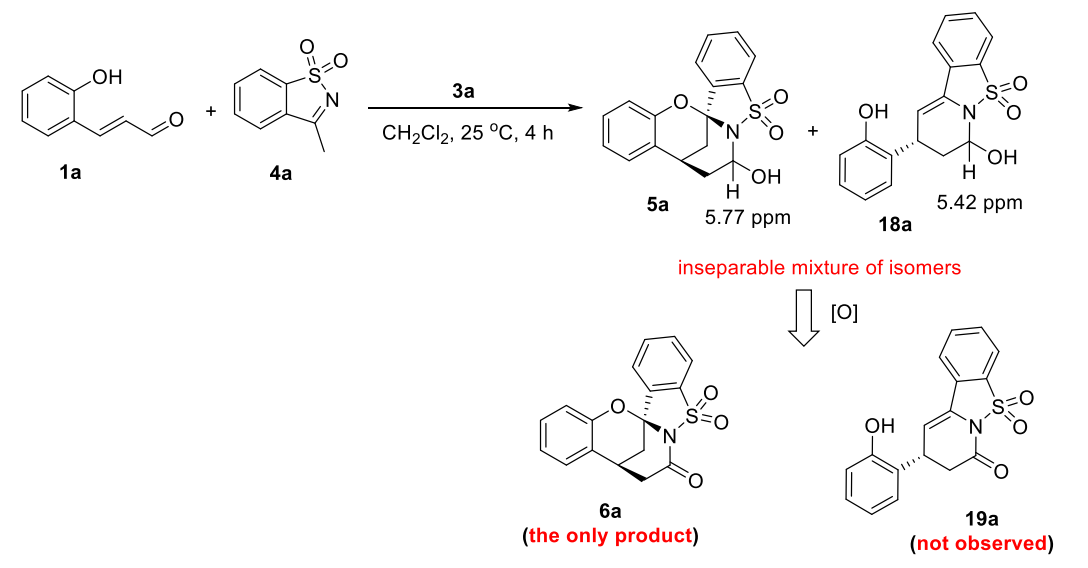

Next, we carried out the reaction of $\mathbf{1 a}$ and $\mathbf{4 a}$ under the optimized conditions, and products $\mathbf{5 a}$ and $\mathbf{1 8 a}$ were obtained as an inseparable mixture of isomers, whose structures were preliminary confirmed by NMR studies (Of course these results are not unequivocal, since the mixture of $5 \mathrm{a}$ and $18 \mathrm{a}$ are not pure and stable enough for a very clear NMR study). However, treatment of the mixture of $\mathbf{5 a}$ and $\mathbf{1 8 a}$ with PCC afforded $6 \mathbf{a}$ as the only product, while no product 19a was observed, which suggested that 18a was not stable under this oxidation conditions and might exist as an equilibrium with $\mathbf{5 a}$. 
Figure S1. ${ }^{1} \mathrm{H}$ NMR spectrum of $5 a$ and $18 a$

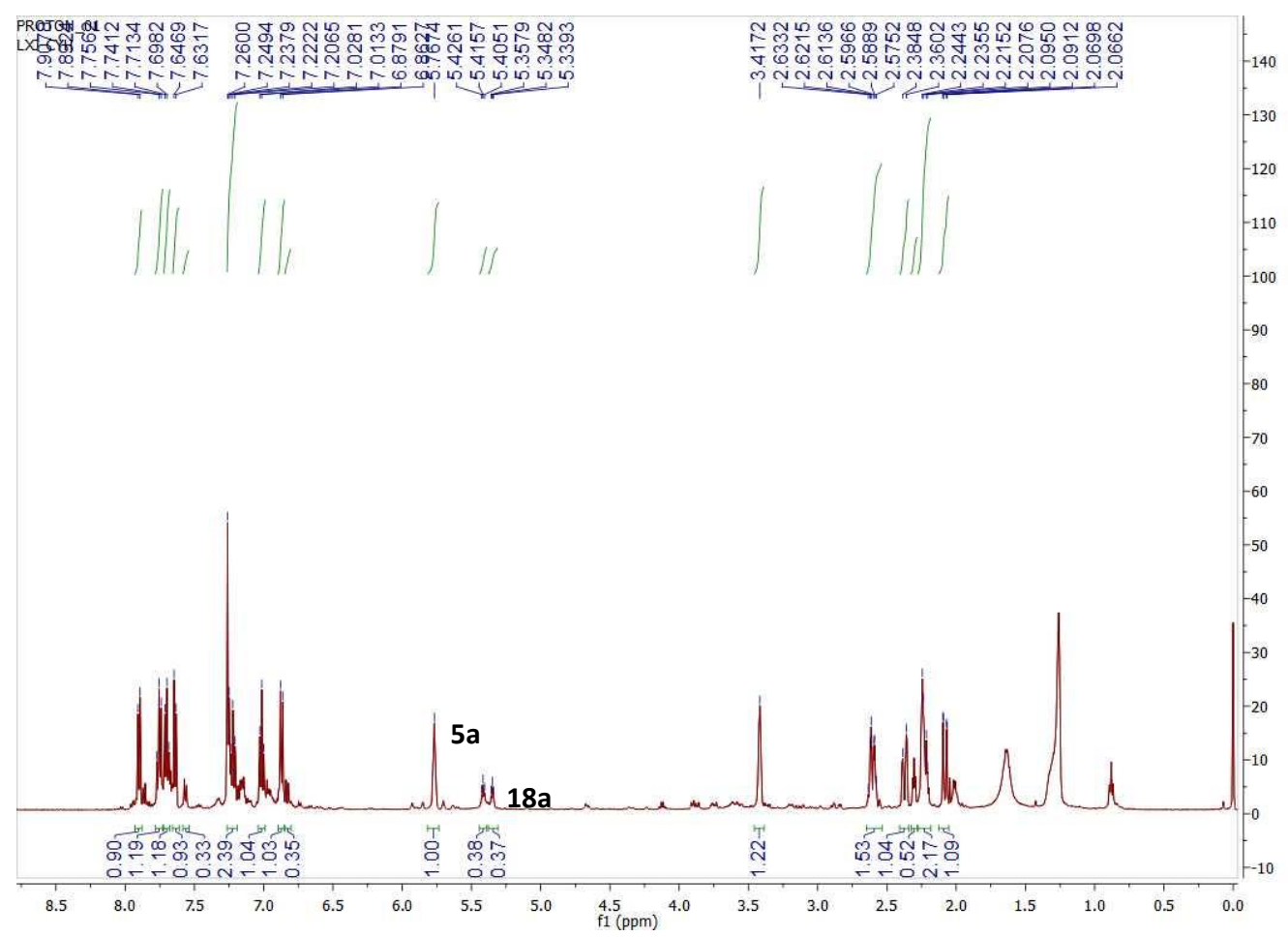

Figure S2. ${ }^{13} \mathrm{C}$ NMR spectrum of $\mathbf{5 a}$ and $\mathbf{1 8 a}$

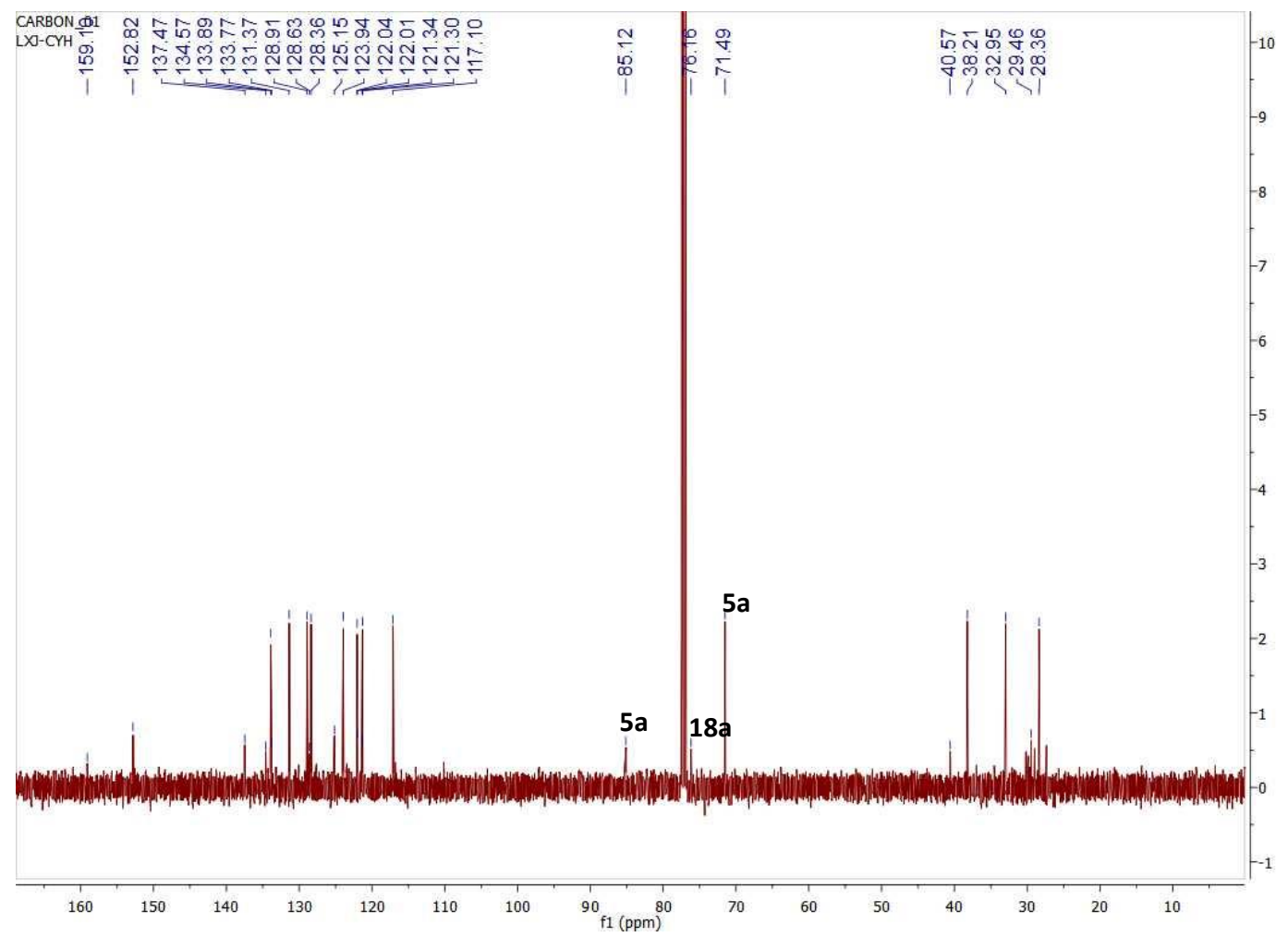




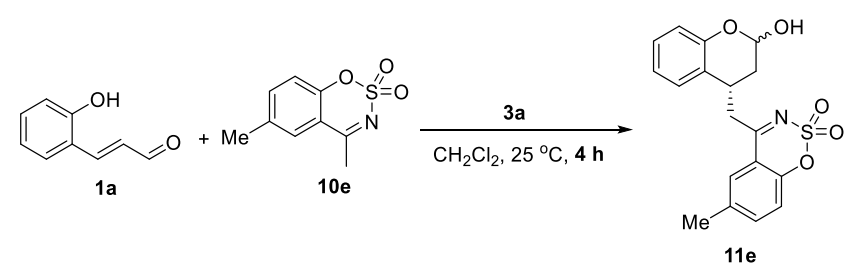

The reaction of $1 \mathrm{a}(0.11 \mathrm{mmol})$ and $10 \mathrm{e}(0.10 \mathrm{mmol})$ was conducted as the general procedure, after stirring at $25{ }^{\circ} \mathrm{C}$ for $4 \mathrm{~h}$, the reaction mixture was purified with chromatography column (petroleum ether : ethyl acetate 3:1). (the diastereoisomeric ratio of 11 e was about 5:4)

Figure S3. ${ }^{1} \mathrm{H}$ NMR spectrum of $11 \mathrm{e}$

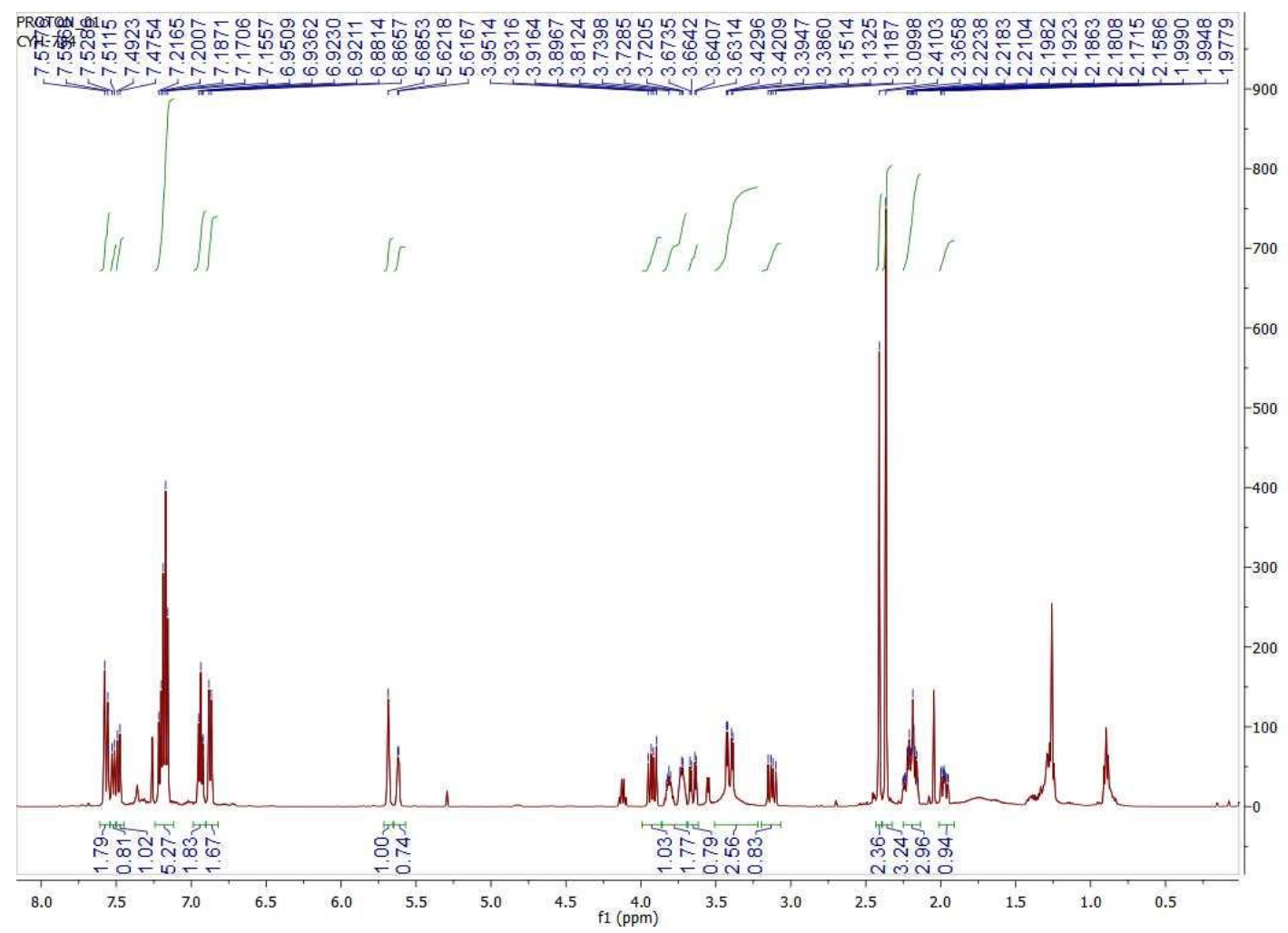


Figure S4. ${ }^{13} \mathrm{C}$ NMR spectrum of 11e

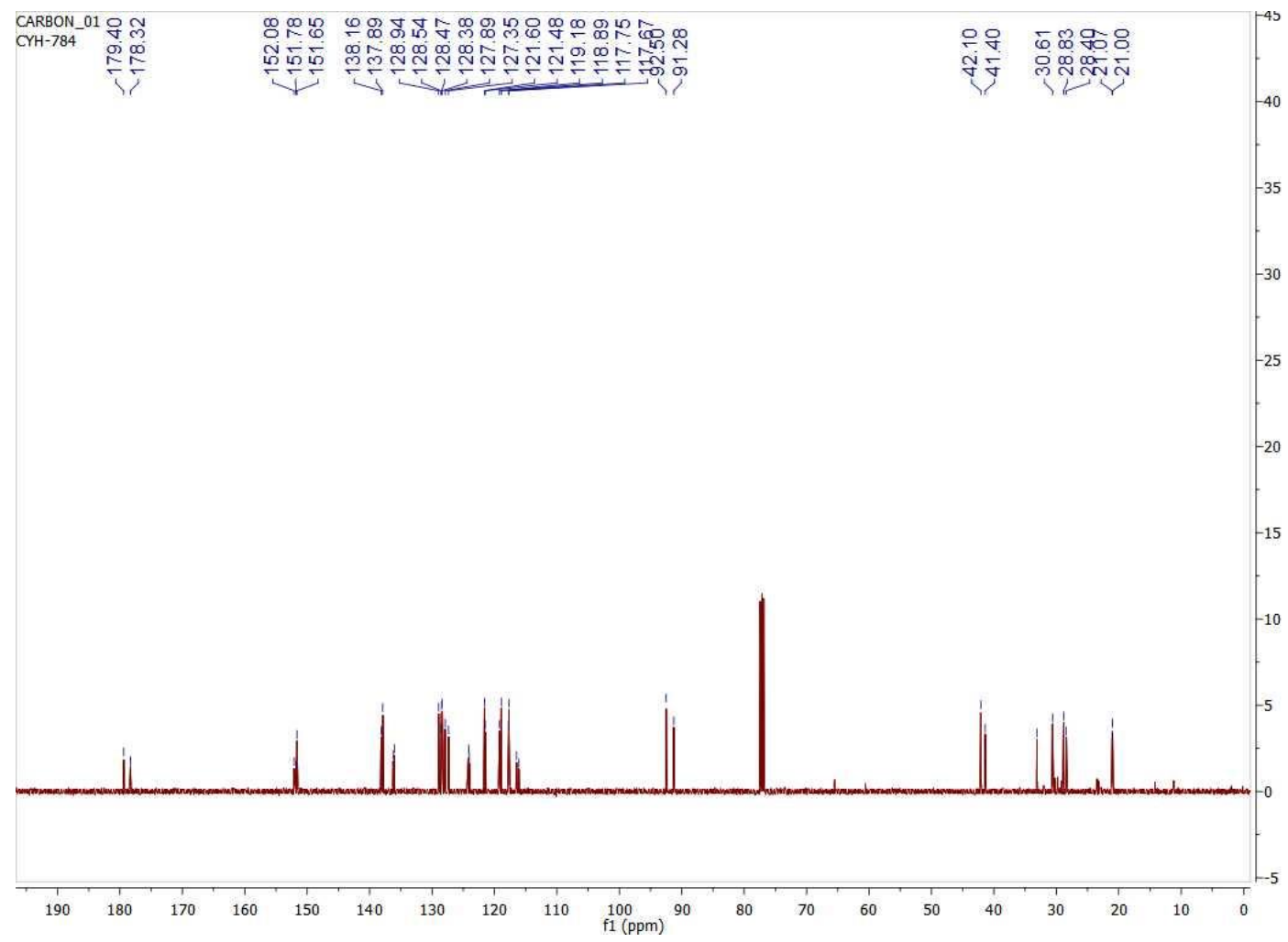




\section{Transformations of products}

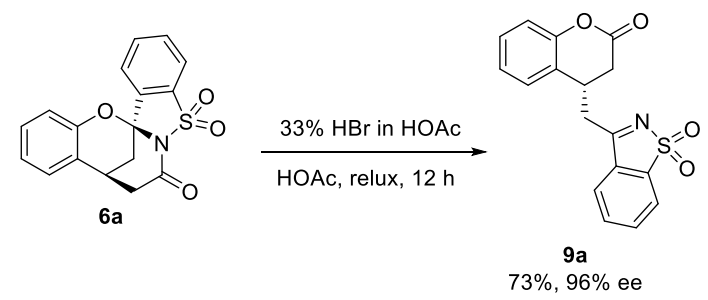

A $5 \mathrm{~mL}$ glass vial was charged with $6 \mathrm{a}(11 \mathrm{mg}, 0.03 \mathrm{mmol})$ in $500 \mu \mathrm{L} \mathrm{HOAc}$, then $50 \mu \mathrm{L}$ $33 \% \mathrm{HBr} / \mathrm{HOAc}$ solution was added. The reaction mixture was reflux for $12 \mathrm{~h}, 1 \mathrm{~mL}$ water was added to quench the reaction and the aqueous solution was extracted with dichloromethane $(3 \times 5 \mathrm{~mL})$. Collected the organic phase and reduced the solvent, the residue was purified with column chromatography (petroleum ether : ethyl acetate $3: 1$ ) to afford compound 9 a (8 mg, 73\%). ${ }^{1} \mathrm{H} \mathrm{NMR}\left(500 \mathrm{MHz}, \mathrm{CDCl}_{3}\right) \delta$ $7.91(\mathrm{~d}, J=7.4 \mathrm{~Hz}, 1 \mathrm{H}), 7.75(\mathrm{t}, J=7.5 \mathrm{~Hz}, 1 \mathrm{H}), 7.67(\mathrm{t}, J=7.6 \mathrm{~Hz}, 1 \mathrm{H}), 7.51(\mathrm{~d}, J=7.5$ $H z, 1 H), 7.35-7.26(m, 2 H), 7.10(t, J=6.9 \mathrm{~Hz}, 2 \mathrm{H}), 4.01-3.92(m, 1 \mathrm{H}), 3.29-3.16$ (m, 2H), $3.08-2.96(\mathrm{~m}, 2 \mathrm{H}) .{ }^{13} \mathrm{C}$ NMR $\left(125 \mathrm{MHz} \mathrm{CDCl}_{3}\right) \delta 173.4,167.5,151.5,139.7$, 134.2, 134.2, 131.0, 129.5, 128.4, 125.2, 124.8, 123.9, 122.8, 117.6, 35.7, 34.8, 32.2. $[\alpha]_{D}{ }^{20}-109.50\left(c=0.33\right.$ in $\left.\mathrm{CHCl}_{3}\right)$. ESI-HRMS: $[\mathrm{M}+\mathrm{H}]^{+}$calcd. For $\mathrm{C}_{17} \mathrm{H}_{14} \mathrm{NO}_{4} \mathrm{~S}^{+} \mathrm{m} / \mathrm{z}$ : 328.0638; found: 328.0641. The enantiomeric excess was determined by HPLC analysis on Daicel Chiralpak IC column ( $n$-hexane/i-PrOH/dichloromethane $=$ $40 / 10 / 50,1 \mathrm{~mL} / \mathrm{min}), \lambda=225 \mathrm{~nm}, t_{\text {major }}=12.83 \mathrm{~min}, t_{\text {minor }}=7.55 \mathrm{~min}$, ee $=96 \%$.

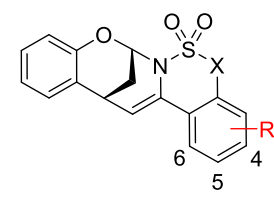

8a, $\mathrm{R}=\mathrm{H}, \mathrm{X}=$ none

13a, $R=H, X=O$

13b, $R=4-\mathrm{MeO}, X=O$

13c, $R=5-F, X=O$

13d, $R=5-\mathrm{Me}, X=0$

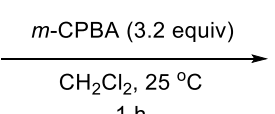

$1 \mathrm{~h}$

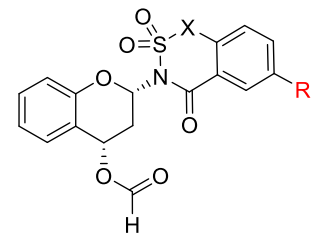

$14 \mathrm{a}^{\prime}, \mathrm{R}=\mathrm{H}, \mathrm{X}=$ none, $60 \%, 95 \%$ ee $14 a, R=H, X=O, 78 \%, 95 \%$ ee $14 c, R=F, X=0,75 \%, 91 \%$ ee

$14 d, R=M e, X=0,50 \%, 94 \%$ ee

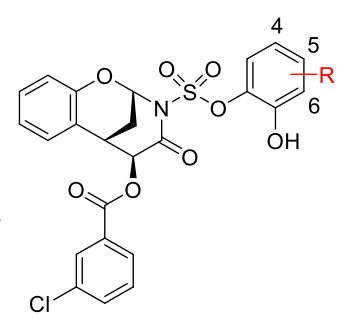

15b, $\mathrm{R}=4-\mathrm{MeO}, \mathrm{X}=\mathrm{O}, 79 \%, 99 \%$ ee 15d, $R=5-M e, X=0,25 \%, 93 \%$ ee $15 e, R=6-\mathrm{MeO}, X=0,61 \%, 99 \%$ ee

\section{General procedure}

Compounds 8a or 13 ( $0.10 \mathrm{mmol}, 1.0$ equiv) was dissolved in $1 \mathrm{~mL}$ dichloromethane, and $\mathrm{m}$-CPBA (85\%, $65 \mathrm{mg}, 3.2$ equiv) was added in portions. And the colorless 
solution was turned into white suspension rapidly. The reaction was completed in less than $1 \mathrm{~h}$, then another $2 \mathrm{~mL}$ dichloromethane was added. The colorless solution was washed with $\mathrm{Na}_{2} \mathrm{~S}_{2} \mathrm{O}_{3}$ aqueous solution and $\mathrm{NaHCO}_{3}$ aqueous solution sequentially, and then the organic phase was concentrated and the residue was purified with chromatography column (petroleum ether : ethyl acetate : dichloromethane 10:1:1).

Scheme S1. The possible mechanism about the $m$-CPBA oxidation of 8 a and 13:

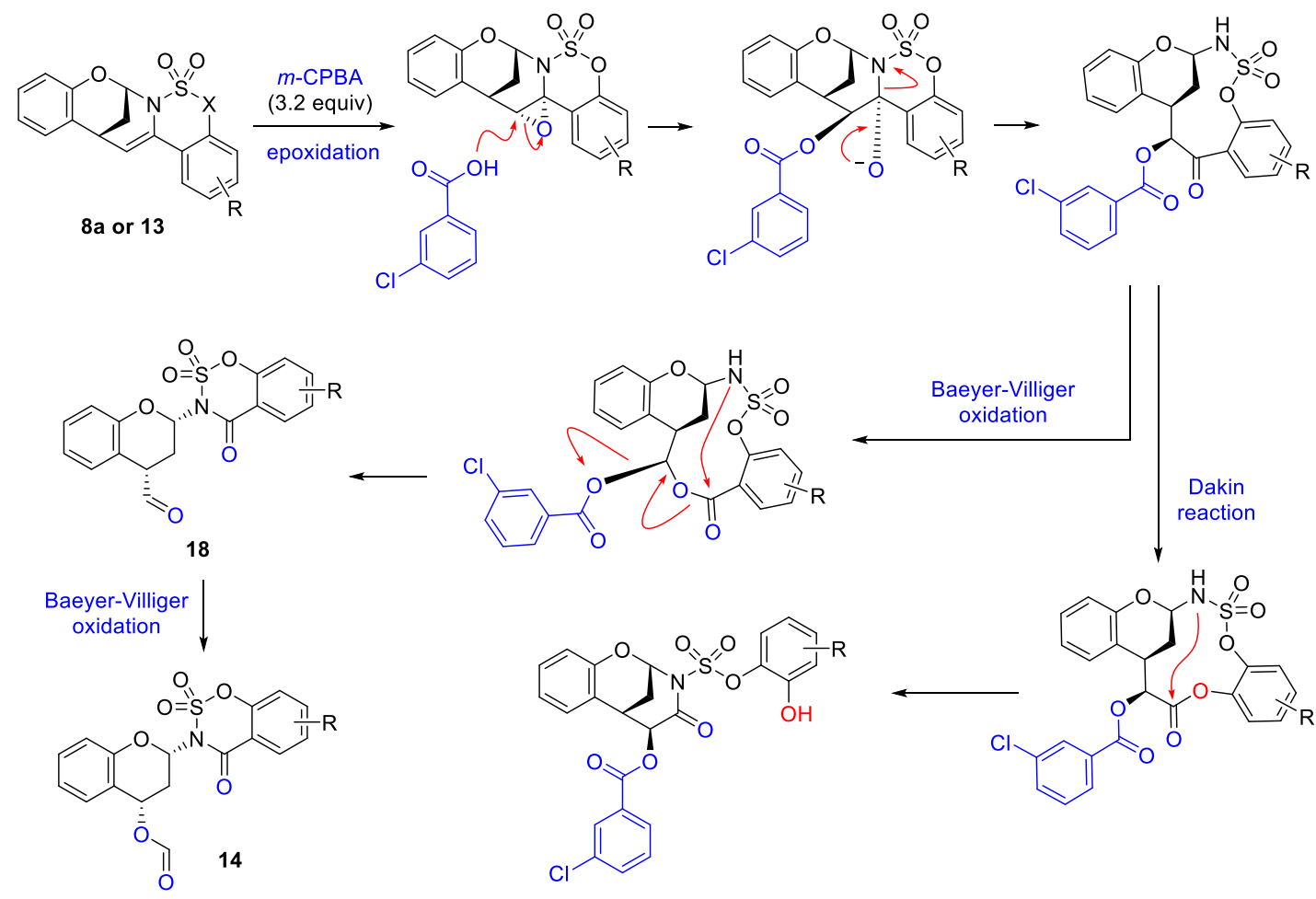

By treatment with $m$-CPBA oxidation, the enamine moiety in 13a could undergo an epoxidation followed by the ring cleavage, and then a Baeyer-Villiger type oxidation occurred to give the aldehyde intermediate 18 . The 18 will be rapidly further oxidized into 14a. And we found it is observed that the electronic effects of the substituents on the aromatic ring of $\mathrm{N}$-sulfonyl ketimine play a critical role in this cascade transformation. An electron-withdrawing group, such as the fluorine atom providing $14 \mathrm{c}$ as the only product, while on introducing a methyl group instead of fluorine atom, the normal aminal product $14 \mathrm{~d}$ accompanied by unexpected bridged aminal 15d was obtained in a 2:1 ratio (based on the isolated yield). We proposed 
that a Dakin type rearrangement after epoxidation because of the electron-donating methyl group. As expected, the stronger electron-donating effect of the methoxy group on the ortho and para positions resulted in the formation of the bridged aminal $\mathbf{1 5 b}$ and $\mathbf{1 5 e}$, respectively, as the sole product in $79 \%$ and $61 \%$ yields.

\section{The characterization of 14 and 15}

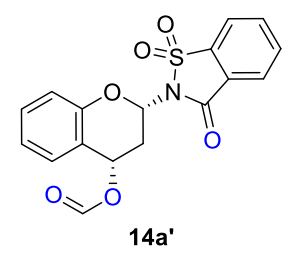

$14 a^{\prime}$ was synthesized following the general method ( $22 \mathrm{mg}, 60 \%$ yield) as a white solid. ${ }^{1} \mathrm{H}$ NMR (500 MHz, $\left.\mathrm{CDCl}_{3}\right) \delta 8.28(\mathrm{~d}, J=0.6 \mathrm{~Hz}, 1 \mathrm{H}), 8.12$ (d, J = 7.5 Hz, 1H), 7.95 $-7.85(m, 2 H), 7.90-7.84(m, 1 H), 7.26-7.21(m, 2 H), 7.02(t, J=7.3 \mathrm{~Hz}, 1 \mathrm{H}), 6.95-$ $6.89(\mathrm{~m}, 1 \mathrm{H}), 6.38(\mathrm{dd}, J=10.1,6.6 \mathrm{~Hz}, 1 \mathrm{H}), 6.24(\mathrm{dd}, J=12.1,2.0 \mathrm{~Hz}, 1 \mathrm{H}), 3.13-3.01$ (m, 1H), 2.95 (ddd, $J=12.6,6.5,2.1 \mathrm{~Hz}, 1 \mathrm{H}) .{ }^{13} \mathrm{C} \mathrm{NMR}\left(125 \mathrm{MHz}, \mathrm{CDCl}_{3}\right) \delta$ 160.9, $158.5,153.2,138.1,135.7,134.8,130.3,127.1,126.5,126.0,122.2,121.2,120.1$, 117.6, 77.6, 66.7, 30.5. $[\alpha]_{D}^{20}+99.09\left(c=2.08\right.$ in $\left.\mathrm{CHCl}_{3}\right)$. ESI-HRMS: $[\mathrm{M}+\mathrm{H}]^{+} c a l c d$. For $\mathrm{C}_{17} \mathrm{H}_{14} \mathrm{NO}_{6} \mathrm{~S}^{+} \mathrm{m} / \mathrm{z}$ : 360.0536; found: 360.0533. The enantiomeric excess was determined by HPLC analysis on Daicel Chiralpak IB column ( $n$-hexane $/$ i-PrOH/dichloromethane $=70 / 27 / 3,1 \mathrm{~mL} / \mathrm{min}$ ), $\lambda=216 \mathrm{~nm}, t_{\text {major }}=10.59$ $\min , t_{\text {minor }}=9.91 \mathrm{~min}$, ee $=95 \%$.

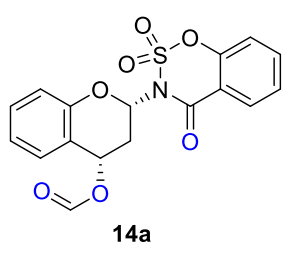

14a was synthesized following the general method ( $29 \mathrm{mg}, 77 \%$ yield) as a white solid. ${ }^{1} \mathrm{H}$ NMR $\left(500 \mathrm{MHz}, \mathrm{CDCl}_{3}\right) \delta 8.27(\mathrm{~s}, 1 \mathrm{H}), 8.19(\mathrm{~d}, J=7.8 \mathrm{~Hz}, 1 \mathrm{H}), 7.81-7.72(\mathrm{~m}$, 1H), $7.49(t, J=7.6 \mathrm{~Hz}, 1 \mathrm{H}), 7.33(\mathrm{~d}, J=8.3 \mathrm{~Hz}, 1 \mathrm{H}), 7.28-7.23(\mathrm{~m}, 2 \mathrm{H}), 7.03(\mathrm{t}, J=7.5$ $\mathrm{Hz}, 1 \mathrm{H}), 6.95(\mathrm{~d}, J=8.2 \mathrm{~Hz}, 1 \mathrm{H}), 6.69(\mathrm{dd}, J=12.1,2.0 \mathrm{~Hz}, 1 \mathrm{H}), 6.34(\mathrm{dd}, J=10.5,6.3$ $\mathrm{Hz}, 1 \mathrm{H}), 3.08(\mathrm{dd}, J=23.2,12.1 \mathrm{~Hz}, 1 \mathrm{H}), 2.77(\mathrm{ddd}, J=12.3,6.2,2.1 \mathrm{~Hz}, 1 \mathrm{H}) .{ }^{13} \mathrm{C} \mathrm{NMR}$ $\left(125 \mathrm{MHz} \mathrm{CDCl}_{3}\right) \delta 160.9,160.4,153.1,150.8,137.0,131.1,130.2,127.4,126.8$, 
122.3, 120.3, 118.7, 117.5, 117.3, 79.1, 66.9, 31.5. $[\alpha]_{D}^{20}+61.08\left(c=1.17\right.$ in $\left.\mathrm{CHCl}_{3}\right)$. ESI-HRMS: $[\mathrm{M}+\mathrm{H}]^{+}$calcd. For $\mathrm{C}_{17} \mathrm{H}_{14} \mathrm{NO}_{7} \mathrm{~S}^{+} \mathrm{m} / \mathrm{z}$ : 376.0485; found: 376.0481 . The enantiomeric excess was determined by HPLC analysis on Daicel Chiralpak IB column ( $n$-hexane $/ i-\mathrm{PrOH}=70 / 30,1 \mathrm{~mL} / \mathrm{min}$ ), $\lambda=210 \mathrm{~nm}, t_{\text {major }}=8.63 \mathrm{~min}, t_{\text {minor }}=7.45 \mathrm{~min}$, ee $=95 \%$.

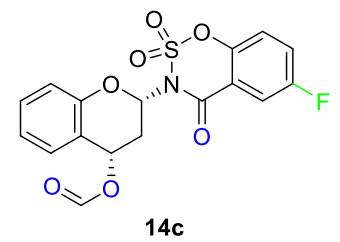

14c was synthesized following the general method (30 $\mathrm{mg}, 76 \%$ yield) as a white solid. ${ }^{1} \mathrm{H}$ NMR $\left(500 \mathrm{MHz}, \mathrm{CDCl}_{3}\right) \delta 8.30-8.24(\mathrm{~m}, 1 \mathrm{H}), 7.85(\mathrm{dd}, J=7.6,3.1 \mathrm{~Hz}, 1 \mathrm{H})$, 7.45 (ddd, $J=9.1,7.5,3.1 \mathrm{~Hz}, 1 \mathrm{H}), 7.33(\mathrm{dd}, J=9.0,4.0 \mathrm{~Hz}, 1 \mathrm{H}), 7.28-7.23(\mathrm{~m}, 2 \mathrm{H})$, $7.03(\mathrm{t}, J=7.3 \mathrm{~Hz}, 1 \mathrm{H}), 6.94(\mathrm{~d}, J=8.1 \mathrm{~Hz}, 1 \mathrm{H}), 6.67(\mathrm{dd}, J=12.2,2.3 \mathrm{~Hz}, 1 \mathrm{H}), 6.33$ (dd, $J=10.5,6.3 \mathrm{~Hz}, 1 \mathrm{H}$ ), 3.05 (dd, $J=23.1,12.2 \mathrm{~Hz}, 1 \mathrm{H}$ ), 2.76 (ddd, $J=12.4,6.3,2.4 \mathrm{~Hz}$, 1H). ${ }^{13} \mathrm{C}$ NMR $\left(125 \mathrm{MHz}, \mathrm{CDCl}_{3}\right) \delta 161.5,160.8,159.5$ (d, J = 2.4 Hz), 159.5, 153.0, 146.7 (d, $J=2.6 \mathrm{~Hz}$ ), 130.3, 126.8, 124.2 (d, $J=24.5 \mathrm{~Hz}$ ), 122.4, 120.7 (d, $J=8.1 \mathrm{~Hz}$ ), $120.3,118.8(\mathrm{~d}, J=8.3 \mathrm{~Hz}), 117.5,117.2(\mathrm{~d}, J=25.9 \mathrm{~Hz}), 79.3,66.7,31.4 .[\alpha]_{D}{ }^{20}$ +77.58 ( $c=2.00$ in $\mathrm{CHCl}_{3}$ ). ESI-HRMS: $[\mathrm{M}+\mathrm{H}]^{+}$calcd. For $\mathrm{C}_{17} \mathrm{H}_{13} \mathrm{FNO}_{7} \mathrm{~S}^{+} \mathrm{m} / \mathrm{z}$ : 394.0391; found: 394.0392. The enantiomeric excess was determined by HPLC analysis on Daicel Chiralpak IB column ( $n$-hexane/i-PrOH $=75 / 25,1 \mathrm{~mL} / \mathrm{min}$ ), $\lambda=210 \mathrm{~nm}, t_{\text {major }}=$ $10.21 \mathrm{~min}, t_{\text {minor }}=8.81 \mathrm{~min}$, ee $=91 \%$.

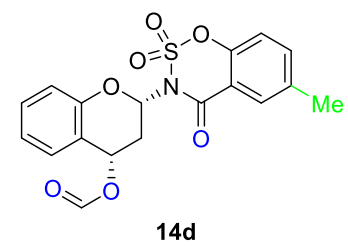

14d was synthesized following the general method (20 mg, 51\% yield) as a white solid. ${ }^{1} \mathrm{H}$ NMR (500 MHz, $\left.\mathrm{CDCl}_{3}\right) \delta 8.27$ (d, J=0.7 Hz, $1 \mathrm{H}$ ), 7.96 (d, J=1.0 Hz, 1H), 7.53 (dd, $J=8.4,1.6 \mathrm{~Hz}, 1 \mathrm{H}), 7.27-7.18(\mathrm{~m}, 2 \mathrm{H}), 7.20$ (d, $J=8.4 \mathrm{~Hz}, 1 \mathrm{H}), 7.02(\mathrm{dd}, J=11.1$, $3.9 \mathrm{~Hz}, 1 \mathrm{H}), 6.94$ (d, J = 8.1 Hz, 1H), 6.68 (dd, J = 12.2, 2.2 Hz, 1H), 6.33 (dd, J = 10.6, $6.4 \mathrm{~Hz}, 1 \mathrm{H}$ ), 3.07 (dd, $J=23.1,12.2 \mathrm{~Hz}, 1 \mathrm{H}$ ), 2.76 (ddd, $J=12.4,6.3,2.3 \mathrm{~Hz}, 1 \mathrm{H}$ ), 2.45 (s, 3H). ${ }^{13} \mathrm{C} \mathrm{NMR}\left(125 \mathrm{MHz}, \mathrm{CDCl}_{3}\right) \delta 160.9,160.6,153.2,148.8,137.7,131.0,130.2$, 
$126.8,122.2,120.3,118.4,117.5,116.9,79.0,66.9,31.6,21.0 .[\alpha]_{D}^{20}+69.52(c=2.17$ in $\mathrm{CHCl}_{3}$ ). ESI-HRMS: $[\mathrm{M}+\mathrm{H}]^{+}$calcd. For $\mathrm{C}_{18} \mathrm{H}_{16} \mathrm{NO}_{7} \mathrm{~S}^{+} \mathrm{m} / \mathrm{z}$ : 390.0642 ; found: 390.0645 . The enantiomeric excess was determined by HPLC analysis on Daicel Chiralpak IB column ( $n$-hexane $/ i-\mathrm{PrOH}=75 / 25,1 \mathrm{~mL} / \mathrm{min}), \lambda=210 \mathrm{~nm}, t_{\text {major }}=8.89 \mathrm{~min}, t_{\text {minor }}=$ $8.05 \min$, ee $=94 \%$.

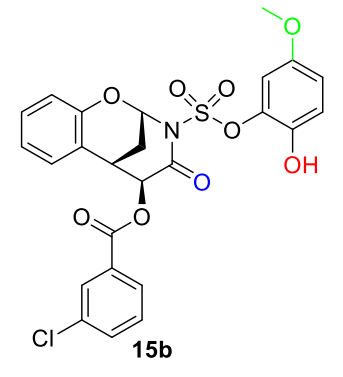

15b was synthesized following the general method ( $43 \mathrm{mg}, 79 \%$ yield) as a white solid. ${ }^{1} \mathrm{H}$ NMR $\left(500 \mathrm{MHz}, \mathrm{CDCl}_{3}\right) \delta 8.05(\mathrm{~s}, 1 \mathrm{H}), 7.98(\mathrm{~d}, J=7.8 \mathrm{~Hz}, 1 \mathrm{H}), 7.62(\mathrm{~d}, J=8.0$ $\mathrm{Hz}, 1 \mathrm{H}), 7.45$ (t, J= $7.9 \mathrm{~Hz}, 1 \mathrm{H}), 7.36$ (d, J = 7.0 Hz, 1H), 7.29 (dd, J=11.3, 4.2 Hz, 1H), $7.10(\mathrm{t}, J=7.2 \mathrm{~Hz}, 1 \mathrm{H}), 6.99-6.86(\mathrm{~m}, 3 \mathrm{H}), 6.82(\mathrm{dd}, J=9.0,2.8 \mathrm{~Hz}, 1 \mathrm{H}), 6.69(\mathrm{~s}, 1 \mathrm{H})$, $6.42(\mathrm{~d}, J=1.1 \mathrm{~Hz}, 1 \mathrm{H}), 5.70(\mathrm{~s}, 1 \mathrm{H}), 3.77(\mathrm{~s}, 3 \mathrm{H}), 3.48(\mathrm{~s}, 1 \mathrm{H}), 2.64(\mathrm{~d}, J=14.0 \mathrm{~Hz}, 1 \mathrm{H})$, $2.39(\mathrm{~d}, J=14.0 \mathrm{~Hz}, 1 \mathrm{H}) .{ }^{13} \mathrm{C} \operatorname{NMR}\left(125 \mathrm{MHz}, \mathrm{CDCl}_{3}\right) \delta 166.4,163.9,153.2,149.7$, $142.7,135.9,135.1,134.3,131.0,130.4,130.2$, 130.2, 128.5, 123.6, 118.8, 118.4, 118.2, 115.8, 108.0, 82.1, 74.6, 56.2, 32.8, 23.2. $[\alpha]_{D}{ }^{20}+26.95\left(c=1.33\right.$ in $\left.\mathrm{CHCl}_{3}\right)$. ESI-HRMS: $[\mathrm{M}+\mathrm{H}]^{+}$calcd. For $\mathrm{C}_{25} \mathrm{H}_{21} \mathrm{CINO}_{9} \mathrm{~S}^{+} \mathrm{m} / \mathrm{z}$ : 546.0620; found: 546.0618. The enantiomeric excess was determined by HPLC analysis on Daicel Chiralpak IC column ( $n$-hexane $/ i-\mathrm{PrOH} /$ dichloromethane $=80 / 17 / 3,1 \mathrm{~mL} / \mathrm{min}), \lambda=225 \mathrm{~nm}, t_{\text {major }}=13.26$ $\min , t_{\text {minor }}=14.15 \mathrm{~min}$, ee $=99 \%$.

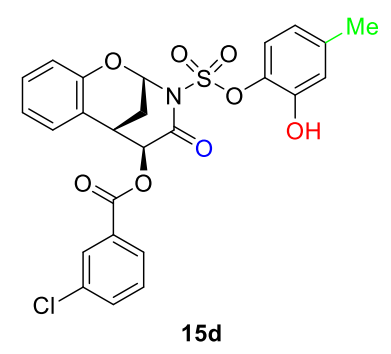

$15 d$ was synthesized following the general method (13 $\mathrm{mg}, 25 \%$ yield) as a white solid. ${ }^{1} \mathrm{H}$ NMR $\left(500 \mathrm{MHz}, \mathrm{CDCl}_{3}\right) \delta 8.06(\mathrm{~s}, 1 \mathrm{H}), 7.98(\mathrm{~d}, J=7.8 \mathrm{~Hz}, 1 \mathrm{H}), 7.62(\mathrm{~d}, J=7.9$ $\mathrm{Hz}, 1 \mathrm{H}), 7.45(\mathrm{t}, J=7.9 \mathrm{~Hz}, 1 \mathrm{H}), 7.36(\mathrm{~d}, J=7.5 \mathrm{~Hz}, 1 \mathrm{H}), 7.32-7.22(\mathrm{~m}, 2 \mathrm{H}), 7.10(\mathrm{t}, J=$ 
$7.4 \mathrm{~Hz}, 1 \mathrm{H}), 7.02(\mathrm{~s}, 1 \mathrm{H}), 6.90(\mathrm{~d}, J=8.2 \mathrm{~Hz}, 1 \mathrm{H}), 6.82(\mathrm{~s}, 1 \mathrm{H}), 6.70(\mathrm{~d}, J=7.4 \mathrm{~Hz}, 1 \mathrm{H})$, $6.40(\mathrm{~s}, 1 \mathrm{H}), 5.70(\mathrm{~s}, 1 \mathrm{H}), 3.48(\mathrm{~s}, 1 \mathrm{H}), 2.63(\mathrm{~d}, J=14.0 \mathrm{~Hz}, 1 \mathrm{H}), 2.38$ (d, J = $14.2 \mathrm{~Hz}$, $1 \mathrm{H}), 2.31(\mathrm{~s}, 3 \mathrm{H}) .{ }^{13} \mathrm{C} N M R\left(125 \mathrm{MHz}, \mathrm{CDCl}_{3}\right) \delta 166.4,163.9,149.8,148.5,140.0,135.1$, $134.3,134.1,130.9,130.4,130.2,130.2,128.5,123.6,122.4,121.3,119.0,118.4$, 118.2, 82.0, 74.7, 32.8, 23.2, 21.4. $[\alpha]_{D}^{20}+26.28\left(c=0.50\right.$ in $\left.\mathrm{CHCl}_{3}\right)$. ESI-HRMS: $[\mathrm{M}+\mathrm{H}]^{+}$ calcd. For $\mathrm{C}_{25} \mathrm{H}_{21} \mathrm{CINO}_{8} \mathrm{~S}^{+} \mathrm{m} / \mathrm{z}$ : 530.0671; found: 530.0676 . The enantiomeric excess was determined by HPLC analysis on Daicel Chiralpak IA column ( $n$-hexane/i-PrOH $=$ $60 / 40,1 \mathrm{~mL} / \mathrm{min}), \lambda=210 \mathrm{~nm}, t_{\text {major }}=37.32 \mathrm{~min}, t_{\text {minor }}=9.59 \mathrm{~min}$, ee $=93 \%$.

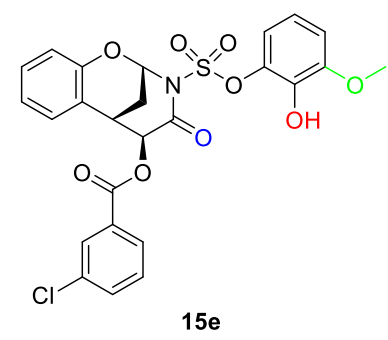

15e was synthesized following the general method (33 $\mathrm{mg}, 60 \%$ yield) as a white solid. ${ }^{1} \mathrm{H}$ NMR $\left(500 \mathrm{MHz}, \mathrm{CDCl}_{3}\right) \delta 8.04(\mathrm{~s}, 1 \mathrm{H}), 7.98(\mathrm{~d}, J=7.9 \mathrm{~Hz}, 1 \mathrm{H}), 7.60(\mathrm{dd}, J=$ 8.0, 1.0 Hz, 1H), 7.44 (t, J = 7.9 Hz, 1H), 7.35 (d, J = 7.6 Hz, 1H), 7.24 (dd, J = 12.7, 5.5 $\mathrm{Hz}, 1 \mathrm{H}), 7.08(\mathrm{t}, J=7.5 \mathrm{~Hz}, 1 \mathrm{H}), 6.96(\mathrm{dd}, J=7.8,1.9 \mathrm{~Hz}, 1 \mathrm{H}), 6.89-6.80(\mathrm{~m}, 2 \mathrm{H}), 6.76$ (d, $J=8.2 \mathrm{~Hz}, 1 \mathrm{H}$ ), 6.39 (d, J=1.0 Hz, 1H), $6.30(\mathrm{~s}, 1 \mathrm{H}), 5.69(\mathrm{~s}, 1 \mathrm{H}), 3.86(\mathrm{~s}, 3 \mathrm{H}), 3.47$ (s, 1H), $2.66(\mathrm{~d}, J=14.0 \mathrm{~Hz}, 1 \mathrm{H}), 2.35(\mathrm{~d}, J=14.0 \mathrm{~Hz}, 1 \mathrm{H}) .{ }^{13} \mathrm{C} \mathrm{NMR}\left(125 \mathrm{MHz}, \mathrm{CDCl}_{3}\right) \delta$ $165.1,164.0,149.9,148.9,138.9,136.6,135.0,134.2,130.6,130.2,130.1,130.1$, $128.4,123.2,119.4,118.6,118.1,115.5,110.9,81.7,74.7,56.5,33.0,23.4 .[\alpha]_{D}^{20}$ $+36.63\left(c=0.33\right.$ in $\mathrm{CHCl}_{3}$ ). ESI-HRMS: $[\mathrm{M}+\mathrm{H}]^{+}$calcd. For $\mathrm{C}_{25} \mathrm{H}_{21} \mathrm{CINO}_{9} \mathrm{~S}^{+} \mathrm{m} / \mathrm{z}$ : 546.0620; found: 546.0616. The enantiomeric excess was determined by HPLC analysis on Daicel Chiralpak IC column ( $n$-hexane/i-PrOH $=60 / 40,1 \mathrm{~mL} / \mathrm{min}$ ), $\lambda=210 \mathrm{~nm}, t_{\text {major }}=$ $25.60 \mathrm{~min}, t_{\text {minor }}=36.54 \mathrm{~min}$, ee $=99 \%$.

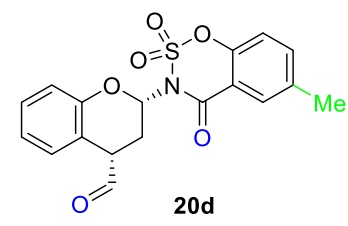

20d (21 mg, 56\%) with a few 14d and $15 d .{ }^{1} \mathrm{H}$ NMR $\left(500 \mathrm{MHz}, \mathrm{CDCl}_{3}\right) \delta 9.87(\mathrm{~s}, 1 \mathrm{H})$, $7.96(\mathrm{~s}, 1 \mathrm{H}), 7.53(\mathrm{~d}, J=7.1 \mathrm{~Hz}, 1 \mathrm{H}), 7.34(\mathrm{~d}, J=7.5 \mathrm{~Hz}, 1 \mathrm{H}), 7.27(\mathrm{dd}, J=9.3,5.7 \mathrm{~Hz}$, 
1H), $7.20(\mathrm{~d}, J=8.3 \mathrm{~Hz}, 1 \mathrm{H}), 7.07(\mathrm{t}, J=7.6 \mathrm{~Hz}, 1 \mathrm{H}), 7.03-6.96(\mathrm{~m}, 1 \mathrm{H}), 6.43(\mathrm{dd}, J=$ 11.7, $2.7 \mathrm{~Hz}, 1 \mathrm{H}), 3.83(\mathrm{~d}, J=5.6 \mathrm{~Hz}, 1 \mathrm{H}), 2.98(\mathrm{td}, J=12.4,5.9 \mathrm{~Hz}, 1 \mathrm{H}), 2.81(\mathrm{~d}, J=$ $13.2 \mathrm{~Hz}, 1 \mathrm{H}), 2.45$ (s, 3H). ${ }^{13} \mathrm{C}$ NMR (125 MHz, $\left.\mathrm{CDCl}_{3}\right) \delta 198.9,160.5,154.0,148.8$, 137.6, 137.6, 130.9, 129.6, 129.6, 122.4, 118.3, 118.3, 117.1, 115.2, 78.9, 48.4, 24.1, 21.0. ESI-HRMS: $[\mathrm{M}+\mathrm{H}]^{+}$calcd. For $\mathrm{C}_{18} \mathrm{H}_{16} \mathrm{NO}_{6} \mathrm{~S}^{+} \mathrm{m} / \mathrm{z}$ : 374.0693; found: 374.0695 .

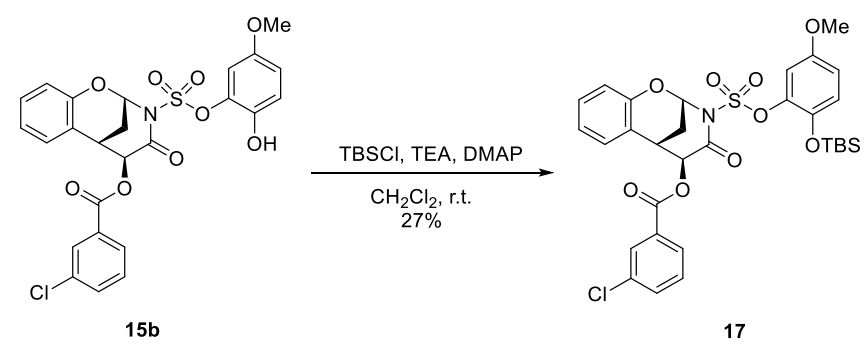

A glass vial was charged with 15b (24 mg, $0.04 \mathrm{mmol}, 1.0$ equiv), triethylamine (TEA, $8 \mu \mathrm{L}, 0.06 \mathrm{mmol}, 1.5$ equiv) and $\mathrm{N}, \mathrm{N}$-dimethyl-4-aminopyridine (DMAP, $0.5 \mathrm{mg}, 10$ $\mathrm{mol} \%$ ) in $500 \mu \mathrm{L}$ dichloromethane. Then tert-butyldimethylsilyl chloride (8 mg, 0.05 mmol, 1.2 equiv) was added, the reaction mixture was stirred at ambient temperature for $12 \mathrm{~h}$. The product 17 (7 mg, 27\%) was purified with chromatography column (petroleum ether : ethyl acetate 15:1) as a white solid for growing single crystals to identify the absolute configuration of $15 \mathrm{~b} .{ }^{1} \mathrm{H} \mathrm{NMR}\left(500 \mathrm{MHz}, \mathrm{CDCl}_{3}\right) \delta$ $8.06(\mathrm{~s}, 1 \mathrm{H}), 8.01(\mathrm{~d}, J=7.8 \mathrm{~Hz}, 1 \mathrm{H}), 7.64-7.57(\mathrm{~m}, 1 \mathrm{H}), 7.44(\mathrm{t}, J=7.9 \mathrm{~Hz}, 1 \mathrm{H}), 7.36$ (d, $J=7.5 \mathrm{~Hz}, 1 \mathrm{H}), 7.31-7.26(\mathrm{~m}, 1 \mathrm{H}), 7.08(\mathrm{t}, J=7.5 \mathrm{~Hz}, 1 \mathrm{H}), 6.88(\mathrm{~d}, J=8.5 \mathrm{~Hz}, 1 \mathrm{H})$, $6.84(\mathrm{~d}, J=8.2 \mathrm{~Hz}, 1 \mathrm{H}), 6.78-6.71(\mathrm{~m}, 2 \mathrm{H}), 6.29(\mathrm{~s}, 1 \mathrm{H}), 5.68(\mathrm{~s}, 1 \mathrm{H}), 3.68(\mathrm{~s}, 3 \mathrm{H})$, $3.46(\mathrm{~s}, 1 \mathrm{H}), 2.62(\mathrm{~d}, J=13.9 \mathrm{~Hz}, 1 \mathrm{H}), 2.32$ (d, J = $13.8 \mathrm{~Hz}, 1 \mathrm{H}), 1.00(\mathrm{~s}, 9 \mathrm{H}), 0.22$ (s, 3H), $0.18(\mathrm{~s}, 3 \mathrm{H}) .{ }^{13} \mathrm{C}$ NMR $\left(125 \mathrm{MHz}, \mathrm{CDCl}_{3}\right) \delta 164.1,163.8,153.9,150.1,142.0$, 141.0, 135.0, 134.1, 130.6, 130.6, 130.1, 130.1, 130.1, 128.4, 123.1, 121.9, 118.6, $118.4,113.4,109.4,81.6,77.4,77.2,76.9,74.9,56.0,33.1,25.9,23.3,18.5,-4.2,-4.3$. ESI-HRMS: $[\mathrm{M}+\mathrm{H}]^{+}$calcd. For $\mathrm{C}_{31} \mathrm{H}_{35} \mathrm{CINO}_{9} \mathrm{SSi}^{+} \mathrm{m} / \mathrm{z}$ : 660.1485; found: 660.1482 . 

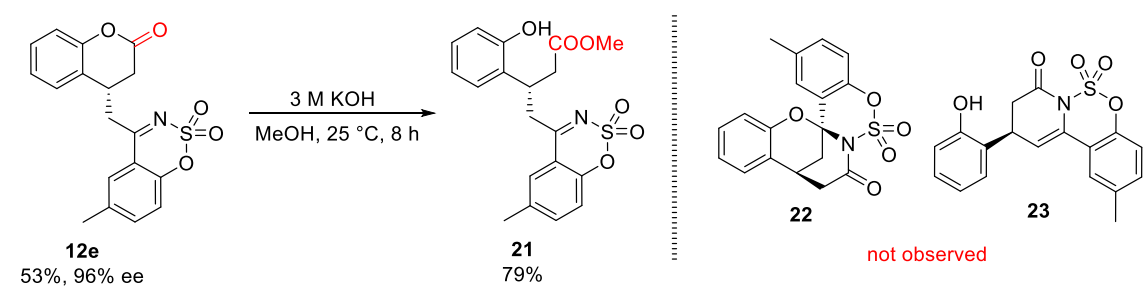

To a stirred solution of $12 \mathrm{e}$ in methanol being cooled to $0{ }^{\circ} \mathrm{C}$ was added about $5 \mu \mathrm{L} 3$ $\mathrm{M} \mathrm{KOH}$ in methanol until $\mathrm{pH}$ was 9. Then the reaction mixture was left at room temperature for $8 \mathrm{~h}$. After $12 \mathrm{e}$ was completely consumed, the reaction was quenched by $1 \mathrm{~N}$ aqueous $\mathrm{HCl}$. The aqueous phase was extracted by ethyl acetate for three times, combined the organic phase and reduced the solvent. The residue was purified with chromatography column (petroleum ether : ethyl acetate 3:1) to afford 21 (13 mg, 79\%). ${ }^{1} \mathrm{H}$ NMR (500 MHz, $\left.\mathrm{CDCl}_{3}\right) \delta 7.73(\mathrm{~s}, 1 \mathrm{H}), 7.46(\mathrm{~d}, J=8.4 \mathrm{~Hz}, 1 \mathrm{H})$, $7.15-7.04(\mathrm{~m}, 3 \mathrm{H}), 6.87(\mathrm{t}, J=7.4 \mathrm{~Hz}, 1 \mathrm{H}), 6.74(\mathrm{~d}, J=8.0 \mathrm{~Hz}, 1 \mathrm{H}), 6.26(\mathrm{~s}, 1 \mathrm{H}), 4.11-$ $4.04(\mathrm{~m}, 1 \mathrm{H}), 3.65(\mathrm{~d}, J=1.3 \mathrm{~Hz}, 3 \mathrm{H}), 3.38(\mathrm{~d}, J=7.0 \mathrm{~Hz}, 2 \mathrm{H}), 3.02$ (dd, $J=16.6,6.1 \mathrm{~Hz}$, $1 \mathrm{H}), 2.86(\mathrm{dd}, J=16.5,6.6 \mathrm{~Hz}, 1 \mathrm{H}), 2.39(\mathrm{~s}, 3 \mathrm{H}) .{ }^{13} \mathrm{C} \mathrm{NMR}\left(125 \mathrm{MHz}, \mathrm{CDCl}_{3}\right) \delta 179.1$, 173.7, 153.7, 151.6, 137.9, 136.2, 128.6, 128.1, 121.4, 118.9, 117.0, 116.2, 52.3, 41.1, 38.7, 33.9, 21.1. $[\alpha]_{D}{ }^{20}-17.10\left(c=1.00\right.$ in $\mathrm{CHCl}_{3}$ ). ESI-HRMS: $[\mathrm{M}+\mathrm{H}]^{+}$calcd. For $\mathrm{C}_{19} \mathrm{H}_{20} \mathrm{NO}_{6} \mathrm{~S}^{+} \mathrm{m} / \mathrm{z}$ : 390.1006; found: 390.1011 .

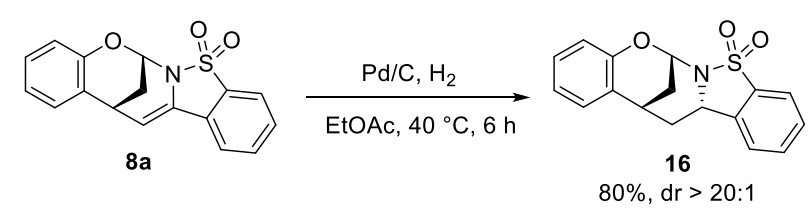

To a solution of $8 \mathrm{a}(15 \mathrm{mg}, 0.05 \mathrm{mmol}$ ) in EtOAc was added $1.5 \mathrm{mg} \mathrm{Pd} / \mathrm{C}$, then the reaction mixture kept stirring under 1 atm $\mathrm{H}_{2}$ atmosphere for $6 \mathrm{~h}$. After 8 a was completely consumed, the black suspension was filtered through celite and the product 16 was purified by chromatography column (petroleum ether : ethyl acetate 5:1) with a $80 \%$ yield as a white solid. ${ }^{1} \mathrm{H} \mathrm{NMR}\left(500 \mathrm{MHz} \mathrm{CDCl}_{3}\right) \delta 7.64(\mathrm{~d}, J=7.6 \mathrm{~Hz}$, 1H), $7.21(t, J=7.5 \mathrm{~Hz}, 1 \mathrm{H}), 7.10(\mathrm{t}, J=7.6 \mathrm{~Hz}, 1 \mathrm{H}), 7.01(\mathrm{~d}, J=7.2 \mathrm{~Hz}, 1 \mathrm{H}), 6.83-6.70$ (m, 2H), $6.65(\mathrm{~d}, J=7.7 \mathrm{~Hz}, 1 \mathrm{H}), 6.20(\mathrm{~d}, J=7.8 \mathrm{~Hz}, 1 \mathrm{H}), 6.03(\mathrm{~s}, 1 \mathrm{H}), 4.91(\mathrm{~d}, J=4.7 \mathrm{~Hz}$ 1H), $3.22(\mathrm{~s}, 1 \mathrm{H}), 2.79-2.66(\mathrm{~m}, 1 \mathrm{H}), 2.56(\mathrm{~d}, J=13.9 \mathrm{~Hz}, 1 \mathrm{H}), 2.24(\mathrm{~d}, J=13.3 \mathrm{~Hz}$, 1H), $2.14(\mathrm{~d}, J=13.3 \mathrm{~Hz}, 1 \mathrm{H}) .{ }^{13} \mathrm{C}$ NMR $\left(125 \mathrm{MHz}, \mathrm{CDCl}_{3}\right) \delta 152.2,140.7,138.0,131.6$, $128.3,127.9,127.7,125.2,122.2,121.0,120.4,116.0,78.9,52.2,32.7,26.8,25.7$. 
$[\alpha]_{D}^{20}+109.67\left(c=0.72\right.$ in $\left.\mathrm{CHCl}_{3}\right)$. ESI-HRMS: $[\mathrm{M}+\mathrm{H}]^{+}$calcd. For $\mathrm{C}_{17} \mathrm{H}_{16} \mathrm{NO}_{3} \mathrm{~S}^{+} \mathrm{m} / \mathrm{z}$ : 314.0845; found: 314.0847 . 
6. Single crystal X-ray diffraction data for compounds

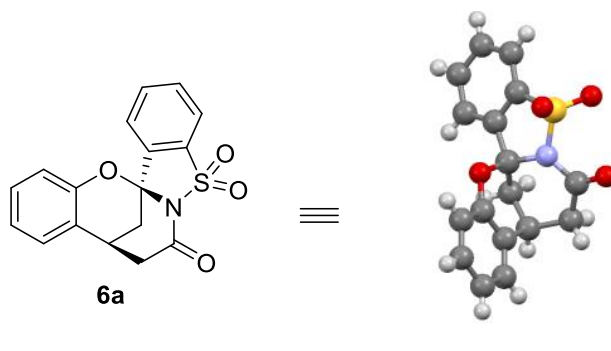

CCDC 1915200

Datablock lyk 0m - ellipsoid plot

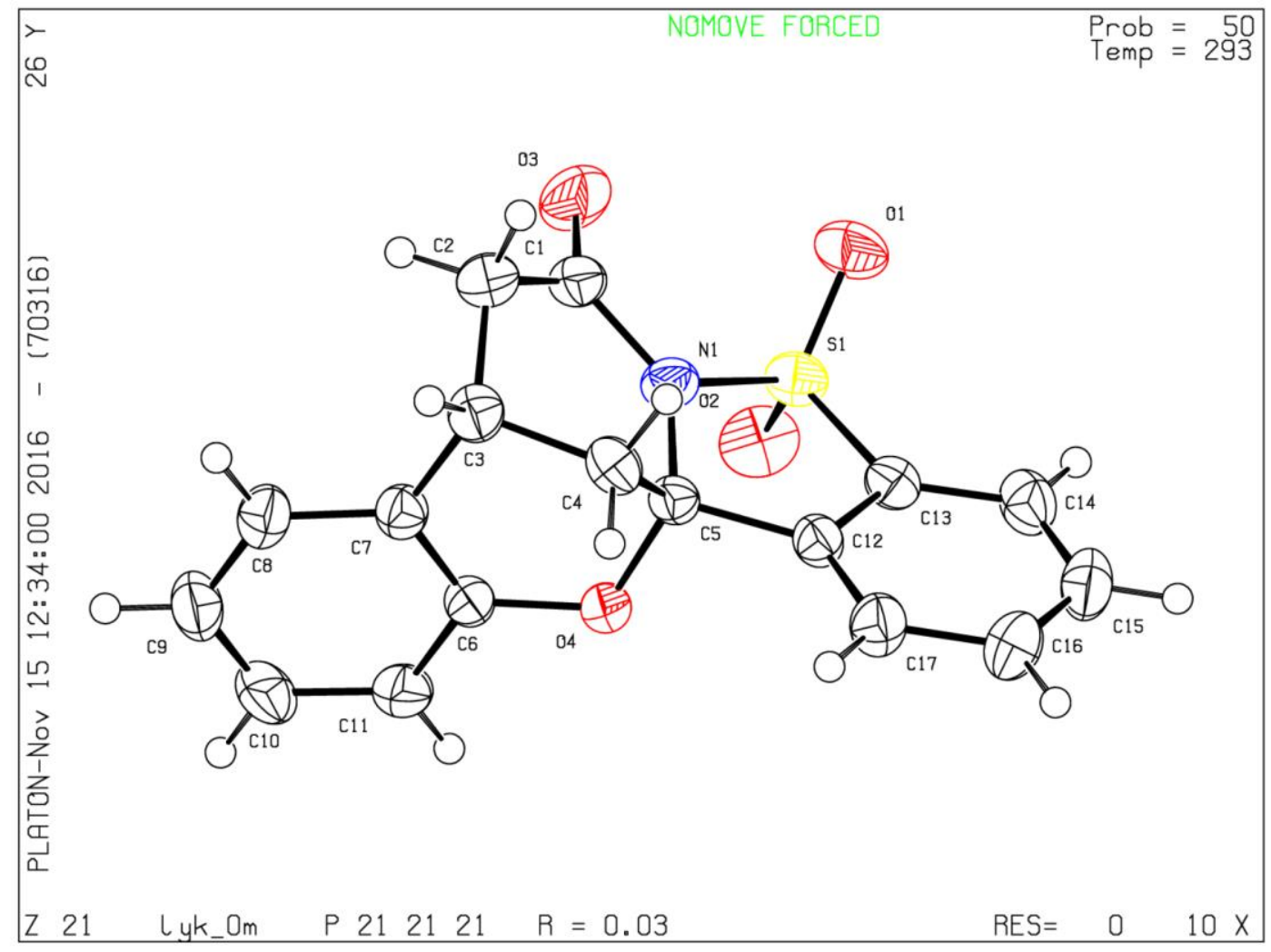




\section{Datablock: lyk_0m}

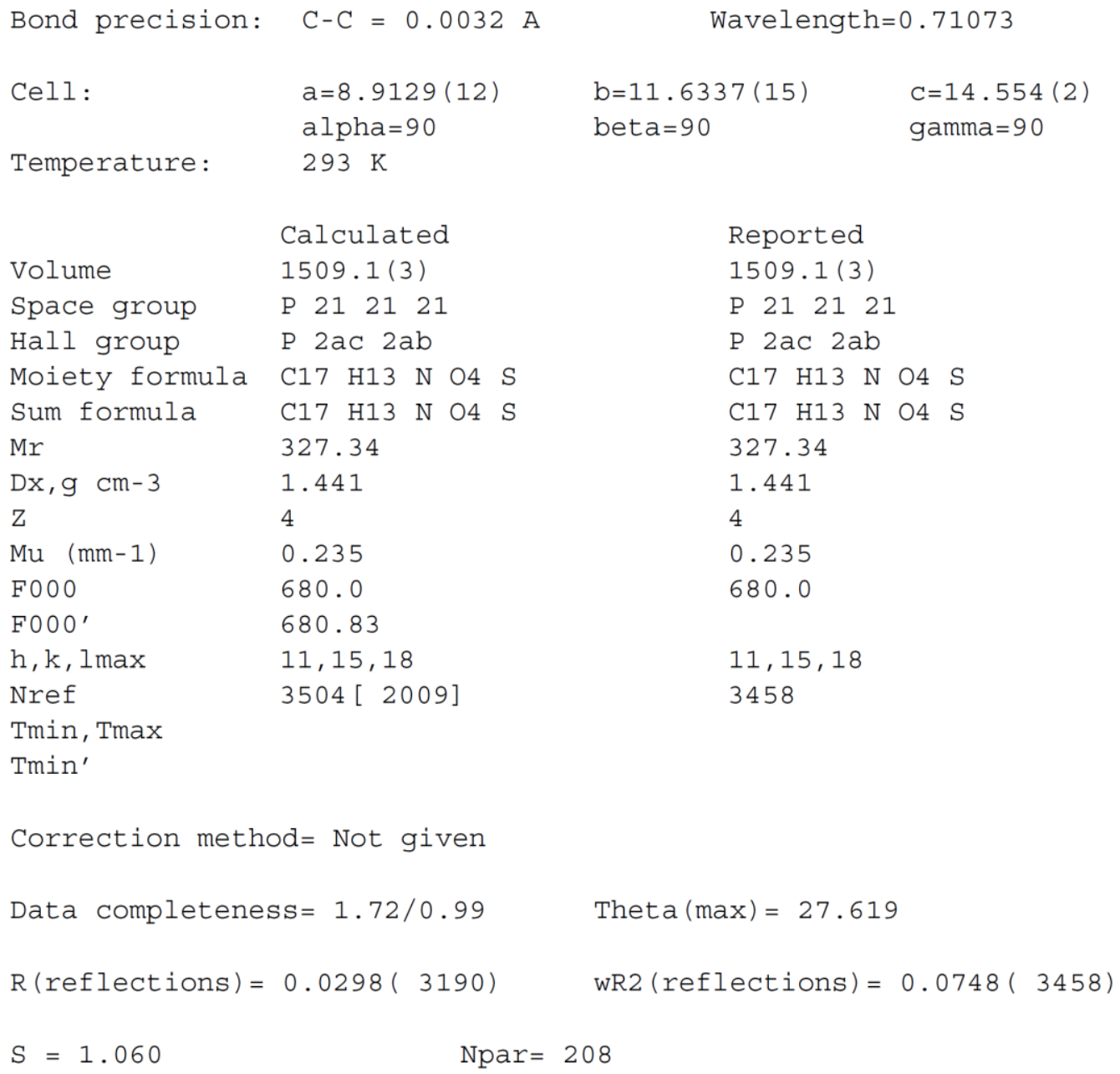

Tmin, Tmax

Tmin'

Correction method= Not given

Data completeness $=1.72 / 0.99$

Theta $(\max )=27.619$

$R($ reflections $)=0.0298(3190)$

WR2 (reflections $)=0.0748(3458)$

$S=1.060$

Npar $=208$ 


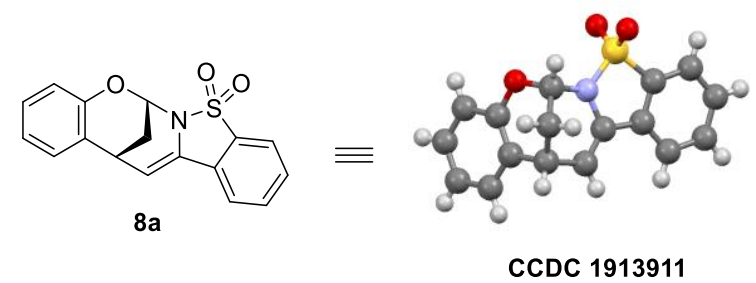

Datablock 180308lyk_0m - ellipsoid plot

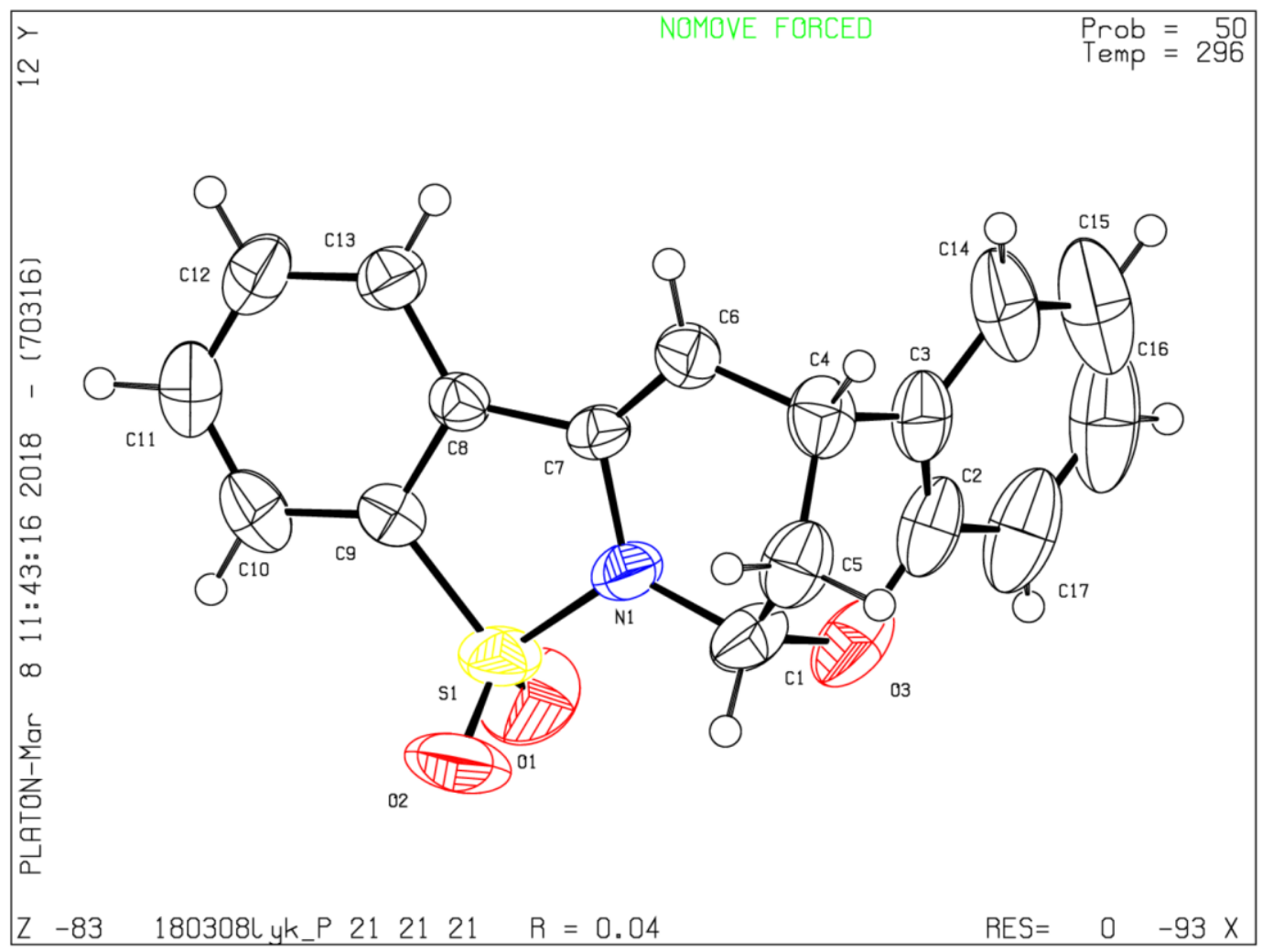




\section{Datablock: 180308lyk_0m}

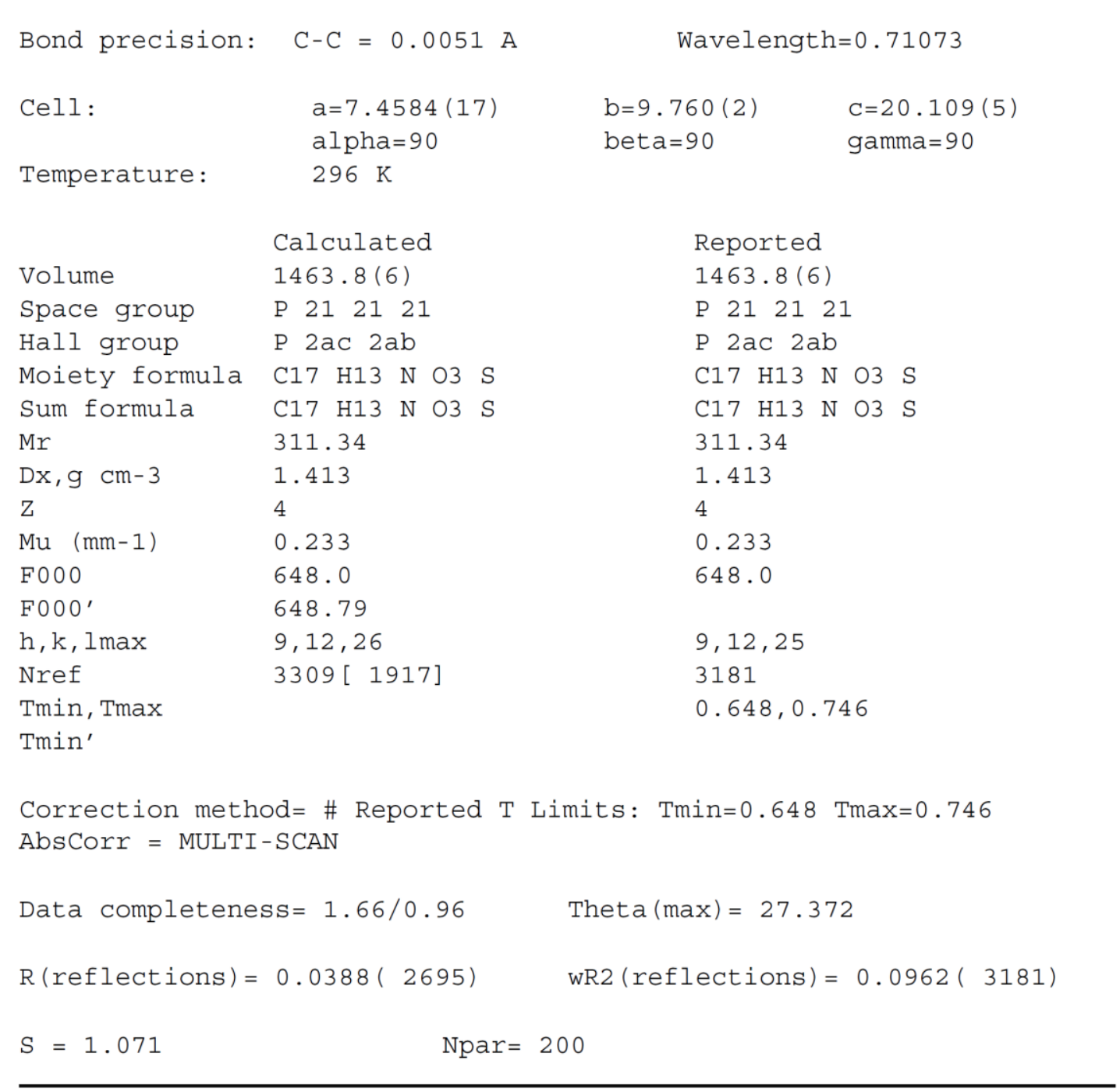




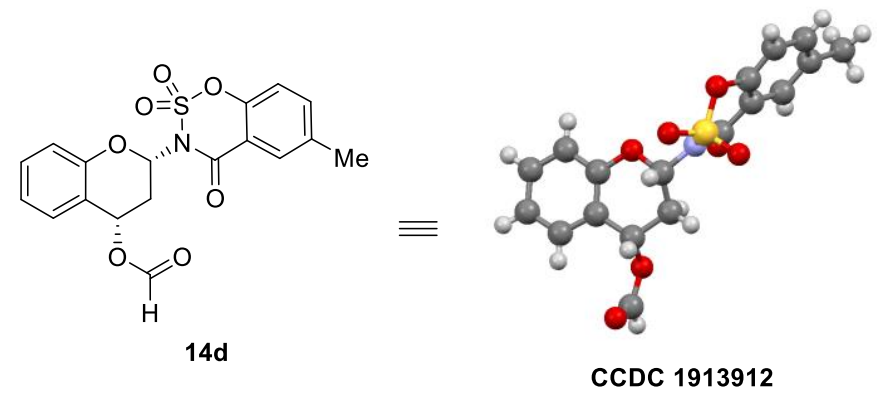

Datablock 181220cyh_0m - ellipsoid plot

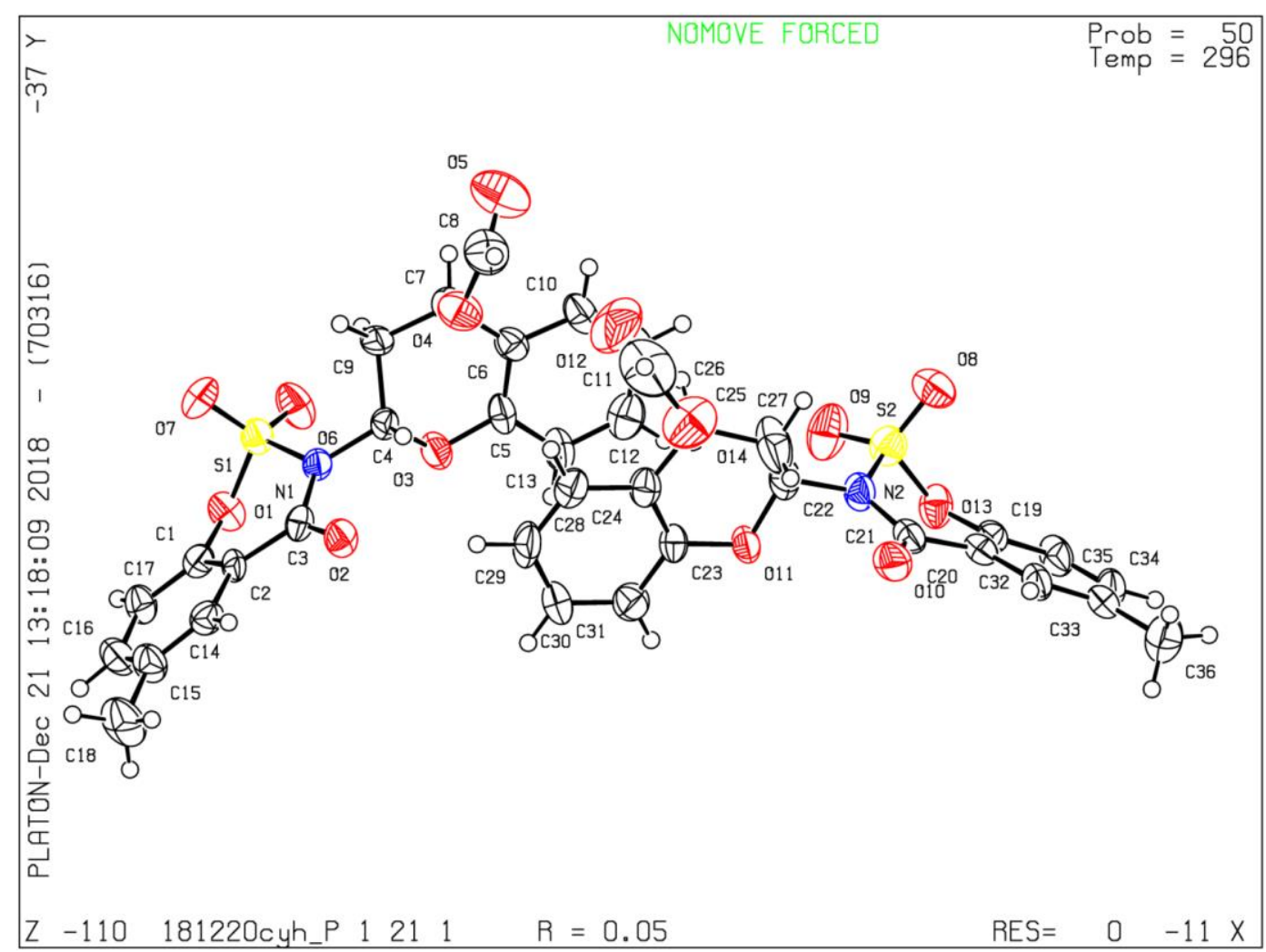




\section{Datablock: 181220cyh_0m}

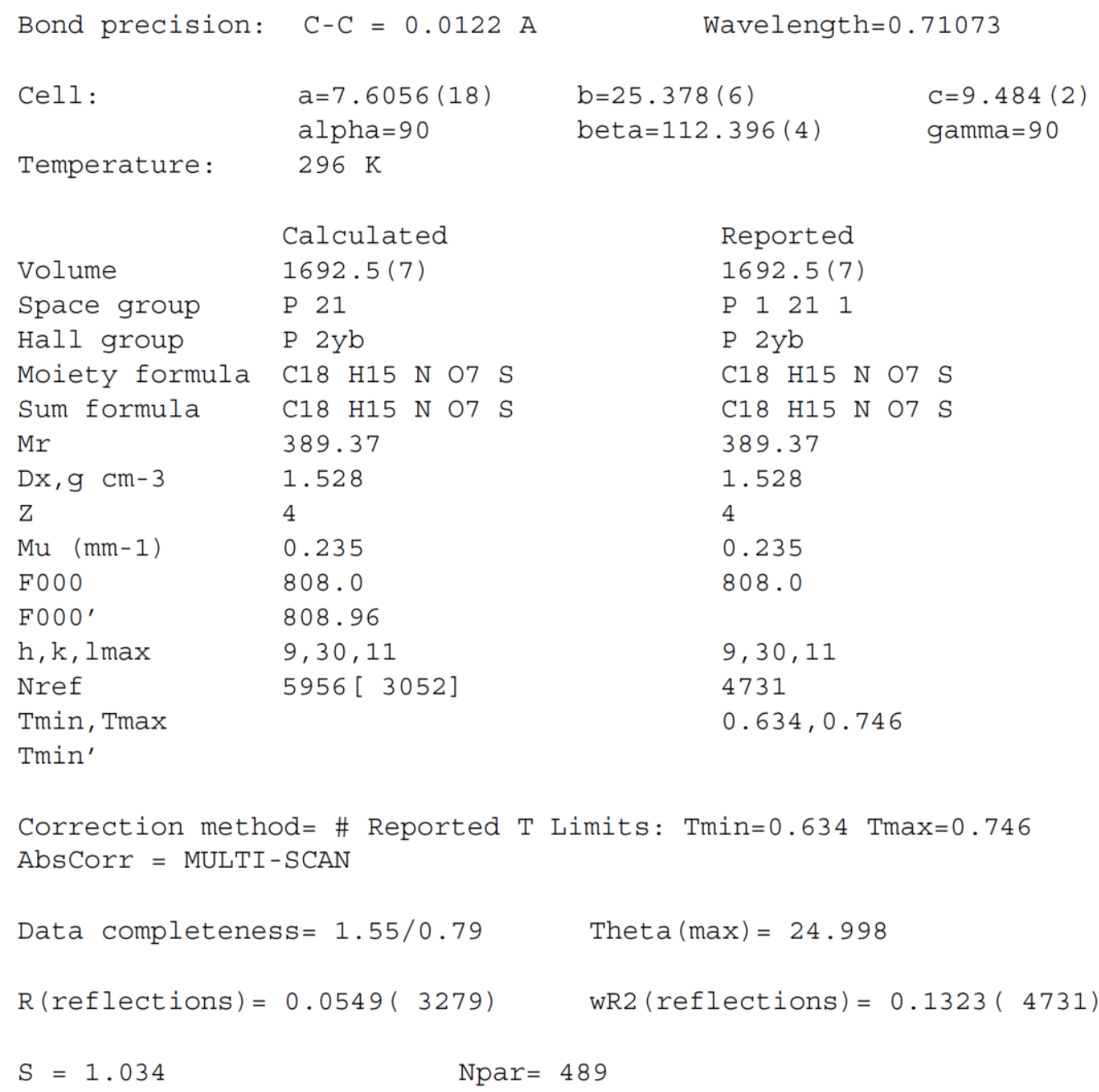




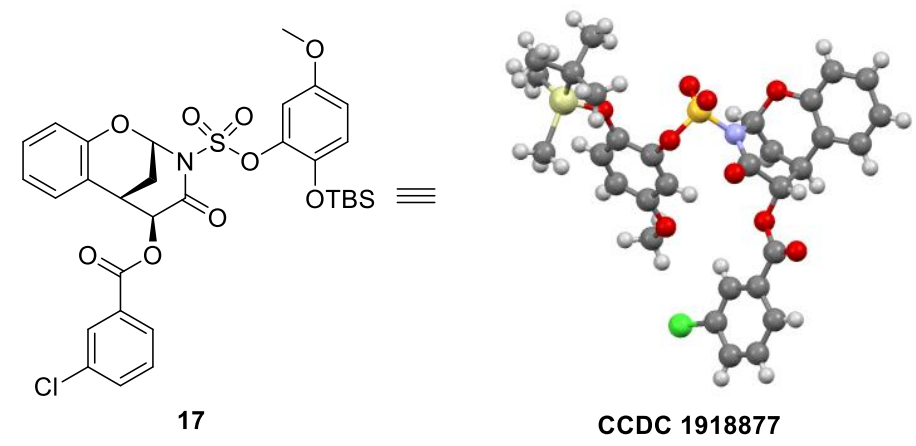

Datablock exp_10286_tw - ellipsoid plot

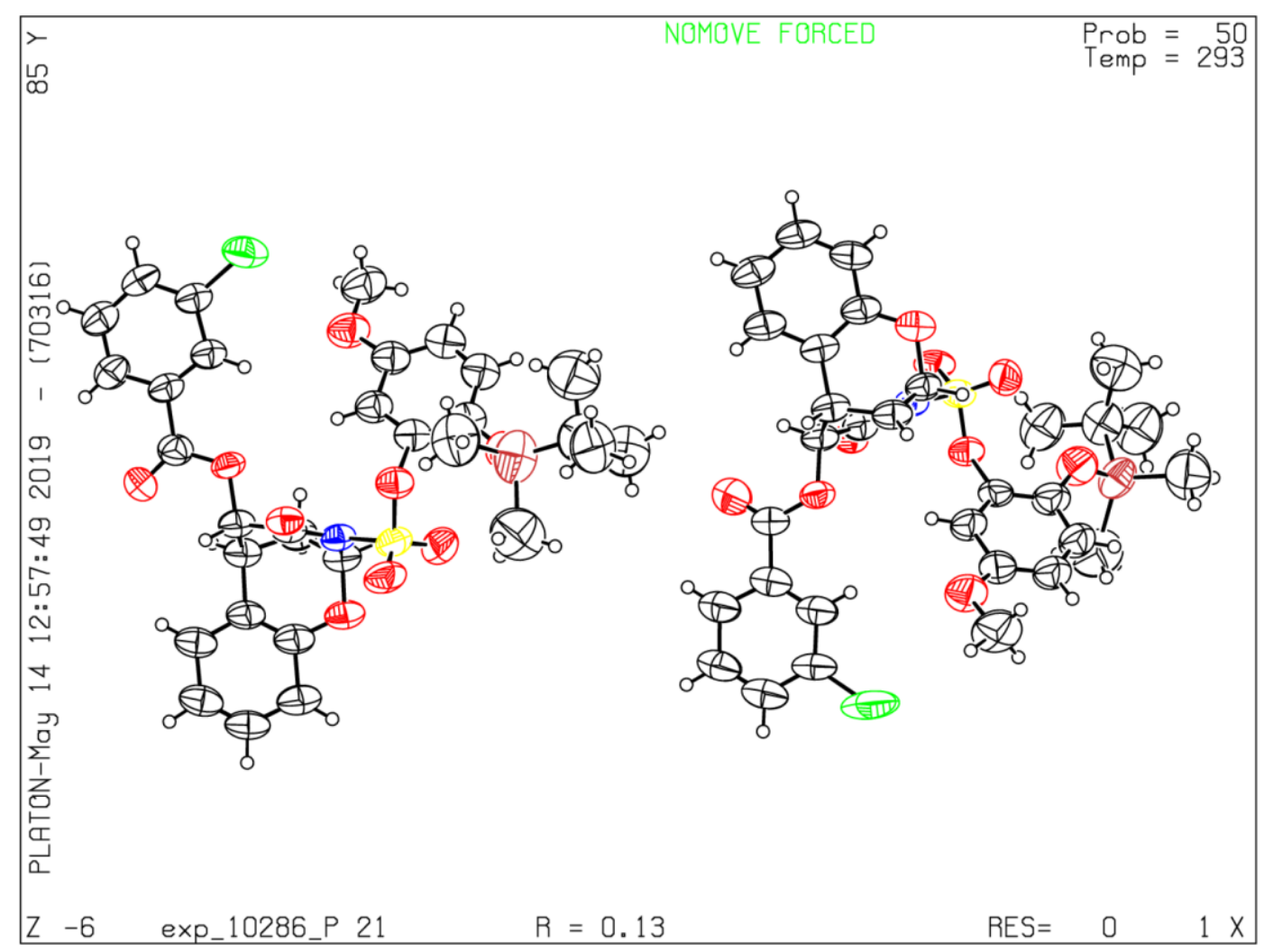




\section{Datablock: exp_10286_tw}

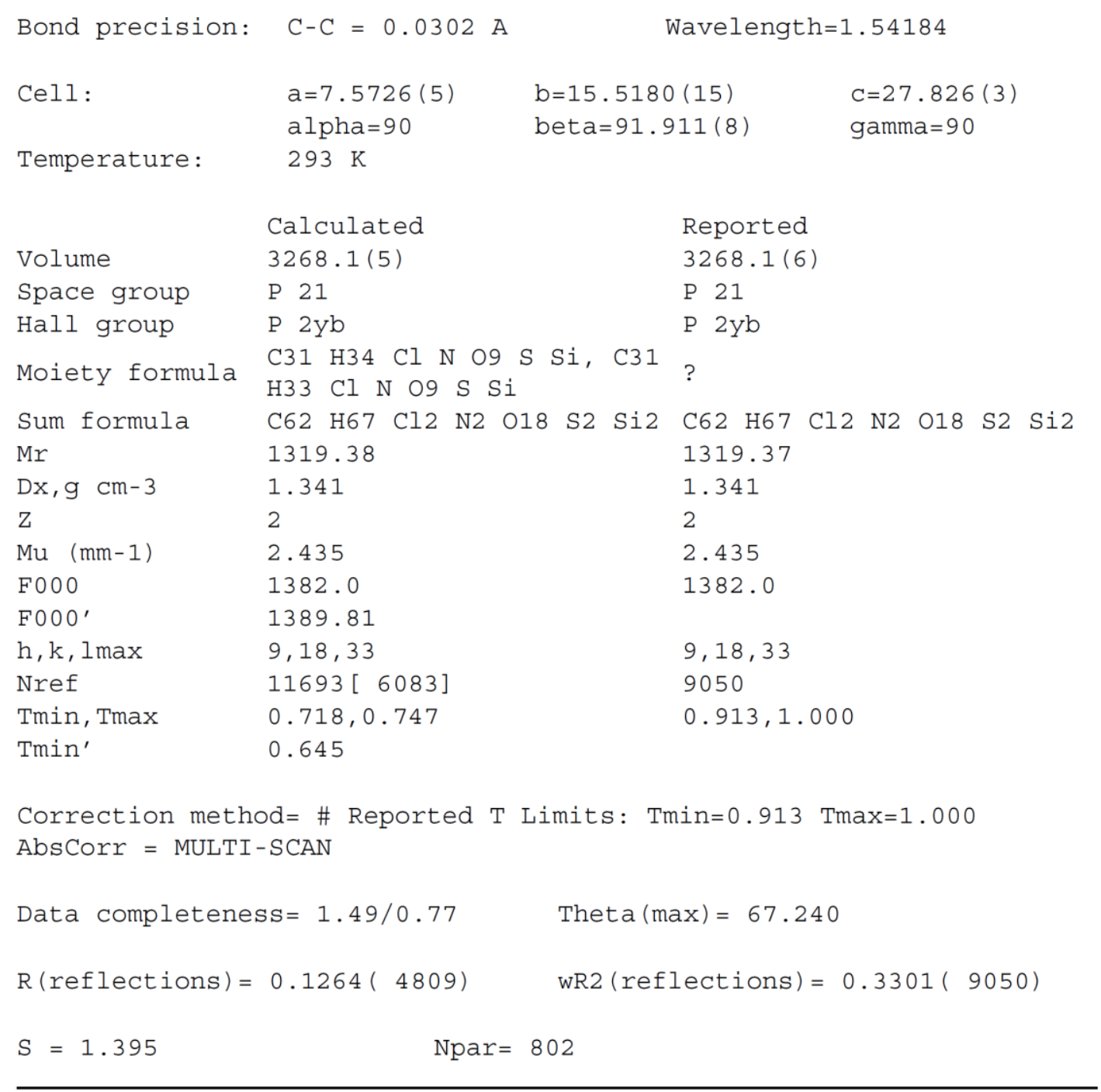




\section{References}

1. Wang, W.; Zu, L.; Zhang, S.; Hexin Xie, Catalytic Asymmetric oxa-Michael-Michael Cascade for Facile Construction of Chiral Chromans via an Aminal Intermediate. Org. Lett., 2009, 11, 1627-1630.

2. Jiang, C.; Lu, Y.; Hayashi, T., High Performance of a Palladium Phosphinooxazoline Catalyst in the Asymmetric Arylation of Cyclic N-Sulfonyl Ketimines. Angew. Chem. Int. Ed., 2014, 53, 9936-9939.

3. Zhang, Q.-R.; Huang, J.-R.; Zhang, W.; Dong, L., Highly Functionalized Pyridines Synthesis from N-Sulfonyl Ketimines and Alkynes Using the N-S Bond as an Internal Oxidant. Org. Lett., 2014, 16, 1684-1687.

4. Ramachary, D. B.; Shiva Prasad, M.; Vijaya Laxmi, S.; Madhavachary, R., Asymmetric synthesis of drug-like spiro[chroman-3,3'-indolin]-2'-ones through aminal-catalysis. Org. Biomol. Chem., 2014, 12, 574-580. 


\section{Products characterization}

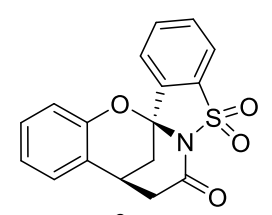

6 a

6a was synthesized following the general method ( $22 \mathrm{mg}, 66 \%$ yield) as a white solid. With $1 \mathrm{mmol}$ scale, $6 a$ (223 mg, 68\%) was obtained with excellent stereoselectivity. ${ }^{1} \mathrm{H}$ NMR $\left(500 \mathrm{MHz}, d_{\sigma}\right.$-DMSO) $\delta 8.14(\mathrm{~d}, J=7.8 \mathrm{~Hz}, 1 \mathrm{H}), 8.08(\mathrm{~d}, J=7.7 \mathrm{~Hz}, 1 \mathrm{H}), 7.97(\mathrm{t}$, $J=7.6 \mathrm{~Hz}, 1 \mathrm{H}), 7.89(\mathrm{t}, J=7.6 \mathrm{~Hz}, 1 \mathrm{H}), 7.36(\mathrm{~d}, J=7.5 \mathrm{~Hz}, 1 \mathrm{H}), 7.22(\mathrm{t}, J=7.2 \mathrm{~Hz}, 1 \mathrm{H})$, $7.05(\mathrm{t}, J=7.4 \mathrm{~Hz}, 1 \mathrm{H}), 6.83(\mathrm{~d}, J=8.1 \mathrm{~Hz}, 1 \mathrm{H}), 3.56$ (brs, 1H), 3.18 (ddd, J = 16.4, 15.2, $4.1 \mathrm{~Hz}, 2 \mathrm{H}), 2.75-2.58(\mathrm{~m}, 2 \mathrm{H}) .{ }^{13} \mathrm{C}$ NMR $\left(125 \mathrm{MHz}, d_{6}\right.$-DMSO) $\delta 167.6,150.7,135.5$, 134.7, 134.1, 133.0, 129.7, 129.3, 125.3, 125.2, 122.9, 121.7, 117.2, 110.0, 88.3, 42.7, 29.2, 28.8. $[\alpha]_{D}{ }^{20}-107.57\left(c=1.42\right.$ in $\mathrm{CHCl}_{3}$ ). ESI-HRMS: $[\mathrm{M}+\mathrm{H}]^{+}$calcd. For $\mathrm{C}_{17} \mathrm{H}_{14} \mathrm{NO}_{4} \mathrm{~S}^{+} \mathrm{m} / \mathrm{z}$ : 328.0638; found: 328.0635. The enantiomeric excess was determined by HPLC analysis on Daicel Chiralpak IA column ( $n$-hexane $/ i-\mathrm{PrOH} /$ dichloromethane $=70 / 27 / 3,1 \mathrm{~mL} / \mathrm{min}$ ), $\lambda=225 \mathrm{~nm}, t_{\text {major }}=9.75$ $\min , t_{\text {minor }}=11.39 \min$, ee $=97 \%$.

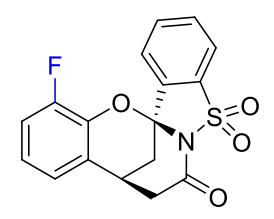

$6 b$

6b was synthesized following the general method ( $15 \mathrm{mg}, 44 \%$ yield) as a white solid. ${ }^{1} \mathrm{H}$ NMR $\left(500 \mathrm{MHz}, d_{\sigma}\right.$-DMSO) $\delta 8.17(\mathrm{~d}, J=7.8 \mathrm{~Hz}, 1 \mathrm{H}), 8.11(\mathrm{~d}, J=7.8 \mathrm{~Hz}, 1 \mathrm{H}), 8.00(\mathrm{t}$, $J=7.6 \mathrm{~Hz}, 1 \mathrm{H}), 7.91(\mathrm{t}, J=7.6 \mathrm{~Hz}, 1 \mathrm{H}), 7.25-7.14(\mathrm{~m}, 2 \mathrm{H}), 7.07-7.03(\mathrm{~m}, 1 \mathrm{H}), 3.65(\mathrm{~s}$, $1 \mathrm{H}), 3.28-3.16(\mathrm{~m}, 2 \mathrm{H}), 2.71(\mathrm{dd}, J=9.8,7.8 \mathrm{~Hz}, 2 \mathrm{H}) .{ }^{13} \mathrm{C} N M R\left(125 \mathrm{MHz}, d_{6}\right.$-DMSO) $\delta 167.4,150.8$ (d, $J=245.3 \mathrm{~Hz}), 139.0$ (d, $J=11.1 \mathrm{~Hz}), 135.7,134.2,133.2,128.0$, 125.4, 125.1 (d, $J=3.2 \mathrm{~Hz}), 122.9(\mathrm{~d}, J=7.1 \mathrm{~Hz}), 121.8,115.8(\mathrm{~d}, J=17.4 \mathrm{~Hz}), 88.2$, 42.4, 28.8, 28.7. $[\alpha]_{D}{ }^{20}-46.76\left(c=0.92\right.$ in $\left.\mathrm{CHCl}_{3}\right)$. ESI-HRMS: $[\mathrm{M}+\mathrm{H}]^{+}$calcd. For $\mathrm{C}_{17} \mathrm{H}_{13} \mathrm{FNO}_{4} \mathrm{~S}^{+} \mathrm{m} / \mathrm{z}$ : 346.0544; found: 346.0546. The enantiomeric excess was determined by HPLC analysis on Daicel Chiralpak IA column 
( $n$-hexane $/ i-\mathrm{PrOH} /$ dichloromethane $=65 / 32 / 3,1 \mathrm{~mL} / \mathrm{min}$ ), $\lambda=225 \mathrm{~nm}, t_{\text {major }}=13.95$ $\min , t_{\text {minor }}=16.47 \mathrm{~min}$, ee $=96 \%$.

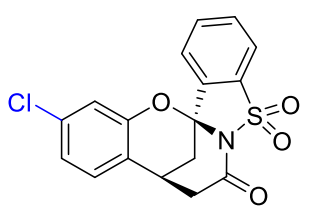

6c

6c was synthesized following the general method ( $21 \mathrm{mg}, 58 \%$ yield) as a white solid. ${ }^{1} \mathrm{H}$ NMR $\left(500 \mathrm{MHz}, d_{6}\right.$-DMSO) $\delta 8.15(\mathrm{~d}, J=7.8 \mathrm{~Hz}, 1 \mathrm{H}), 8.08(\mathrm{~d}, J=7.8 \mathrm{~Hz}, 1 \mathrm{H}), 8.02-$ $7.95(\mathrm{~m}, 1 \mathrm{H}), 7.93-7.86(\mathrm{~m}, 1 \mathrm{H}), 7.41(\mathrm{~d}, J=8.3 \mathrm{~Hz}, 1 \mathrm{H}), 7.12(\mathrm{dd}, J=8.2,2.0 \mathrm{~Hz}, 1 \mathrm{H})$ $6.99(\mathrm{~d}, J=2.0 \mathrm{~Hz}, 1 \mathrm{H}), 3.60(\mathrm{~s}, 1 \mathrm{H}), 3.18(\mathrm{ddd}, J=16.3,15.4,4.1 \mathrm{~Hz}, 2 \mathrm{H}), 2.75-2.62$ (m, 2H). ${ }^{13} \mathrm{C}$ NMR $\left(125 \mathrm{MHz}, d_{6}\right.$-DMSO) $\delta 167.4,151.5,135.6,134.3,134.1,133.2$, 133.2, 131.3, 125.4, 124.4, 123.0, 121.8, 117.10, 88.3, 42.4, 28.9, 28.5. $[\alpha]_{D}{ }^{20}-82.65$ ( $c=1.17$ in $\mathrm{CHCl}_{3}$ ). ESI-HRMS: $[\mathrm{M}+\mathrm{H}]^{+}$calcd. For $\mathrm{C}_{17} \mathrm{H}_{13} \mathrm{ClNO}_{4} \mathrm{~S}^{+} \mathrm{m} / \mathrm{z}$ : 362.0248; found: 362.0252. The enantiomeric excess was determined by HPLC analysis on Daicel Chiralpak IA column ( $n$-hexane $/ i-\mathrm{PrOH} /$ dichloromethane $=65 / 32 / 3,1 \mathrm{~mL} / \mathrm{min}$ ), $\lambda=$ $225 \mathrm{~nm}, t_{\text {major }}=12.61 \mathrm{~min}, t_{\text {minor }}=15.39 \mathrm{~min}, \mathrm{ee}=95 \%$.

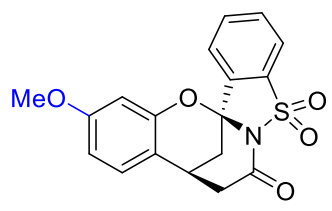

6d

6d was synthesized following the general method (19 $\mathrm{mg}, 54 \%$ yield) as a white solid. ${ }^{1} \mathrm{H}$ NMR $\left(500 \mathrm{MHz}, d_{6}\right.$-DMSO) $\delta 8.13(\mathrm{~d}, J=7.8 \mathrm{~Hz}, 1 \mathrm{H}), 8.07(\mathrm{~d}, J=7.7 \mathrm{~Hz}, 1 \mathrm{H}), 7.97(\mathrm{t}$, $J=7.6 \mathrm{~Hz}, 1 \mathrm{H}), 7.88(\mathrm{t}, J=7.6 \mathrm{~Hz}, 1 \mathrm{H}), 7.25(\mathrm{~d}, J=8.5 \mathrm{~Hz}, 1 \mathrm{H}), 6.64(\mathrm{dd}, J=8.5,2.4 \mathrm{~Hz}$, 1H), $6.44(\mathrm{~d}, J=2.3 \mathrm{~Hz}, 1 \mathrm{H}), 3.68(\mathrm{~s}, 3 \mathrm{H}), 3.50(\mathrm{~s}, 1 \mathrm{H}), 3.13(\mathrm{ddd}, J=16.6,15.2,4.1 \mathrm{~Hz}$, 2H), $2.72-2.58(\mathrm{~m}, 2 \mathrm{H}) .{ }^{13} \mathrm{C}$ NMR (125 MHz, $d_{6}$-DMSO) $\delta 167.8,160.2,151.5,135.5$, 134.7, 134.2, 133.0, 130.3, 125.3, 121.7, 117.1, 110.1, 101.8, 88.3, 55.7, 42.9, 29.5, 28.2. $[\alpha]_{D}{ }^{20}-66.53\left(c=1.33\right.$ in $\left.\mathrm{CHCl}_{3}\right)$. ESI-HRMS: $[\mathrm{M}+\mathrm{H}]^{+}$calcd. For $\mathrm{C}_{18} \mathrm{H}_{16} \mathrm{NO}_{5} \mathrm{~S}^{+} \mathrm{m} / \mathrm{z}$ : 358.0744; found: 358.0743. The enantiomeric excess was determined by HPLC analysis on Daicel Chiralpak IA column ( $n$-hexane/i-PrOH/dichloromethane $=65 / 32 / 3$ $1 \mathrm{~mL} / \mathrm{min}), \lambda=225 \mathrm{~nm}, t_{\text {major }}=17.01 \mathrm{~min}, t_{\text {minor }}=20.43 \mathrm{~min}$, ee $=94 \%$. 


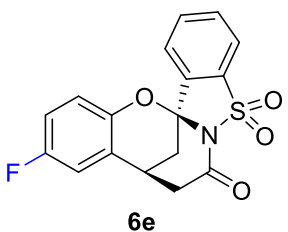

6e was synthesized following the general method ( $21 \mathrm{mg}, 62 \%$ yield) as a white solid. ${ }^{1} \mathrm{H}$ NMR $\left(500 \mathrm{MHz}, d_{6}\right.$-DMSO) $\delta 8.14(\mathrm{~d}, J=7.8 \mathrm{~Hz}, 1 \mathrm{H}), 8.07(\mathrm{~d}, J=7.7 \mathrm{~Hz}, 1 \mathrm{H}), 8.04-$ $7.94(\mathrm{~m}, 1 \mathrm{H}), 7.94-7.86(\mathrm{~m}, 1 \mathrm{H}), 7.27$ (dd, J = 8.9, 3.0 Hz, 1H), 7.07 (td, J = 8.7, $3.1 \mathrm{~Hz}$, $1 \mathrm{H}), 6.88(\mathrm{dd}, J=9.0,4.7 \mathrm{~Hz}, 1 \mathrm{H}), 3.58(\mathrm{~s}, 1 \mathrm{H}), 3.21(\mathrm{dd}, J=17.4,5.1 \mathrm{~Hz}, 1 \mathrm{H}), 3.18-$ $3.09(\mathrm{~m}, 1 \mathrm{H}), 2.74(\mathrm{~d}, J=17.4 \mathrm{~Hz}, 1 \mathrm{H}), 2.64(\mathrm{dd}, J=13.3,1.8 \mathrm{~Hz}, 1 \mathrm{H}) .{ }^{13} \mathrm{C}$ NMR $(125$ MHz, $d_{6}$-DMSO) $\delta 167.4,157.6(d, J=238.5 \mathrm{~Hz}), 146.9(\mathrm{~d}, J=1.8 \mathrm{~Hz}), 135.6,134.5$, 134.1, 133.1, $126.8(\mathrm{~d}, J=7.8 \mathrm{~Hz}), 125.3,121.7,118.8(\mathrm{~d}, J=8.3 \mathrm{~Hz}), 116.2(\mathrm{~d}, J=$ $23.5 \mathrm{~Hz}), 115.8(\mathrm{~d}, J=23.3 \mathrm{~Hz}), 88.3,42.3,29.0,28.9 .[\alpha]_{D}{ }^{20}-75.15\left(c=1.42\right.$ in $\left.\mathrm{CHCl}_{3}\right)$. ESI-HRMS: $[\mathrm{M}+\mathrm{H}]^{+}$calcd. For $\mathrm{C}_{17} \mathrm{H}_{13} \mathrm{FNO}_{4} \mathrm{~S}^{+} \mathrm{m} / \mathrm{z}$ : 346.0544; found: 346.0540 . The enantiomeric excess was determined by HPLC analysis on Daicel Chiralpak IA column ( $n$-hexane $/ \mathrm{i}$-PrOH/dichloromethane $=70 / 27 / 3,1 \mathrm{~mL} / \mathrm{min}$ ), $\lambda=225 \mathrm{~nm}, t_{\text {major }}=19.43$ $\min , t_{\text {minor }}=22.29 \min$, ee $=94 \%$.

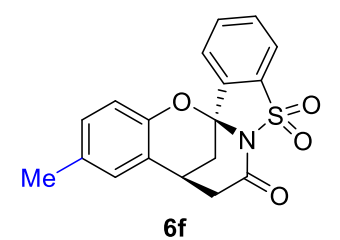

6f was synthesized following the general method ( $24 \mathrm{mg}, 71 \%$ yield) as a white solid. ${ }^{1} \mathrm{H} \mathrm{NMR}\left(500 \mathrm{MHz}, \mathrm{CDCl}_{3}\right) \delta 7.87(\mathrm{~d}, J=7.8 \mathrm{~Hz}, 1 \mathrm{H}), 7.80(\mathrm{t}, J=7.6 \mathrm{~Hz}, 1 \mathrm{H}), 7.73(\mathrm{t}, J=$ $7.6 \mathrm{~Hz}, 1 \mathrm{H}), 7.66(\mathrm{~d}, J=7.7 \mathrm{~Hz}, 1 \mathrm{H}), 6.99(\mathrm{~d}, J=8.3 \mathrm{~Hz}, 1 \mathrm{H}), 6.95(\mathrm{~s}, 1 \mathrm{H}), 6.72(\mathrm{~d}, J=$ $8.3 \mathrm{~Hz}, 1 \mathrm{H}), 3.46(\mathrm{~s}, 1 \mathrm{H}), 2.99(\mathrm{dd}, J=17.5,5.2 \mathrm{~Hz}, 1 \mathrm{H}), 2.95-2.81(\mathrm{~m}, 2 \mathrm{H}), 2.43(\mathrm{dd}, J$ $=13.0,2.0 \mathrm{~Hz}, 1 \mathrm{H}), 2.29$ (s, 3H). ${ }^{13} \mathrm{C} \mathrm{NMR}\left(125 \mathrm{MHz}, \mathrm{CDCl}_{3}\right) \delta 166.5,148.3,134.7$, $134.5,134.4,132.3,131.9,130.0,128.8,123.9,123.2,121.6,117.4,87.4,42.5,30.6$, 29.3, 20.5. $[\alpha]_{D}{ }^{20}-91.95\left(c=1.67\right.$ in $\left.\mathrm{CHCl}_{3}\right)$. ESI-HRMS: $[\mathrm{M}+\mathrm{H}]^{+}$calcd. For $\mathrm{C}_{18} \mathrm{H}_{16} \mathrm{NO}_{4} \mathrm{~S}^{+}$ m/z: 342.0795; found: 342.0800. The enantiomeric excess was determined by HPLC analysis on Daicel Chiralpak IA column ( $n$-hexane/i-PrOH/dichloromethane = 70/27/3, $1 \mathrm{~mL} / \mathrm{min}), \lambda=225 \mathrm{~nm}, t_{\text {major }}=15.55 \mathrm{~min}, t_{\text {minor }}=18.01 \mathrm{~min}$, ee $=96 \%$. 


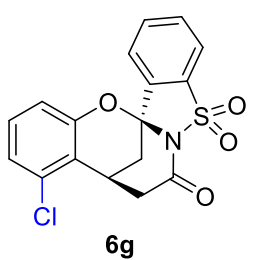

$6 \mathrm{~g}$ was synthesized following the general method ( $21 \mathrm{mg}, 58 \%$ yield) as a white solid. ${ }^{1} \mathrm{H}$ NMR $\left(500 \mathrm{MHz}, d_{\sigma}\right.$-DMSO) $\delta 8.16(\mathrm{~d}, J=7.8 \mathrm{~Hz}, 1 \mathrm{H}), 8.08(\mathrm{~d}, J=7.8 \mathrm{~Hz}, 1 \mathrm{H}), 7.99(\mathrm{t}$, $J=7.5 \mathrm{~Hz}, 1 \mathrm{H}), 7.90(\mathrm{t}, J=7.6 \mathrm{~Hz}, 1 \mathrm{H}), 7.28(\mathrm{t}, J=8.1 \mathrm{~Hz}, 1 \mathrm{H}), 7.22(\mathrm{~d}, J=7.7 \mathrm{~Hz}, 1 \mathrm{H})$, $6.89(\mathrm{~d}, J=8.1 \mathrm{~Hz}, 1 \mathrm{H}$ ), $3.78(\mathrm{~s}, 1 \mathrm{H}), 3.28$ (dd, $J=17.5,5.2 \mathrm{~Hz}, 1 \mathrm{H}$ ), 3.17 (dd, $J=13.4$, $3.1 \mathrm{~Hz}, 1 \mathrm{H}), 2.78-2.63(\mathrm{~m}, 2 \mathrm{H}) .{ }^{13} \mathrm{C}$ NMR (125 MHz, $d_{6}$-DMSO) $\delta 167.2,152.0,135.7$, 134.2, 134.1, 133.2, 133.1, 130.3, 125.3, 123.4, 122.8, 121.8, 116.6, 88.2, 29.0, 27.1. $[\alpha]_{D}{ }^{20}-184.93\left(c=0.32\right.$ in $\left.\mathrm{CHCl}_{3}\right)$. ESI-HRMS: $[\mathrm{M}+\mathrm{H}]^{+}$calcd. For $\mathrm{C}_{17} \mathrm{H}_{13} \mathrm{ClNO}_{4} \mathrm{~S}^{+} \mathrm{m} / \mathrm{z}$ : 362.0248; found: 362.0245. The enantiomeric excess was determined by HPLC analysis on Daicel Chiralpak IA column ( $n$-hexane/i-PrOH =70/30, $1 \mathrm{~mL} / \mathrm{min}$ ), $\lambda=210$ $\mathrm{nm}, t_{\text {major }}=19.32 \mathrm{~min}, t_{\text {minor }}=24.94 \mathrm{~min}$, ee $=97 \%$.

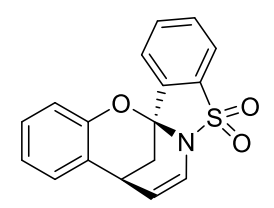

$7 \mathbf{a}$

7a (21 mg, 68\% yield), a white solid,.was catalyzed with $p$-toluenesulfonic acid (7.3 mg, 0.4 equiv) at $25{ }^{\circ} \mathrm{C}$ for only $0.5 \mathrm{~h} .{ }^{1} \mathrm{H}$ NMR $\left(500 \mathrm{MHz}, \mathrm{CDCl}_{3}\right) \delta 7.89(\mathrm{~d}, J=7.7 \mathrm{~Hz}$, 1H), $7.79(t, J=7.6 \mathrm{~Hz}, 1 \mathrm{H}), 7.75-7.65(\mathrm{~m}, 2 \mathrm{H}), 7.18-7.12(\mathrm{~m}, 2 \mathrm{H}), 6.95(\mathrm{t}, J=7.4 \mathrm{~Hz}$, 1H), $6.90(\mathrm{~d}, J=8.4 \mathrm{~Hz}, 1 \mathrm{H}), 6.69(\mathrm{dd}, J=7.7,0.5 \mathrm{~Hz}, 1 \mathrm{H}), 5.63(\mathrm{t}, J=7.3 \mathrm{~Hz}, 1 \mathrm{H}), 3.75$ $-3.63(\mathrm{~m}, 1 \mathrm{H}), 2.69-2.56(\mathrm{~m}, 1 \mathrm{H}), 2.01(\mathrm{dd}, J=12.5,2.0 \mathrm{~Hz}, 1 \mathrm{H}) .{ }^{13} \mathrm{C} N M R(125 \mathrm{MHz}$, $\left.\mathrm{CDCl}_{3}\right) \delta 151.7,136.5,134.9,134.0,131.4,128.0,127.6,125.3,124.4,121.6,121.2$, $119.6,117.0,111.7,86.4,30.3,29.6$. $[\alpha]_{D}^{20}+85.47\left(c=0.58\right.$ in $\mathrm{CHCl}_{3}$ ). ESI-HRMS: $[\mathrm{M}+\mathrm{H}]^{+}$calcd. For $\mathrm{C}_{17} \mathrm{H}_{14} \mathrm{NO}_{3} \mathrm{~S}^{+} \mathrm{m} / \mathrm{z}$ : 312.0689; found: 312.0694 . 


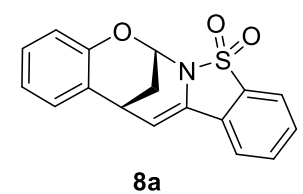

8a was synthesized following the general method ( $23 \mathrm{mg}, 74 \%$ yield) as a white solid. With $1 \mathrm{mmol}$ scale, $8 \mathrm{a}$ ( $244 \mathrm{mg}, 79 \%$ ) was obtained with excellent stereoselectivity. ${ }^{1} \mathrm{H}$ NMR $\left(500 \mathrm{MHz}, \mathrm{CDCl}_{3}\right) \delta 7.80(\mathrm{~d}, J=7.7 \mathrm{~Hz}, 1 \mathrm{H}), 7.63-7.46(\mathrm{~m}, 3 \mathrm{H}), 7.13(\mathrm{t}, J=$ $7.8 \mathrm{~Hz}, 2 \mathrm{H}), 7.00(\mathrm{~d}, J=8.1 \mathrm{~Hz}, 1 \mathrm{H}), 6.89(\mathrm{t}, J=7.4 \mathrm{~Hz}, 1 \mathrm{H}), 6.39(\mathrm{~s}, 1 \mathrm{H}), 6.09(\mathrm{~d}, J=7.3$ $\mathrm{Hz}, 1 \mathrm{H}), 3.73(\mathrm{~d}, J=7.1 \mathrm{~Hz}, 1 \mathrm{H}), 2.40(\mathrm{~d}, J=12.8 \mathrm{~Hz}, 1 \mathrm{H}), 2.06(\mathrm{~d}, J=12.6 \mathrm{~Hz}, 1 \mathrm{H}) .{ }^{13} \mathrm{C}$ $\operatorname{NMR}\left(125 \mathrm{MHz}, \mathrm{CDCl}_{3}\right) \delta 151.3,133.0,132.6,130.0,129.9,128.5,128.2,128.1,124.7$, $121.3,121.2,121.0,117.8,104.3,72.8,27.6,26.3 .[\alpha]_{D}{ }^{20}+153.06\left(c=1.00\right.$ in $\left.\mathrm{CHCl}_{3}\right)$. ESI-HRMS: $[\mathrm{M}+\mathrm{H}]^{+}$calcd. For $\mathrm{C}_{17} \mathrm{H}_{14} \mathrm{NO}_{3} \mathrm{~S}^{+} \mathrm{m} / \mathrm{z}$ : 312.0689; found: 312.0687 . The enantiomeric excess was determined by HPLC analysis on Daicel Chiralpak IB column $(n$-hexane $/ i-\operatorname{PrOH}=70 / 30,1 \mathrm{~mL} / \mathrm{min}), \lambda=216 \mathrm{~nm}, t_{\text {major }}=9.78 \mathrm{~min}, t_{\text {minor }}=12.12 \mathrm{~min}$, ee $=95 \%$.

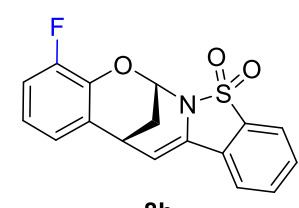

$8 b$

8b was synthesized following the general method ( $19 \mathrm{mg}, 58 \%$ yield) as a white solid. ${ }^{1} \mathrm{H}$ NMR $\left(500 \mathrm{MHz}, \mathrm{CDCl}_{3}\right) \delta 7.79(\mathrm{~d}, J=7.8 \mathrm{~Hz}, 1 \mathrm{H}), 7.63-7.56(\mathrm{~m}, 2 \mathrm{H}), 7.56-7.49$ $(m, 1 H), 6.95-6.87(m, 2 H), 6.84-6.80(m, 1 H), 6.47(d, J=0.9 H z, 1 H), 6.08(d, J=$ $7.3 \mathrm{~Hz}, 1 \mathrm{H}), 3.81-3.74(\mathrm{~m}, 1 \mathrm{H}), 2.40(\mathrm{~d}, J=13.0 \mathrm{~Hz}, 1 \mathrm{H}), 2.08-2.04(\mathrm{~m}, 1 \mathrm{H}) .{ }^{13} \mathrm{C}$ $\operatorname{NMR}\left(125 \mathrm{MHz}, \mathrm{CDCl}_{3}\right) \delta 151.9(\mathrm{~d}, J=247.8 \mathrm{~Hz}), 139.6(\mathrm{~d}, J=11.3 \mathrm{~Hz}), 133.2,132.5$, $130.2,130.1,128.3,127.4,123.1(\mathrm{~d}, J=3.5 \mathrm{~Hz}), 121.3,121.1,121.0(\mathrm{~d}, J=6.9 \mathrm{~Hz}$ ), $115.0(\mathrm{~d}, J=18.1 \mathrm{~Hz}), 103.7,72.8,27.2,26.0 .[\alpha]_{\mathrm{D}}^{20}+155.15\left(c=1.25\right.$ in $\left.\mathrm{CHCl}_{3}\right)$. ESI-HRMS: $[\mathrm{M}+\mathrm{H}]^{+}$calcd. For $\mathrm{C}_{17} \mathrm{H}_{13} \mathrm{FNO}_{3} \mathrm{~S}^{+} \mathrm{m} / \mathrm{z}$ : 330.0595; found: 330.0597 . The enantiomeric excess was determined by HPLC analysis on Daicel Chiralpak IB column ( $n$-hexane $/ \mathrm{i}-\mathrm{PrOH} /$ dichloromethane $=70 / 27 / 3,1 \mathrm{~mL} / \mathrm{min}$ ), $\lambda=216 \mathrm{~nm}, t_{\text {major }}=10.60$ $\min , t_{\text {minor }}=11.65 \min$, ee $=96 \%$. 


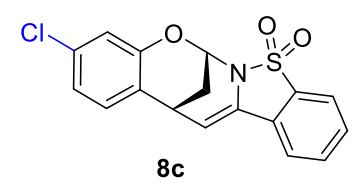

8c was synthesized following the general method (18 $\mathrm{mg}, 52 \%$ yield) as a white solid. ${ }^{1} \mathrm{H}$ NMR $\left(500 \mathrm{MHz}, \mathrm{CDCl}_{3}\right) \delta 7.80(\mathrm{~d}, J=7.8 \mathrm{~Hz}, 1 \mathrm{H}), 7.63-7.55(\mathrm{~m}, 2 \mathrm{H}), 7.55-7.49$ (m, 1H), $7.02(\mathrm{dd}, J=15.0,5.0 \mathrm{~Hz}, 2 \mathrm{H}), 6.86(\mathrm{dd}, J=8.1,2.0 \mathrm{~Hz}, 1 \mathrm{H}), 6.37(\mathrm{~d}, J=1.0 \mathrm{~Hz}$, $1 \mathrm{H}), 6.06(\mathrm{~d}, J=7.3 \mathrm{~Hz}, 1 \mathrm{H}), 3.75-3.68(\mathrm{~m}, 1 \mathrm{H}), 2.40-2.32(\mathrm{~m}, 1 \mathrm{H}), 2.07-2.03(\mathrm{~m}$, 1H). ${ }^{13} \mathrm{C}$ NMR $\left(125 \mathrm{MHz}, \mathrm{CDCl}_{3}\right) \delta 151.9,133.2,133.2,132.5,130.2,129.9,128.9$, $128.3,123.4,121.4,121.3,121.1,118.0,103.8,72.8,27.1,26.1 .[\alpha]_{D}^{20}+136.52(c=$ 1.17 in $\mathrm{CHCl}_{3}$ ). ESI-HRMS: $[\mathrm{M}+\mathrm{H}]^{+}$calcd. For $\mathrm{C}_{17} \mathrm{H}_{13} \mathrm{ClNO}_{3} \mathrm{~S}^{+} \mathrm{m} / \mathrm{z}$ : 346.0299; found: 346.0302. The enantiomeric excess was determined by HPLC analysis on Daicel Chiralpak IB column ( $n$-hexane $/$ - $\mathrm{PrOH} /$ dichloromethane $=70 / 27 / 3,1 \mathrm{~mL} / \mathrm{min}$ ), $\lambda=$ $216 \mathrm{~nm}, t_{\text {major }}=9.59 \mathrm{~min}, t_{\text {minor }}=10.79 \mathrm{~min}, \mathrm{ee}=93 \%$.

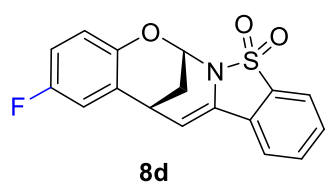

8d was synthesized following the general method ( $24 \mathrm{mg}, 73 \%$ yield) as a white solid. ${ }^{1} \mathrm{H}$ NMR $\left(500 \mathrm{MHz} \mathrm{CDCl}_{3}\right) \delta 7.80(\mathrm{~d}, J=7.8 \mathrm{~Hz}, 1 \mathrm{H}), 7.63-7.57(\mathrm{~m}, 2 \mathrm{H}), 7.57-7.50$ (m, 1H), $6.95-6.89(\mathrm{~m}, 1 \mathrm{H}), 6.81(\mathrm{dd}, J=13.4,5.6 \mathrm{~Hz}, 2 \mathrm{H}), 6.36(\mathrm{~d}, J=1.2 \mathrm{~Hz}, 1 \mathrm{H})$, $6.06(\mathrm{~d}, J=7.3 \mathrm{~Hz}, 1 \mathrm{H}), 3.73-3.65(\mathrm{~m}, 1 \mathrm{H}), 2.37(\mathrm{ddd}, J=12.9,2.6,1.9 \mathrm{~Hz}, 1 \mathrm{H}), 2.09$ - $1.99(\mathrm{~m}, 1 \mathrm{H}) .{ }^{13} \mathrm{C} \mathrm{NMR}\left(125 \mathrm{MHz}, \mathrm{CDCl}_{3}\right) \delta 157.0(\mathrm{~d}, J=240.0 \mathrm{~Hz}), 147.1,133.2$, $132.5,130.3,130.2,128.3,125.8$ (d, $J=7.2 \mathrm{~Hz}), 121.3,121.1,118.7(\mathrm{~d}, J=8.1 \mathrm{~Hz}$ ), $114.8(d, J=23.2 \mathrm{~Hz}), 114.2(\mathrm{~d}, J=23.2 \mathrm{~Hz}), 103.6,72.8,27.7,26.0 .[\alpha]_{D}^{20}+127.61(c$ $=1.92$ in $\mathrm{CHCl}_{3}$ ). ESI-HRMS: $[\mathrm{M}+\mathrm{H}]^{+}$calcd. For $\mathrm{C}_{17} \mathrm{H}_{13} \mathrm{FNO}_{3} \mathrm{~S}^{+} \mathrm{m} / \mathrm{z}$ : 330.0595; found: 330.0591. The enantiomeric excess was determined by HPLC analysis on Daicel Chiralpak IB column ( $n$-hexane/ $i$-PrOH/dichloromethane $=75 / 22 / 3,1 \mathrm{~mL} / \mathrm{min}$ ), $\lambda=$ $216 \mathrm{~nm}, t_{\text {major }}=11.07 \mathrm{~min}, t_{\text {minor }}=14.05 \mathrm{~min}$, ee $=95 \%$. 


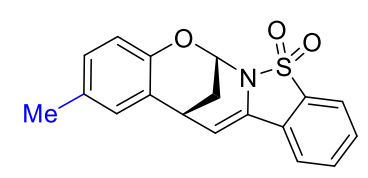

$8 e$

8e was synthesized following the general method ( $28 \mathrm{mg}, 86 \%$ yield) as a white solid. ${ }^{1} \mathrm{H}$ NMR $\left(500 \mathrm{MHz}, \mathrm{CDCl}_{3}\right) \delta 7.78(\mathrm{~d}, J=7.8 \mathrm{~Hz}, 1 \mathrm{H}), 7.60-7.53(\mathrm{~m}, 2 \mathrm{H}), 7.50(\mathrm{ddd}, J=$ 8.2, 6.4, $2.0 \mathrm{~Hz}, 1 \mathrm{H}), 6.95-6.86(\mathrm{~m}, 3 \mathrm{H}), 6.37-6.35(\mathrm{~m}, 1 \mathrm{H}), 6.08(\mathrm{~d}, J=7.0 \mathrm{~Hz}, 1 \mathrm{H})$, $3.72-3.63(m, 1 H), 2.45-2.33(m, 1 H), 2.26(s, 4 H), 2.04-2.01(m, 1 H) .{ }^{13} C N M R$ $\left(125 \mathrm{MHz} \mathrm{CDCl}_{3}\right) \delta 148.9,133.0,132.5,130.5,130.0,129.9,128.8,128.5,124.4$, $121.2,121.0,117.5,104.4,72.8,27.6,26.4,20.5 .[\alpha]_{D}^{20}+192.07\left(c=2.25\right.$ in $\left.\mathrm{CHCl}_{3}\right)$. ESI-HRMS: $[\mathrm{M}+\mathrm{H}]^{+}$calcd. For $\mathrm{C}_{18} \mathrm{H}_{16} \mathrm{NO}_{3} \mathrm{~S}^{+} \mathrm{m} / \mathrm{z}$ : 326.0845; found: 326.0848 . The enantiomeric excess was determined by HPLC analysis on Daicel Chiralpak IB column ( $n$-hexane $/ \mathrm{i}-\mathrm{PrOH} /$ dichloromethane $=75 / 22 / 3,1 \mathrm{~mL} / \mathrm{min}$ ), $\lambda=216 \mathrm{~nm}, t_{\text {major }}=9.35$ $\min , t_{\text {minor }}=10.77 \min$, ee $=95 \%$.

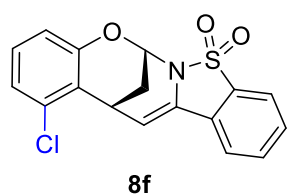

$8 f$ was synthesized following the general method ( $25 \mathrm{mg}, 72 \%$ yield) as a white solid. ${ }^{1} \mathrm{H}$ NMR $\left(500 \mathrm{MHz} \mathrm{CDCl}_{3}\right) \delta 7.80(\mathrm{~d}, J=7.8 \mathrm{~Hz}, 1 \mathrm{H}), 7.66-7.57(\mathrm{~m}, 2 \mathrm{H}), 7.56-7.47$ (m, 1H), $7.05(\mathrm{t}, J=8.1 \mathrm{~Hz}, 1 \mathrm{H}), 6.97(\mathrm{dd}, J=7.8,0.8 \mathrm{~Hz}, 1 \mathrm{H}), 6.92(\mathrm{~d}, J=8.2 \mathrm{~Hz}, 1 \mathrm{H})$, $6.39(\mathrm{~d}, J=1.3 \mathrm{~Hz}, 1 \mathrm{H}), 6.21(\mathrm{~d}, J=7.3 \mathrm{~Hz}, 1 \mathrm{H}), 4.21-4.08(\mathrm{~m}, 1 \mathrm{H}), 2.34(\mathrm{dt}, J=13.0$, $2.3 \mathrm{~Hz}, 1 \mathrm{H}), 2.07(\mathrm{dt}, J=13.0,2.5 \mathrm{~Hz}, 1 \mathrm{H}) .{ }^{13} \mathrm{C} \mathrm{NMR}\left(125 \mathrm{MHz}, \mathrm{CDCl}_{3}\right) \delta$ 152.6, 133.4, $132.9,132.7,131.1,130.4,128.6,128.4,123.6,122.0,121.5,121.4,116.6,102.8$, 73.0, 26.3, 25.1. $[\alpha]_{D}^{20}+308.29\left(c=0.38\right.$ in $\mathrm{CHCl}_{3}$ ). ESI-HRMS: $[\mathrm{M}+\mathrm{H}]^{+}$calcd. For $\mathrm{C}_{17} \mathrm{H}_{13} \mathrm{ClNO}_{3} \mathrm{~S}^{+} \mathrm{m} / \mathrm{z}$ : 346.0299; found: 346.0301. The enantiomeric excess was determined by HPLC analysis on Daicel Chiralpak IB column ( $n$-hexane/i-PrOH $=$ $70 / 30,1 \mathrm{~mL} / \mathrm{min}), \lambda=210 \mathrm{~nm}, t_{\text {major }}=8.66 \mathrm{~min}, t_{\text {minor }}=10.09 \mathrm{~min}$, ee $=99 \%$. 


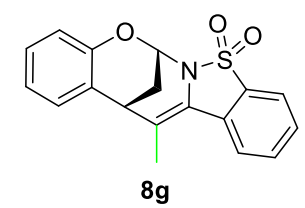

$8 \mathrm{~g}$ was synthesized following the general method ( $23 \mathrm{mg}, 71 \%$ yield) as a white solid. ${ }^{1} \mathrm{H}$ NMR $\left(500 \mathrm{MHz}, \mathrm{CDCl}_{3}\right) \delta 7.84(\mathrm{~d}, J=7.8 \mathrm{~Hz}, 1 \mathrm{H}), 7.77(\mathrm{~d}, J=8.1 \mathrm{~Hz}, 1 \mathrm{H}), 7.63-$ $7.56(\mathrm{~m}, 1 \mathrm{H}), 7.49(\mathrm{t}, J=7.6 \mathrm{~Hz}, 1 \mathrm{H}), 7.20-7.11(\mathrm{~m}, 2 \mathrm{H}), 6.99(\mathrm{~d}, J=8.1 \mathrm{~Hz}, 1 \mathrm{H}), 6.89$ (dd, $J=10.7,4.1 \mathrm{~Hz}, 1 \mathrm{H}), 6.35(\mathrm{~d}, J=1.3 \mathrm{~Hz}, 1 \mathrm{H}), 3.44(\mathrm{~s}, 1 \mathrm{H}), 2.40-2.31(\mathrm{~m}, 4 \mathrm{H})$, $2.08(\mathrm{dt}, J=12.8,2.6 \mathrm{~Hz}, 1 \mathrm{H}) .{ }^{13} \mathrm{C}$ NMR $\left(125 \mathrm{MHz}, \mathrm{CDCl}_{3}\right) \delta 151.5,133.1,129.7,129.1$, 128.6, 127.7, 124.6, 124.4, 124.0, 121.8, 121.2, 118.5, 117.7, 72.6, 36.6, 26.1, 19.0. $[\alpha]_{D}^{20}+115.21\left(c=1.92\right.$ in $\left.\mathrm{CHCl}_{3}\right)$. ESI-HRMS: $[\mathrm{M}+\mathrm{H}]^{+}$calcd. For $\mathrm{C}_{18} \mathrm{H}_{16} \mathrm{NO}_{3} \mathrm{~S}^{+} \mathrm{m} / \mathrm{z}$ : 326.0845; found: 326.0844 . The enantiomeric excess was determined by HPLC analysis on Daicel Chiralpak IB column ( $n$-hexane/i-PrOH/dichloromethane $=70 / 27 / 3$, $1 \mathrm{~mL} / \mathrm{min}), \lambda=210 \mathrm{~nm}, t_{\text {major }}=7.97 \mathrm{~min}, t_{\text {minor }}=10.20 \mathrm{~min}$, ee $=98 \%$.

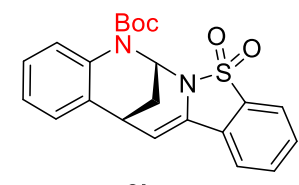

$8 \mathrm{~h}$

$8 \mathrm{~h}$ was synthesized following the general method ( $27 \mathrm{mg}, 66 \%$ yield) as a white solid. ${ }^{1} \mathrm{H}$ NMR $\left(500 \mathrm{MHz}^{\left.\mathrm{C} C \mathrm{CDC}_{3}\right)} \delta 8.46(\mathrm{~d}, J=8.5 \mathrm{~Hz}, 1 \mathrm{H}), 7.75(\mathrm{~d}, J=7.8 \mathrm{~Hz}, 1 \mathrm{H}), 7.58-\right.$ $7.42(\mathrm{~m}, 3 \mathrm{H}), 7.18-7.14(\mathrm{~m}, 2 \mathrm{H}), 7.04$ (brs, 1H), $7.01-6.94(\mathrm{~m}, 1 \mathrm{H}), 6.04$ (d, J = 7.6 $\mathrm{Hz}, 1 \mathrm{H}), 3.84-3.70(\mathrm{~m}, 1 \mathrm{H}), 2.40-2.36(\mathrm{~m}, 1 \mathrm{H}), 2.03(\mathrm{ddd}, J=12.6,3.6,2.4 \mathrm{~Hz}, 1 \mathrm{H})$, 1.68 (s, 9H). ${ }^{13} \mathrm{C}$ NMR $\left(125 \mathrm{MHz}, \mathrm{CDCl}_{3}\right) \delta 151.7,135.4,132.8,132.6,130.3,129.9$, $128.6,128.5,128.0,127.2,123.0,122.3,121.0,120.8,104.5,83.1,57.4,30.1,28.2$, 26.4. $[\alpha]_{D}^{20}-15.08\left(c=1.08\right.$ in $\mathrm{CHCl}_{3}$ ). ESI-HRMS: $[\mathrm{M}+\mathrm{H}]^{+}$calcd. For $\mathrm{C}_{22} \mathrm{H}_{23} \mathrm{~N}_{2} \mathrm{O}_{4} \mathrm{~S}^{+} \mathrm{m} / \mathrm{z}$ : 411.1373; found: 411.1375. The enantiomeric excess was determined by HPLC analysis on Daicel Chiralpak IA column ( $n$-hexane/i-PrOH/dichloromethane $=70 / 27 / 3$, $1 \mathrm{~mL} / \mathrm{min}), \lambda=225 \mathrm{~nm}, t_{\text {major }}=8.35 \mathrm{~min}, t_{\text {minor }}=13.34 \mathrm{~min}$, ee $=92 \%$. 


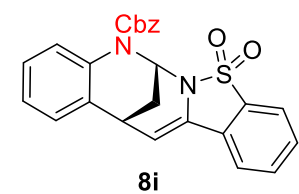

$\mathbf{8 i}$ was synthesized following the general method ( $30 \mathrm{mg}, 68 \%$ yield) as a white solid. ${ }^{1} \mathrm{H}$ NMR $\left(500 \mathrm{MHz}, \mathrm{CDCl}_{3}\right) \delta 8.39(\mathrm{~d}, J=8.4 \mathrm{~Hz}, 1 \mathrm{H}), 7.77(\mathrm{~d}, J=7.7 \mathrm{~Hz}, 1 \mathrm{H}), 7.65-$ $7.47(\mathrm{~m}, 5 \mathrm{H}), 7.43(\mathrm{t}, J=7.5 \mathrm{~Hz}, 2 \mathrm{H}), 7.36(\mathrm{t}, J=7.2 \mathrm{~Hz}, 1 \mathrm{H}), 7.17(\mathrm{t}, J=8.2 \mathrm{~Hz}, 2 \mathrm{H})$, 7.09 (s, 1H), 7.01 (t, J = 7.4 Hz, 1H), 6.04 (d, J = 7.6 Hz, 1H), 5.53 (d, J = $12.1 \mathrm{~Hz}, 1 \mathrm{H}$ ), $5.31(\mathrm{~d}, J=12.1 \mathrm{~Hz}, 1 \mathrm{H}), 3.75(\mathrm{~d}, J=7.4 \mathrm{~Hz}, 1 \mathrm{H}), 2.33(\mathrm{~d}, J=12.7 \mathrm{~Hz}, 1 \mathrm{H}), 2.11-2.01$ (m, 1H). ${ }^{13} \mathrm{C} N M R\left(125 \mathrm{MHz}, \mathrm{CDCl}_{3}\right) \delta 153.0,135.8,135.0,132.9,132.5,130.1,129.9$, $128.8,128.6,128.5,128.4,128.4,128.3,127.4,123.3,122.1,121.1,120.9,104.2$, 68.4, 57.6, 30.0, 26.4. $[\alpha]_{D}{ }^{20}-15.85\left(c=1.42\right.$ in $\left.\mathrm{CHCl}_{3}\right)$. ESI-HRMS: $[\mathrm{M}+\mathrm{H}]^{+}$calcd. For $\mathrm{C}_{25} \mathrm{H}_{21} \mathrm{~N}_{2} \mathrm{O}_{4} \mathrm{~S}^{+} \mathrm{m} / \mathrm{z}:$ 445.1217; found: 445.1213. The enantiomeric excess was determined by HPLC analysis on Daicel Chiralpak IA column ( $n$-hexane $/ \mathrm{i}$-PrOH/dichloromethane $=70 / 27 / 3,1 \mathrm{~mL} / \mathrm{min}$ ), $\lambda=225 \mathrm{~nm}, t_{\text {major }}=18.44$ $\min , t_{\text {minor }}=38.51 \mathrm{~min}$, ee $=85 \%$.

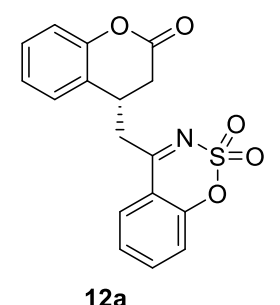

12a was synthesized following the general method (18 $\mathrm{mg}, 53 \%$ yield) as a white solid. ${ }^{1} \mathrm{H}$ NMR $\left(500 \mathrm{MHz}, \mathrm{CDCl}_{3}\right) \delta 7.74-7.66(\mathrm{~m}, 1 \mathrm{H}), 7.58(\mathrm{~d}, J=8.0 \mathrm{~Hz}, 1 \mathrm{H}), 7.35-$ $7.24(\mathrm{~m}, 4 \mathrm{H}), 7.10(\mathrm{t}, J=7.3 \mathrm{~Hz}, 2 \mathrm{H}), 4.01-3.90(\mathrm{~m}, 1 \mathrm{H}), 3.29$ (ddd, $J=39.6,17.1,7.2$ $\mathrm{Hz}, 2 \mathrm{H}), 3.04-2.92(\mathrm{~m}, 2 \mathrm{H}) .{ }^{13} \mathrm{C} N M R\left(125 \mathrm{MHz}, \mathrm{CDCl}_{3}\right) \delta 176.5,167.3,153.6,151.4$, $137.4,129.3,128.1,127.6,126.0,125.0,124.5,119.3,117.4,116.1,39.5,34.6,31.8$. $[\alpha]_{D}{ }^{20}-62.70\left(c=1.75\right.$ in $\left.\mathrm{CHCl}_{3}\right)$. ESI-HRMS: $[\mathrm{M}+\mathrm{H}]^{+}$calcd. For $\mathrm{C}_{17} \mathrm{H}_{14} \mathrm{NO}_{5} \mathrm{~S}^{+} \mathrm{m} / \mathrm{z}$ : 344.0587; found: 344.0589. The enantiomeric excess was determined by HPLC analysis on Daicel Chiralpak IC column ( $n$-hexane/ $i-\mathrm{PrOH} /$ dichloromethane $=60 / 37 / 3$, $1 \mathrm{~mL} / \mathrm{min}), \lambda=270 \mathrm{~nm}, t_{\text {major }}=39.43 \mathrm{~min}, t_{\text {minor }}=22.96 \mathrm{~min}$, ee $=96 \%$. 


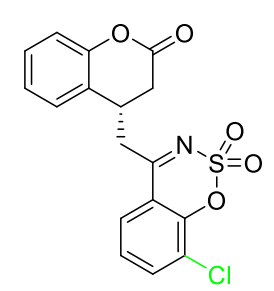

$12 b$

12b was synthesized following the general method (23 $\mathrm{mg}, 61 \%$ yield) as a white solid. ${ }^{1} \mathrm{H}$ NMR $\left(500 \mathrm{MHz}, \mathrm{CDCl}_{3}\right.$ ) $\delta 7.74$ (dd, $\left.J=8.0,1.2 \mathrm{~Hz}, 1 \mathrm{H}\right), 7.50$ (dd, $J=8.0,1.1$ $\mathrm{Hz}, 1 \mathrm{H}), 7.31-7.22(\mathrm{~m}, 3 \mathrm{H}), 7.10(\mathrm{dd}, J=12.3,7.8 \mathrm{~Hz}, 2 \mathrm{H}), 3.99-3.90(\mathrm{~m}, 1 \mathrm{H}), 3.33$ (dd, $J=17.3,7.0 \mathrm{~Hz}, 1 \mathrm{H}$ ), $3.26(\mathrm{dd}, J=17.2,7.4 \mathrm{~Hz}, 1 \mathrm{H}), 2.99(\mathrm{~d}, J=4.4 \mathrm{~Hz}, 2 \mathrm{H}) .{ }^{13} \mathrm{C}$ NMR $\left(125 \mathrm{MHz}, \mathrm{CDCl}_{3}\right) \delta$ 176.6, 167.5, 151.5, 149.8, 137.8, 129.6, 128.3, 126.1, 126.1, $125.3,124.9,124.5,117.7,117.6,39.9,34.8,32.0 .[\alpha]_{\mathrm{D}}{ }^{20}-82.57\left(c=1.58\right.$ in $\mathrm{CHCl}_{3}$ ). ESI-HRMS: $[\mathrm{M}+\mathrm{H}]^{+}$calcd. For $\mathrm{C}_{17} \mathrm{H}_{13} \mathrm{ClNO}_{5} \mathrm{~S}^{+} \mathrm{m} / \mathrm{z}$ : 378.0197; found: 378.0200 . The enantiomeric excess was determined by HPLC analysis on Daicel Chiralpak IC column ( $n$-hexane $/ \mathrm{i}-\mathrm{PrOH} /$ dichloromethane $=45 / 5 / 50,1 \mathrm{~mL} / \mathrm{min}$ ), $\lambda=260 \mathrm{~nm}, t_{\text {major }}=10.12$ $\min , t_{\text {minor }}=6.91 \mathrm{~min}$, ee $=90 \%$.

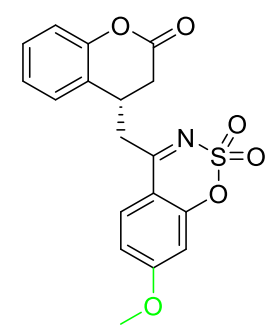

12c

12c was synthesized following the general method ( $24 \mathrm{mg}, 64 \%$ yield) as a white solid. ${ }^{1} \mathrm{H}$ NMR $\left(500 \mathrm{MHz}, \mathrm{CDCl}_{3}\right) \delta 7.46(\mathrm{~d}, J=9.0 \mathrm{~Hz}, 1 \mathrm{H}), 7.31-7.24(\mathrm{~m}, 2 \mathrm{H}), 7.12-$ $7.07(\mathrm{~m}, 2 \mathrm{H}), 6.78(\mathrm{dd}, J=9.0,2.4 \mathrm{~Hz}, 1 \mathrm{H}), 6.73(\mathrm{~d}, J=2.4 \mathrm{~Hz}, 1 \mathrm{H}), 3.99-3.86(\mathrm{~m}, 4 \mathrm{H})$, $3.24(\mathrm{dd}, J=16.6,6.9 \mathrm{~Hz}, 1 \mathrm{H}), 3.16(\mathrm{dd}, J=16.6,7.6 \mathrm{~Hz}, 1 \mathrm{H}), 2.97(\mathrm{~d}, J=4.4 \mathrm{~Hz}, 2 \mathrm{H})$. ${ }^{13} \mathrm{C}$ NMR $\left(125 \mathrm{MHz}, \mathrm{CDCl}_{3}\right) \delta 176.1,167.6,167.0,156.3,151.5,129.6,129.4,128.3$, $125.2,124.8,117.5,113.7,109.8,103.4,56.6,39.6,34.8,32.3 .[\alpha]_{D}^{20}-103.60(c=$ 0.75 in $\mathrm{CHCl}_{3}$ ). ESI-HRMS: $[\mathrm{M}+\mathrm{H}]^{+}$calcd. For $\mathrm{C}_{18} \mathrm{H}_{16} \mathrm{NO}_{6} \mathrm{~S}^{+} \mathrm{m} / \mathrm{z}$ : 374.0693; found: 374.0698. The enantiomeric excess was determined by HPLC analysis on Daicel Chiralpak IC column ( $n$-hexane $/ \mathrm{i}$-PrOH/dichloromethane $=40 / 10 / 50,1 \mathrm{~mL} / \mathrm{min}$ ), $\lambda=$ $299 \mathrm{~nm}, t_{\text {major }}=10.59 \mathrm{~min}, t_{\text {minor }}=7.01 \mathrm{~min}$, ee $=96 \%$. 


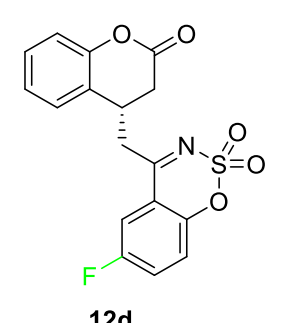

$12 d$

12d was synthesized following the general method (17 $\mathrm{mg}, 47 \%$ yield) as a white solid. ${ }^{1} \mathrm{H}$ NMR $\left(500 \mathrm{MHz} \mathrm{CDCl}_{3}\right) \delta 7.45-7.38(\mathrm{~m}, 1 \mathrm{H}), 7.35-7.22(\mathrm{~m}, 4 \mathrm{H}), 7.11(\mathrm{t}, J=$ $7.1 \mathrm{~Hz}, 2 \mathrm{H}), 3.99-3.89(\mathrm{~m}, 1 \mathrm{H}), 3.31-3.19(\mathrm{~m}, 2 \mathrm{H}), 3.07-2.93(\mathrm{~m}, 2 \mathrm{H}) .{ }^{13} \mathrm{C} N M R$ $\left(125 \mathrm{MHz}, \mathrm{CDCl}_{3}\right) \delta 175.6,167.2,159.0(\mathrm{~d}, J=249.4 \mathrm{~Hz}), 151.4,149.5(\mathrm{~d}, J=2.3 \mathrm{~Hz})$, 129.4, 128.0, 125.1, 124.6 (d, $J=24.0 \mathrm{~Hz}), 124.3,121.1$ (d, $J=8.0 \mathrm{~Hz}$ ), 117.5, 116.8 (d) $J=7.7 \mathrm{~Hz}), 113.7(\mathrm{~d}, J=25.3 \mathrm{~Hz}), 39.6,34.5,31.7 .[\alpha]_{\mathrm{D}}{ }^{20}-32.70\left(c=1.00\right.$ in $\left.\mathrm{CHCl}_{3}\right)$. ESI-HRMS: $[\mathrm{M}+\mathrm{H}]^{+}$calcd. For $\mathrm{C}_{17} \mathrm{H}_{13} \mathrm{FNO}_{5} \mathrm{~S}^{+} \mathrm{m} / \mathrm{z}$ : 362.0493; found: 362.0495. The enantiomeric excess was determined by HPLC analysis on Daicel Chiralpak IC column ( $n$-hexane $/$ i-PrOH/dichloromethane $=60 / 37 / 3,1 \mathrm{~mL} / \mathrm{min}$ ), $\lambda=260 \mathrm{~nm}, t_{\text {major }}=28.29$ $\min , t_{\text {minor }}=16.79 \mathrm{~min}$, ee $=85 \%$.

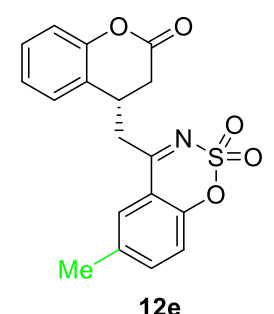

12e was synthesized following the general method (20 mg, 56\% yield) as a white solid. ${ }^{1} \mathrm{H}$ NMR $\left(500 \mathrm{MHz}, \mathrm{CDCl}_{3}\right) \delta 7.48(\mathrm{~d}, J=8.2 \mathrm{~Hz}, 1 \mathrm{H}), 7.33(\mathrm{~s}, 1 \mathrm{H}), 7.28$ (dd, $J=$ 14.1, 6.0 Hz, 2H), $7.17(\mathrm{~d}, J=8.4 \mathrm{~Hz}, 1 \mathrm{H}), 7.13-7.05(\mathrm{~m}, 2 \mathrm{H}), 4.03-3.88(\mathrm{~m}, 1 \mathrm{H})$, $3.33-3.20(\mathrm{~m}, 2 \mathrm{H}), 3.05-2.91(\mathrm{~m}, 2 \mathrm{H}), 2.35(\mathrm{~s}, 3 \mathrm{H}) .{ }^{13} \mathrm{C} \mathrm{NMR}\left(125 \mathrm{MHz}, \mathrm{CDCl}_{3}\right) \delta$ $176.6,167.4,151.5,151.4,138.2,136.2,129.3,128.2,127.6,125.0,124.6,118.9$, 117.3, 115.9, 39.5, 34.6, 31.8, 20.8. $[\alpha]_{D}^{20}-81.91\left(c=0.58\right.$ in $\mathrm{CHCl}_{3}$ ). ESI-HRMS: $[\mathrm{M}+\mathrm{H}]^{+}$calcd. For $\mathrm{C}_{18} \mathrm{H}_{16} \mathrm{NO}_{5} \mathrm{~S}^{+} \mathrm{m} / \mathrm{z}$ : 358.0744; found: 358.0745. The enantiomeric excess was determined by HPLC analysis on Daicel Chiralpak IC column ( $n$-hexane $/ \mathrm{i}$-PrOH/dichloromethane $=45 / 5 / 50,1 \mathrm{~mL} / \mathrm{min}$ ), $\lambda=260 \mathrm{~nm}, t_{\text {major }}=12.35$ $\min , t_{\text {minor }}=7.93 \mathrm{~min}$, ee $=94 \%$. 


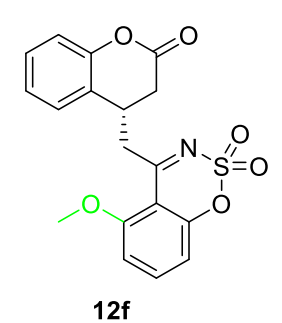

12e was synthesized following the general method ( $23 \mathrm{mg}, 62 \%$ yield) as a white solid. ${ }^{1} \mathrm{H}$ NMR $\left(500 \mathrm{MHz}, \mathrm{CDCl}_{3}\right) \delta 7.58(\mathrm{t}, J=8.4 \mathrm{~Hz}, 1 \mathrm{H}), 7.29-7.20(\mathrm{~m}, 2 \mathrm{H}), 7.11-$ $7.03(\mathrm{~m}, 2 \mathrm{H}), 6.87(\mathrm{~d}, J=8.3 \mathrm{~Hz}, 1 \mathrm{H}), 6.79(\mathrm{~d}, J=8.6 \mathrm{~Hz}, 1 \mathrm{H}), 3.92-3.83(\mathrm{~m}, 4 \mathrm{H}), 3.53$ (dd, $J=16.8,7.1 \mathrm{~Hz}, 1 \mathrm{H}), 3.36(\mathrm{dd}, J=16.8,7.6 \mathrm{~Hz}, 1 \mathrm{H}), 2.91(\mathrm{~d}, J=4.5 \mathrm{~Hz}, 2 \mathrm{H}) .{ }^{13} \mathrm{C}$ $\operatorname{NMR}\left(125 \mathrm{MHz}, \mathrm{CDCl}_{3}\right) \delta 178.3,167.9,159.5,154.6,151.7,137.7,129.1,128.3,125.2$, $125.0,117.3,111.4,109.0,108.4,56.7,43.9,34.8,32.6 .[\alpha]_{D}^{20}-59.59$ (c $=1.83$ in $\mathrm{CHCl}_{3}$ ). ESI-HRMS: $[\mathrm{M}+\mathrm{H}]^{+}$calcd. For $\mathrm{C}_{18} \mathrm{H}_{16} \mathrm{NO}_{6} \mathrm{~S}^{+} \mathrm{m} / \mathrm{z}$ : 374.0693; found: 374.0690 . The enantiomeric excess was determined by HPLC analysis on Daicel Chiralpak IC column ( $n$-hexane/i-PrOH/dichloromethane $=40 / 10 / 50,1 \mathrm{~mL} / \mathrm{min}$ ), $\lambda=299 \mathrm{~nm}, t_{\text {major }}$ $=7.29 \mathrm{~min}, t_{\text {minor }}=5.91 \mathrm{~min}, \mathrm{ee}=91 \%$.

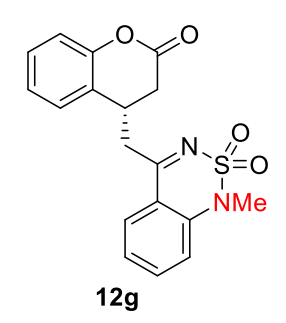

$12 \mathrm{~g}$ was synthesized following the general method ( $24 \mathrm{mg}, 67 \%$ yield) as a white solid. ${ }^{1} \mathrm{H}$ NMR $\left(500 \mathrm{MHz}, \mathrm{CDCl}_{3}\right) \delta 7.67(\mathrm{t}, J=7.9 \mathrm{~Hz}, 1 \mathrm{H}), 7.59(\mathrm{~d}, J=8.1 \mathrm{~Hz}, 1 \mathrm{H}), 7.32$ $-7.24(\mathrm{~m}, 2 \mathrm{H}), 7.19-7.05(\mathrm{~m}, 4 \mathrm{H}), 3.97(\mathrm{~s}, 1 \mathrm{H}), 3.56(\mathrm{~s}, 3 \mathrm{H}), 3.27$ (ddd, J = 24.8, 16.8, $7.2 \mathrm{~Hz}, 2 \mathrm{H}), 3.06-2.88(\mathrm{~m}, 2 \mathrm{H}) .{ }^{13} \mathrm{C} N M R\left(125 \mathrm{MHz}, \mathrm{CDCl}_{3}\right) \delta 173.6,167.7,151.4$, $143.5,136.3,129.1,128.2,128.1,125.0,124.9,122.2,117.6,117.2,115.4,39.8,34.5$, 32.1, 31.3. $[\alpha]_{D}{ }^{20}-59.97\left(c=1.83\right.$ in $\mathrm{CHCl}_{3}$ ). ESI-HRMS: $[\mathrm{M}+\mathrm{H}]^{+}$calcd. For $\mathrm{C}_{18} \mathrm{H}_{17} \mathrm{~N}_{2} \mathrm{O}_{4} \mathrm{~S}^{+}$ m/z: 357.0904; found: 357.0900. The enantiomeric excess was determined by HPLC analysis on Daicel Chiralpak IC column ( $n$-hexane/i-PrOH/dichloromethane = $40 / 10 / 50,1 \mathrm{~mL} / \mathrm{min}), \lambda=225 \mathrm{~nm}, t_{\text {major }}=14.53 \mathrm{~min}, t_{\text {minor }}=9.04 \mathrm{~min}$, ee $=93 \%$. 


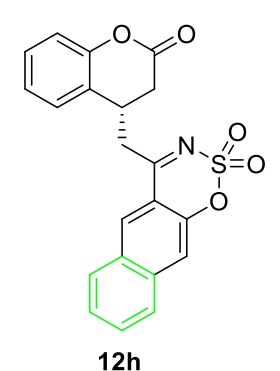

$12 \mathrm{~h}$ was synthesized following the general method (20 mg, 51\% yield) as a white solid. ${ }^{1} \mathrm{H}$ NMR $\left(500 \mathrm{MHz}, \mathrm{CDCl}_{3}\right) \delta 8.41(\mathrm{~d}, J=8.4 \mathrm{~Hz}, 1 \mathrm{H}), 7.88(\mathrm{~d}, J=8.1 \mathrm{~Hz}, 1 \mathrm{H}), 7.76$ (t, $J=7.3 \mathrm{~Hz}, 1 \mathrm{H}), 7.70(\mathrm{t}, J=7.5 \mathrm{~Hz}, 1 \mathrm{H}), 7.66(\mathrm{~d}, J=8.8 \mathrm{~Hz}, 1 \mathrm{H}), 7.46(\mathrm{~d}, J=8.8 \mathrm{~Hz}$, 1H), $7.33-7.24(\mathrm{~m}, 2 \mathrm{H}), 7.11-7.08(\mathrm{~m}, 2 \mathrm{H}), 4.06-3.93(\mathrm{~m}, 1 \mathrm{H}), 3.37$ (ddd, $J=45.0$, 17.1, 7.2 Hz, 2H), $3.11-2.93(\mathrm{~m}, 2 \mathrm{H}) .{ }^{13} \mathrm{C} \mathrm{NMR}\left(125 \mathrm{MHz}, \mathrm{CDCl}_{3}\right) \delta 177.3,167.4$, 152.6 , 151.4, 137.4, 131.5, 129.3, 128.4, 128.1, 128.0, 125.5, 125.0, 124.6, 123.4, $122.9,120.6,117.4,111.4,40.1,34.6,31.9$. $[\alpha]_{\mathrm{D}}^{20}-112.78$ ( $c=1.58$ in $\mathrm{CHCl}_{3}$ ). ESI-HRMS: $[\mathrm{M}+\mathrm{H}]^{+}$calcd. For $\mathrm{C}_{21} \mathrm{H}_{16} \mathrm{NO}_{5} \mathrm{~S}^{+} \mathrm{m} / \mathrm{z}$ : 394.0744; found: 394.0746. The enantiomeric excess was determined by HPLC analysis on Daicel Chiralpak IC column ( $n$-hexane $/ i-\mathrm{PrOH} /$ dichloromethane $=45 / 5 / 50,1 \mathrm{~mL} / \mathrm{min}$ ), $\lambda=260 \mathrm{~nm}, t_{\text {major }}=11.85$ $\min , t_{\text {minor }}=7.93 \mathrm{~min}$, ee $=95 \%$.

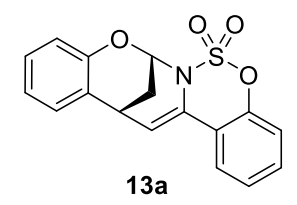

13a was synthesized following the general method (18 $\mathrm{mg}, 55 \%$ yield) as a white solid. ${ }^{1} \mathrm{H}$ NMR $\left(500 \mathrm{MHz}, \mathrm{CDCl}_{3}\right) \delta 7.54(\mathrm{dd}, J=8.1,1.0 \mathrm{~Hz}, 1 \mathrm{H}), 7.35-7.28(\mathrm{~m}, 1 \mathrm{H})$, $7.20(\mathrm{t}, J=7.4 \mathrm{~Hz}, 1 \mathrm{H}), 7.17-7.06(\mathrm{~m}, 3 \mathrm{H}), 7.00(\mathrm{~d}, J=8.0 \mathrm{~Hz}, 1 \mathrm{H}), 6.91(\mathrm{t}, J=7.4 \mathrm{~Hz}$, $1 \mathrm{H}), 6.46(\mathrm{~d}, J=1.6 \mathrm{~Hz}, 1 \mathrm{H}), 6.19(\mathrm{~d}, J=7.5 \mathrm{~Hz}, 1 \mathrm{H}), 3.73-3.62(\mathrm{~m}, 1 \mathrm{H}), 2.33(\mathrm{dd}, J=$ 13.0, $0.7 \mathrm{~Hz}, 1 \mathrm{H}), 2.14-2.10(\mathrm{~m}, 1 \mathrm{H}) .{ }^{13} \mathrm{C} \mathrm{NMR}\left(125 \mathrm{MHz}, \mathrm{CDCl}_{3}\right) \delta 151.0,148.2$, $131.0,130.5,128.3,127.9,126.2,124.9,124.0,121.4,119.3,118.8,117.4,108.9$, 76.9, 27.4, 25.6. $[\alpha]_{D}^{20}-16.47\left(c=1.42\right.$ in $\left.\mathrm{CHCl}_{3}\right)$. ESI-HRMS: $[\mathrm{M}+\mathrm{H}]^{+}$calcd. For $\mathrm{C}_{17} \mathrm{H}_{14} \mathrm{NO}_{4} \mathrm{~S}^{+} \mathrm{m} / \mathrm{z}$ : 328.0638; found: 328.0636. The enantiomeric excess was determined by HPLC analysis on Daicel Chiralpak IB column ( $n$-hexane/i-PrOH/dichloromethane $=80 / 17 / 3,1 \mathrm{~mL} / \mathrm{min}$ ), $\lambda=216 \mathrm{~nm}, t_{\text {major }}=8.24$ $\min , t_{\text {minor }}=12.06 \mathrm{~min}$, ee $=94 \%$. 


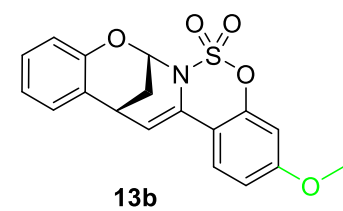

13b was synthesized following the general method ( $25 \mathrm{mg}, 70 \%$ yield) as a white solid. ${ }^{1} \mathrm{H}$ NMR $\left(500 \mathrm{MHz}, \mathrm{CDCl}_{3}\right) \delta 7.42(\mathrm{~d}, J=9.0 \mathrm{~Hz}, 1 \mathrm{H}), 7.15-7.09(\mathrm{~m}, 2 \mathrm{H}), 7.00(\mathrm{~d}$, $J=8.1 \mathrm{~Hz}, 1 \mathrm{H}), 6.90(\mathrm{t}, J=7.4 \mathrm{~Hz}, 1 \mathrm{H}), 6.76(\mathrm{dd}, J=8.9,2.4 \mathrm{~Hz}, 1 \mathrm{H}), 6.59(\mathrm{~d}, J=2.4 \mathrm{~Hz}$, 1H), $6.44(\mathrm{~s}, 1 \mathrm{H}), 6.01(\mathrm{~d}, J=7.6 \mathrm{~Hz}, 1 \mathrm{H}), 3.80(\mathrm{~s}, 3 \mathrm{H}), 3.70-3.60(\mathrm{~m}, 1 \mathrm{H}), 2.32(\mathrm{~d}, J=$ $12.9 \mathrm{~Hz}, 1 \mathrm{H}), 2.12$ (d, $J=13.0 \mathrm{~Hz}, 1 \mathrm{H}) .{ }^{13} \mathrm{C} \mathrm{NMR}\left(125 \mathrm{MHz}, \mathrm{CDCl}_{3}\right) \delta$ 161.2, 151.0, $149.0,130.8,128.2,127.8,125.3,125.0,121.3,117.4,113.6,111.2,106.7,103.5$, 76.9, 55.7, 27.3, 25.8. $[\alpha]_{\mathrm{D}}{ }^{20}-12.92\left(c=0.67\right.$ in $\left.\mathrm{CHCl}_{3}\right)$. ESI-HRMS: $[\mathrm{M}+\mathrm{H}]^{+}$calcd. For $\mathrm{C}_{18} \mathrm{H}_{16} \mathrm{NO}_{5} \mathrm{~S}^{+} \mathrm{m} / \mathrm{z}$ : 358.0744; found: 358.0748. The enantiomeric excess was determined by HPLC analysis on Daicel Chiralpak IB column ( $n$-hexane/i-PrOH = $80 / 20,1 \mathrm{~mL} / \mathrm{min}), \lambda=210 \mathrm{~nm}, t_{\text {major }}=11.09 \mathrm{~min}, t_{\text {minor }}=15.37 \mathrm{~min}$, ee $=98 \%$.

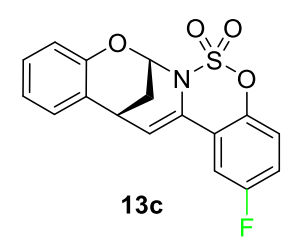

13c was synthesized following the general method (18 $\mathrm{mg}, 52 \%$ yield) as a white solid. ${ }^{1} \mathrm{H}$ NMR $\left(500 \mathrm{MHz}, \mathrm{CDCl}_{3}\right) \delta 7.22(\mathrm{dd}, J=9.2,2.7 \mathrm{~Hz}, 1 \mathrm{H}), 7.18-7.05(\mathrm{~m}, 3 \mathrm{H})$, $7.05-6.97(\mathrm{~m}, 2 \mathrm{H}), 6.91(\mathrm{t}, J=7.4 \mathrm{~Hz}, 1 \mathrm{H}), 6.45(\mathrm{~d}, J=1.7 \mathrm{~Hz}, 1 \mathrm{H}), 6.16(\mathrm{~d}, J=7.5 \mathrm{~Hz}$, $1 \mathrm{H}), 3.79-3.63(\mathrm{~m}, 1 \mathrm{H}), 2.34(\mathrm{~d}, J=13.0 \mathrm{~Hz}, 1 \mathrm{H}), 2.12(\mathrm{dt}, J=13.0,2.4 \mathrm{~Hz}, 1 \mathrm{H}) .{ }^{13} \mathrm{C}$ $\operatorname{NMR}\left(125 \mathrm{MHz}, \mathrm{CDCl}_{3}\right) \delta 160.2(\mathrm{~d}, J=245.4 \mathrm{~Hz}, 1 \mathrm{H}), 151.2,144.4,130.7$ (d, $J=3.0 \mathrm{~Hz}$, 1H), 128.6, 128.2, 124.8, 121.7, $121.2(\mathrm{~d}, J=8.8 \mathrm{~Hz}, 1 \mathrm{H}), 120.6$ (d, $J=8.6 \mathrm{~Hz}, 1 \mathrm{H})$, $117.9,117.7,110.7$ (d, $J=26.4 \mathrm{~Hz}, 1 \mathrm{H}), 110.4,77.1,27.6,25.7 .[\alpha]_{D}{ }^{20}-24.53(c=1.17$ in $\mathrm{CHCl}_{3}$ ). ESI-HRMS: $[\mathrm{M}+\mathrm{H}]^{+}$calcd. For $\mathrm{C}_{17} \mathrm{H}_{13} \mathrm{FNO}_{4} \mathrm{~S}^{+} \mathrm{m} / \mathrm{z}$ : 346.0544; found: 346.0547. The enantiomeric excess was determined by HPLC analysis on Daicel Chiralpak IB column ( $n$-hexane $/ \mathrm{i}-\mathrm{PrOH}=80 / 20,1 \mathrm{~mL} / \mathrm{min}$ ), $\lambda=210 \mathrm{~nm}, t_{\text {major }}=8.81 \mathrm{~min}, t_{\text {minor }}=$ $17.43 \mathrm{~min}, \mathrm{ee}=88 \%$. 


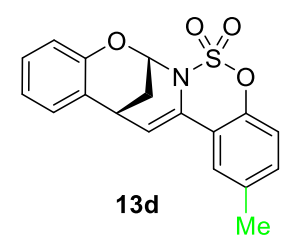

13d was synthesized following the general method (20 $\mathrm{mg}, 59 \%$ yield) as a white solid. ${ }^{1} \mathrm{H}$ NMR (500 MHz, $\left.\mathrm{CDCl}_{3}\right) \delta 7.33(\mathrm{~d}, J=0.9 \mathrm{~Hz}, 1 \mathrm{H}), 7.16-7.08(\mathrm{~m}, 3 \mathrm{H}), 7.02-$ $6.95(\mathrm{~m}, 2 \mathrm{H}), 6.90(\mathrm{td}, J=7.5,1.0 \mathrm{~Hz}, 1 \mathrm{H}), 6.45(\mathrm{dt}, J=2.7,2.2 \mathrm{~Hz}, 1 \mathrm{H}), 6.17(\mathrm{dd}, J=$ 7.6, $0.9 \mathrm{~Hz}, 1 \mathrm{H}), 3.71-3.61(\mathrm{~m}, 1 \mathrm{H}), 2.40-2.27(\mathrm{~m}, 4 \mathrm{H}), 2.13-2.10(\mathrm{~m}, 1 \mathrm{H}) .{ }^{13} \mathrm{C}$ NMR $\left(125 \mathrm{MHz}, \mathrm{CDCl}_{3}\right) \delta 151.1,146.2,135.9,131.2,131.1,128.2,127.9,125.0,124.2$ $121.3,119.0,118.3,117.4,108.5,76.9,27.4,25.6,21.0$. $[\alpha]_{D}^{20}-43.97$ (c $=1.25$ in $\mathrm{CHCl}_{3}$ ). ESI-HRMS: $[\mathrm{M}+\mathrm{H}]^{+}$calcd. For $\mathrm{C}_{18} \mathrm{H}_{16} \mathrm{NO}_{4} \mathrm{~S}^{+} \mathrm{m} / \mathrm{z}$ : 342.0795; found: 342.0794 . The enantiomeric excess was determined by HPLC analysis on Daicel Chiralpak IB column ( $n$-hexane/i-PrOH/dichloromethane $=80 / 17 / 3,1 \mathrm{~mL} / \mathrm{min}$ ), $\lambda=216 \mathrm{~nm}, t_{\text {major }}$ $=7.07 \mathrm{~min}, t_{\text {minor }}=10.17 \mathrm{~min}$, ee $=92 \%$.

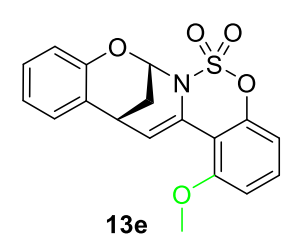

13e was synthesized following the general method ( $25 \mathrm{mg}, 70 \%$ yield) as a white solid. ${ }^{1} \mathrm{H}$ NMR $\left(500 \mathrm{MHz}, \mathrm{CDCl}_{3}\right) \delta 7.23(\mathrm{t}, J=8.3 \mathrm{~Hz}, 1 \mathrm{H}), 7.14-7.11(\mathrm{~m}, 2 \mathrm{H}), 7.02-$ $6.95(\mathrm{~m}, 2 \mathrm{H}), 6.90(\mathrm{td}, J=7.4,1.1 \mathrm{~Hz}, 1 \mathrm{H}), 6.82-6.77(\mathrm{~m}, 1 \mathrm{H}), 6.73(\mathrm{dd}, J=8.2,1.0 \mathrm{~Hz}$ $1 \mathrm{H}), 6.47-6.43(\mathrm{~m}, 1 \mathrm{H}), 3.88(\mathrm{~s}, 3 \mathrm{H}), 3.72-3.61(\mathrm{~m}, 1 \mathrm{H}), 2.30-2.25(\mathrm{~m}, 1 \mathrm{H}), 2.17-$ $2.06(\mathrm{~m}, 1 \mathrm{H}) .{ }^{13} \mathrm{C}$ NMR $\left(125 \mathrm{MHz}, \mathrm{CDCl}_{3}\right) \delta 157.9,151.4,149.6,129.7,128.5,128.3$, $128.2,125.4,121.4,117.4,116.0,111.9,109.4,109.1,77.5,56.3,28.2,25.6 .[\alpha]_{D}{ }^{20}$ -74.91 ( $c=0.75$ in $\mathrm{CHCl}_{3}$ ). ESI-HRMS: $[\mathrm{M}+\mathrm{H}]^{+}$calcd. For $\mathrm{C}_{18} \mathrm{H}_{16} \mathrm{NO}_{5} \mathrm{~S}^{+} \mathrm{m} / \mathrm{z}: 358.0744$; found: 358.0742. The enantiomeric excess was determined by HPLC analysis on Daicel Chiralpak IB column ( $n$-hexane $/ i-\mathrm{PrOH}=80 / 20,1 \mathrm{~mL} / \mathrm{min}$ ), $\lambda=210 \mathrm{~nm}, t_{\text {major }}=$ $9.03 \mathrm{~min}, t_{\text {minor }}=11.97 \mathrm{~min}$, ee $=96 \%$. 


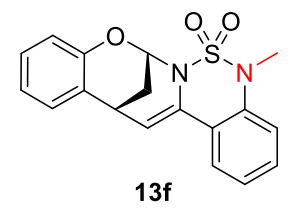

$13 \mathrm{f}$ was synthesized following the general method ( $24 \mathrm{mg}, 71 \%$ yield) as a white solid. ${ }^{1} \mathrm{H}$ NMR $\left(500 \mathrm{MHz}, \mathrm{CDCl}_{3}\right) \delta 7.53(\mathrm{~d}, J=8.0 \mathrm{~Hz}, 1 \mathrm{H}), 7.31(\mathrm{t}, J=7.6 \mathrm{~Hz}, 1 \mathrm{H}), 7.22(\mathrm{t}, J=$ $7.6 \mathrm{~Hz}, 1 \mathrm{H}), 7.17(\mathrm{~d}, J=7.9 \mathrm{~Hz}, 1 \mathrm{H}), 7.13-7.07(\mathrm{~m}, 2 \mathrm{H}), 6.98(\mathrm{~d}, J=7.9 \mathrm{~Hz}, 1 \mathrm{H}), 6.87$ (t, J = 7.4 Hz, 1H), $6.42(\mathrm{~s}, 1 \mathrm{H}), 5.94(\mathrm{~d}, J=7.1 \mathrm{~Hz}, 1 \mathrm{H}), 3.64(\mathrm{~d}, J=6.2 \mathrm{~Hz}, 1 \mathrm{H}), 3.14(\mathrm{~s}$, $3 \mathrm{H}), 2.32(\mathrm{~d}, J=12.8 \mathrm{~Hz}, 1 \mathrm{H}), 2.16(\mathrm{~d}, J=12.8 \mathrm{~Hz}, 1 \mathrm{H}) .{ }^{13} \mathrm{C} \mathrm{NMR}\left(125 \mathrm{MHz}, \mathrm{CDCl}_{3}\right) \delta$ 152.0, 140.3, 132.6, 130.1, 128.1, 127.8, 127.4, 126.2, 125.7, 125.0, 124.9, 121.1, $117.1,108.2,76.3,41.2,28.3,25.4 .[\alpha]_{D}{ }^{20}+80.94\left(c=0.33\right.$ in $\mathrm{CHCl}_{3}$ ). ESI-HRMS: $[\mathrm{M}+\mathrm{H}]^{+}$calcd. For $\mathrm{C}_{18} \mathrm{H}_{17} \mathrm{~N}_{2} \mathrm{O}_{3} \mathrm{~S}^{+} \mathrm{m} / \mathrm{z}$ : 341.0954; found: 341.0959. The enantiomeric excess was determined by HPLC analysis on Daicel Chiralpak IB column ( $n$-hexane $/ i-\mathrm{PrOH}=80 / 20,1 \mathrm{~mL} / \mathrm{min}), \lambda=210 \mathrm{~nm}, t_{\text {major }}=10.25 \mathrm{~min}, t_{\text {minor }}=15.40$ $\min$, ee $=96 \%$. 


\section{NMR spectra and HPLC traces}

In several HPLC figures, the retention time of the peaks in racemic samples is not consistent with that in chiral samples so well. It may due to the different solvent used in order to dissolve the samples well. 
${ }^{1} \mathrm{H}$ NMR of $6 \mathbf{a}, d_{\sigma}-$ DMSO

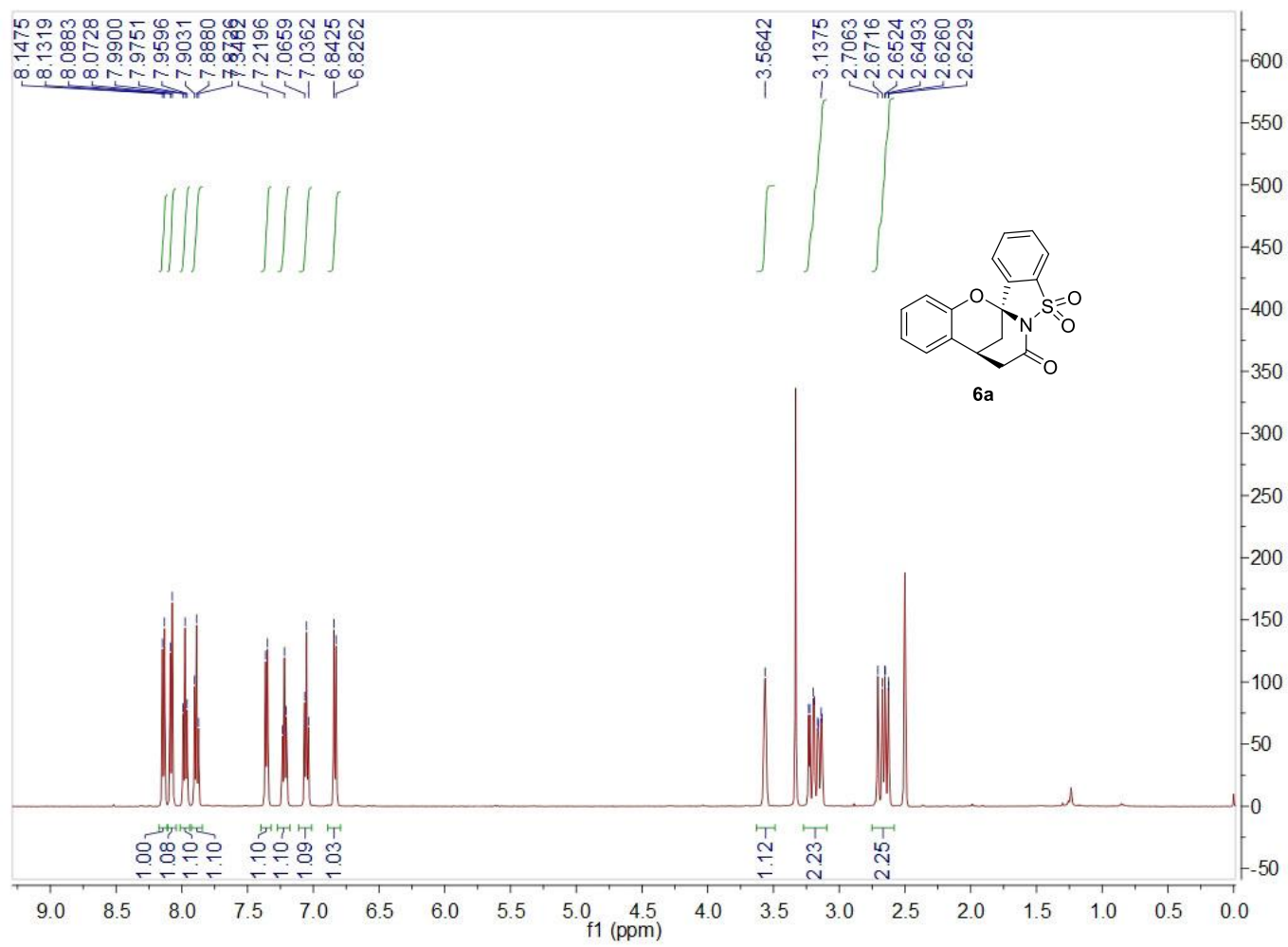

${ }^{13}$ C NMR of $6 a, d_{6}$-DMSO

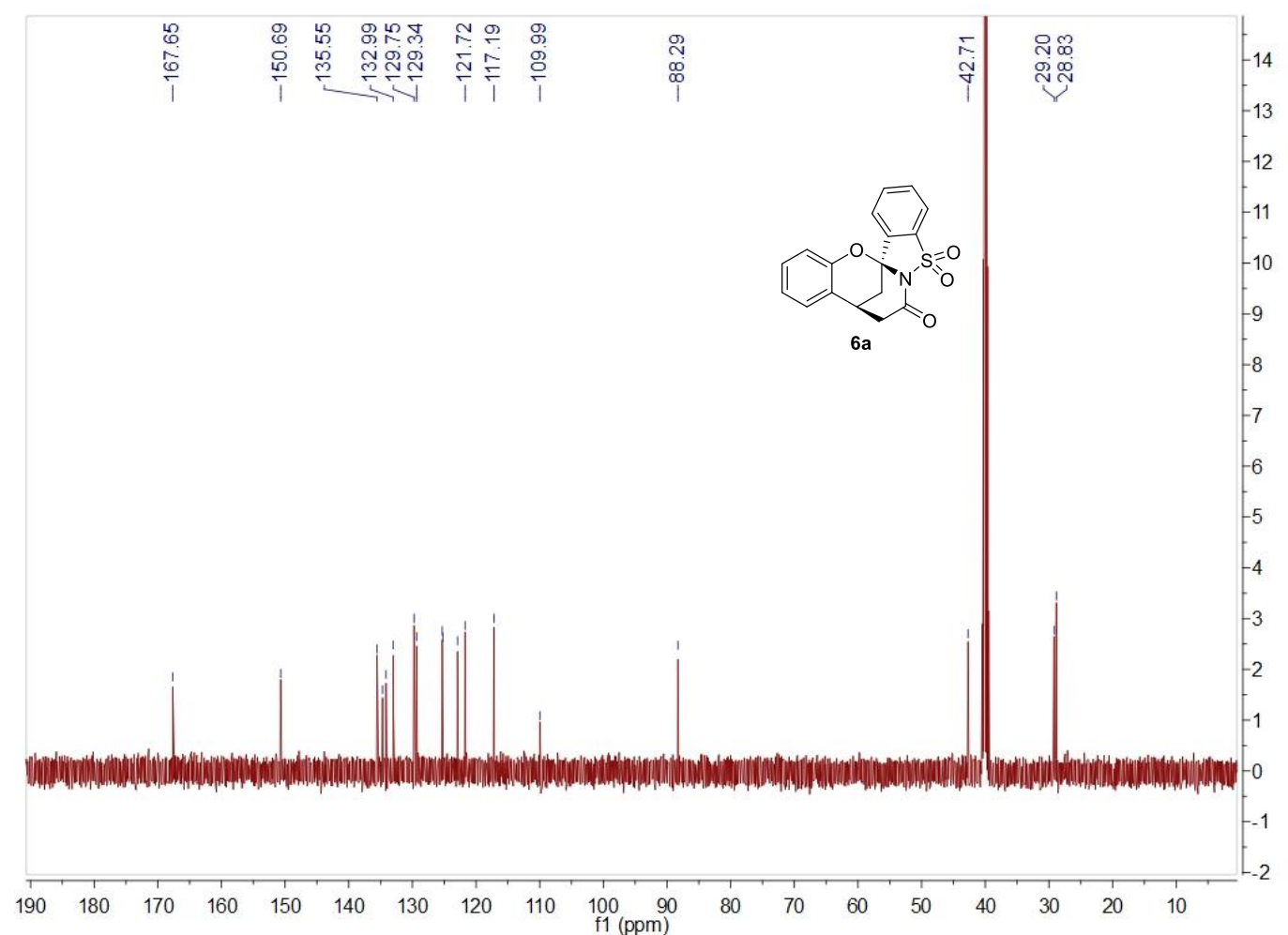




\section{Chiral HPLC analysis of rac-6a}

Chrom Type: Fixed WL Chromatogram, 227 nm

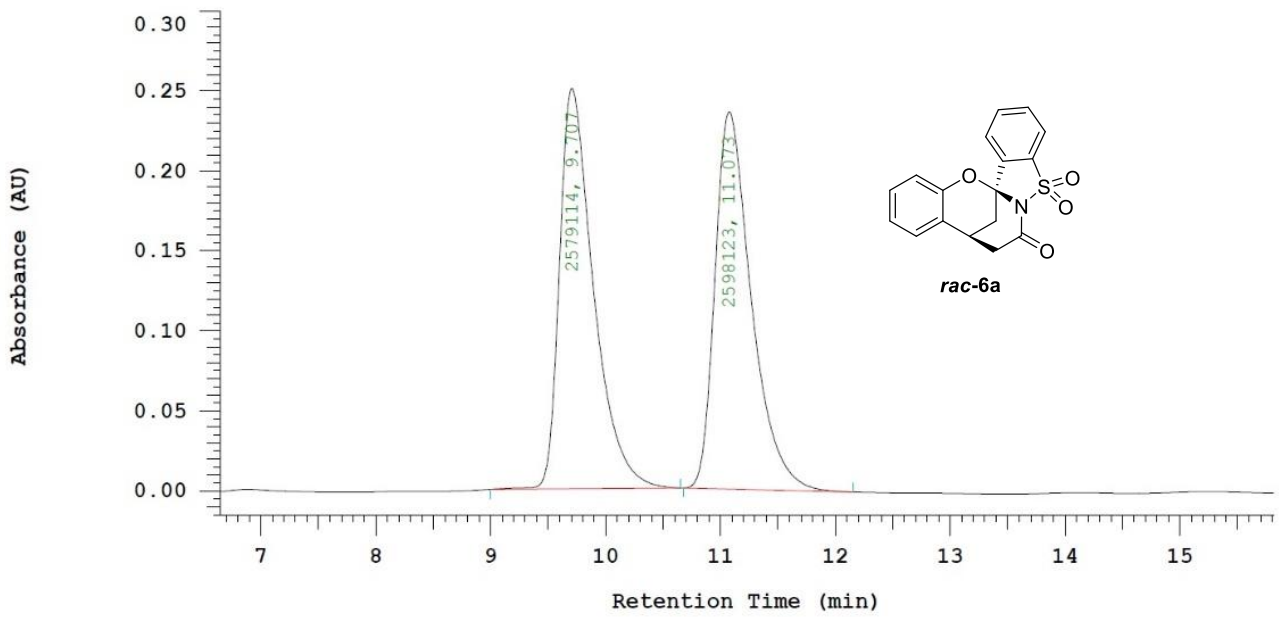

Chrom Type: Fixed WL Chromatogram, 227 nm

Peak Quantitation: AREA

Calculation Method: AREA응

\begin{tabular}{rrrrr}
\multicolumn{1}{c}{ RT } & Area & Area & BC \\
\hline 1 & 9.707 & 2579114 & 49.816 & BB \\
2 & 11.073 & 2598123 & 50.184 & BB \\
\hline & & 5177237 & 100.000 &
\end{tabular}

Chiral HPLC analysis of $6 a$

Chrom Type: Fixed WL Chromatogram, 225 nm

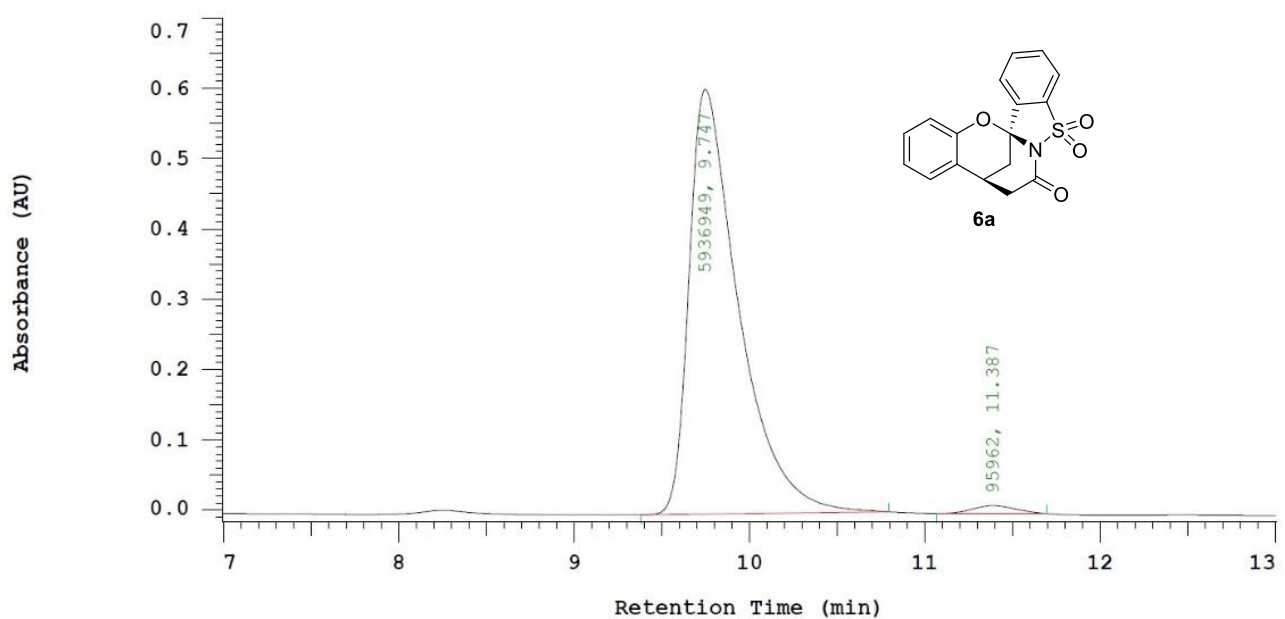

Chrom Type: Fixed WL Chromatogram, 225 nm

Peak Quantitation: AREA

Calculation Method: AREA음

\begin{tabular}{rrrrr}
\multicolumn{1}{c}{ RT } & \multicolumn{1}{c}{ Area } & Area & BC \\
\hline 1 & 9.747 & 5936949 & 98.409 & BB \\
2 & 11.387 & 95962 & 1.591 & BB \\
\hline & & 6032911 & 100.000 & \\
\hline
\end{tabular}


Chiral HPLC analysis of $6 a(1 \mathrm{mmol})$

Chrom Type: Fixed WL Chromatogram, $225 \mathrm{~nm}$

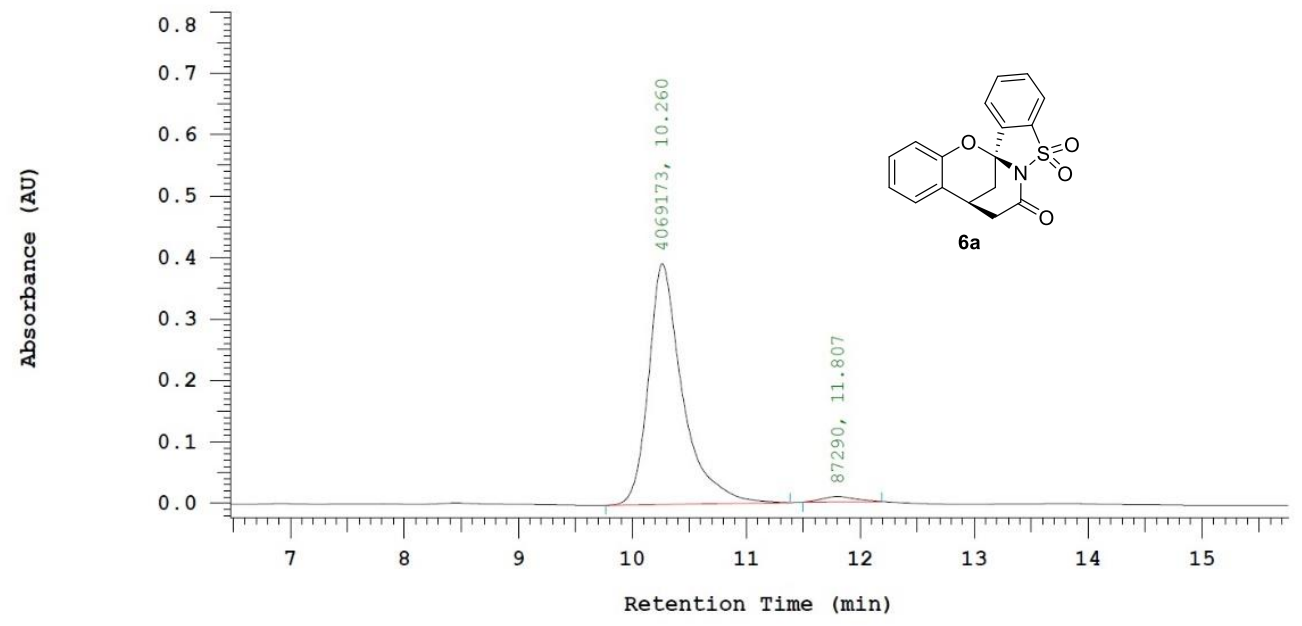

Chrom Type: Fixed WL Chromatogram, 225 nm

Peak Quantitation: AREA

Calculation Method: AREA응

\begin{tabular}{|c|c|c|c|c|}
\hline No. & $\mathrm{RT}$ & Area & Area 응 & $\mathrm{BC}$ \\
\hline 1 & 10.260 & 4069173 & 97.900 & BB \\
\hline 2 & 11.807 & 87290 & 2.100 & BB \\
\hline & & 4156463 & 100.000 & \\
\hline
\end{tabular}

${ }^{1} \mathrm{H}$ NMR of $6 \mathbf{b}, d_{6}$-DMSO

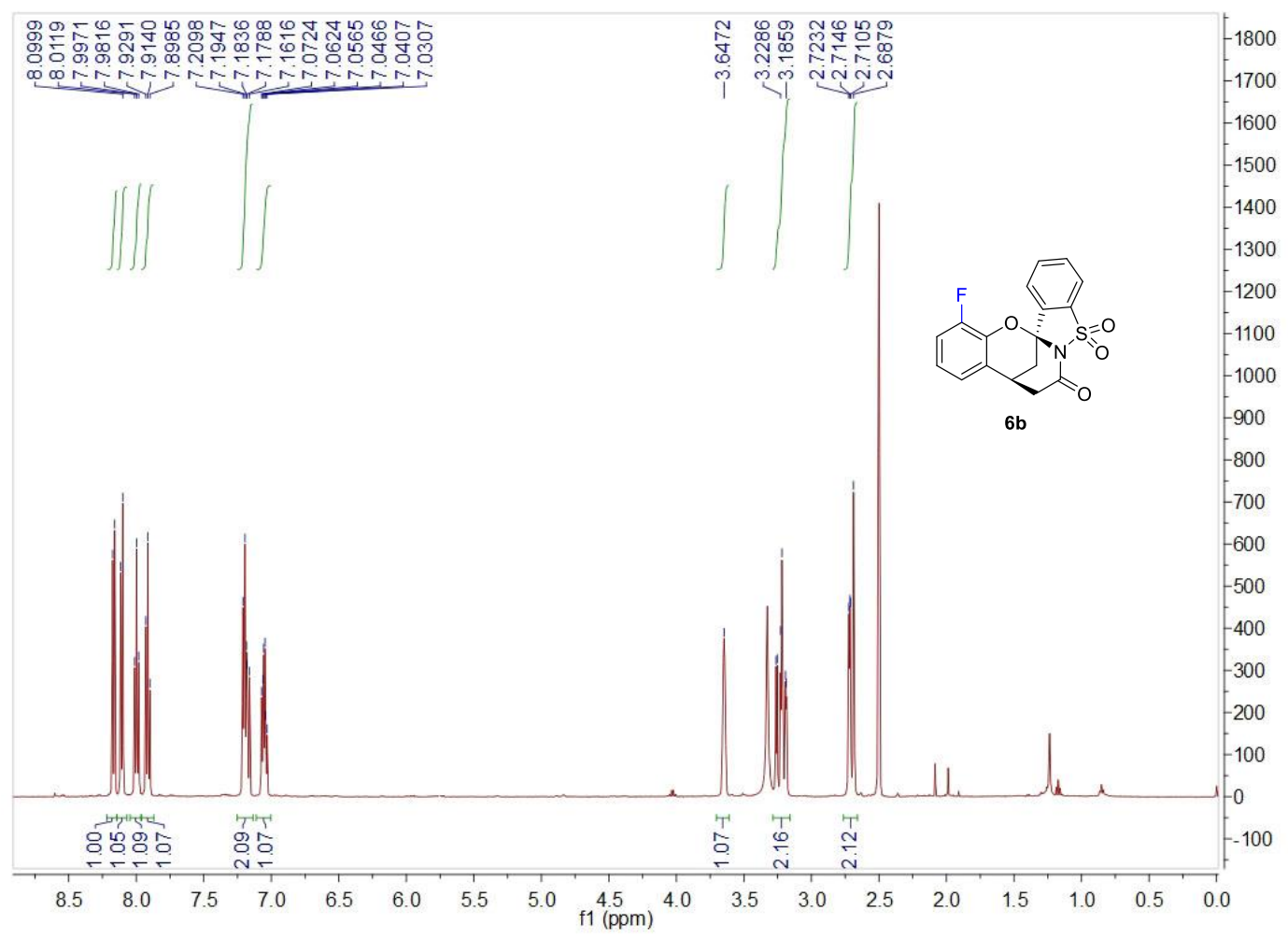




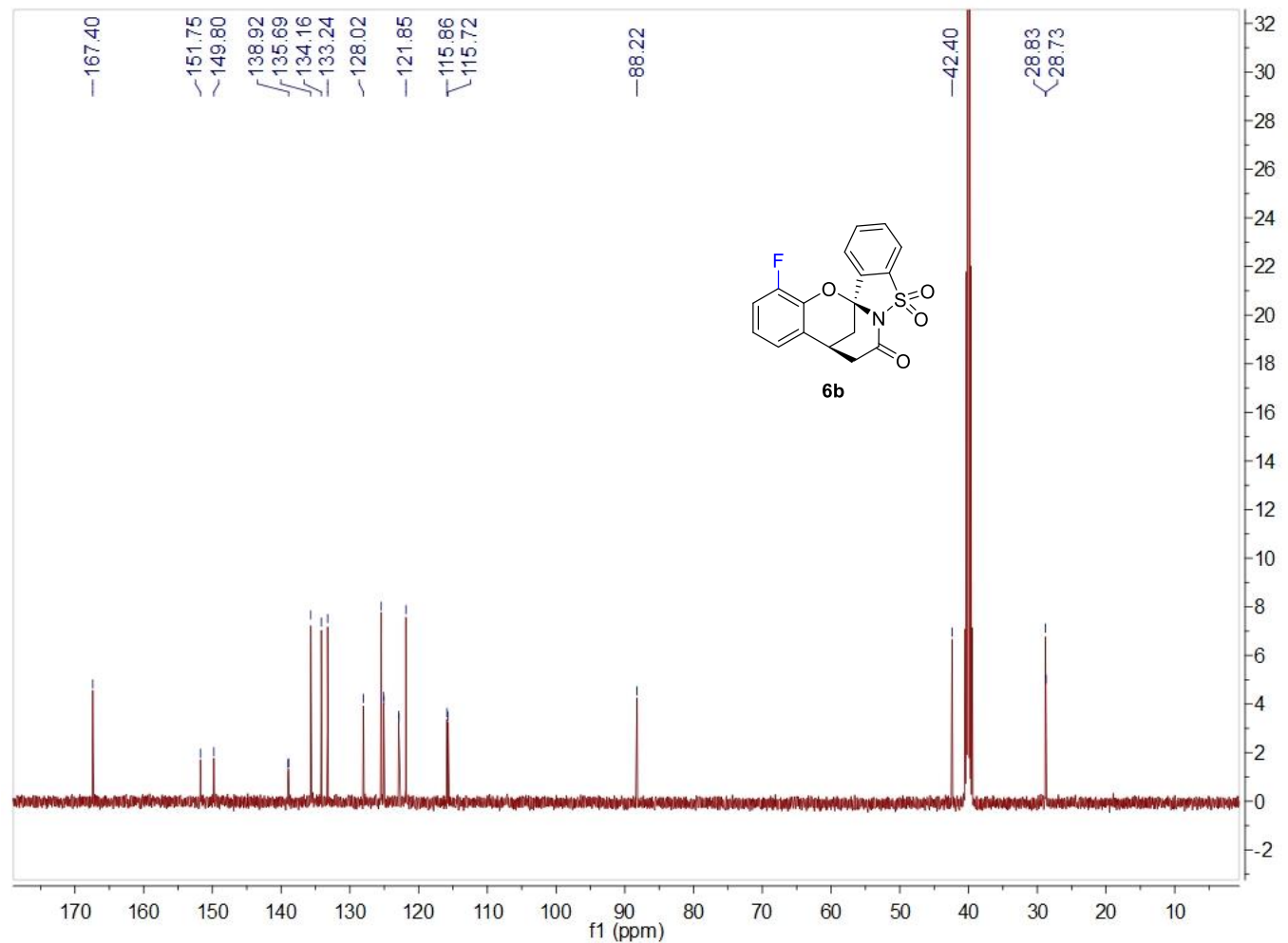

Chiral HPLC analysis of rac-6b

Chrom Type: Fixed WL Chromatogram, 225 nm

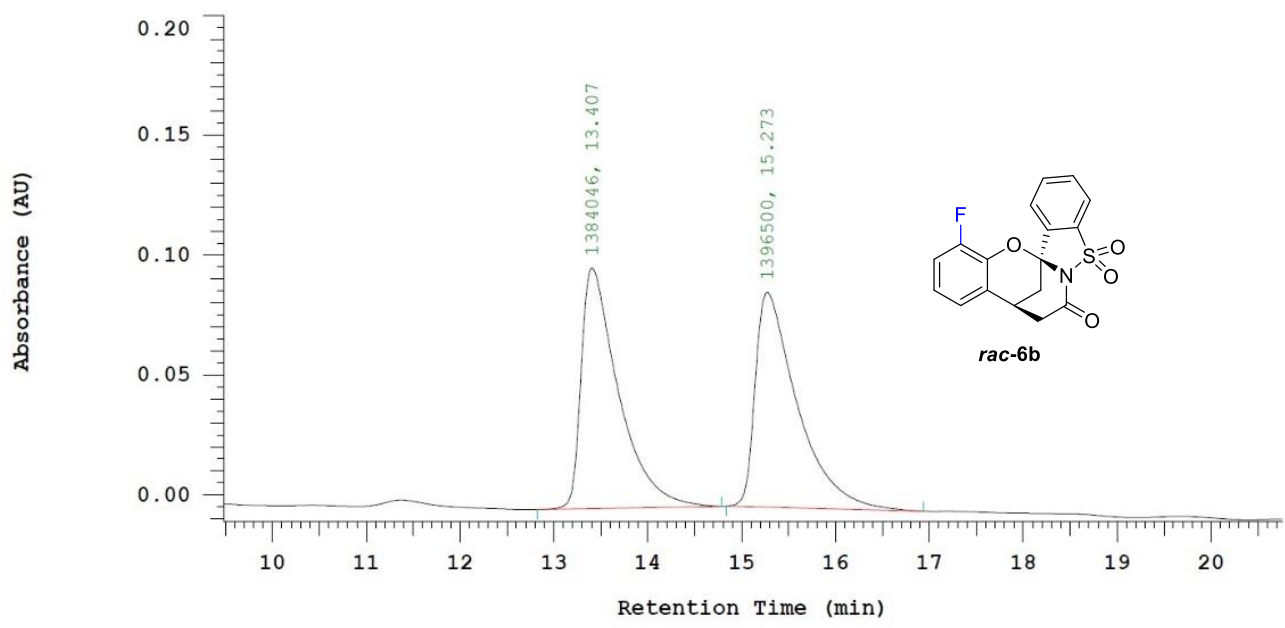

Chrom Type: Fixed WL Chromatogram, $225 \mathrm{~nm}$ Peak Quantitation: AREA

Calculation Method: AREA응

\begin{tabular}{ccccc} 
No. & RT & Area & Area 은 & BC \\
\hline 1 & 13.407 & 1384046 & 49.776 & BB \\
2 & 15.273 & 1396500 & 50.224 & BB \\
\hline & & 2780546 & 100.000 & \\
\hline
\end{tabular}

Chiral HPLC analysis of $\mathbf{6 b}$ 
Chrom Type: Fixed WL Chromatogram, 225 nm

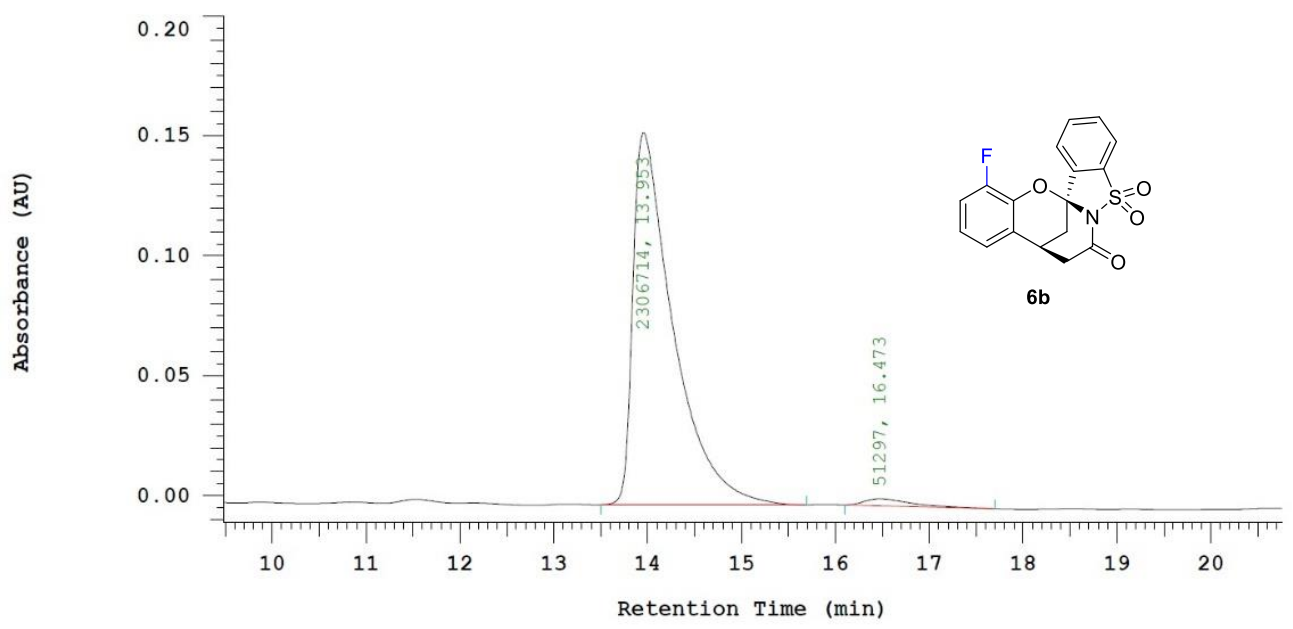

Chrom Type: Fixed WL Chromatogram, $225 \mathrm{~nm}$

Peak Quantitation: AREA

Calculation Method: AREA\%

\begin{tabular}{|c|c|c|c|c|}
\hline No. & $\mathrm{RT}$ & Area & Area \% & $\mathrm{BC}$ \\
\hline 1 & 13.953 & 2306714 & 97.825 & $\mathrm{BB}$ \\
\hline \multirow[t]{2}{*}{2} & 16.473 & 51297 & 2.175 & BB \\
\hline & & 2358011 & 100.000 & \\
\hline
\end{tabular}

${ }^{1} \mathrm{H}$ NMR of $6 \mathbf{c}, d_{6}$-DMSO

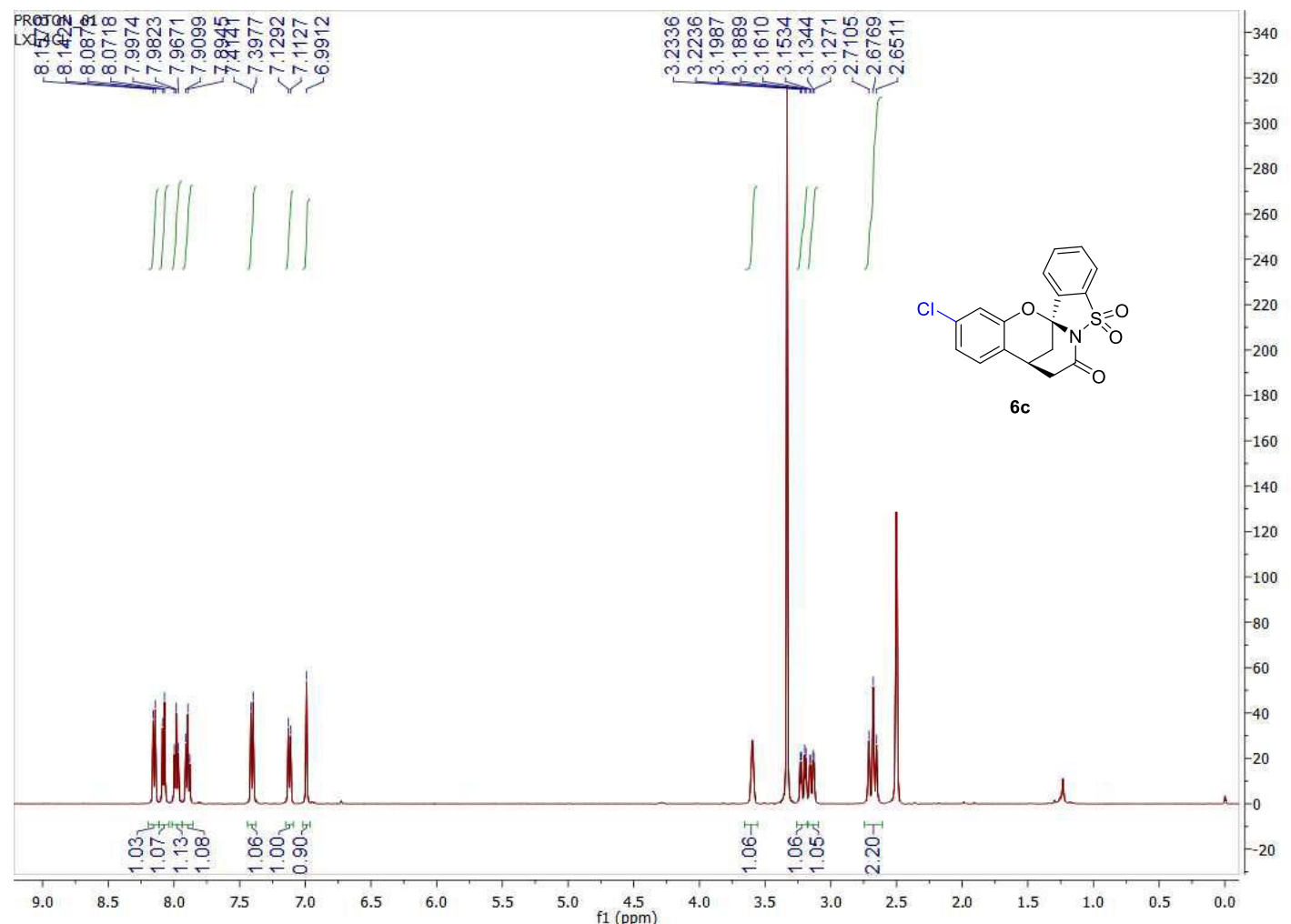

${ }^{13} \mathrm{C}$ NMR of $6 \mathrm{c}, d_{6}$-DMSO 


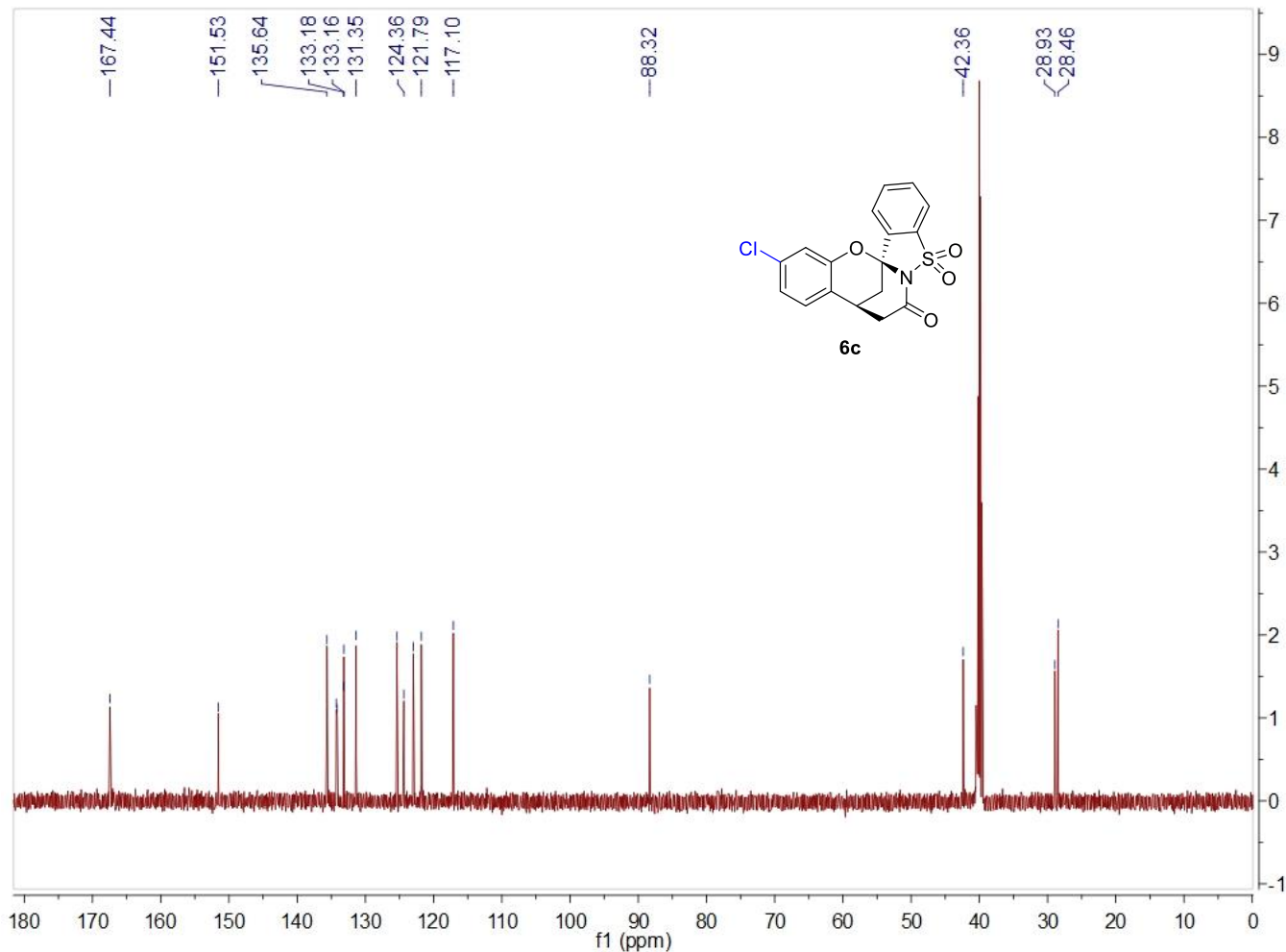

Chiral HPLC analysis of rac-6c

Chrom Type: Fixed WL Chromatogram, 225 nm

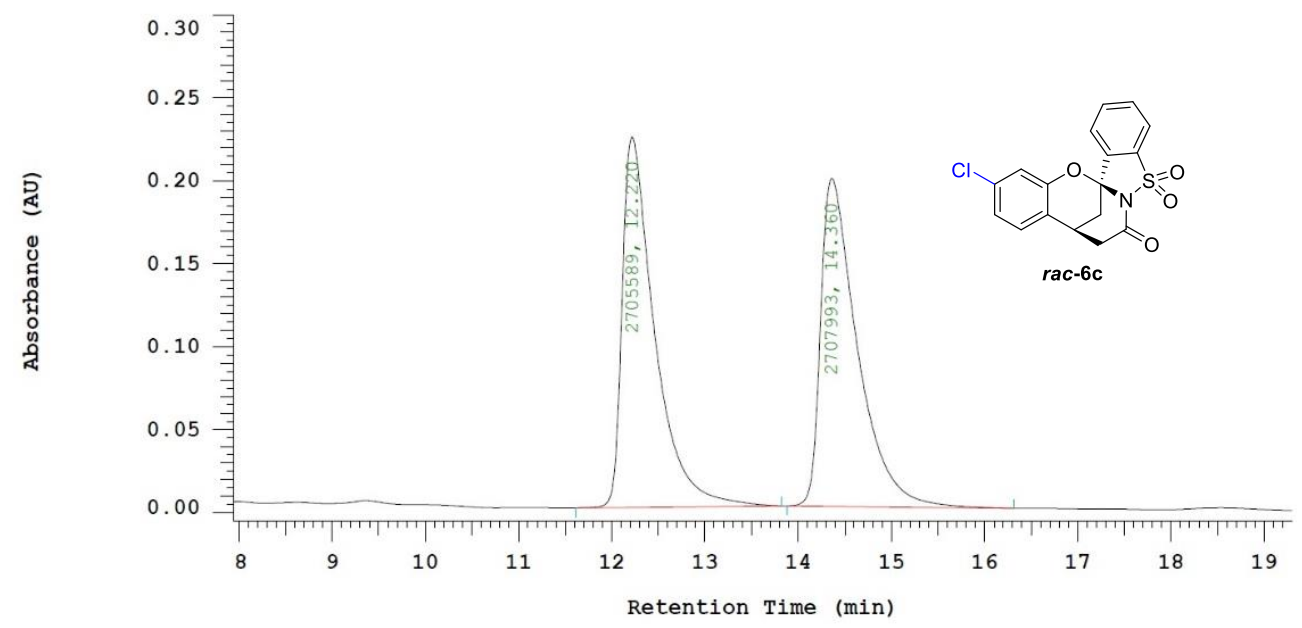

Chrom Type: Fixed WL Chromatogram, $225 \mathrm{~nm}$

Peak Quantitation: AREA

Calculation Method: AREA\%

\begin{tabular}{ccccc} 
No. & RT & Area & Area o & BC \\
\hline 1 & 12.220 & 2705589 & 49.978 & BB \\
2 & 14.360 & 2707993 & 50.022 & BB \\
\hline & 5413582 & 100.000 &
\end{tabular}

Chiral HPLC analysis of 6c 
Chrom Type: Fixed WL Chromatogram, $225 \mathrm{~nm}$

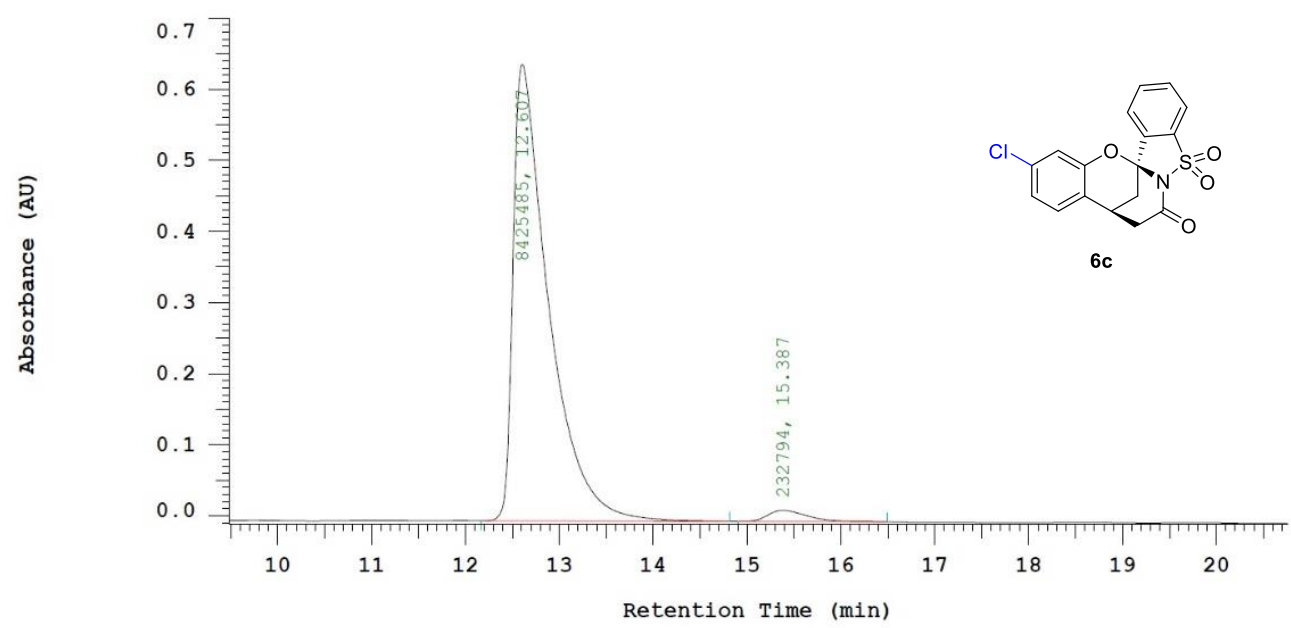

Chrom Type: Fixed WL Chromatogram, $225 \mathrm{~nm}$

Peak Quantitation: AREA

Calculation Method: AREA응

\begin{tabular}{|c|c|c|c|c|}
\hline No. & $\mathrm{RT}$ & Area & Area 응 & $\mathrm{BC}$ \\
\hline 1 & 12.607 & 8425485 & 97.311 & BB \\
\hline 2 & 15.387 & 232794 & 2.689 & BB \\
\hline & & 8658279 & 100.000 & \\
\hline
\end{tabular}

${ }^{1} \mathrm{H}$ NMR of $6 \mathbf{d}, d_{6}$-DMSO

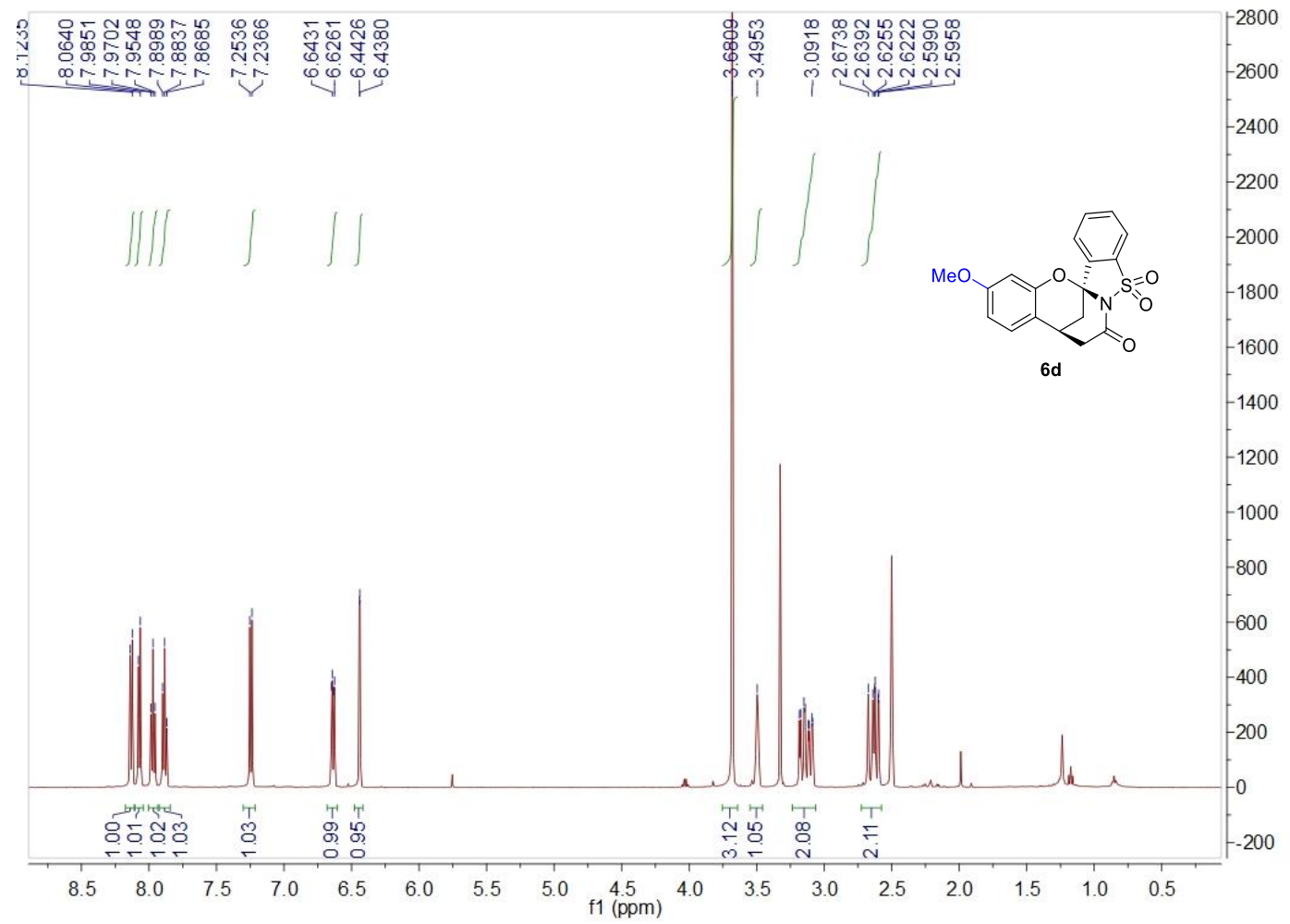

${ }^{13} \mathrm{C}$ NMR of $6 \mathbf{d}, d_{6}$-DMSO 


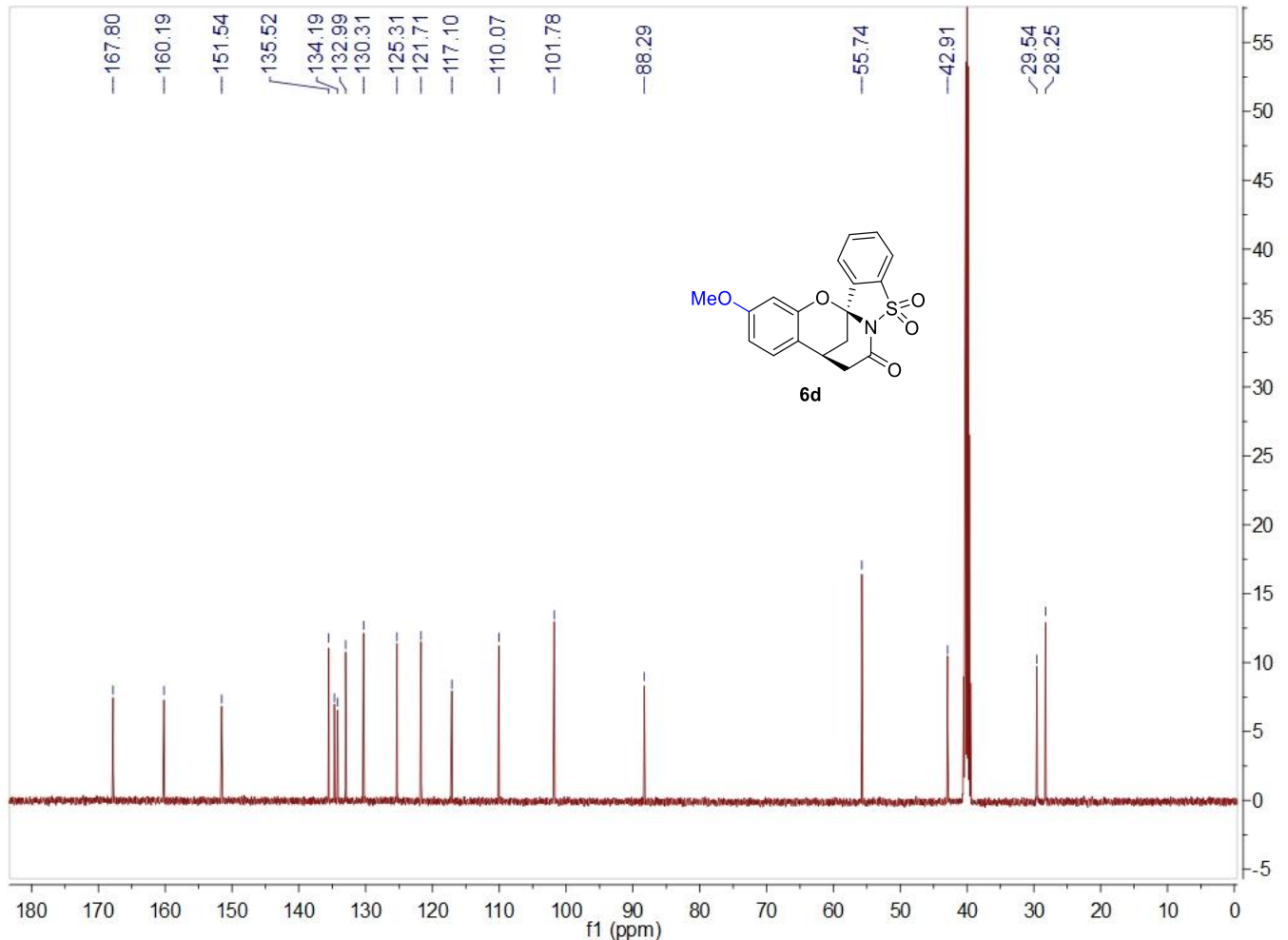

Chiral HPLC analysis of rac-6d

Chrom Type: Fixed WL Chromatogram, $225 \mathrm{~nm}$

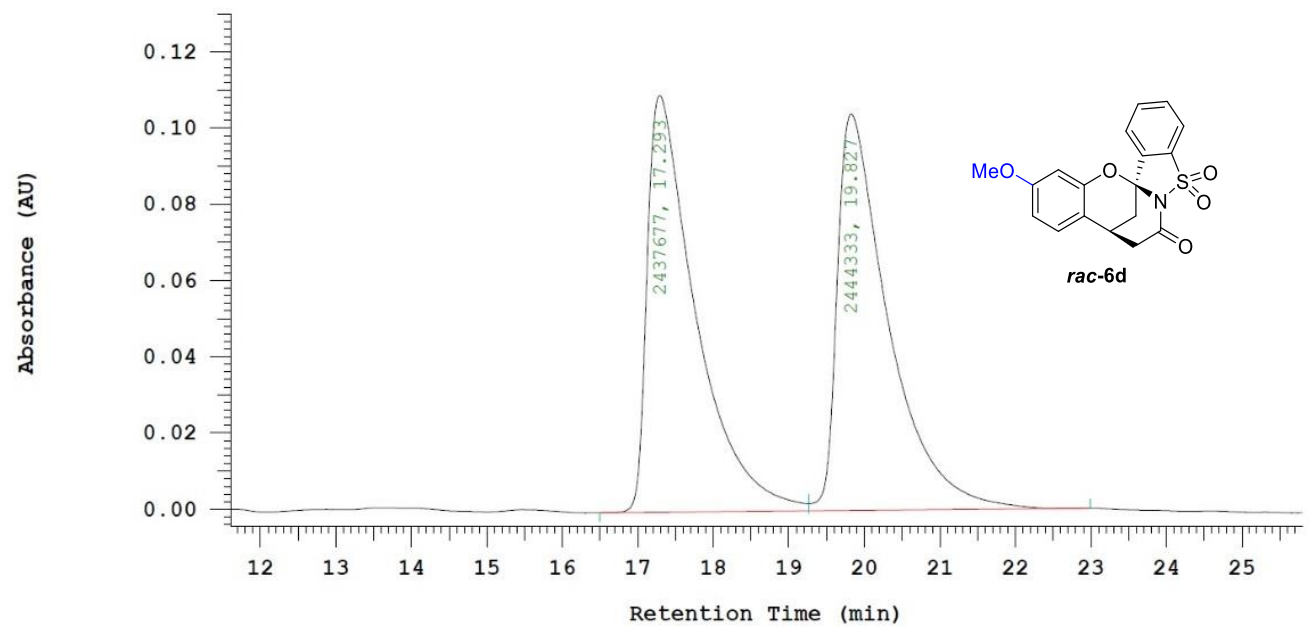

Chrom Type: Fixed WL Chromatogram, $225 \mathrm{~nm}$

Peak Quantitation: AREA

Calculation Method: AREA응

\begin{tabular}{ccccc} 
No. & RT & Area & Area $\%$ & BC \\
\hline 1 & 17.293 & 2437677 & 49.932 & BV \\
2 & 19.827 & 244433 & 50.068 & VB \\
\hline & & 4882010 & 100.000
\end{tabular}

Chiral HPLC analysis of $\mathbf{6 d}$ 
Chrom Type: Fixed WL Chromatogram, 225 nm

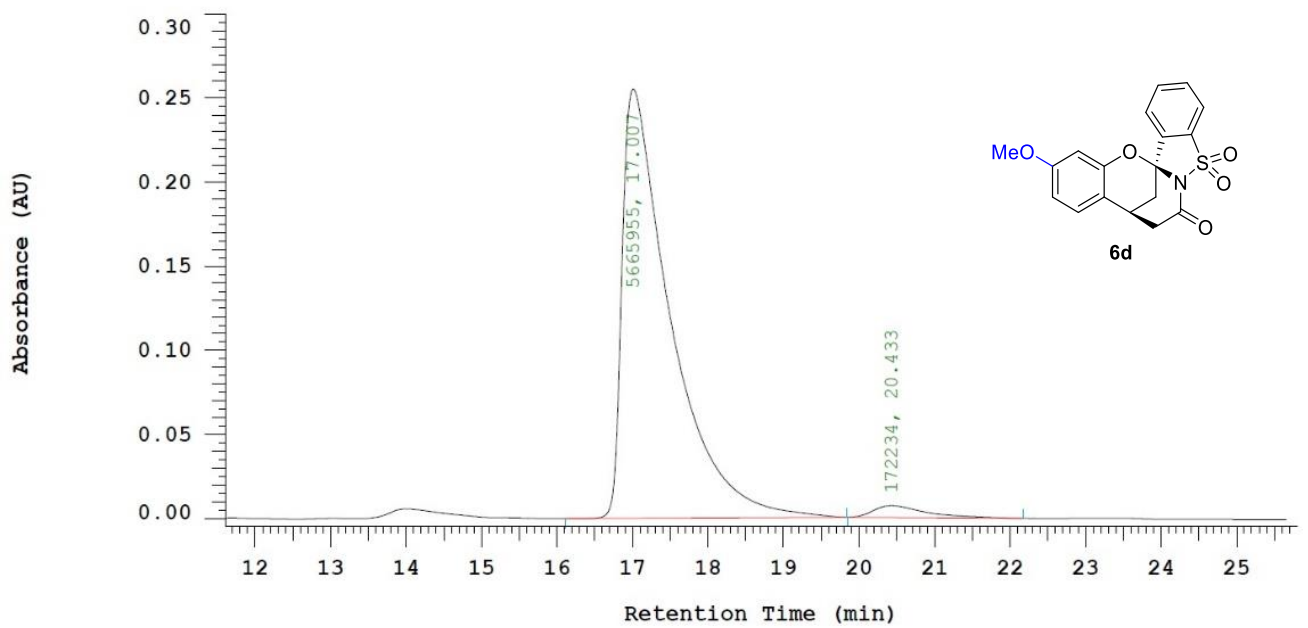

Chrom Type: Fixed WL Chromatogram, $225 \mathrm{~nm}$

Peak Quantitation: AREA

Calculation Method: AREA음

\begin{tabular}{|c|c|c|c|c|}
\hline No. & RT & Area & Area 응 & $\mathrm{BC}$ \\
\hline 1 & 17.007 & 5665955 & 97.050 & $\mathrm{BB}$ \\
\hline 2 & 20.433 & 172234 & 2.950 & $\mathrm{BB}$ \\
\hline & & 5838189 & 100.000 & \\
\hline
\end{tabular}

${ }^{1} \mathrm{H}$ NMR of $6 \mathbf{e}, d_{6}$-DMSO

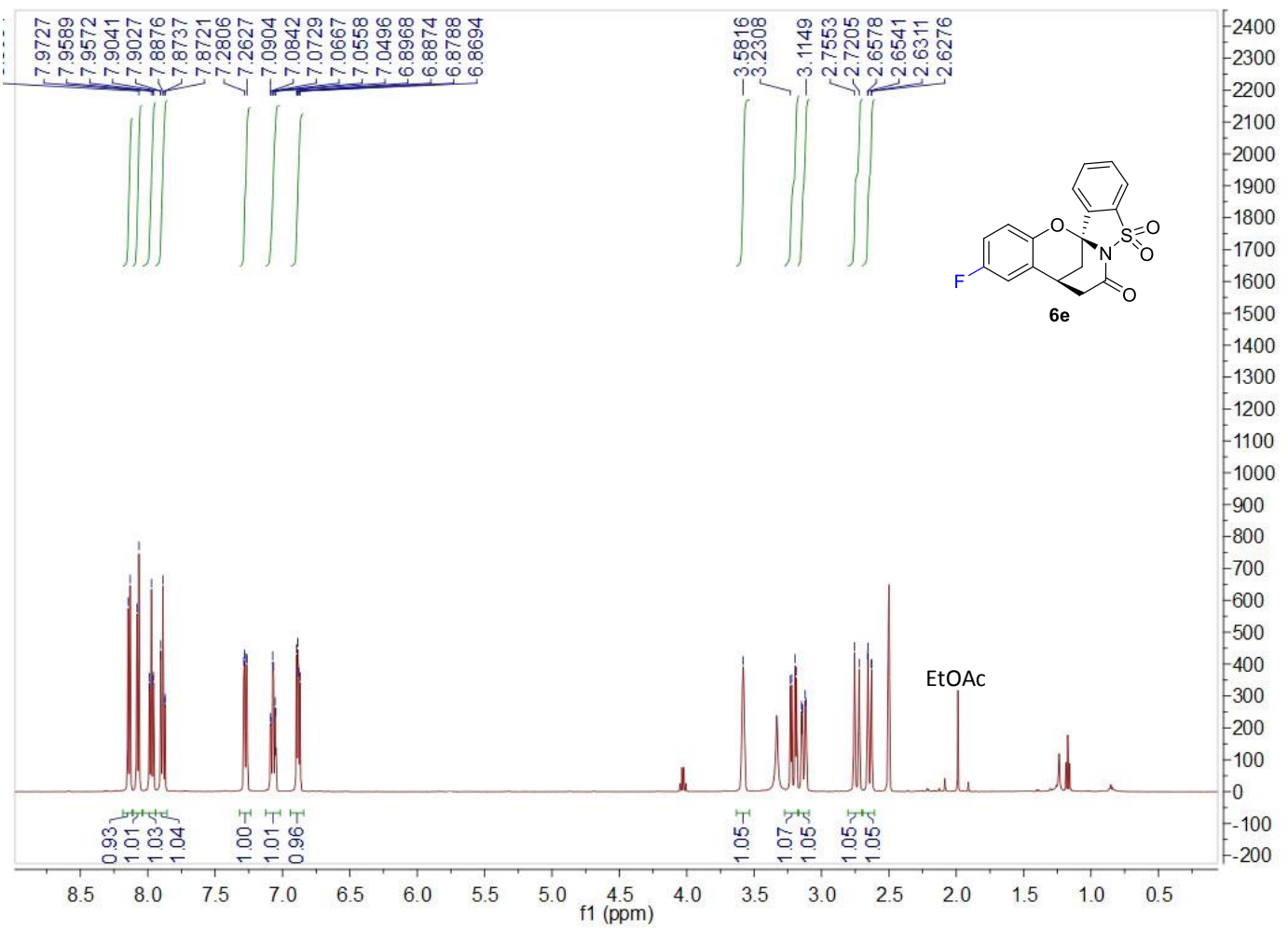


${ }^{13}$ C NMR of $6 e, d_{6}$-DMSO

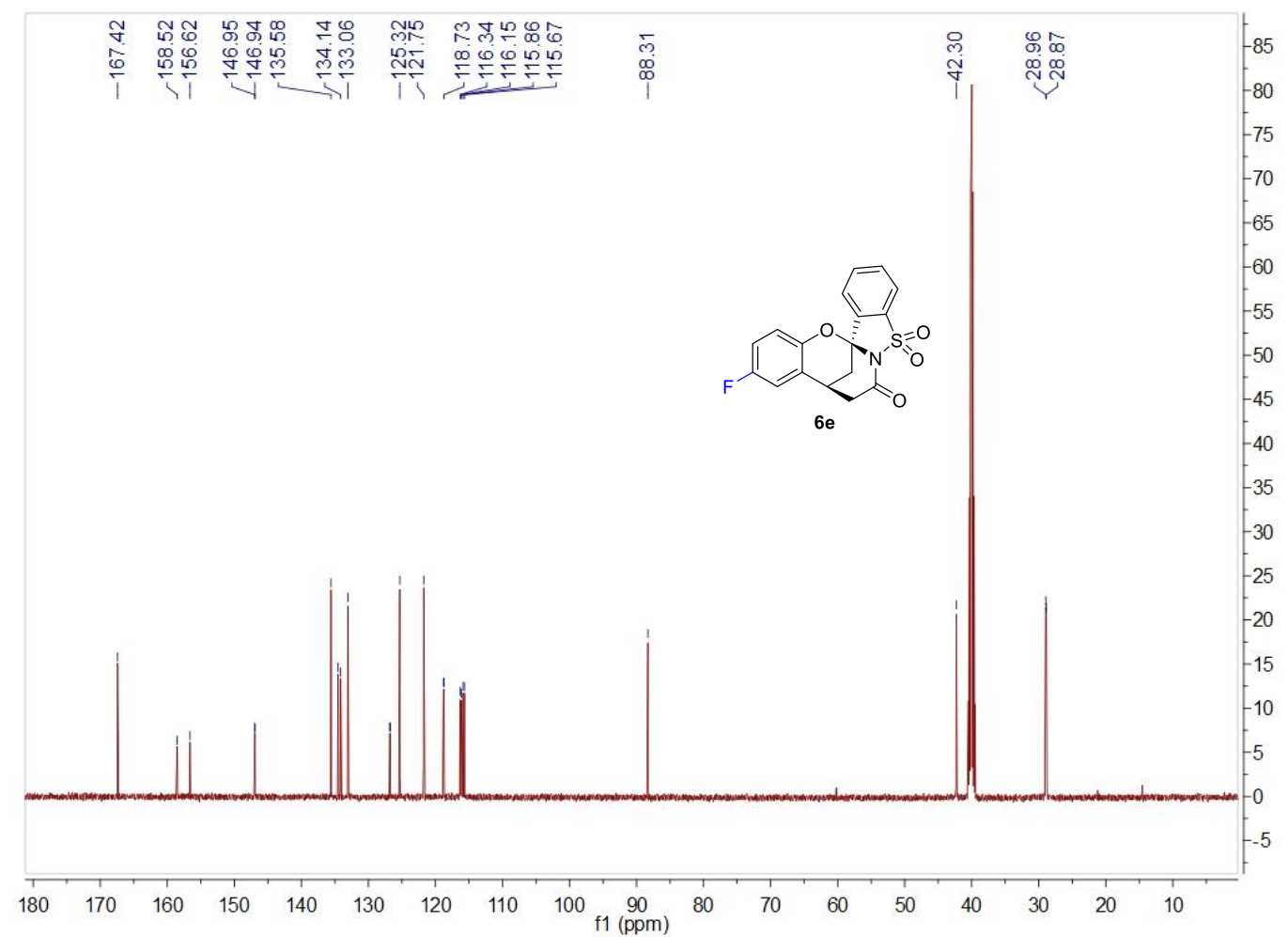

Chiral HPLC analysis of rac-6e

Chrom Type: Fixed WL Chromatogram, 225 nm

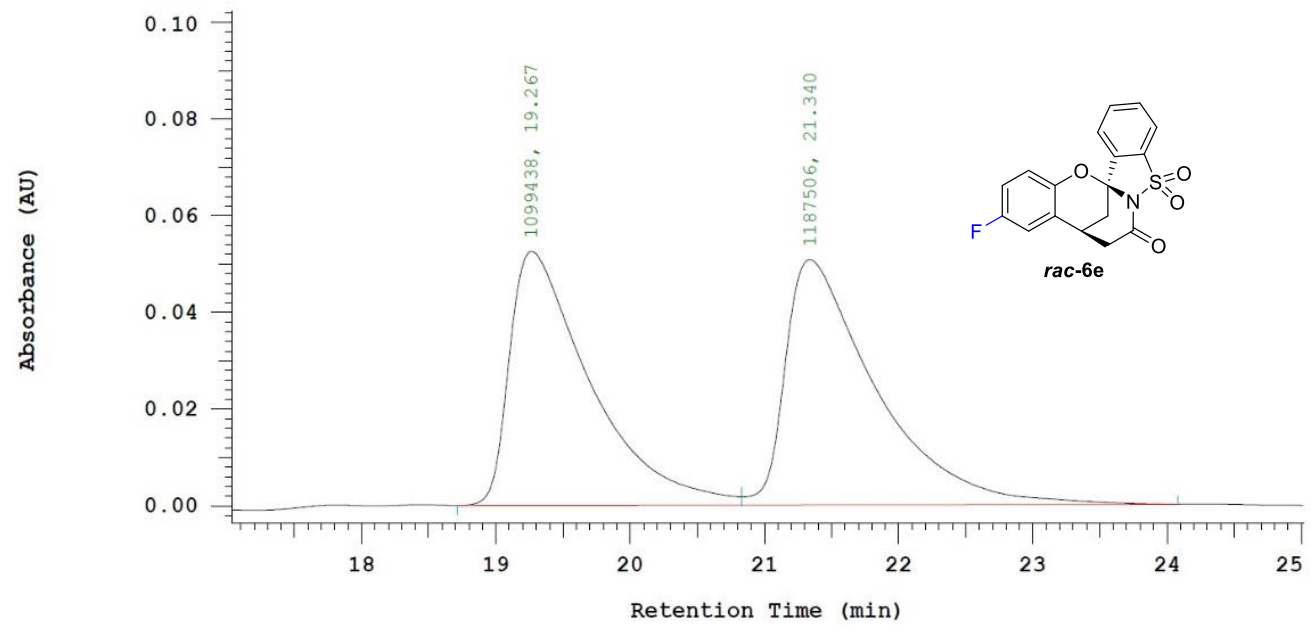

Chrom Type: Fixed WL Chromatogram, $225 \mathrm{~nm}$

Peak Quantitation: AREA

Calculation Method: AREA응

\begin{tabular}{|c|c|c|c|c|}
\hline No. & RT & Area & Area \% & $\mathrm{BC}$ \\
\hline 1 & 19.267 & 1099438 & 48.075 & BV \\
\hline 2 & 21.340 & 1187506 & 51.925 & VB \\
\hline & & 2286944 & 100.000 & \\
\hline
\end{tabular}




\section{Chiral HPLC analysis of $6 e$}

Chrom Type: Fixed WL Chromatogram, 225 nm

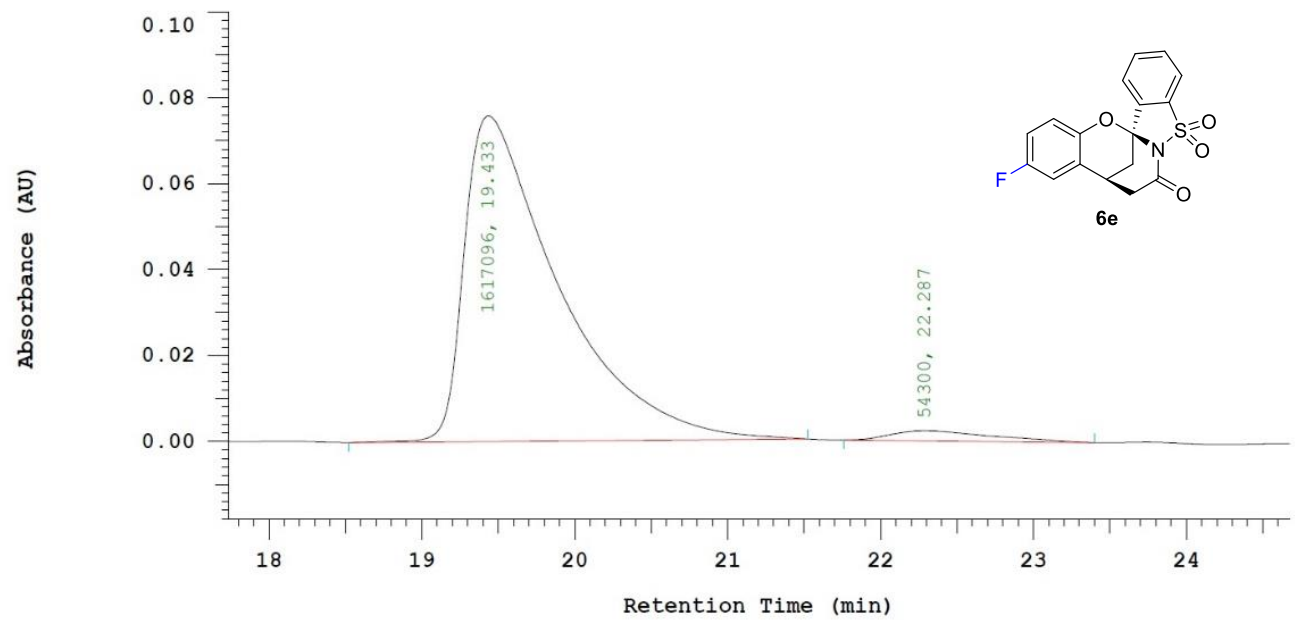

Chrom Type: Fixed WL Chromatogram, $225 \mathrm{~nm}$

Peak Quantitation: AREA

Calculation Method: AREA응

\begin{tabular}{rrrrr} 
No. & \multicolumn{1}{c}{ RT } & Area & Area $\%$ & BC \\
\hline 1 & 19.433 & 1617096 & 96.751 & BB \\
2 & 22.287 & 54300 & 3.249 & BB \\
\hline & & 1671396 & 100.000
\end{tabular}

\section{${ }^{1} \mathrm{H}$ NMR of $\mathbf{6 f}, \mathrm{CDCl}_{3}$}

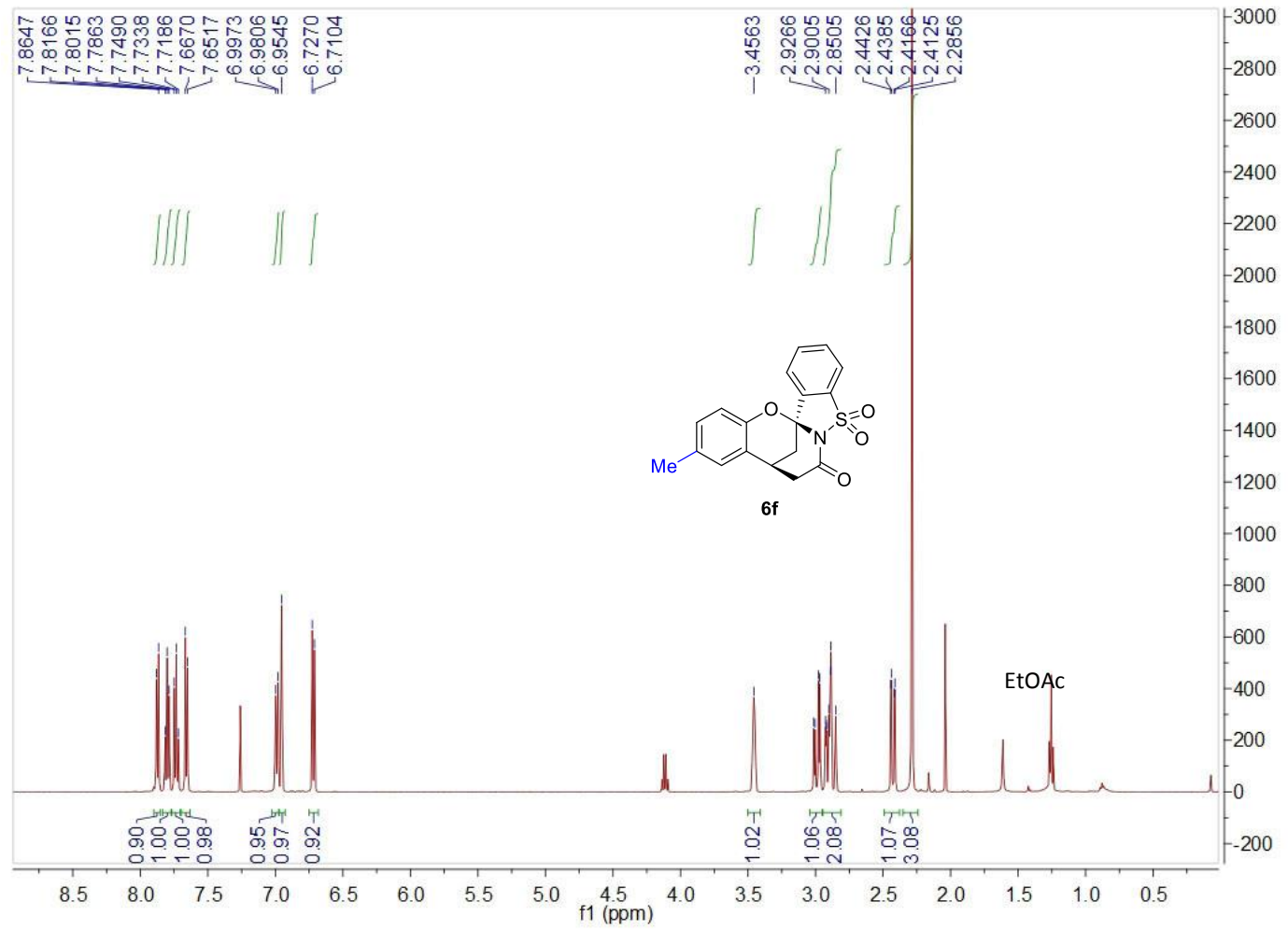


${ }^{13} \mathrm{CNMR}$ of $6 \mathrm{f}, \mathrm{CDCl}_{3}$

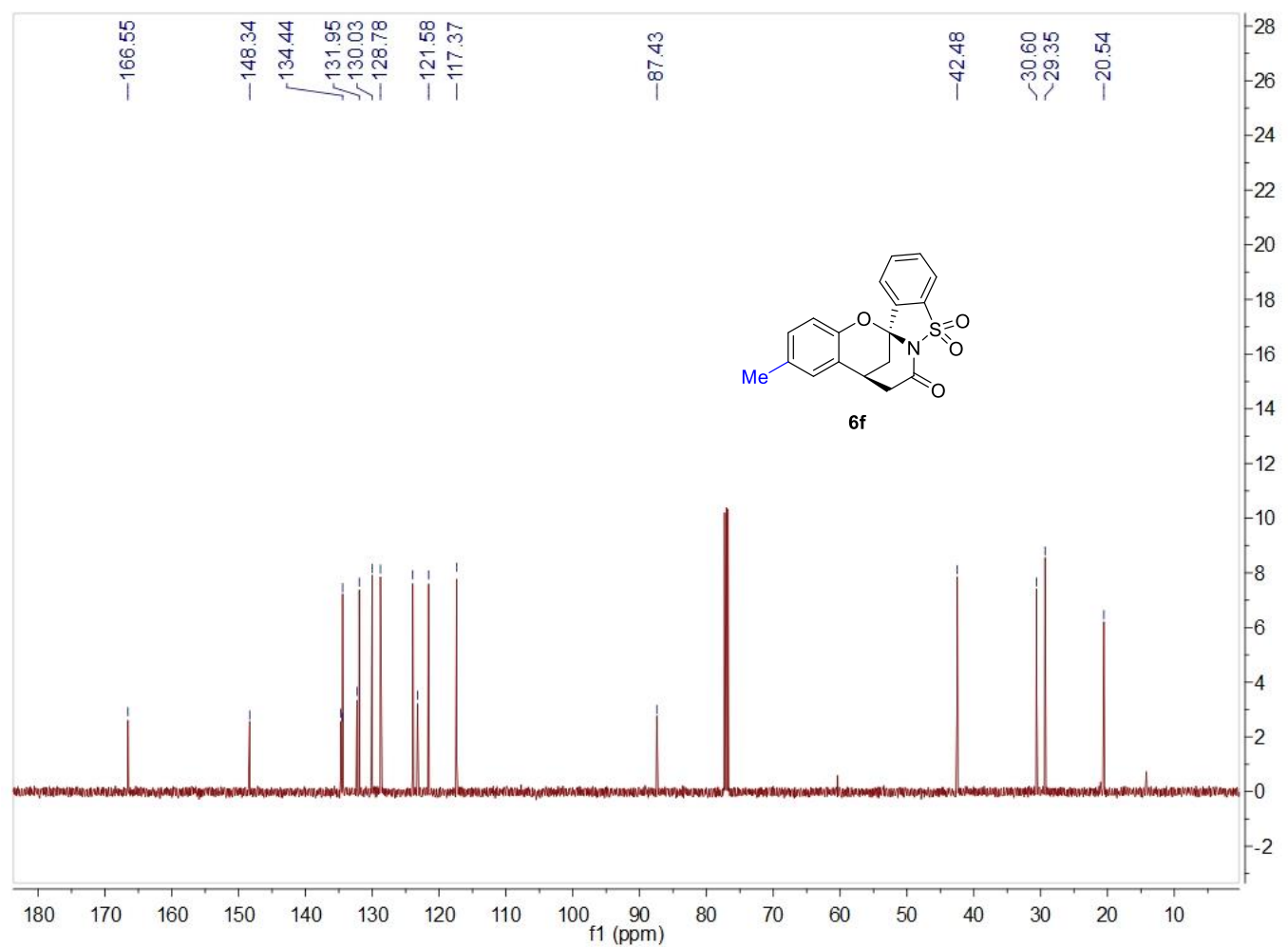

Chiral HPLC analysis of rac-6f

Chrom Type: Fixed WL Chromatogram, $225 \mathrm{~nm}$

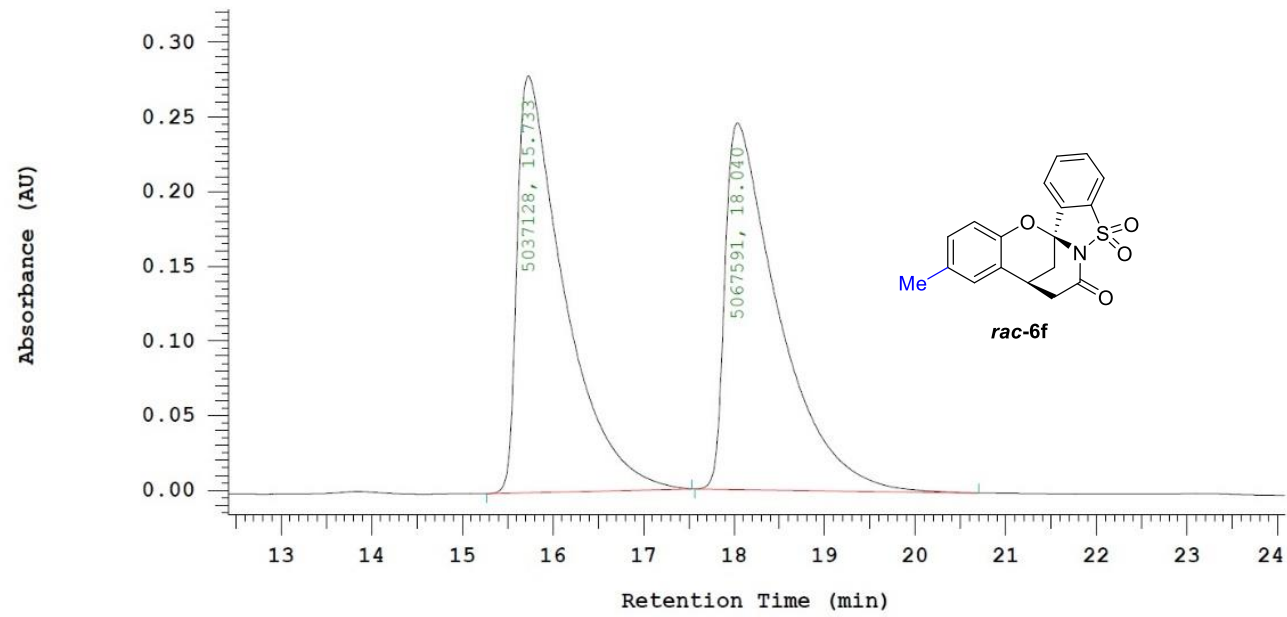

Chrom Type: Fixed WL Chromatogram, $225 \mathrm{~nm}$

Peak Quantitation: AREA

Calculation Method: AREA응

\begin{tabular}{ccccc} 
No. & RT & Area & Area & BC \\
\hline 1 & 15.733 & 5037128 & 49.849 & BB \\
2 & 18.040 & 5067591 & 50.151 & BB \\
\hline & & 10104719 & 100.000
\end{tabular}




\section{Chiral HPLC analysis of $6 f$}

Chrom Type: Fixed WL Chromatogram, $225 \mathrm{~nm}$

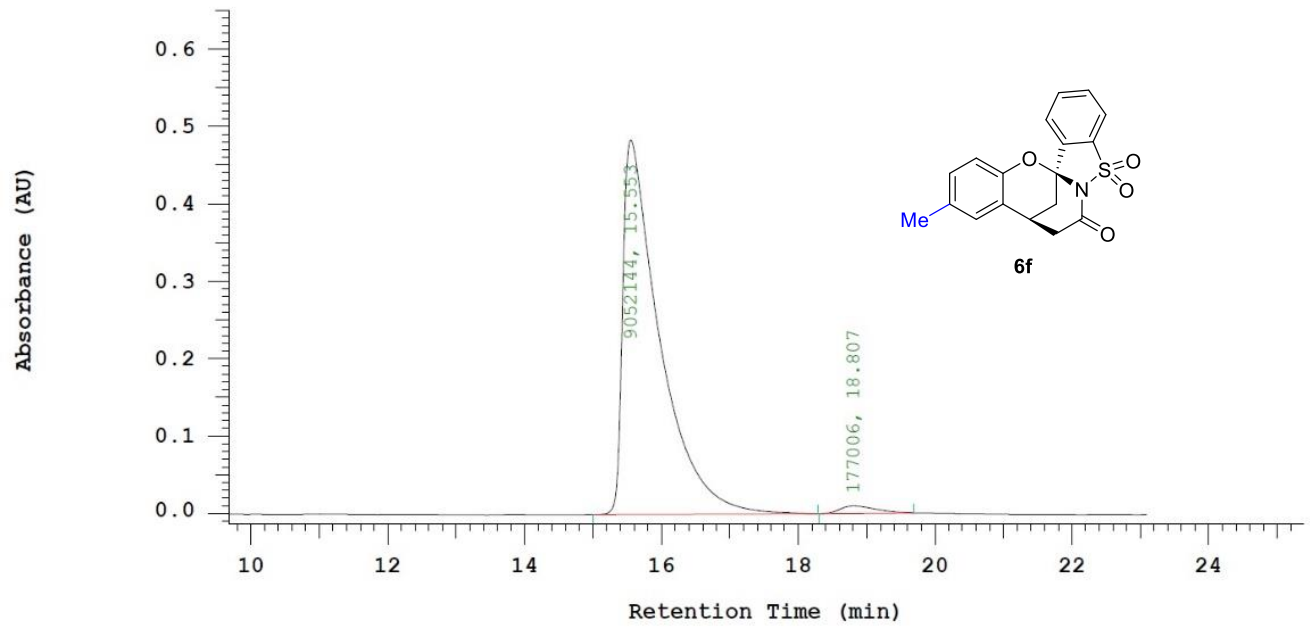

Chrom Type: Fixed WL Chromatogram, $225 \mathrm{~nm}$

Peak Quantitation: AREA

Calculation Method: AREA응

\begin{tabular}{|c|c|c|c|c|}
\hline No. & $\mathrm{RT}$ & Area & Area 응 & $\mathrm{BC}$ \\
\hline 1 & 15.553 & 9052144 & 98.082 & BB \\
\hline 2 & 18.807 & 177006 & 1.918 & BB \\
\hline & & 9229150 & 100.000 & \\
\hline
\end{tabular}

${ }^{1} \mathrm{H}$ NMR of $6 \mathrm{~g}, d_{6}$-DMSO

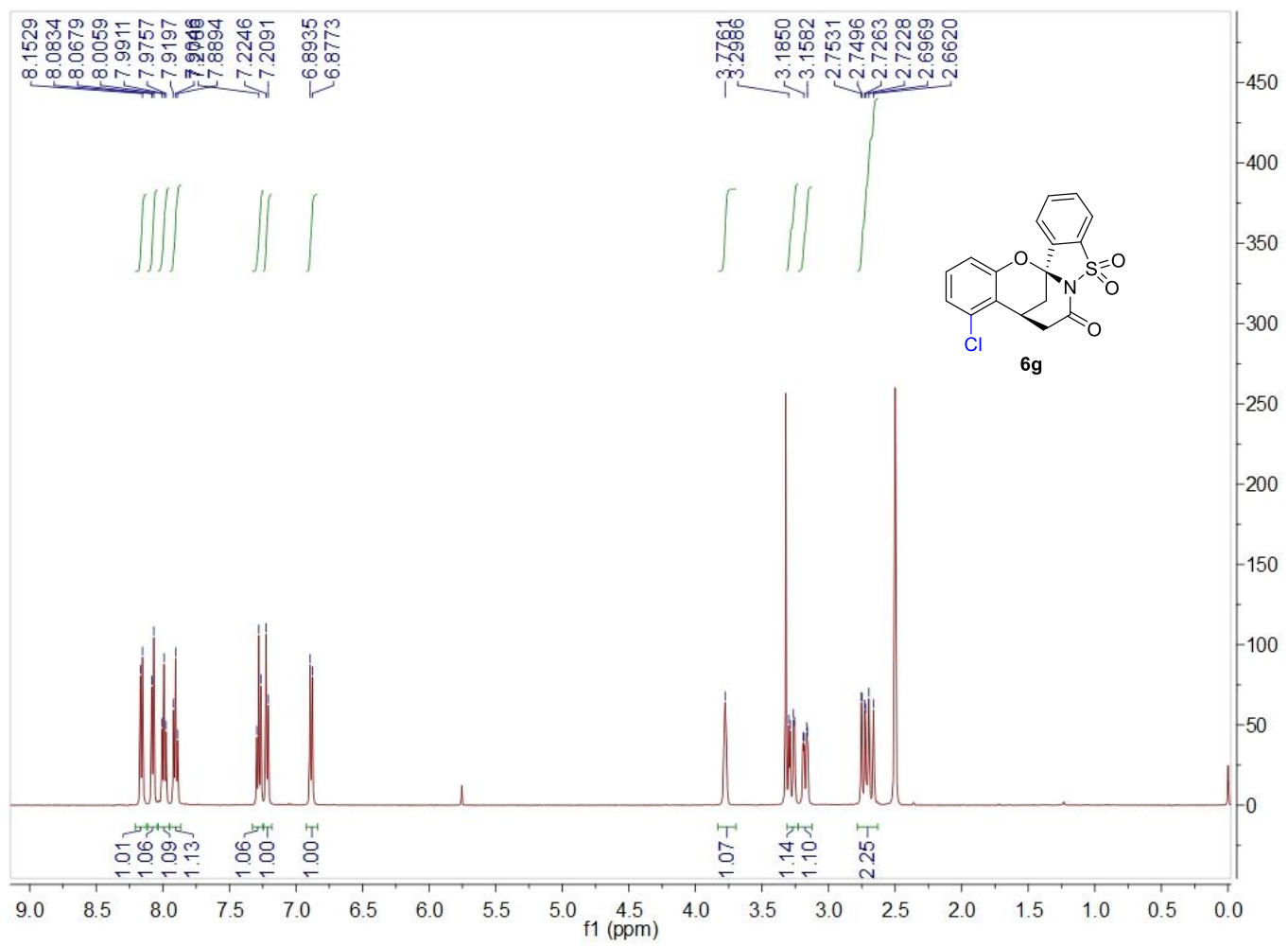


${ }^{13}$ C NMR of $6 g, d_{6}$-DMSO

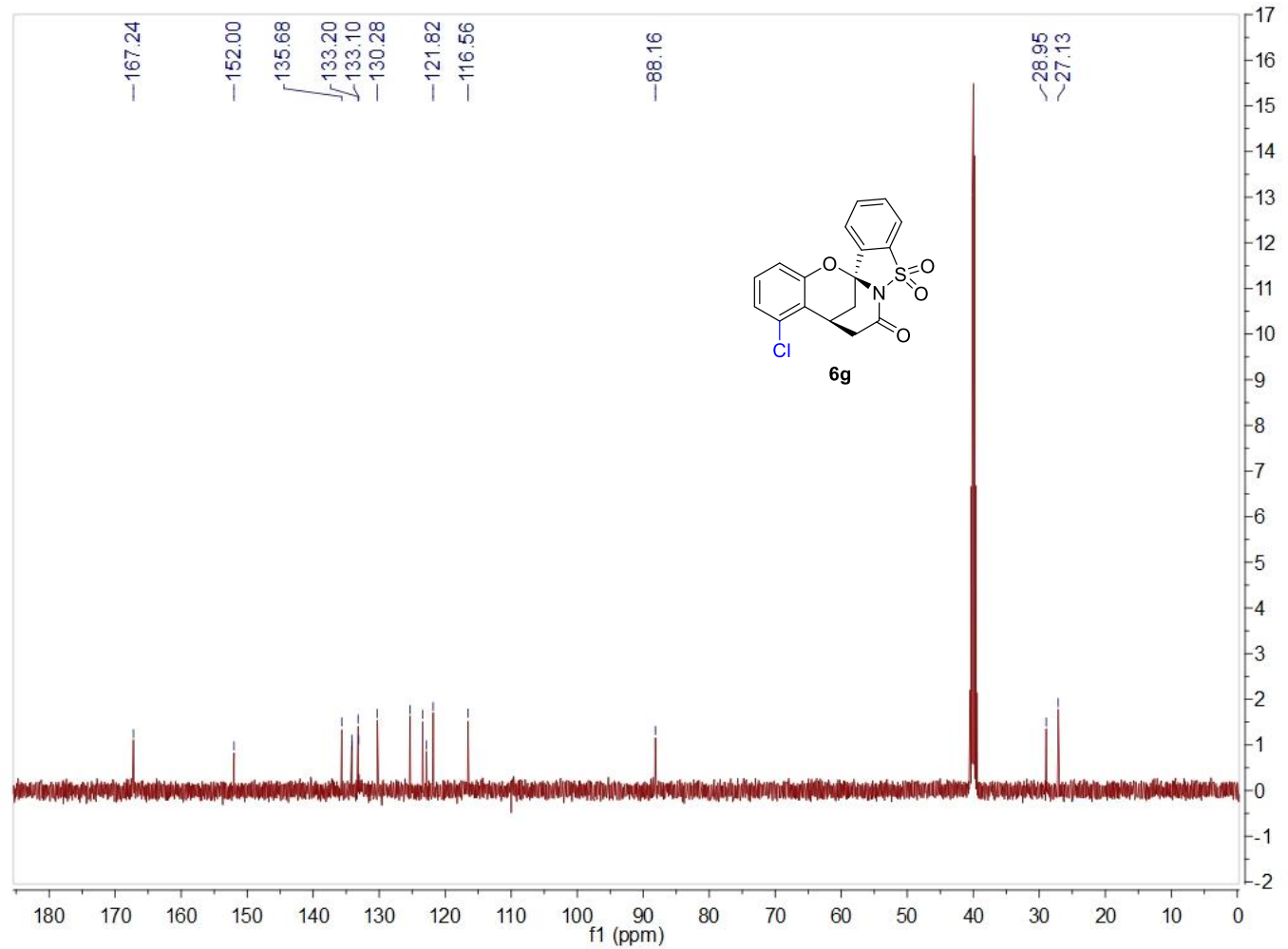

Chiral HPLC analysis of rac-6g

Chrom Type: Fixed WL Chromatogram, $210 \mathrm{~nm}$

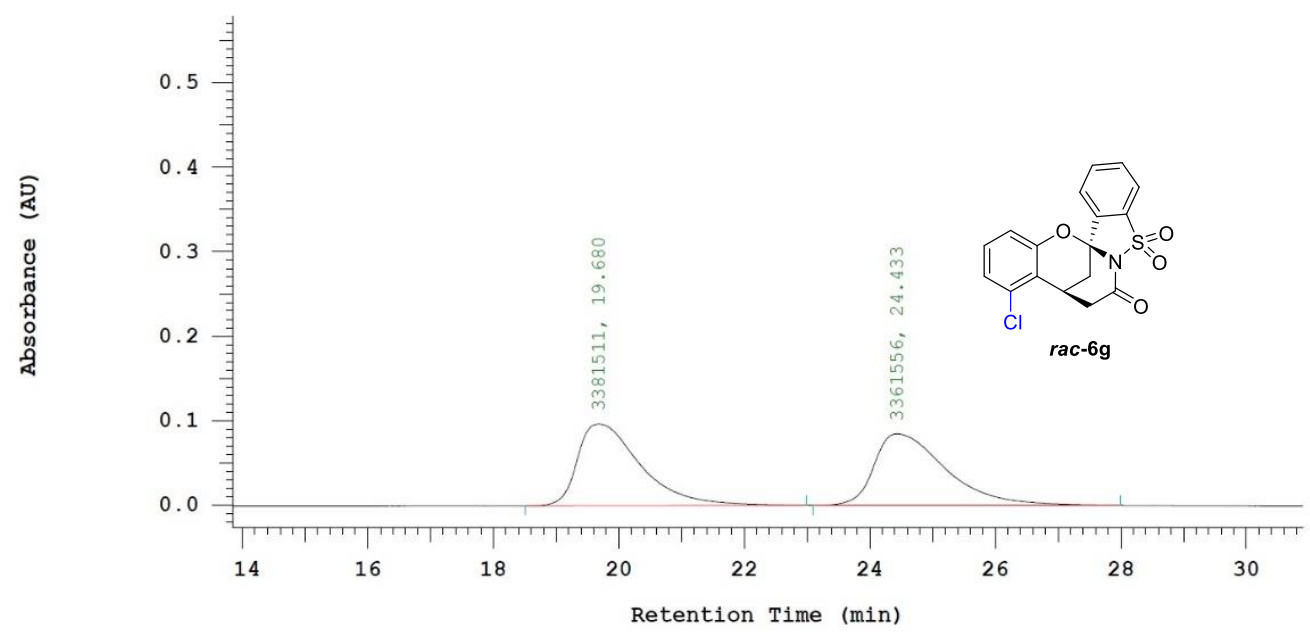

Chrom Type: Fixed WL Chromatogram, $210 \mathrm{~nm}$

Peak Quantitation: AREA

Calculation Method: AREA응

\begin{tabular}{ccccc} 
No. & RT & Area & Area \% & BC \\
\hline 1 & 19.680 & 3381511 & 50.148 & BB \\
2 & 24.433 & 3361556 & 49.852 & BB \\
\hline & & 6743067 & 100.000
\end{tabular}




\section{Chiral HPLC analysis of $\mathbf{6 g}$}

Chrom Type: Fixed WL Chromatogram, $210 \mathrm{~nm}$

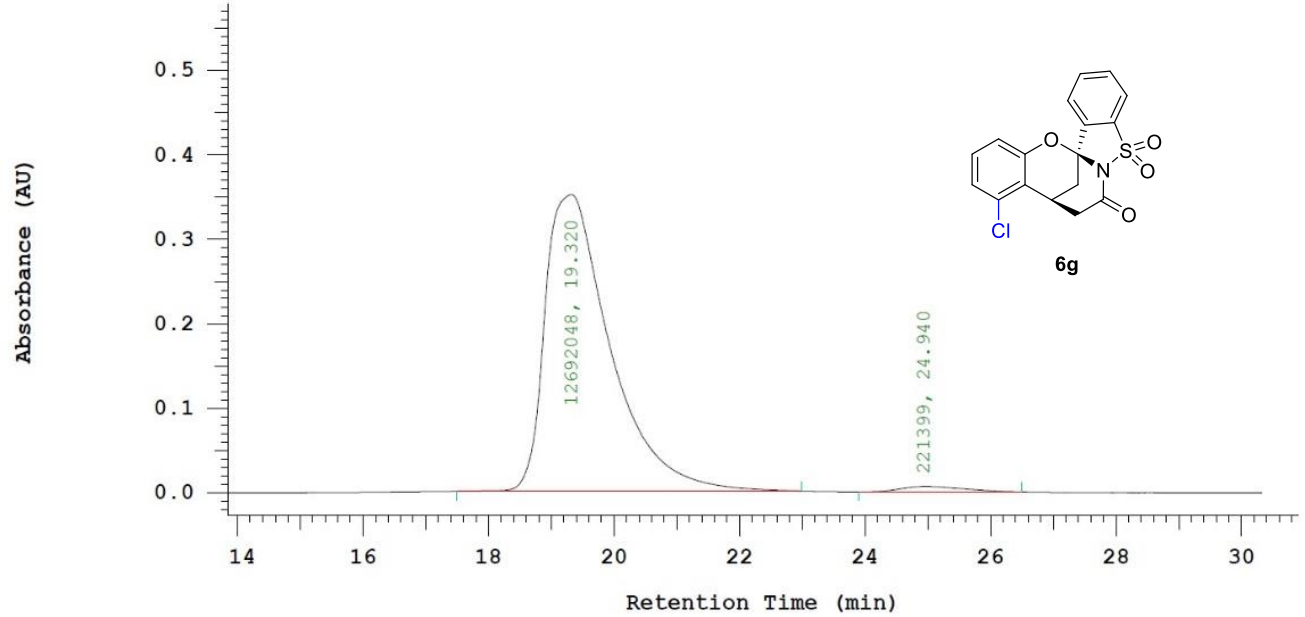

Chrom Type: Fixed WL Chromatogram, $210 \mathrm{~nm}$

Peak Quantitation: AREA

Calculation Method: AREA음

\begin{tabular}{|c|c|c|c|}
\hline No. & $\mathrm{RT}$ & Area & Area 응 \\
\hline 1 & 19.320 & 12692048 & 98.286 \\
\hline 2 & 24.940 & 221399 & 1.714 \\
\hline & & 12913447 & 100.000 \\
\hline
\end{tabular}

${ }^{1} \mathrm{H} \mathrm{NMR}$ of $8 \mathrm{a}, \mathrm{CDCl}_{3}$

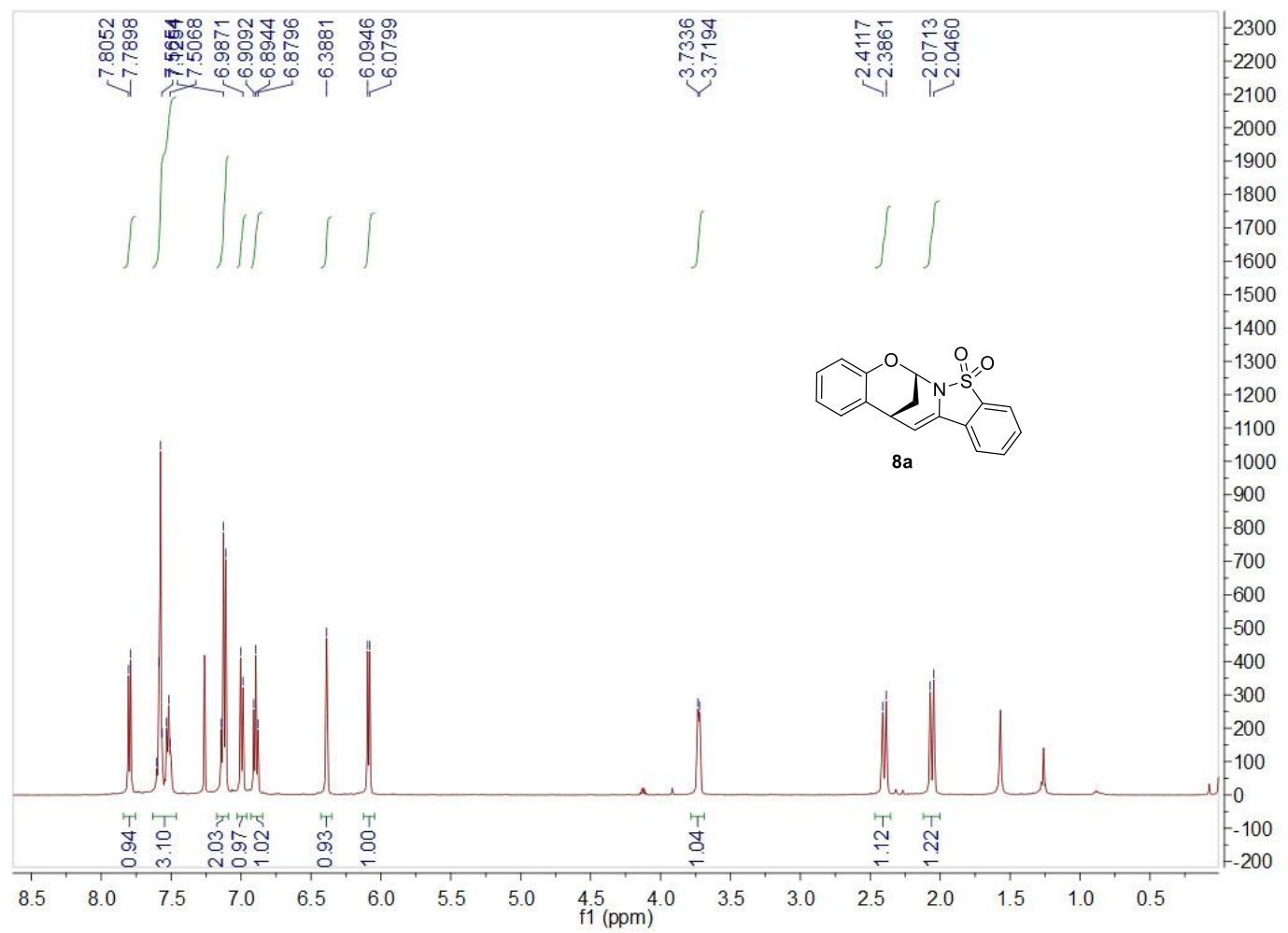


${ }^{13} \mathrm{C} \mathrm{NMR}$ of $8 \mathrm{a}, \mathrm{CDCl}_{3}$

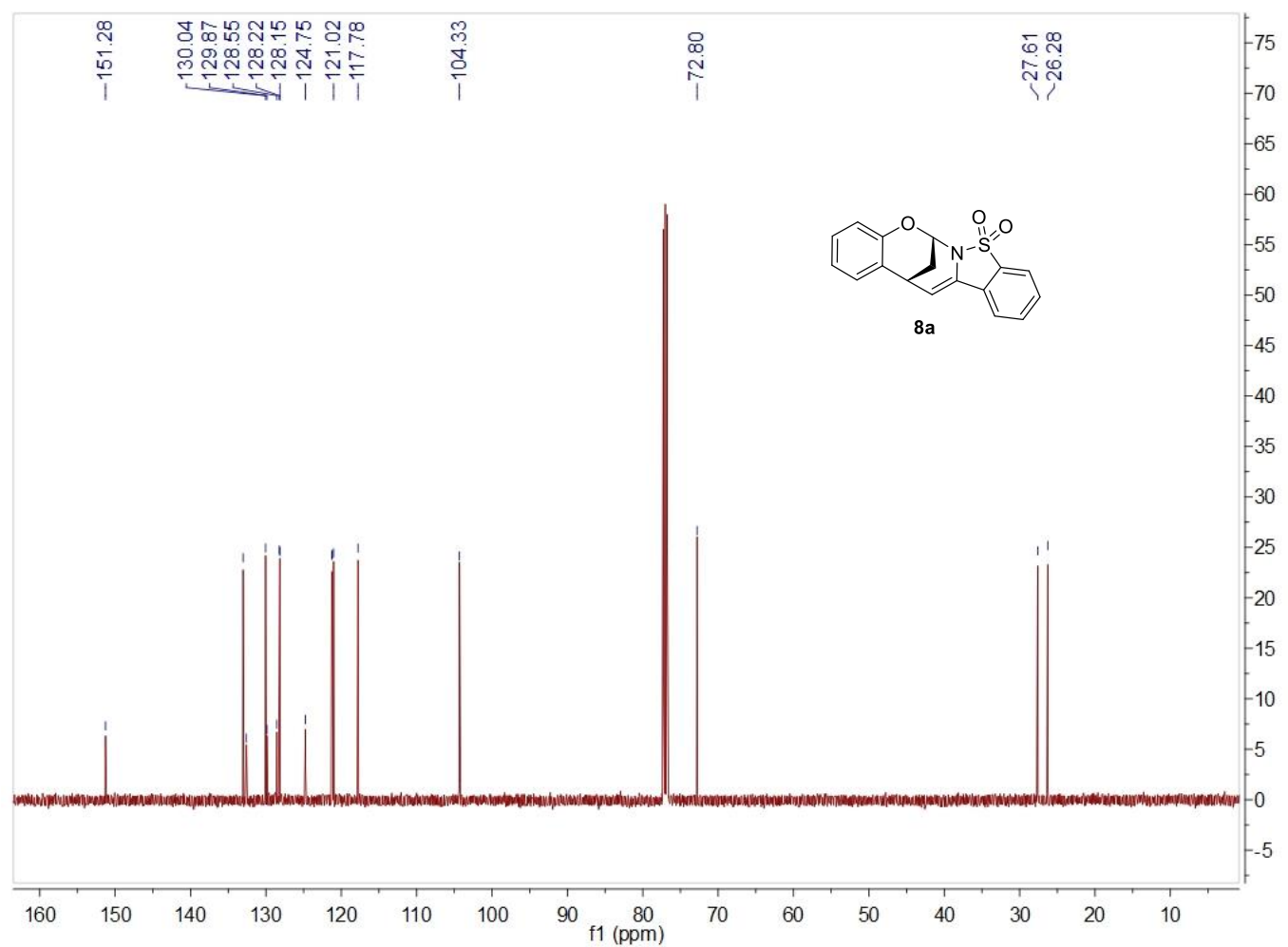

${ }^{1} \mathrm{H}-{ }^{1} \mathrm{H}$ COSY of $8 \mathrm{a}, \mathrm{CDCl}_{3}$

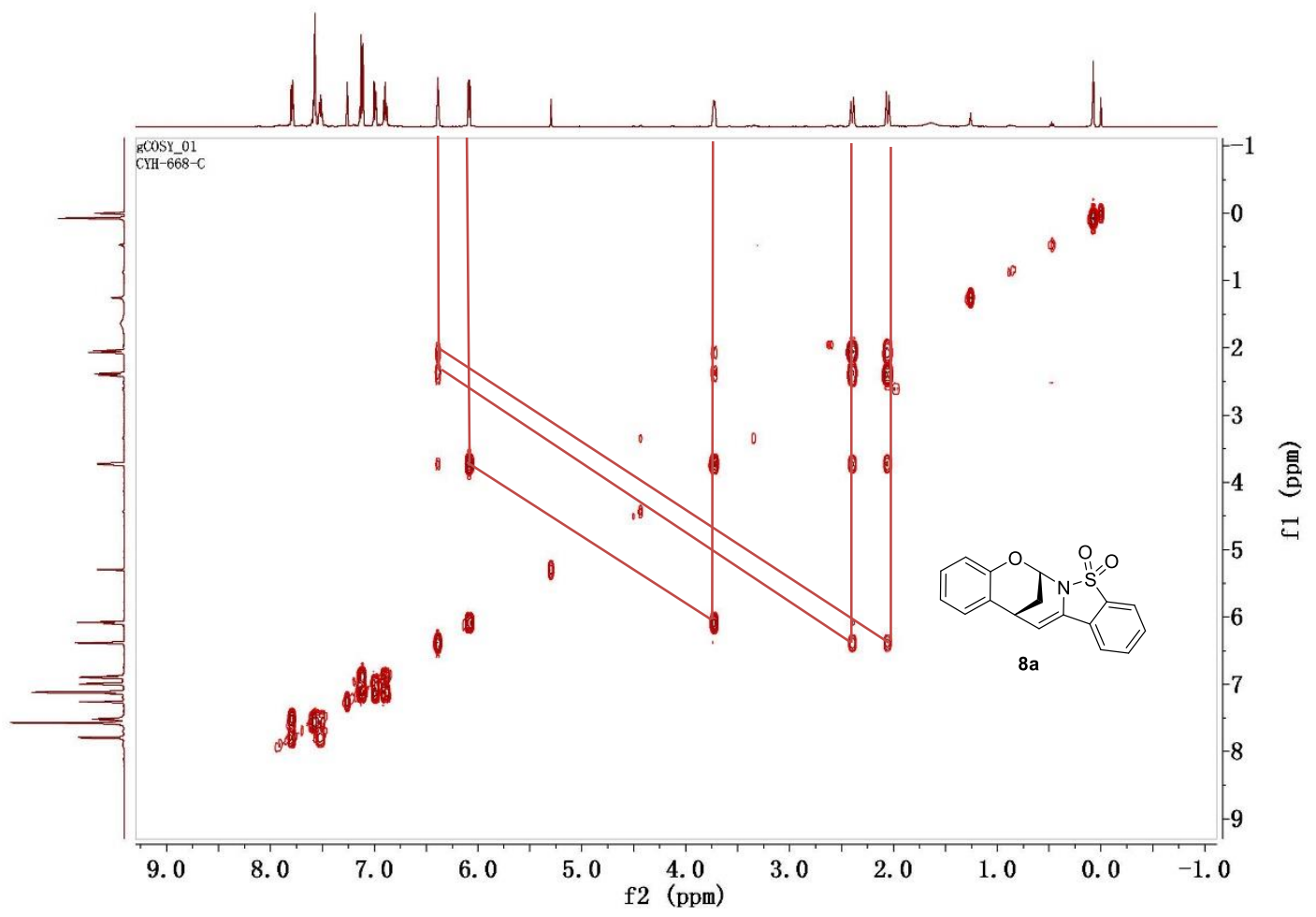




\section{Chiral HPLC analysis of rac-8a}

Chrom Type: Fixed WL Chromatogram, 216 nm

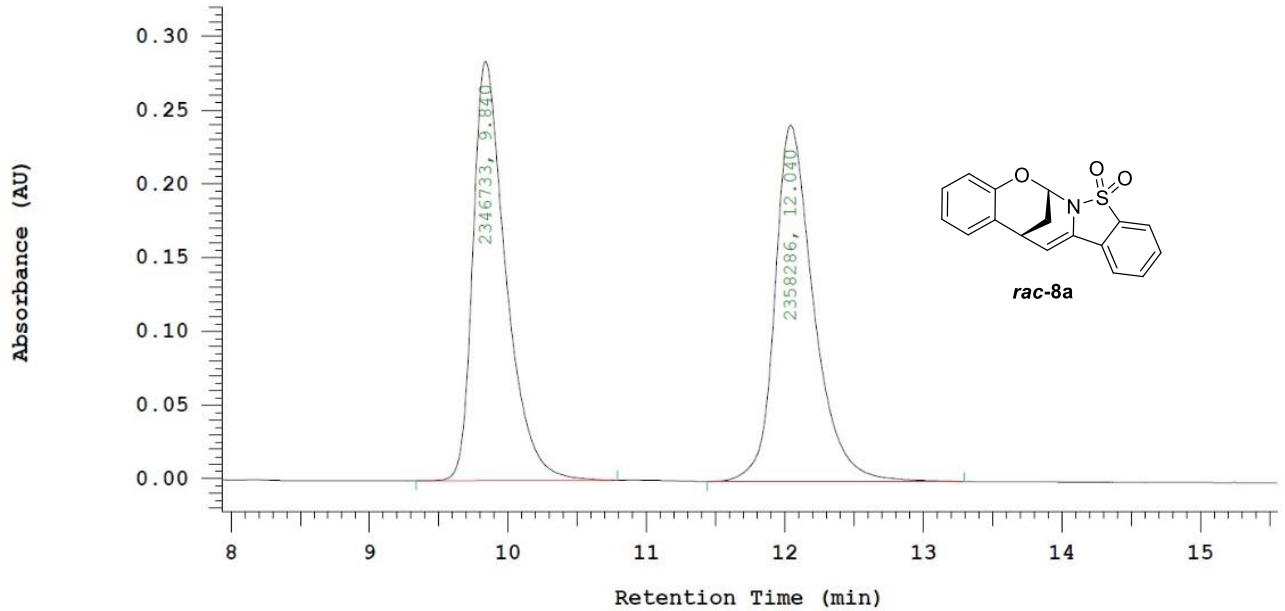

Chrom Type: Fixed WL Chromatogram, 216 nm

Peak Quantitation: AREA

Calculation Method: AREA응

\begin{tabular}{rrrrr}
\multicolumn{1}{c}{ RT } & Area & Area $\%$ & BC \\
\hline 1 & 9.840 & 2346733 & 49.877 & BB \\
2 & 12.040 & 2358286 & 50.123 & BB \\
\hline & & 4705019 & 100.000
\end{tabular}

\section{Chiral HPLC analysis of $\mathbf{8 a}$}

Chrom Type: Fixed WL Chromatogram, 216 nm

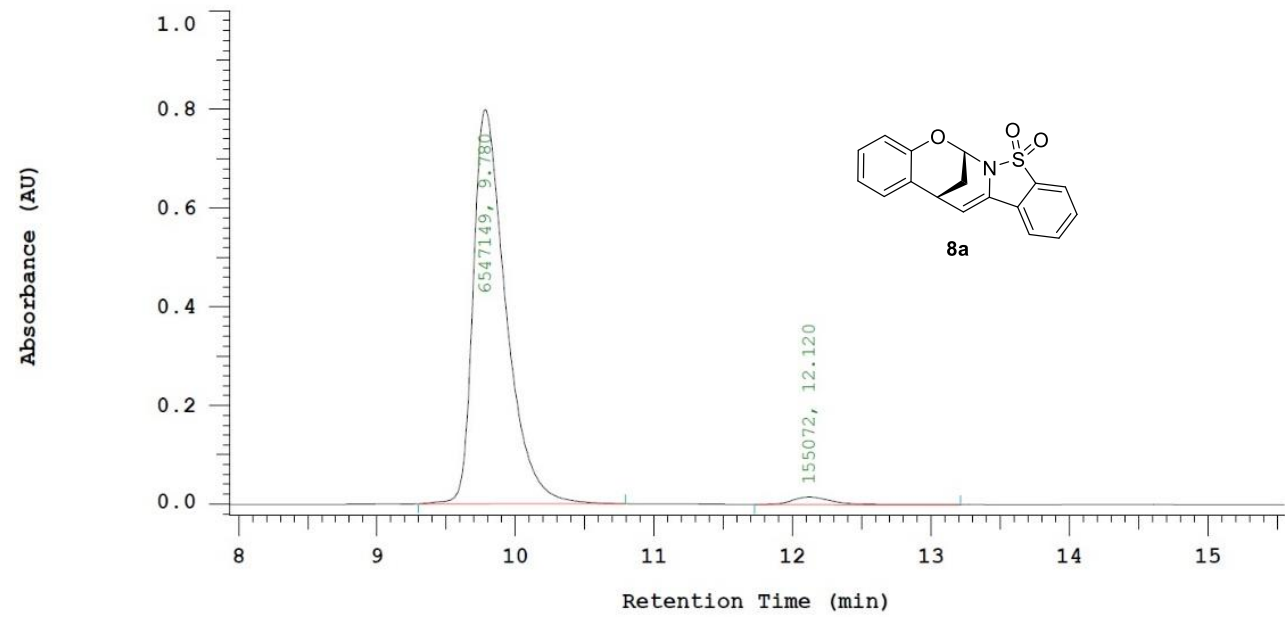

Chrom Type: Fixed WL Chromatogram, 216 nm

Peak Quantitation: AREA

Calculation Method: AREA응

\begin{tabular}{rrrrr}
\multicolumn{1}{c}{ RT } & Area & Area & BC \\
\hline 1 & 9.780 & 6547149 & 97.686 & BB \\
12.120 & 155072 & 2.314 & BB \\
\hline & & 6702221 & 100.000
\end{tabular}




\section{Chiral HPLC analysis of $8 \mathrm{a}(1 \mathrm{mmol})$}

Chrom Type: Fixed WL Chromatogram, $225 \mathrm{~nm}$

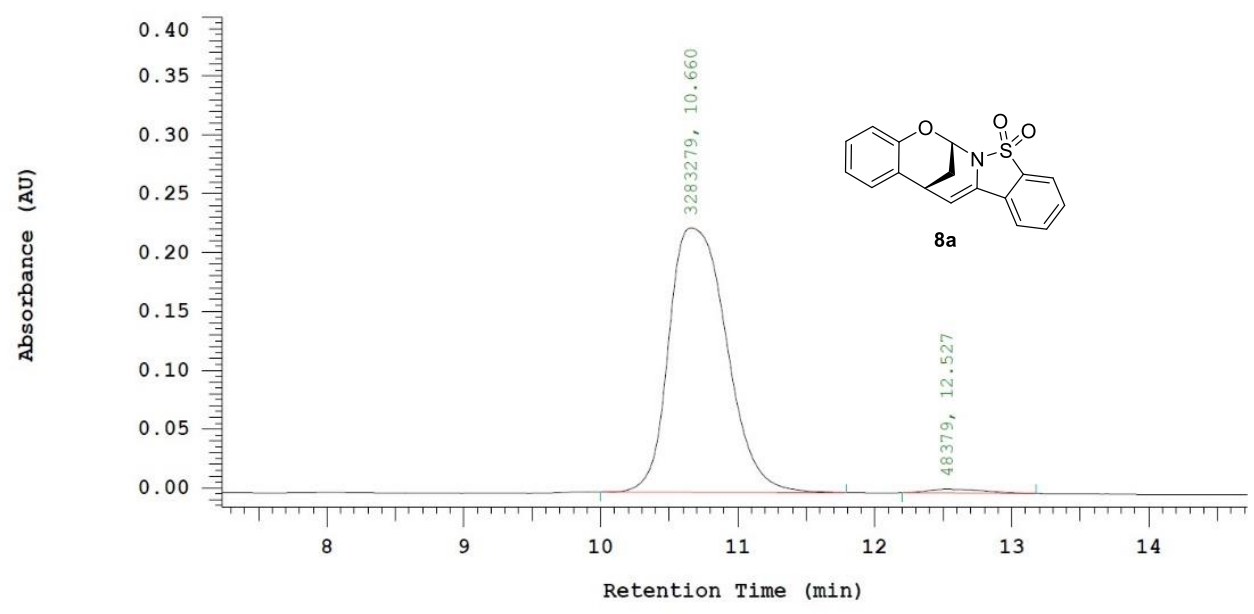

Chrom Type: Fixed WL Chromatogram, $225 \mathrm{~nm}$

Peak Quantitation: AREA

Calculation Method: AREA\%

\begin{tabular}{|c|c|c|c|c|}
\hline Jo. & $\mathrm{RT}$ & Area & Area 음 & $\mathrm{BC}$ \\
\hline 1 & 10.660 & 3283279 & 98.548 & BB \\
\hline 2 & 12.527 & 48379 & 1.452 & BB \\
\hline & & 3331658 & 100.000 & \\
\hline
\end{tabular}

\section{${ }^{1} \mathrm{H}$ NMR of $\mathbf{8 b}, \mathrm{CDCl}_{3}$}

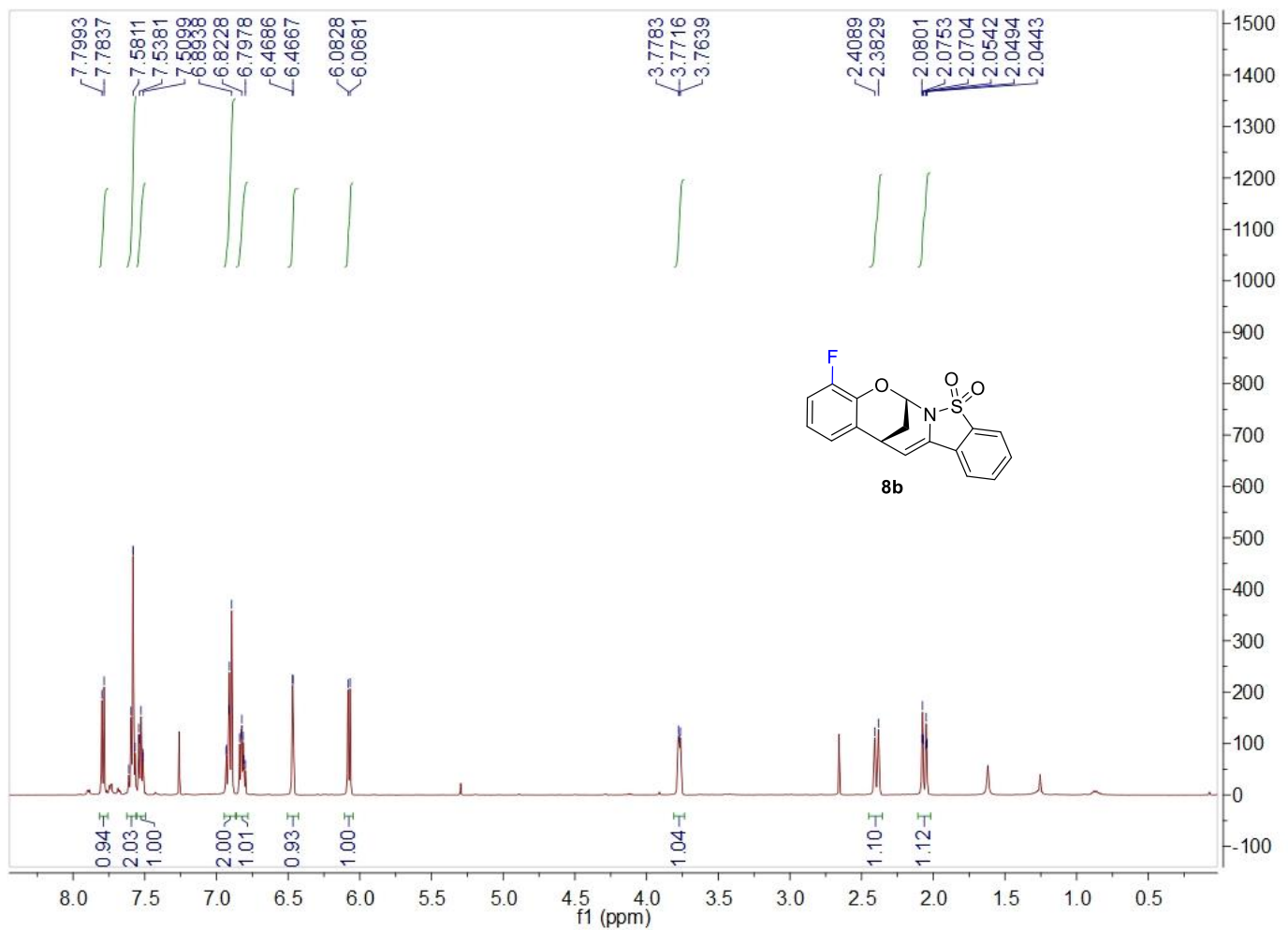


${ }^{13} \mathrm{C} \mathrm{NMR}$ of $\mathbf{8 b}, \mathrm{CDCl}_{3}$

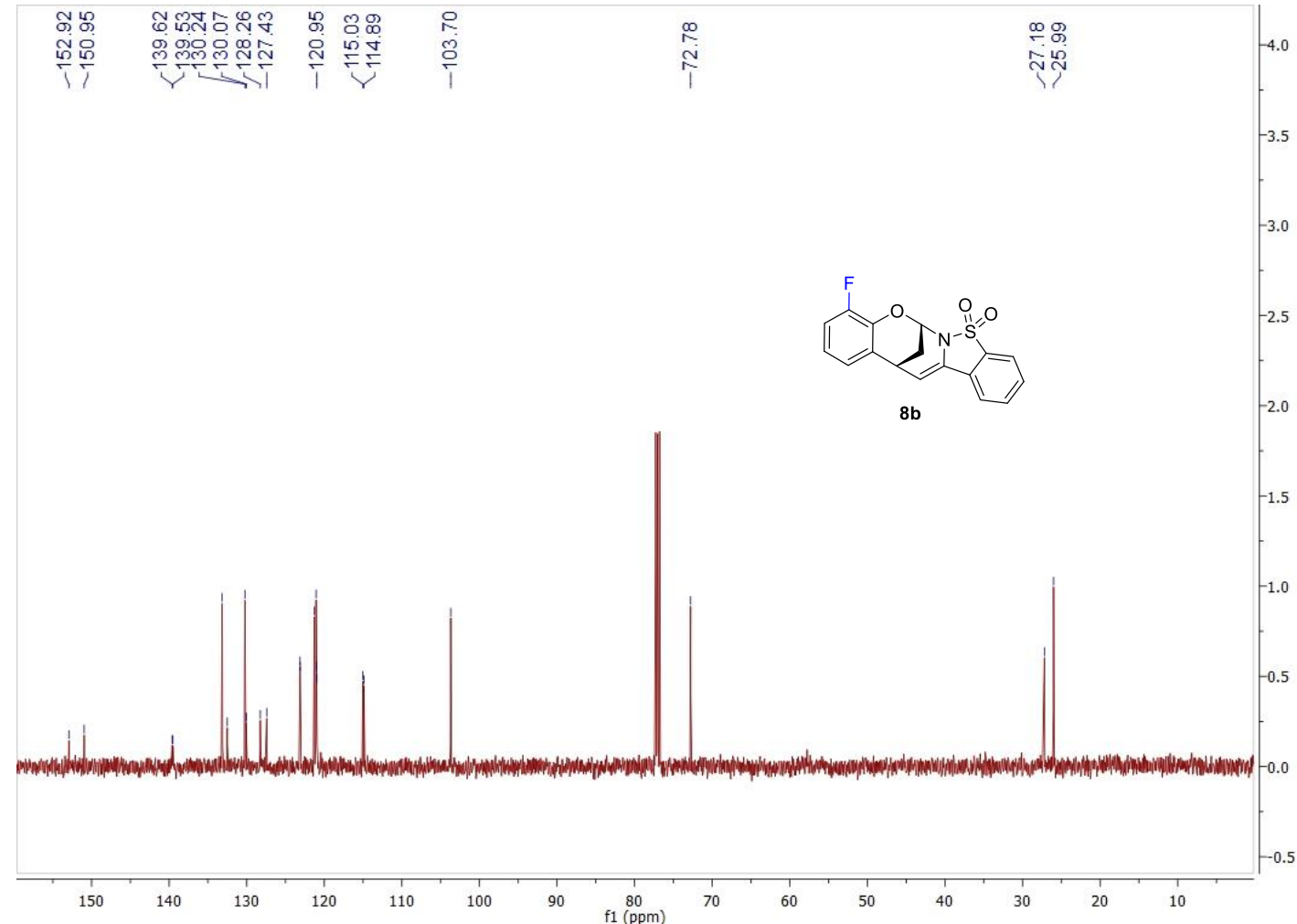

Chiral HPLC analysis of rac-8b

Chrom Type: Fixed WL Chromatogram, 216 nm

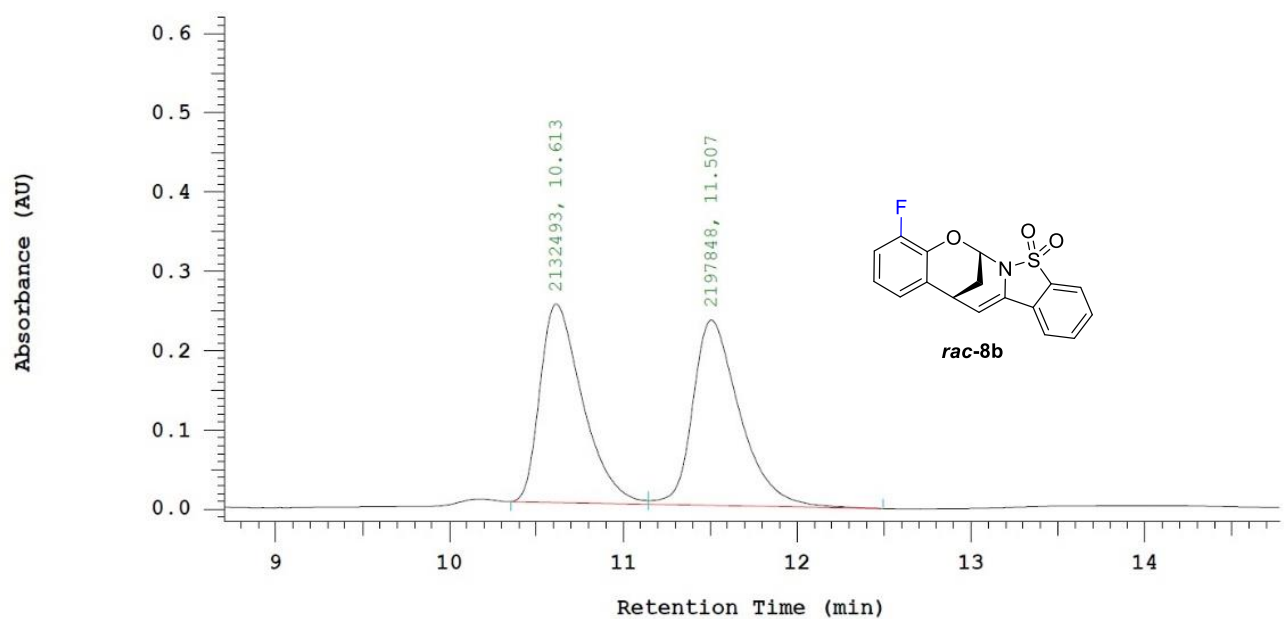

Chrom Type: Fixed WL Chromatogram, $216 \mathrm{~nm}$

Peak Quantitation: AREA

Calculation Method: AREA응

\begin{tabular}{ccccc} 
No. & RT & Area & Area & BC \\
\hline 1 & 10.613 & 2132493 & 49.245 & BV \\
2 & 11.507 & 2197848 & 50.755 & VB \\
\hline & & 4330341 & 100.000
\end{tabular}




\section{Chiral HPLC analysis of $\mathbf{8 b}$}

Chrom Type: Fixed WL Chromatogram, 216 nm

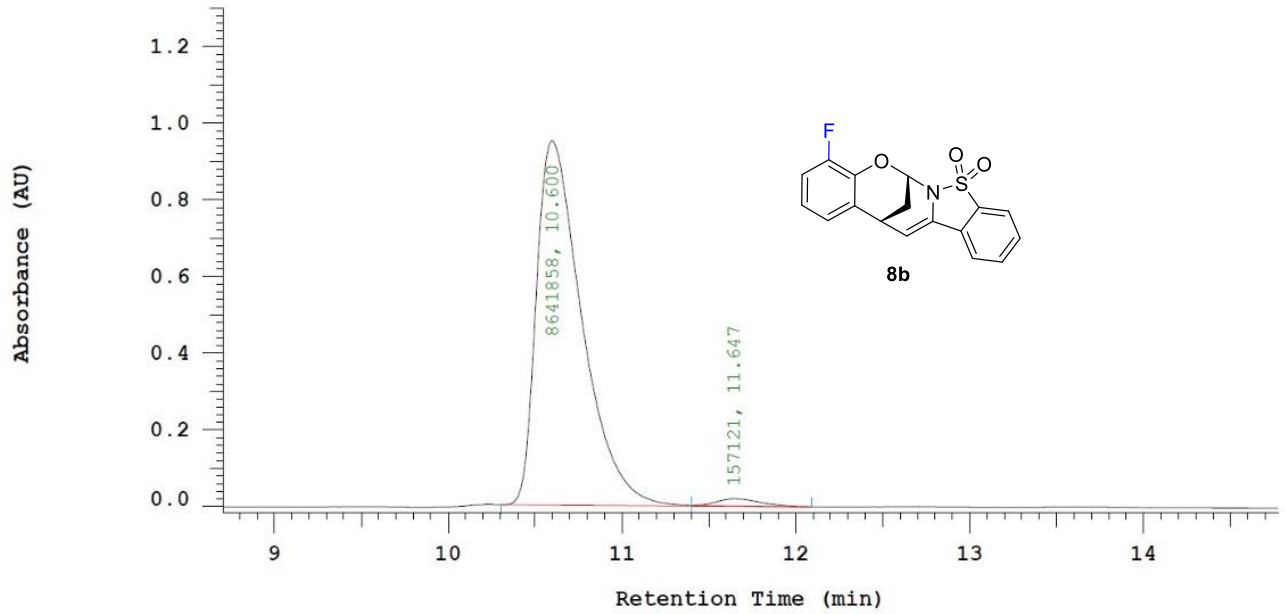

Chrom Type: Fixed WL Chromatogram, $216 \mathrm{~nm}$

Peak Quantitation: AREA

Calculation Method: AREA응

\begin{tabular}{ccrrr} 
No. & RT & Area & Area & BC \\
\hline 1 & 10.600 & 8641858 & 98.214 & BV \\
2 & 11.647 & 157121 & 1.786 & TBB \\
\hline & & 8798979 & 100.000 &
\end{tabular}

${ }^{1} \mathrm{H} \mathrm{NMR}$ of $8 \mathrm{c}, \mathrm{CDCl}_{3}$

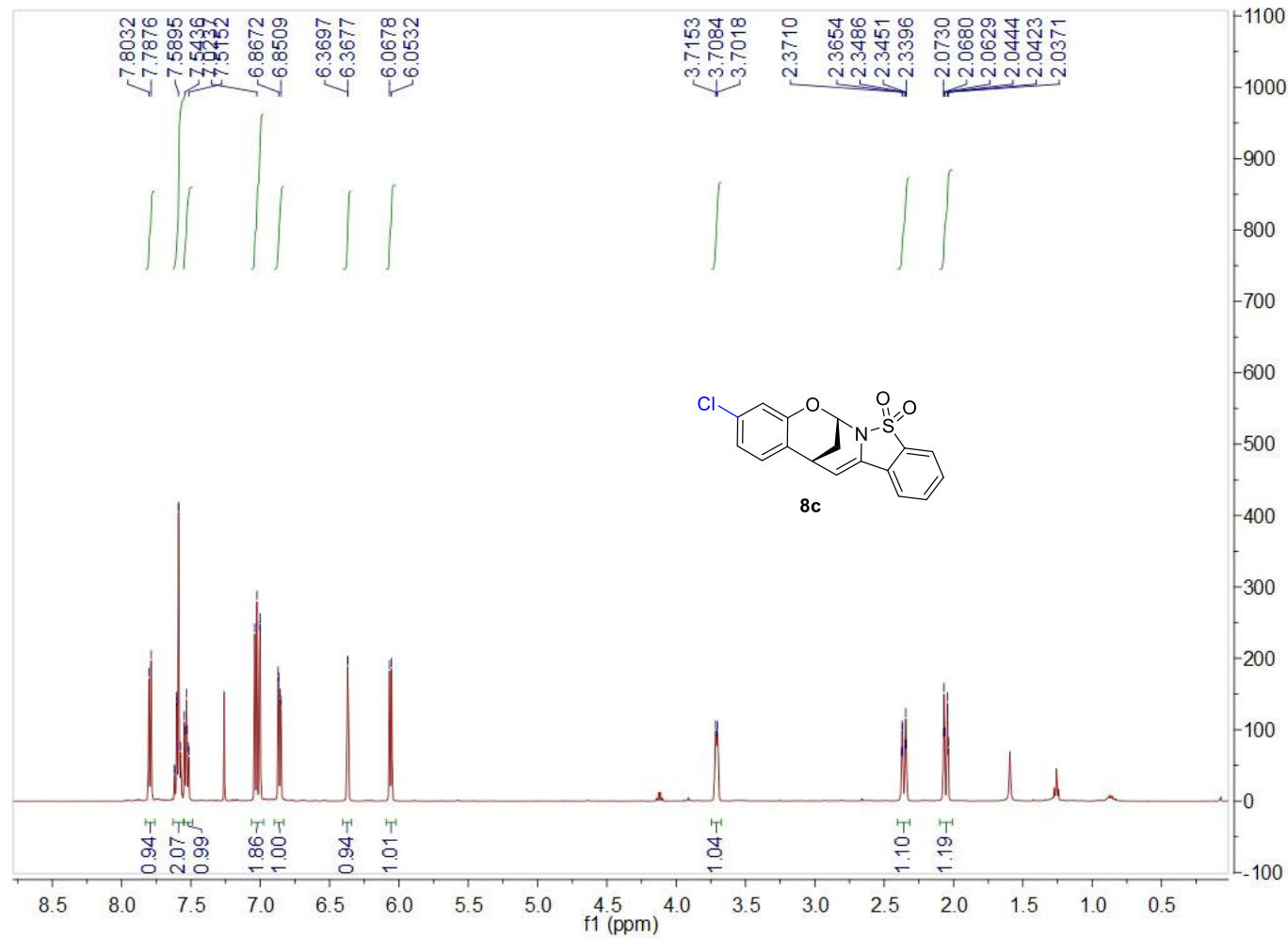


${ }^{13} \mathrm{C}$ NMR of $8 \mathrm{c}, \mathrm{CDCl}_{3}$

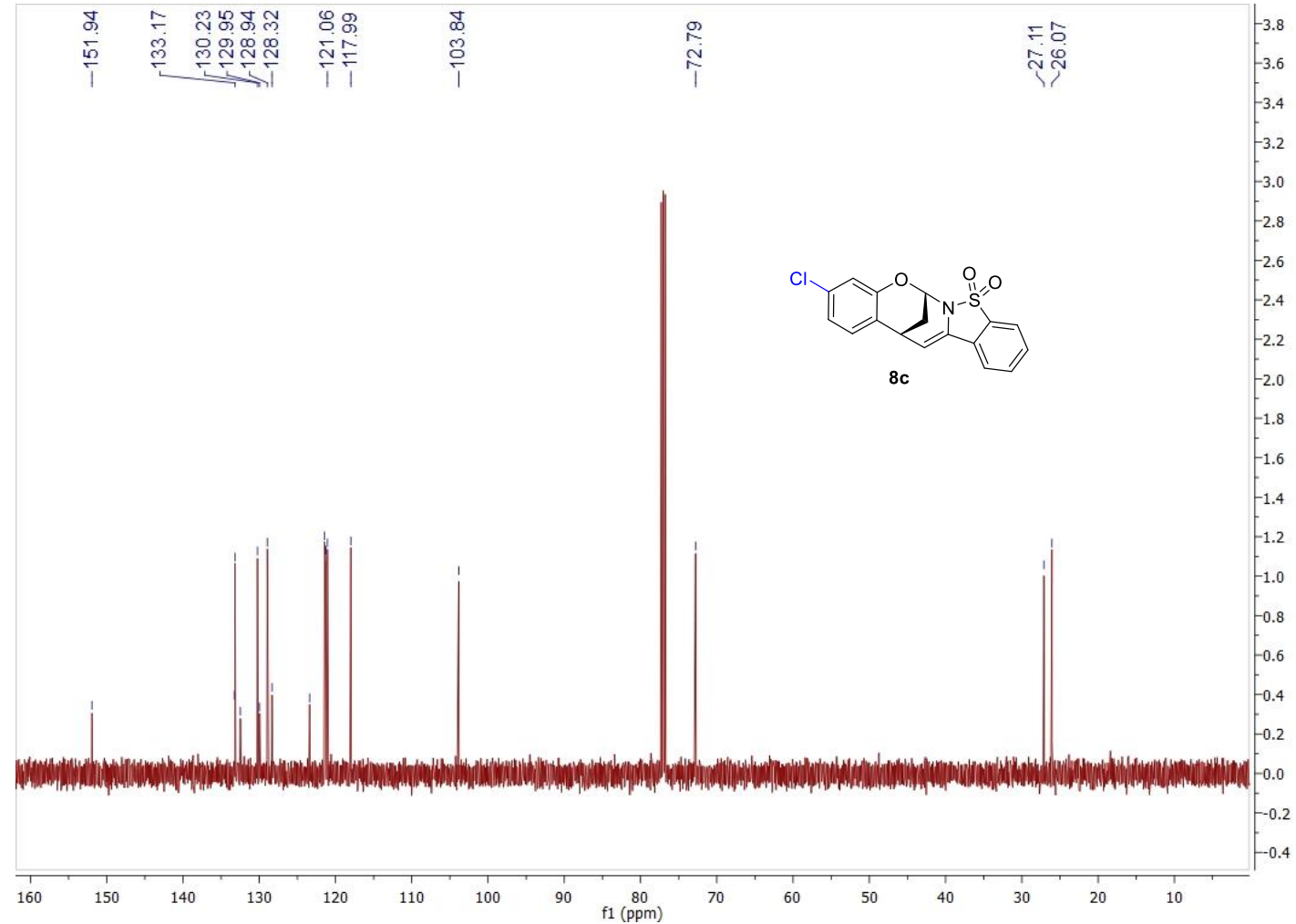

Chiral HPLC analysis of rac-8c

Chrom Type: Fixed WL Chromatogram, $216 \mathrm{~nm}$

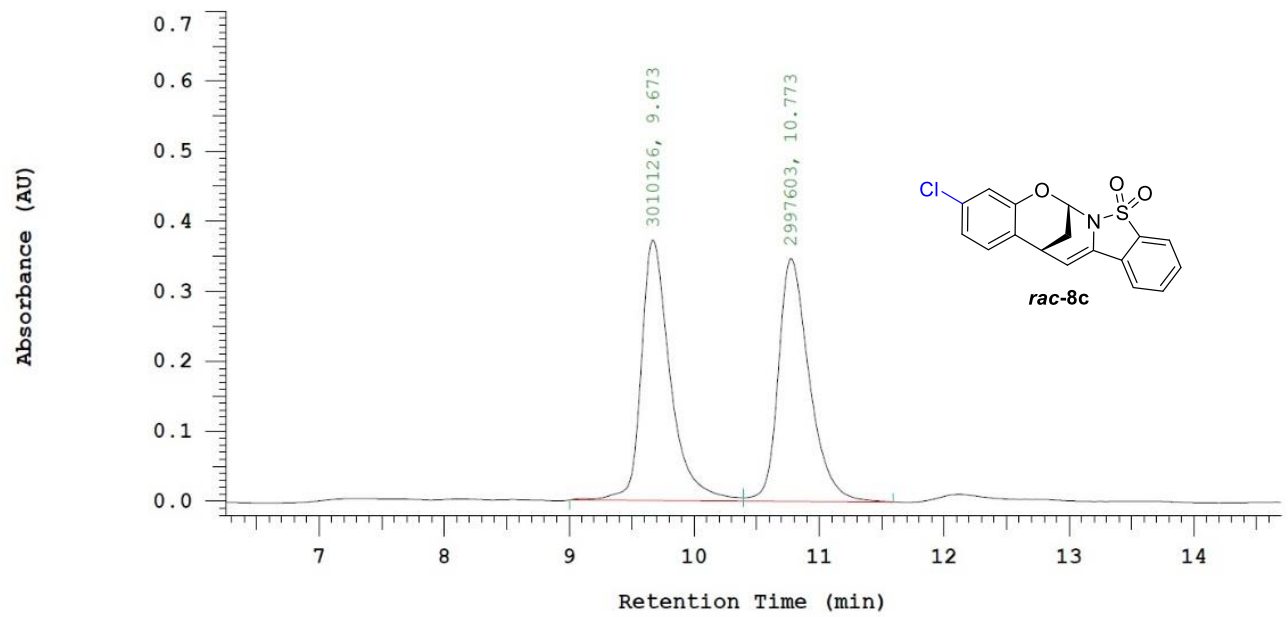

Chrom Type: Fixed WL Chromatogram, $216 \mathrm{~nm}$ Peak Quantitation: AREA

Calculation Method: AREA응

\begin{tabular}{rrrrr} 
No. & RT & Area & Area o & BC \\
\hline 1 & 9.673 & 3010126 & 50.104 & BV \\
2 & 10.773 & 2997603 & 49.896 & VB \\
\hline & & 6007729 & 100.000 & \\
\hline
\end{tabular}




\section{Chiral HPLC analysis of 8c}

Chrom Type: Fixed WL Chromatogram, $216 \mathrm{~nm}$

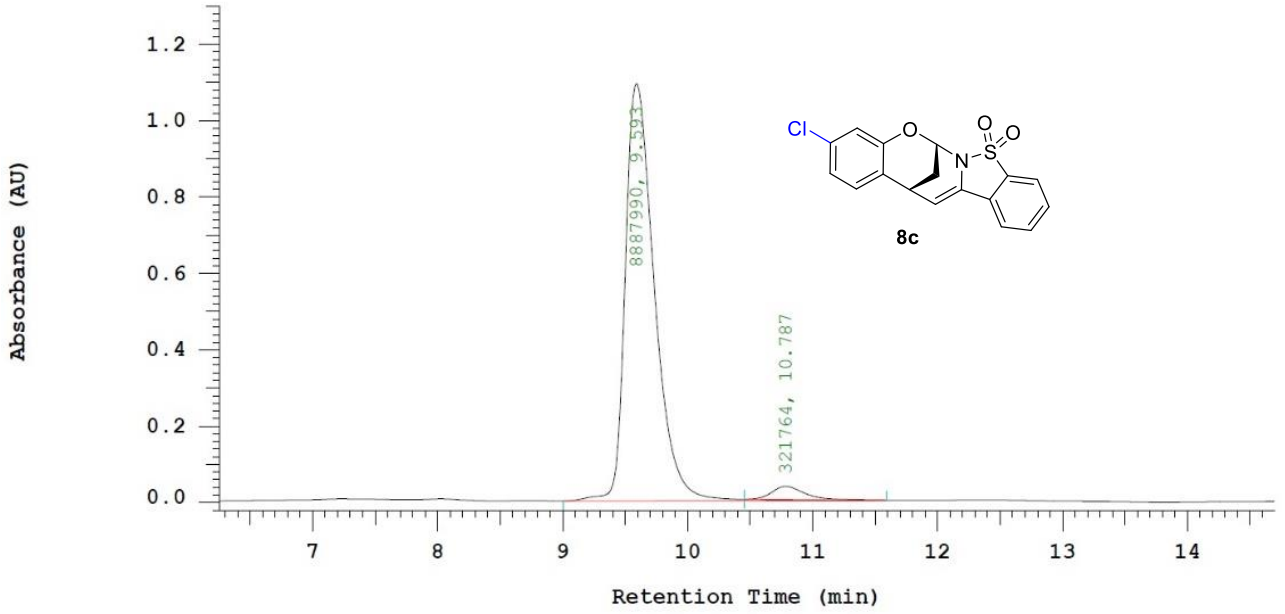

Chrom Type: Fixed WL Chromatogram, $216 \mathrm{~nm}$

Peak Quantitation: AREA

Calculation Method: AREA응

\begin{tabular}{rrrrr} 
No. & \multicolumn{1}{c}{ RT } & Area & Area $\%$ & BC \\
\hline 1 & 9.593 & 8887990 & 96.506 & BV \\
2 & 10.787 & 321764 & 3.494 & TBB \\
\hline & & 9209754 & 100.000
\end{tabular}

${ }^{1} \mathrm{H} \mathrm{NMR}$ of $8 \mathrm{~d}, \mathrm{CDCl}_{3}$

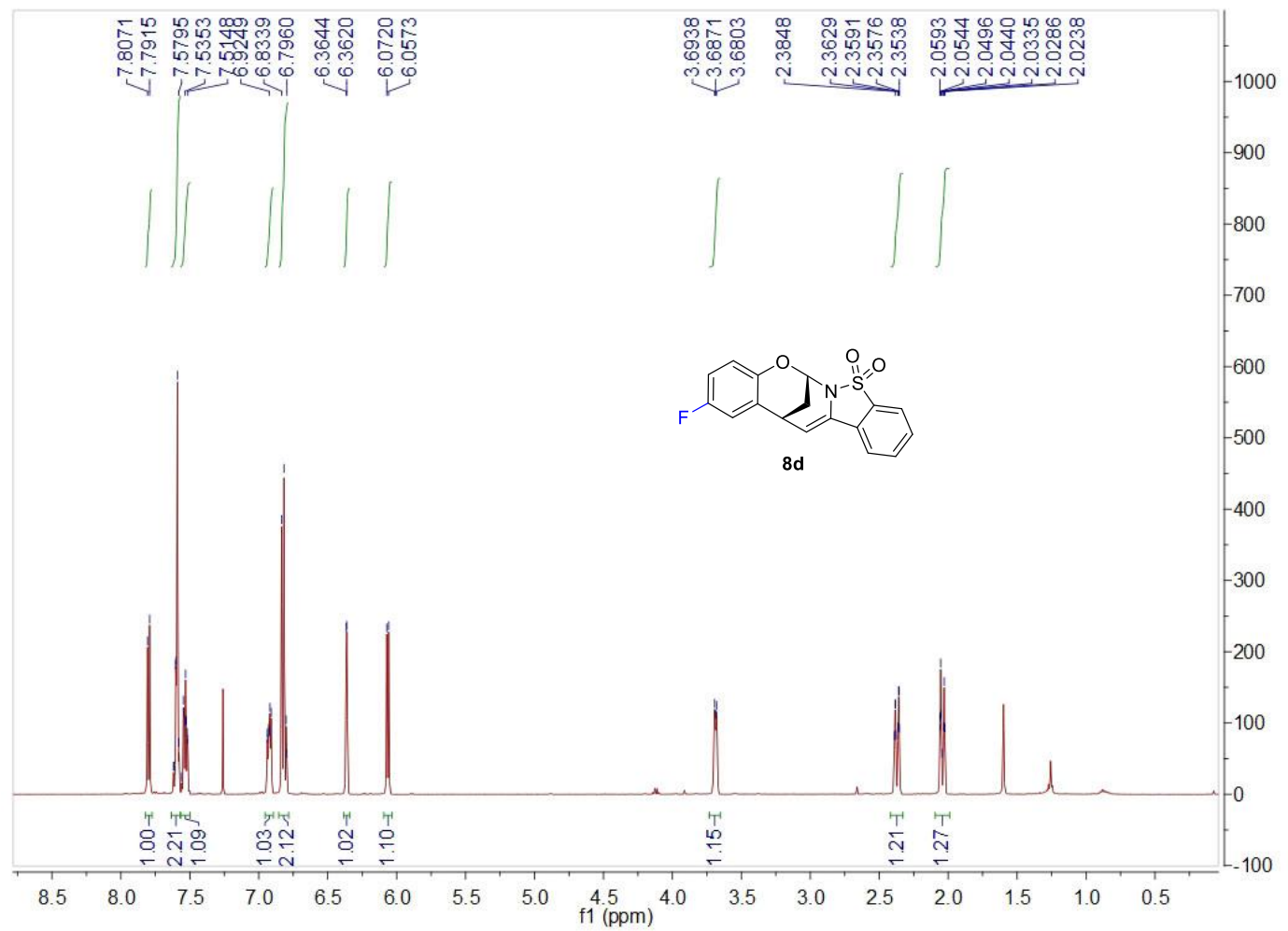


${ }^{13} \mathrm{C}$ NMR of $8 d, \mathrm{CDCl}_{3}$

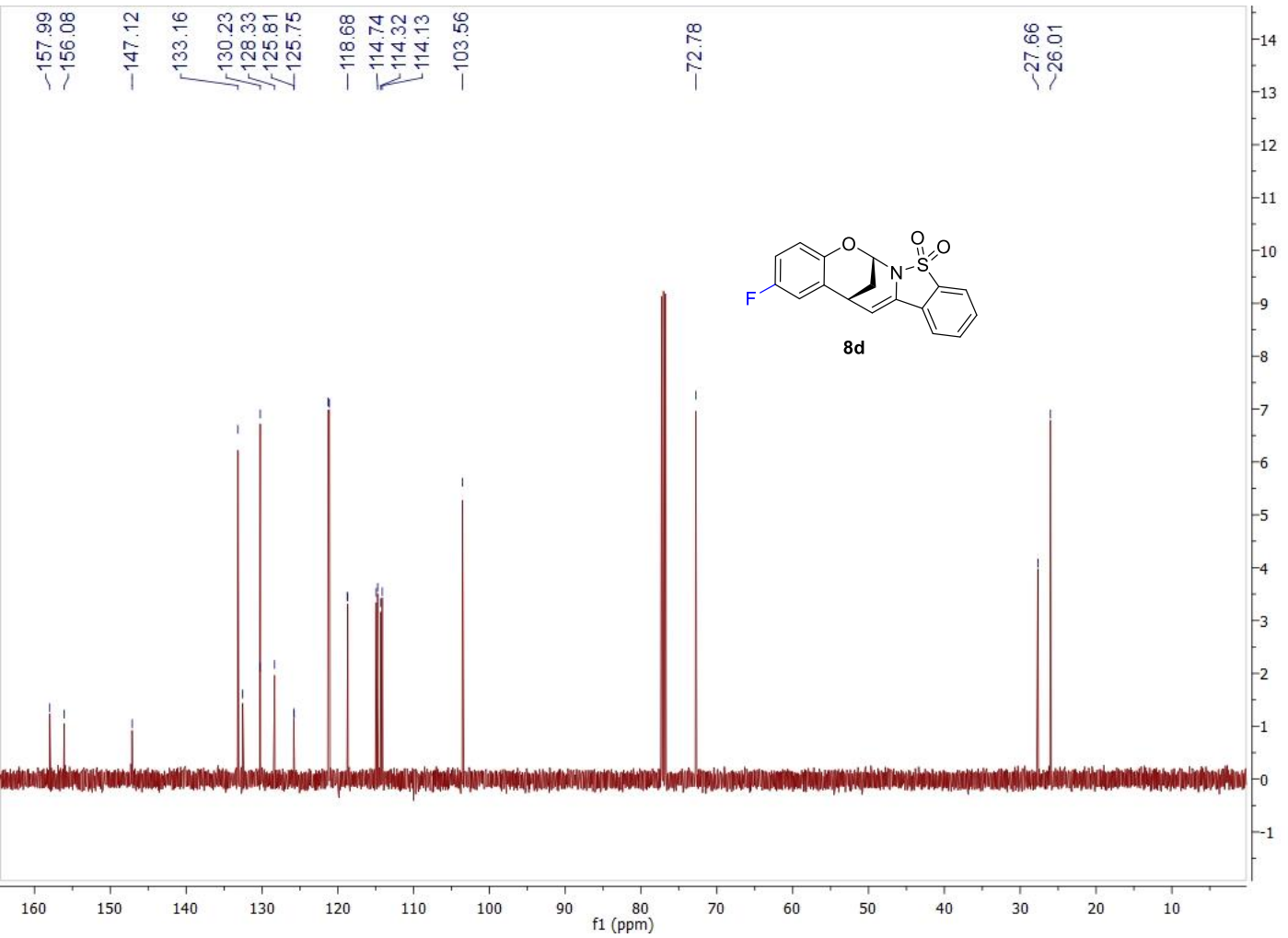

Chiral HPLC analysis of rac-8d

Chrom Type: Fixed WL Chromatogram, $216 \mathrm{~nm}$

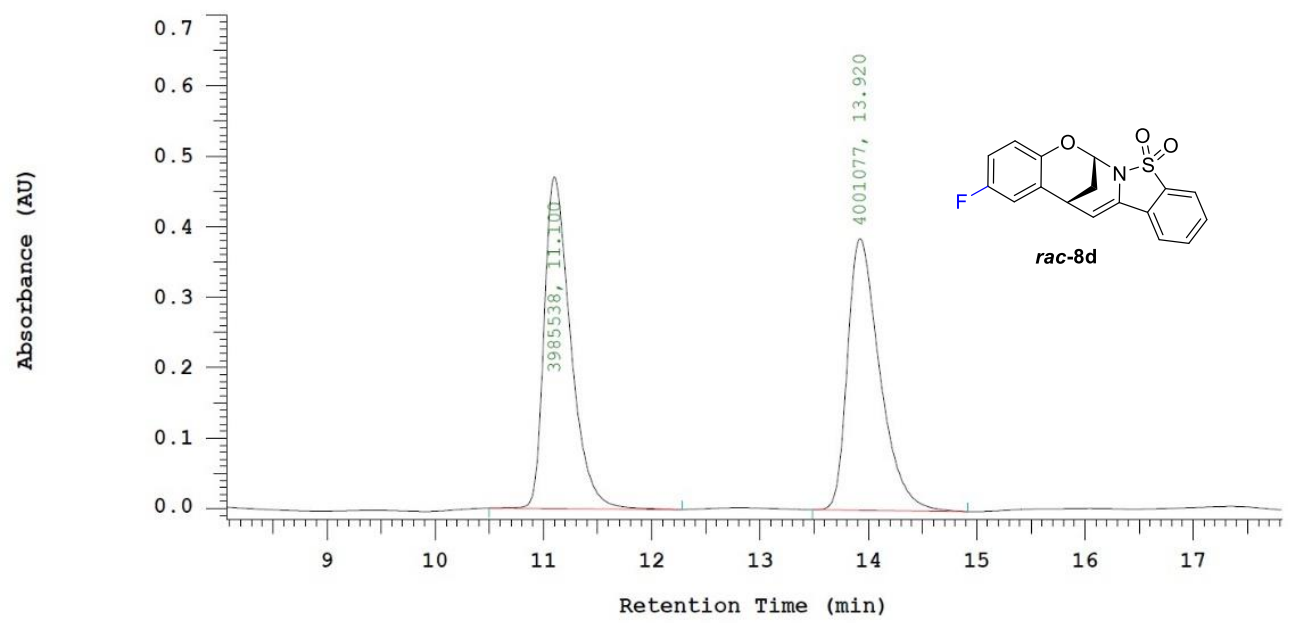

Chrom Type: Fixed WL Chromatogram, $216 \mathrm{~nm}$

Peak Quantitation: AREA

Calculation Method: AREA응

\begin{tabular}{ccccc} 
No. & RT & Area & Area 은 & BC \\
\hline 1 & 11.100 & 3985538 & 49.903 & BB \\
2 & 13.920 & 4001077 & 50.097 & BB \\
\hline & 7986615 & 100.000 & \\
\hline
\end{tabular}




\section{Chiral HPLC analysis of $\mathbf{8 d}$}

Chrom Type: Fixed WL Chromatogram, 216 nm

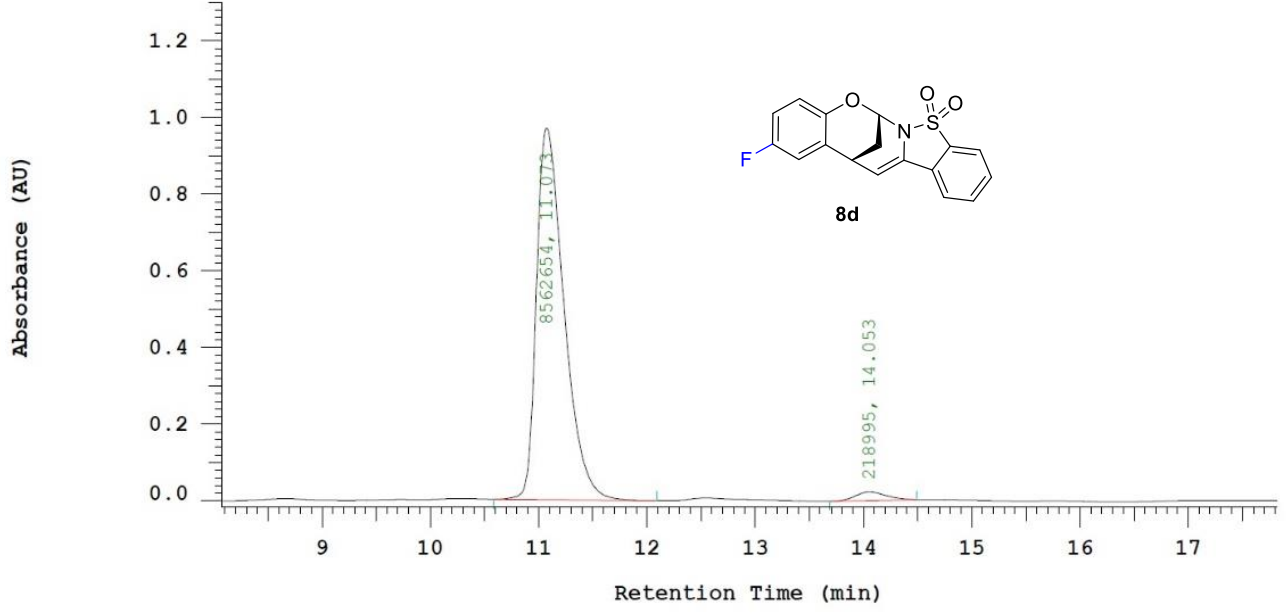

Chrom Type: Fixed WL Chromatogram, $216 \mathrm{~nm}$

Peak Quantitation: AREA

Calculation Method: AREA응

\begin{tabular}{|c|c|c|c|c|}
\hline No. & $\mathrm{RT}$ & Area & Area \% & $\mathrm{BC}$ \\
\hline 1 & 11.073 & 8562654 & 97.506 & $\mathrm{BB}$ \\
\hline 2 & 14.053 & 218995 & 2.494 & $\mathrm{BB}$ \\
\hline & & 8781649 & 100.000 & \\
\hline
\end{tabular}

${ }^{1} \mathrm{H} \mathrm{NMR}$ of $8 \mathbf{e}, \mathrm{CDCl}_{3}$

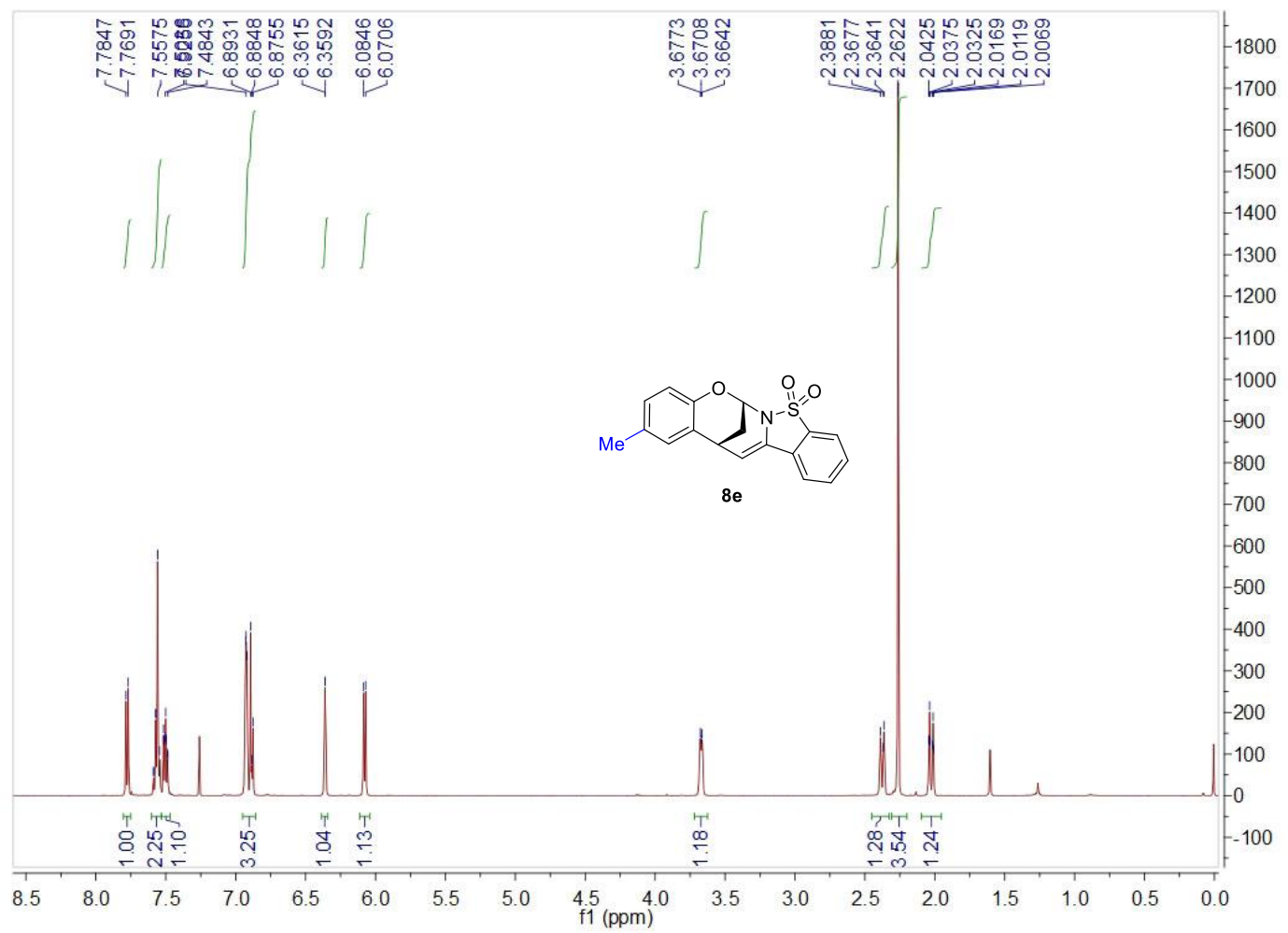


${ }^{13} \mathrm{CNMR}$ of $8 \mathrm{e}, \mathrm{CDCl}_{3}$

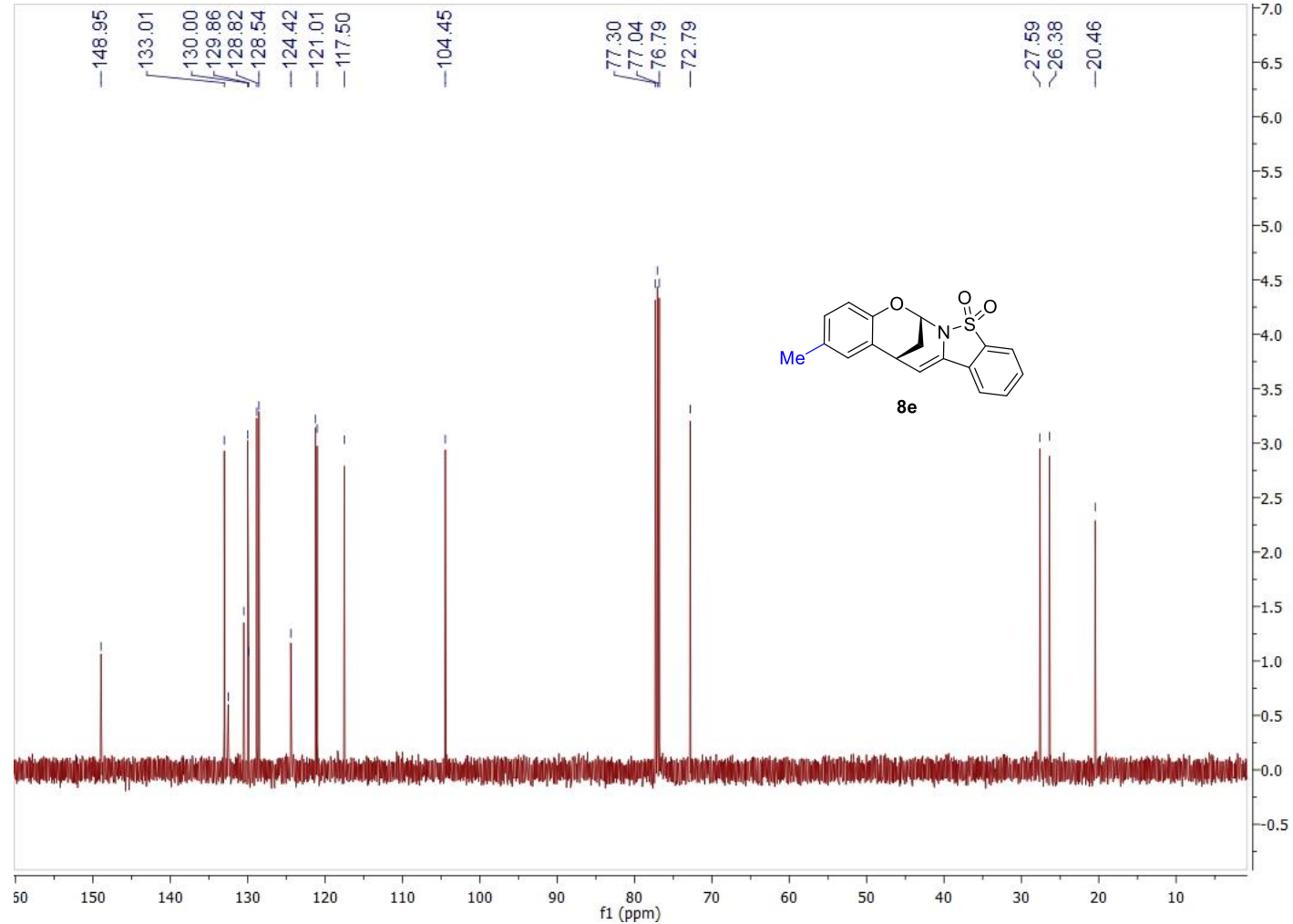

Chiral HPLC analysis of rac-8e

Chrom Type: Fixed WL Chromatogram, 216 nm

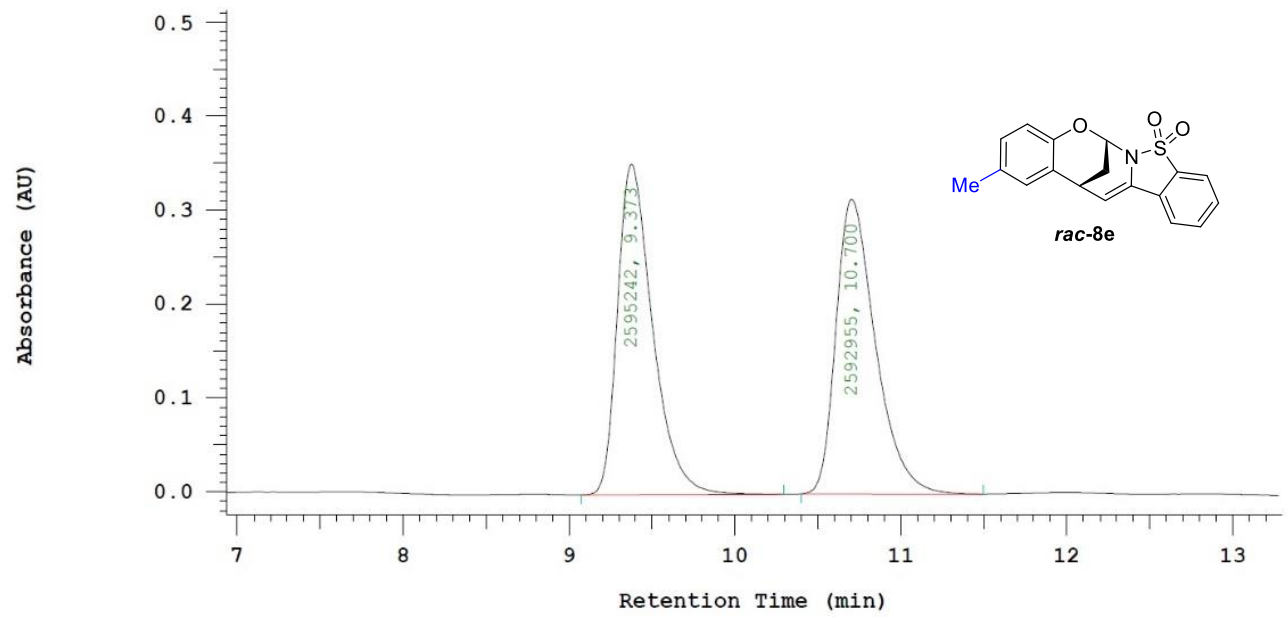

Chrom Type: Fixed WL Chromatogram, $216 \mathrm{~nm}$

Peak Quantitation: AREA

Calculation Method: AREA응

\begin{tabular}{rrrrr}
\multicolumn{1}{c}{ RT } & Area & Area & BC \\
\hline 1 & 9.373 & 2595242 & 50.022 & BB \\
2 & 10.700 & 2592955 & 49.978 & BB \\
\hline & & 5188197 & 100.000
\end{tabular}




\section{Chiral HPLC analysis of $\mathbf{8 e}$}

Chrom Type: Fixed WL Chromatogram, $216 \mathrm{~nm}$

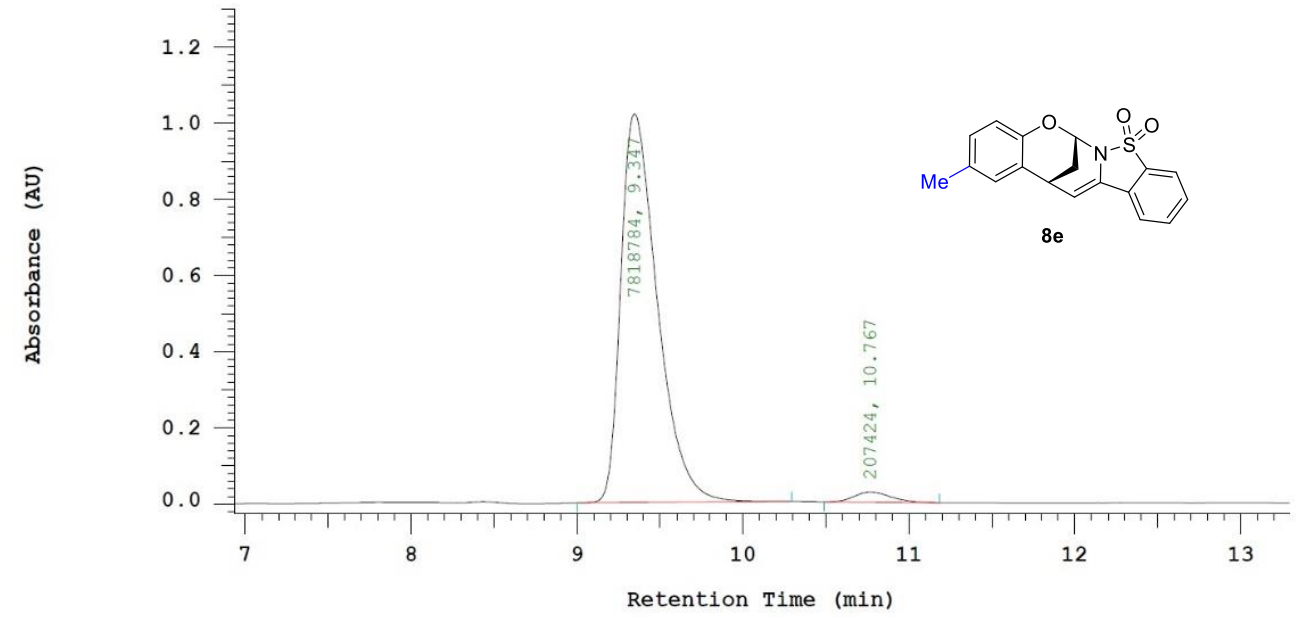

Chrom Type: Fixed WL Chromatogram, $216 \mathrm{~nm}$

Peak Quantitation: AREA

Calculation Method: AREA응

\begin{tabular}{|c|c|c|c|c|}
\hline No. & $\mathrm{RT}$ & Area & Area 응 & $\mathrm{BC}$ \\
\hline 1 & 9.347 & 7818784 & 97.416 & BB \\
\hline 2 & 10.767 & 207424 & 2.584 & BB \\
\hline & & 8026208 & 100.000 & \\
\hline
\end{tabular}

${ }^{1} \mathrm{H}$ NMR of $8 \mathrm{f}, \mathrm{CDCl}_{3}$

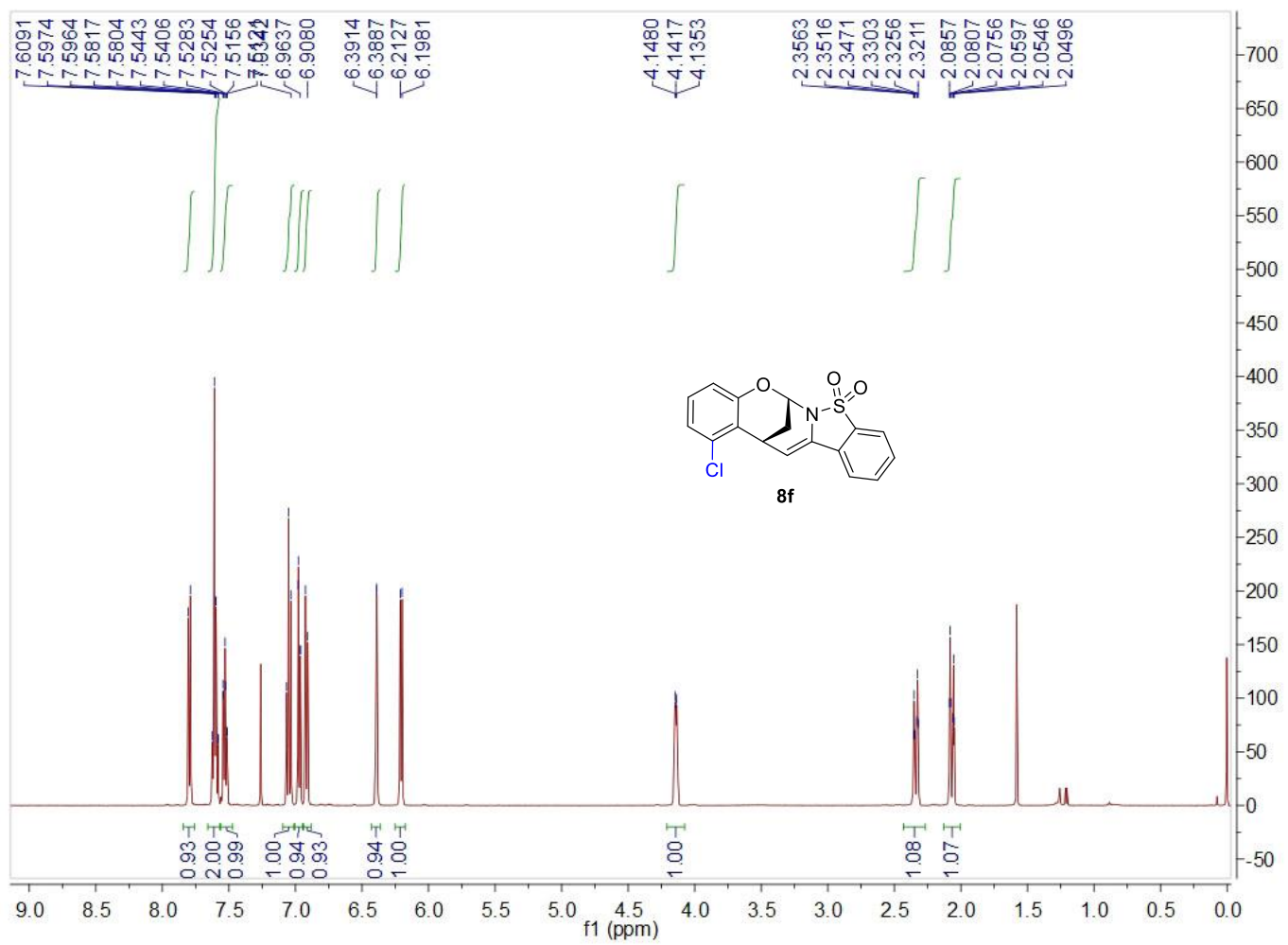


${ }^{13} \mathrm{C} \mathrm{NMR}$ of $8 \mathrm{f}, \mathrm{CDCl}_{3}$

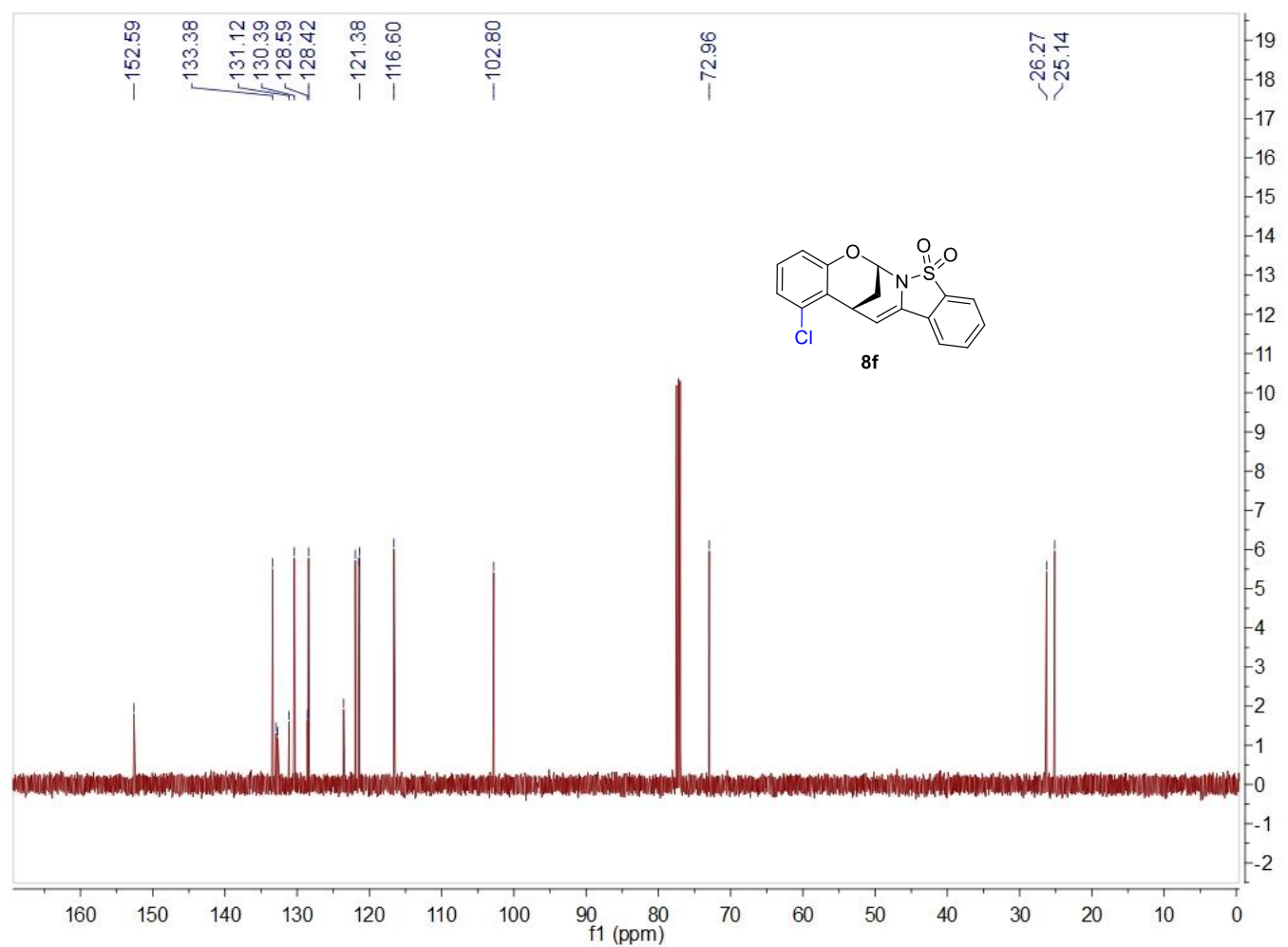

Chiral HPLC analysis of rac-8f

Chrom Type: Fixed WL Chromatogram, $210 \mathrm{~nm}$

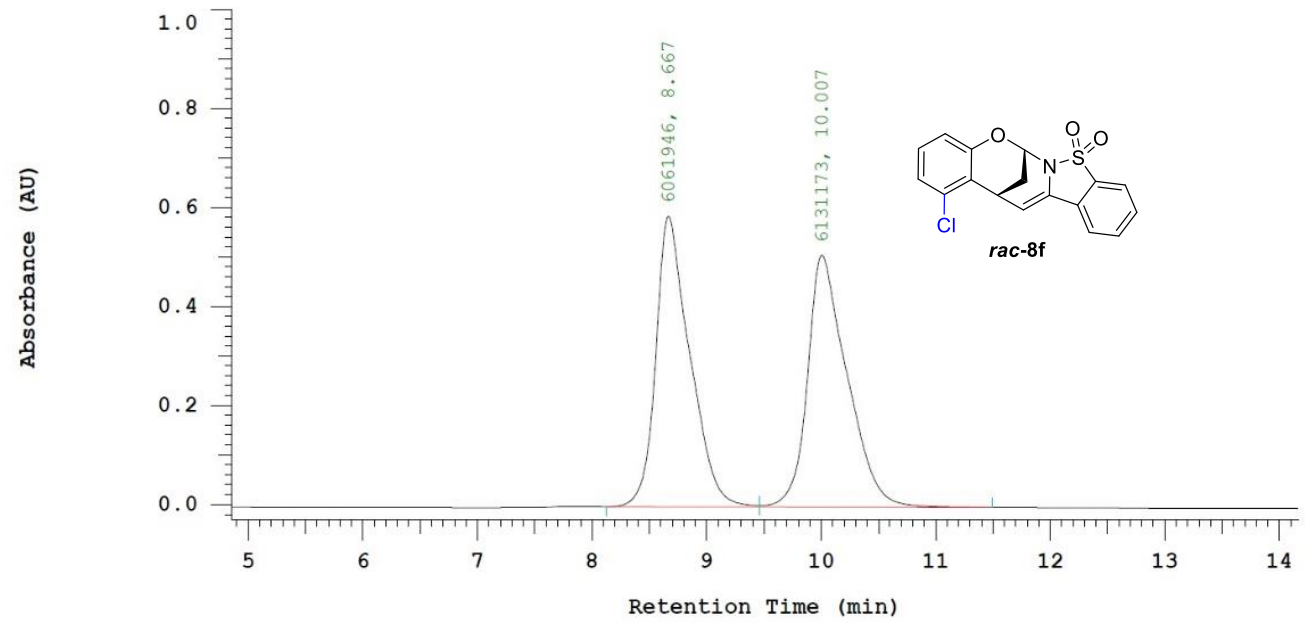

Chrom Type: Fixed WL Chromatogram, $210 \mathrm{~nm}$

Peak Quantitation: AREA

Calculation Method: AREA응

\begin{tabular}{crccc} 
No. & \multicolumn{1}{c}{ RT } & Area & Area & BC \\
\hline 1 & 8.667 & 6061946 & 49.716 & BV \\
2 & 10.007 & 6131173 & 50.284 & VB \\
\hline & & 12193119 & 100.000
\end{tabular}




\section{Chiral HPLC analysis of $\mathbf{8 f}$}

Chrom Type: Fixed WL Chromatogram, $210 \mathrm{~nm}$

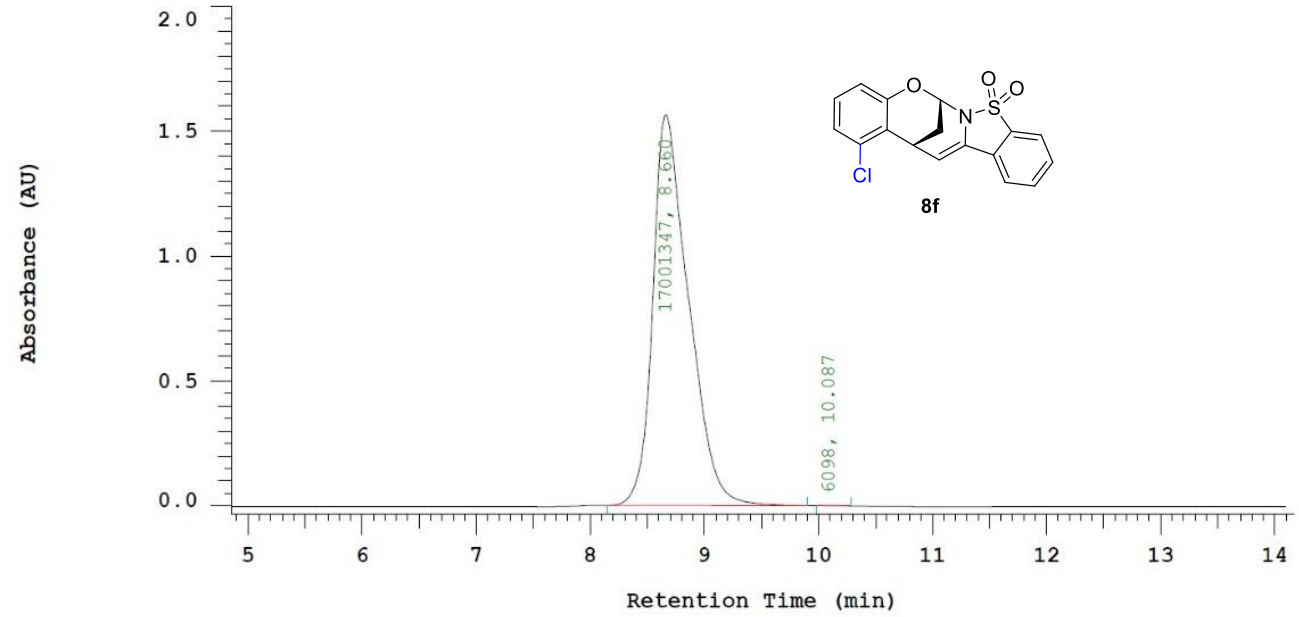

Chrom Type: Fixed WL Chromatogram, $210 \mathrm{~nm}$

Peak Quantitation: AREA

Calculation Method: AREA응

\begin{tabular}{rrrrr}
\multicolumn{1}{c}{ RT } & \multicolumn{1}{c}{ Area } & Area $\%$ & BC \\
\hline 1 & 8.660 & 17001347 & 99.964 & BB \\
2 & 10.087 & 6098 & 0.036 & BB \\
\hline & & 17007445 & 100.000
\end{tabular}

\section{${ }^{1} \mathrm{H}$ NMR of $8 \mathrm{~g}, \mathrm{CDCl}_{3}$}

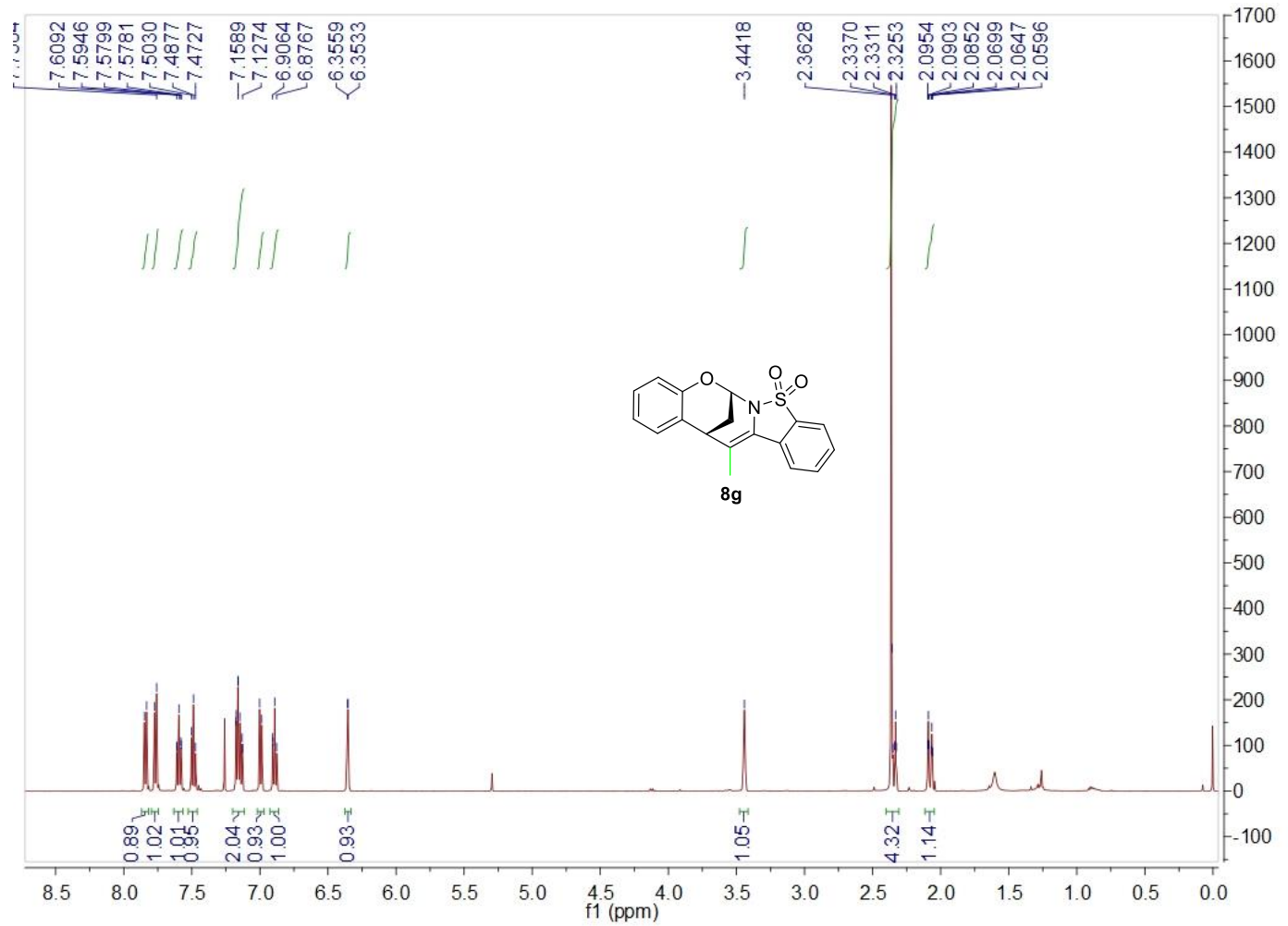


${ }^{13} \mathrm{CNMR}$ of $8 \mathrm{~g}, \mathrm{CDCl}_{3}$

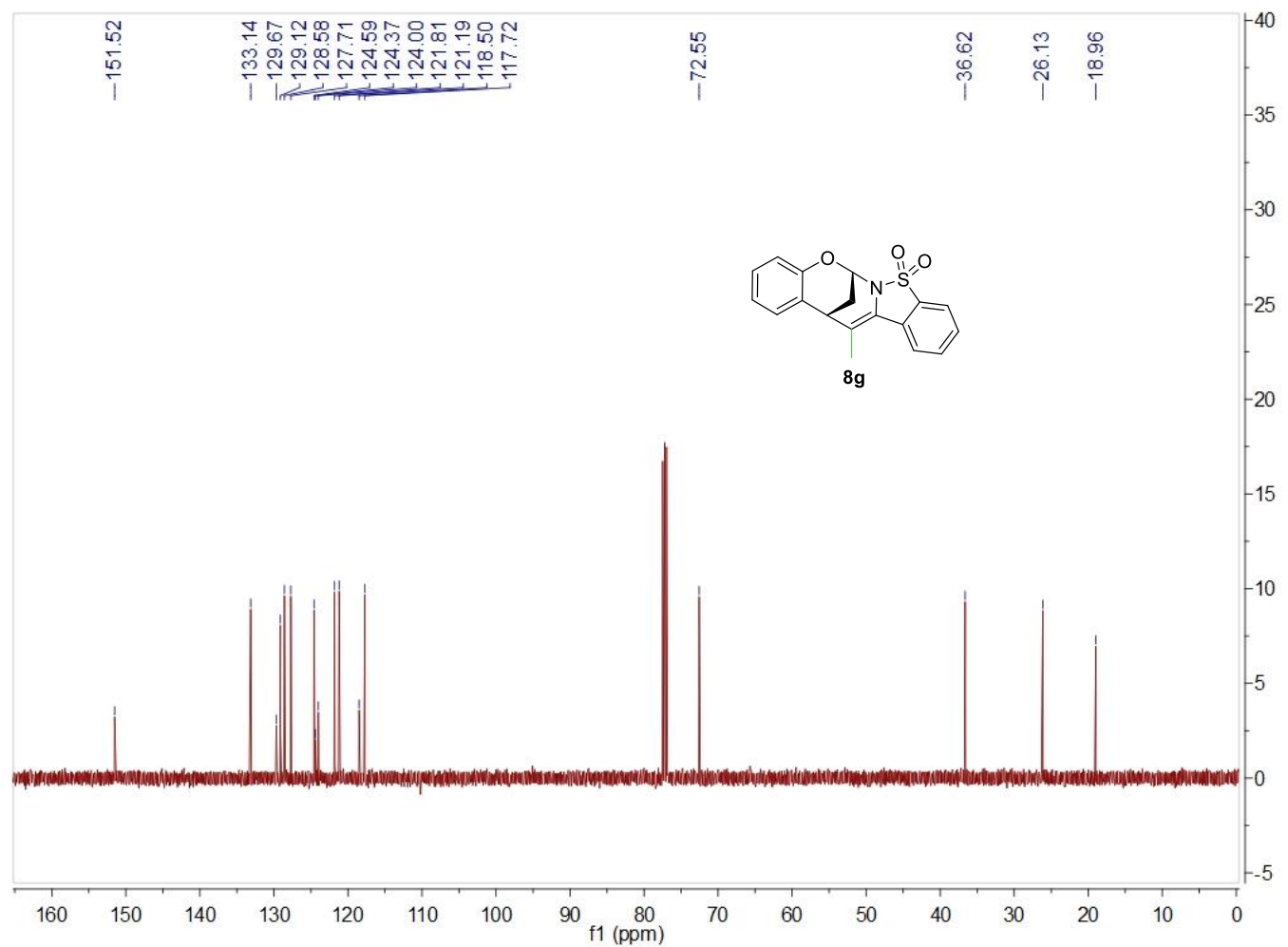

Chiral HPLC analysis of rac-8g

Chrom Type: Fixed WL Chromatogram, 216 nm

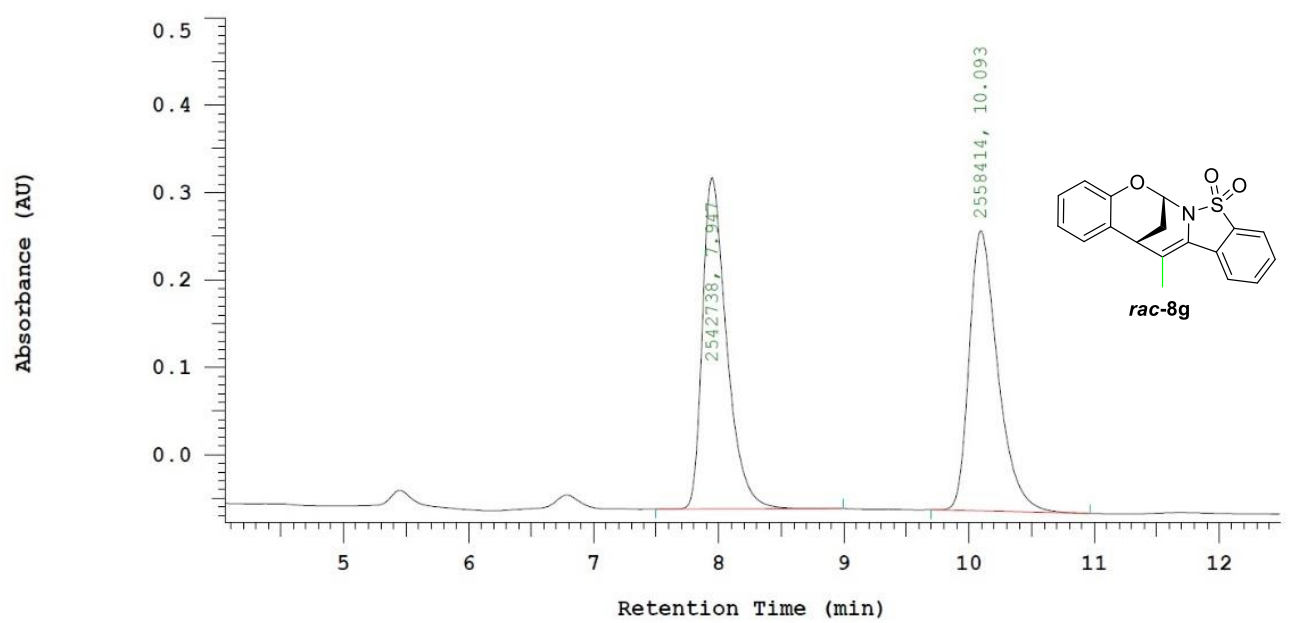

Chrom Type: Fixed WL Chromatogram, $216 \mathrm{~nm}$

Peak Quantitation: AREA

Calculation Method: AREA응

\begin{tabular}{rrrrr} 
No. & \multicolumn{1}{c}{ RT } & Area & Area & BC \\
\hline 1 & 7.947 & 2542738 & 49.846 & BB \\
2 & 10.093 & 2558414 & 50.154 & BB \\
\hline & & 5101152 & 100.000 & \\
\hline
\end{tabular}




\section{Chiral HPLC analysis of $\mathbf{8 g}$}

Chrom Type: Fixed WL Chromatogram, $216 \mathrm{~nm}$

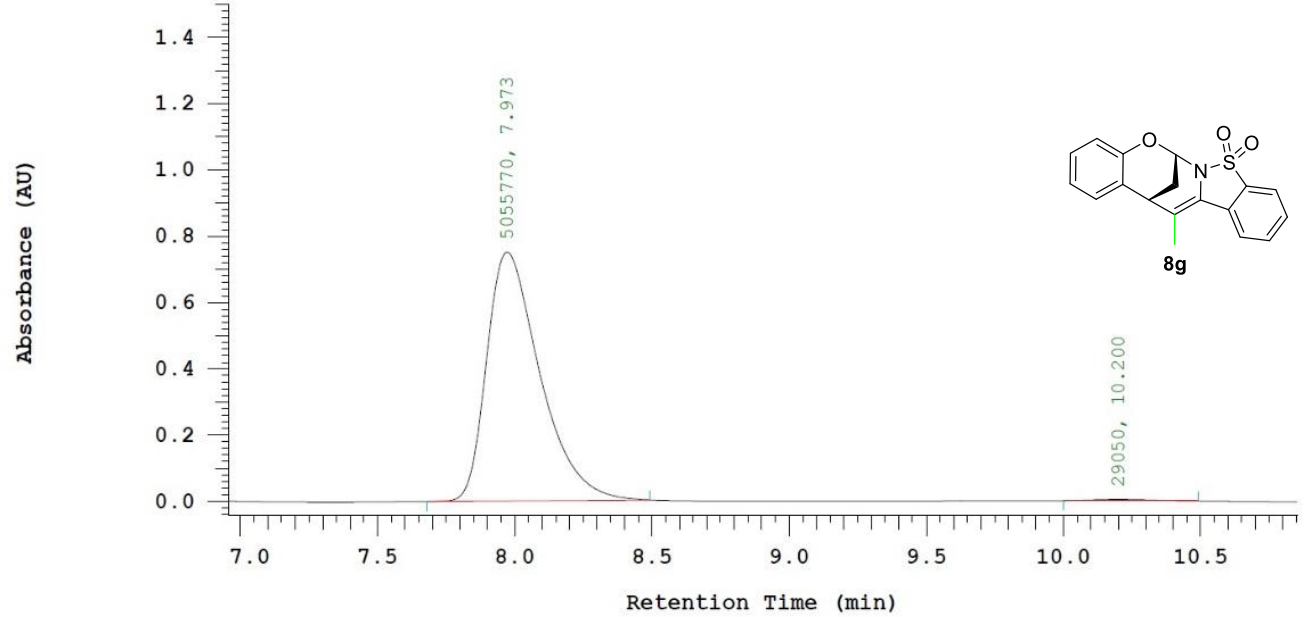

Chrom Type: Fixed WL Chromatogram, $216 \mathrm{~nm}$

Peak Quantitation: AREA

Calculation Method: AREA\%

\begin{tabular}{|c|c|c|c|c|}
\hline No. & $\mathrm{RT}$ & Area & Area 응 & $\mathrm{BC}$ \\
\hline 1 & 7.973 & 5055770 & 99.429 & $\mathrm{BB}$ \\
\hline 2 & 10.200 & 29050 & 0.571 & $\mathrm{BB}$ \\
\hline & & 5084820 & 100.000 & \\
\hline
\end{tabular}

${ }^{1} \mathrm{H} \mathrm{NMR}$ of $8 \mathrm{~h}, \mathrm{CDCl}_{3}$

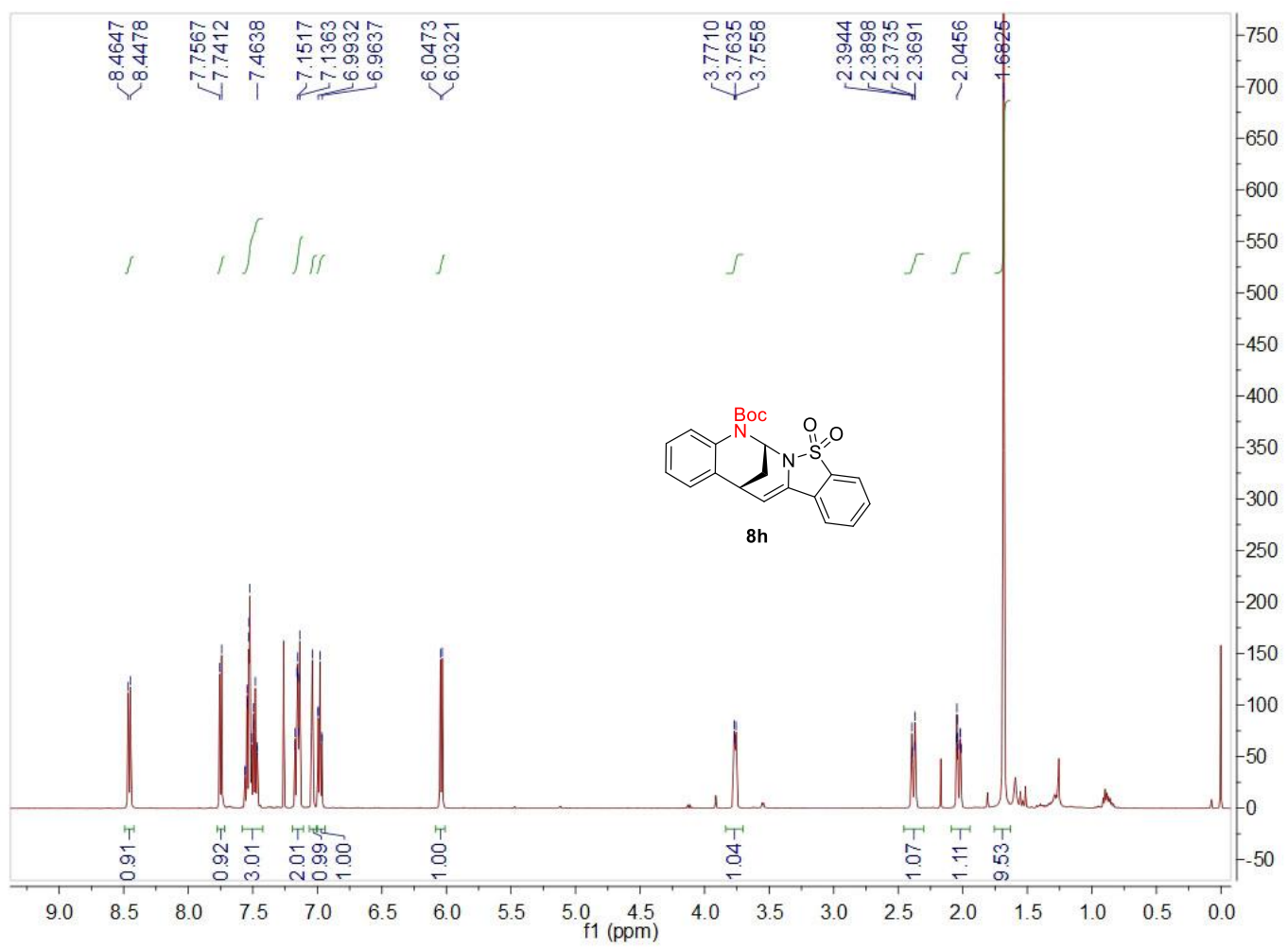


${ }^{13} \mathrm{C} \mathrm{NMR}$ of $8 \mathrm{~h}, \mathrm{CDCl}_{3}$

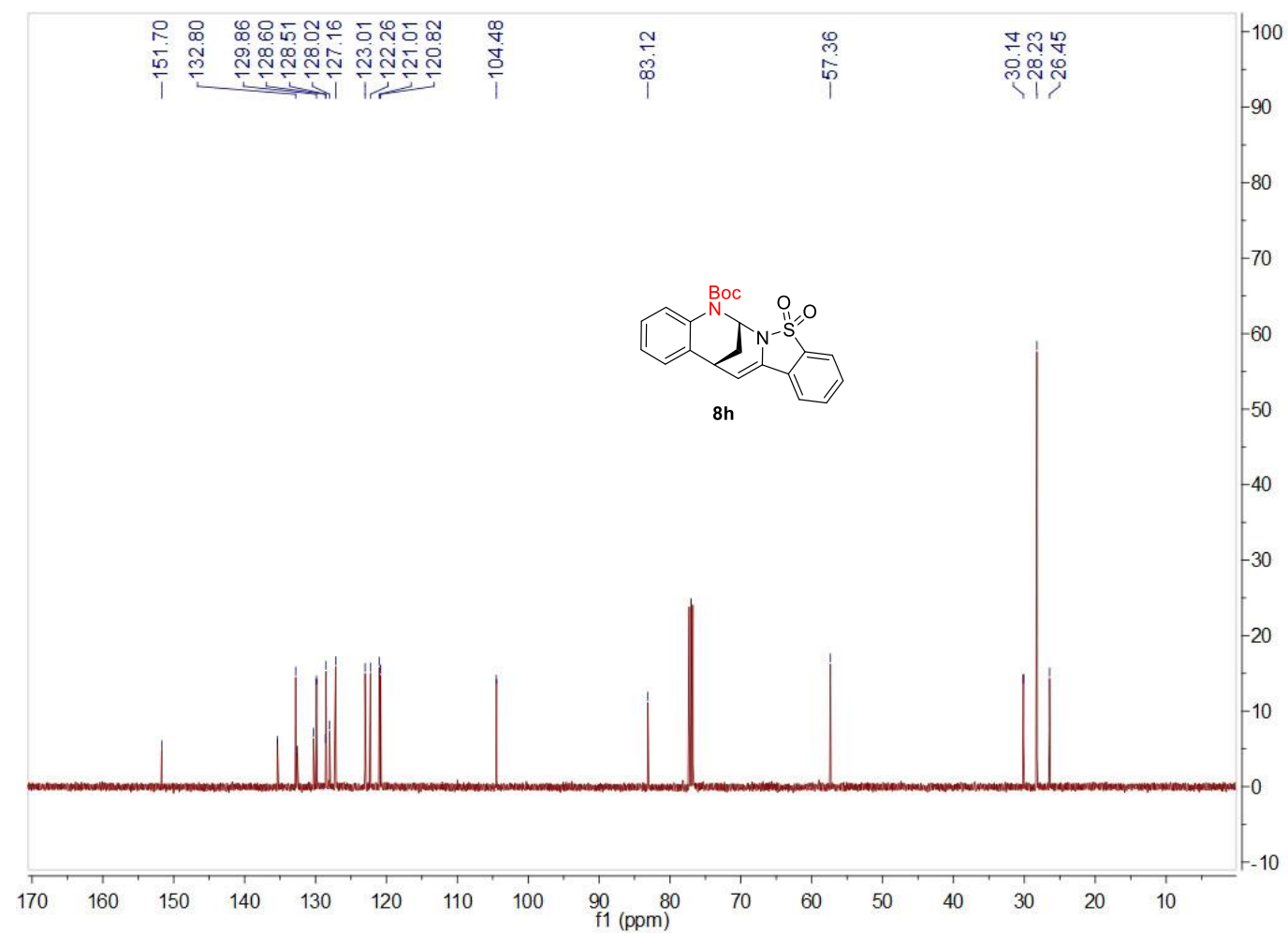

Chiral HPLC analysis of rac-8h

Chrom Type: Fixed WL Chromatogram, 225 nm

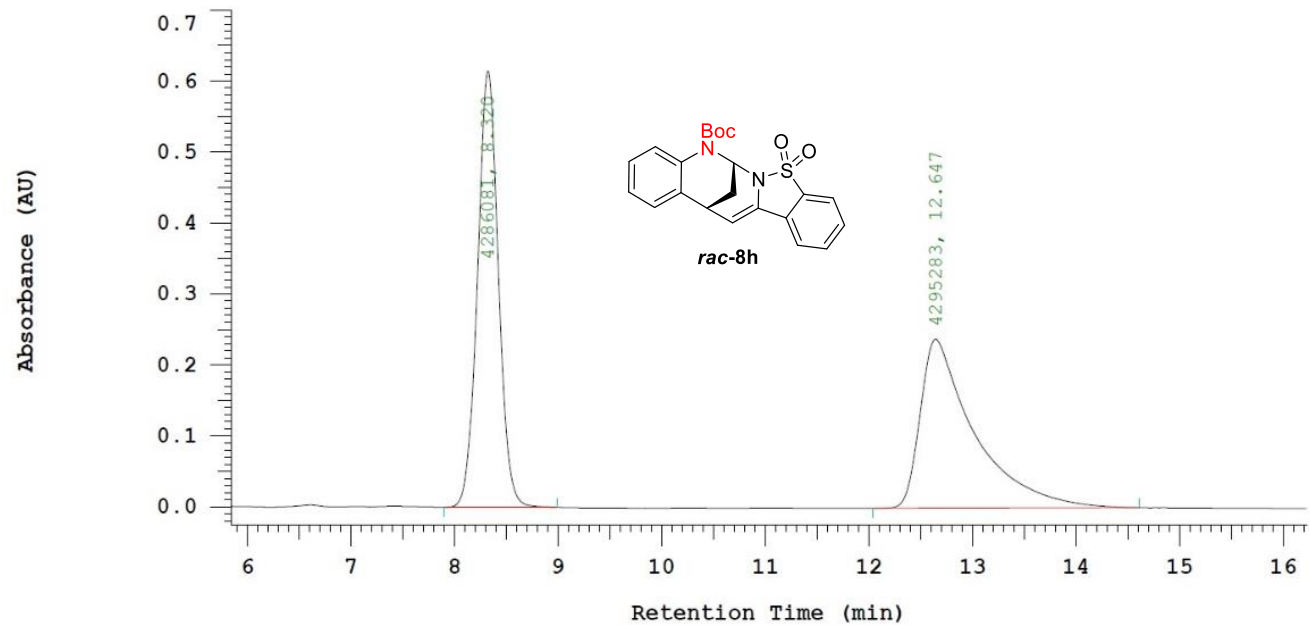

Chrom Type: Fixed WL Chromatogram, $225 \mathrm{~nm}$

Peak Quantitation: AREA

Calculation Method: AREA응

\begin{tabular}{|c|c|c|c|c|}
\hline No. & $\mathrm{RT}$ & Area & Area \% & $\mathrm{BC}$ \\
\hline 1 & 8.320 & 4286081 & 49.946 & BB \\
\hline 2 & 12.647 & 4295283 & 50.054 & $\mathrm{BB}$ \\
\hline & & 8581364 & 100.000 & \\
\hline
\end{tabular}




\section{Chiral HPLC analysis of $\mathbf{8 h}$}

Chrom Type: Fixed WL Chromatogram, 225 nm

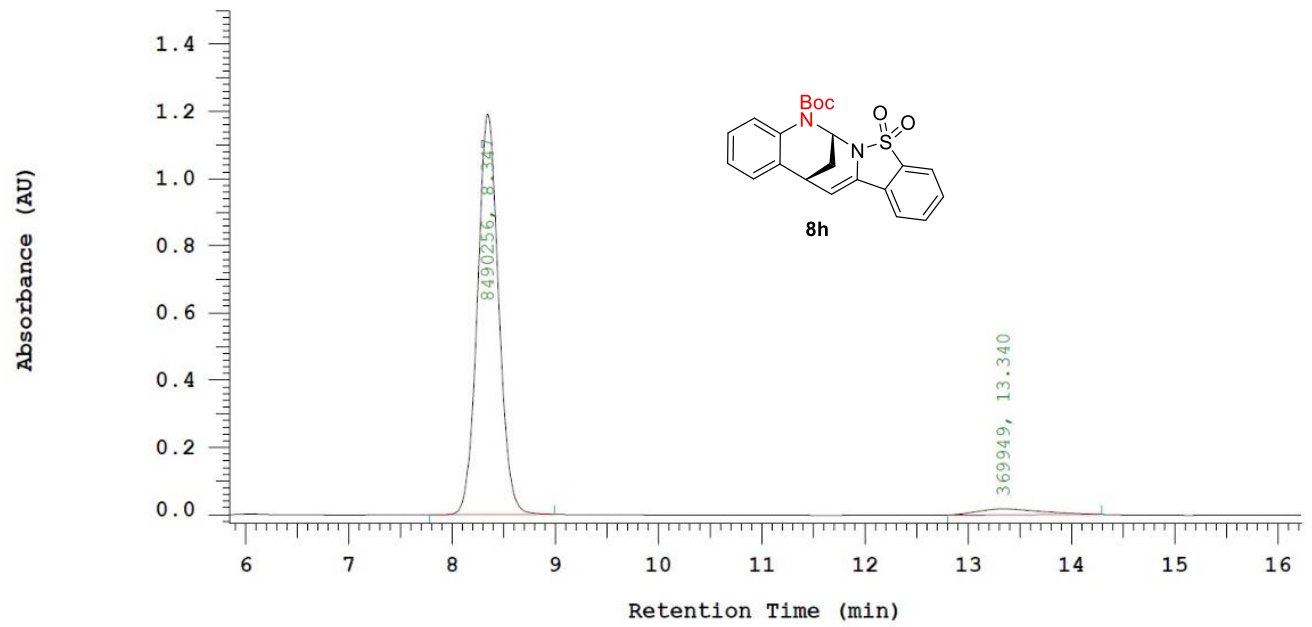

Chrom Type: Fixed WL Chromatogram, $225 \mathrm{~nm}$

Peak Quantitation: AREA

Calculation Method: AREA음

\begin{tabular}{|c|c|c|c|c|}
\hline No. & $\mathrm{RT}$ & Area & Area 응 & $\mathrm{BC}$ \\
\hline 1 & 8.347 & 8490256 & 95.825 & $\mathrm{BB}$ \\
\hline 2 & 13.340 & 369949 & 4.175 & $\mathrm{BB}$ \\
\hline & & 8860205 & 100.000 & \\
\hline
\end{tabular}

${ }^{1} \mathrm{H} N M R$ of $8 \mathbf{i}, \mathrm{CDCl}_{3}$

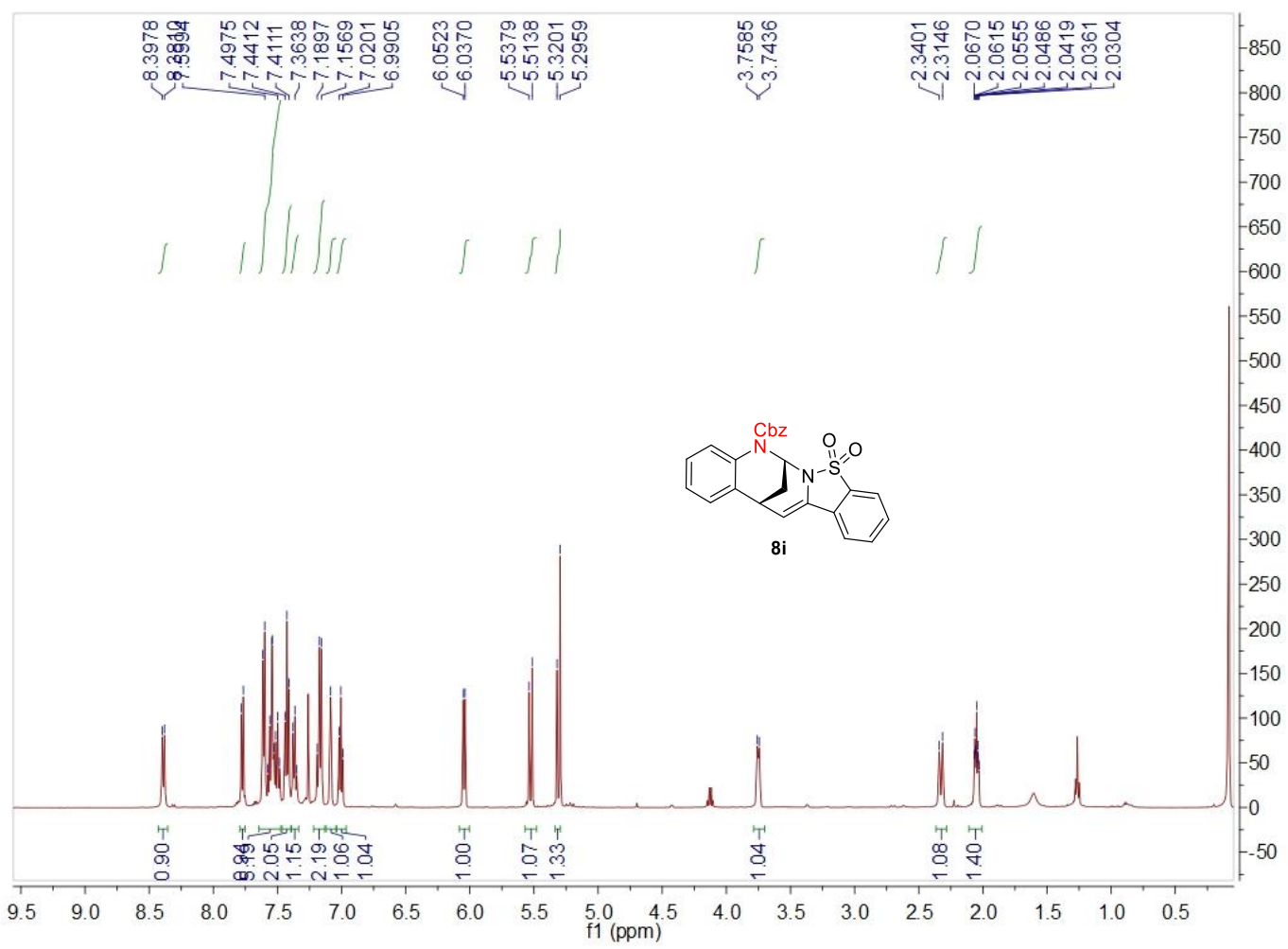


${ }^{13} \mathrm{CNMR}$ of $8 \mathrm{i}, \mathrm{CDCl}_{3}$

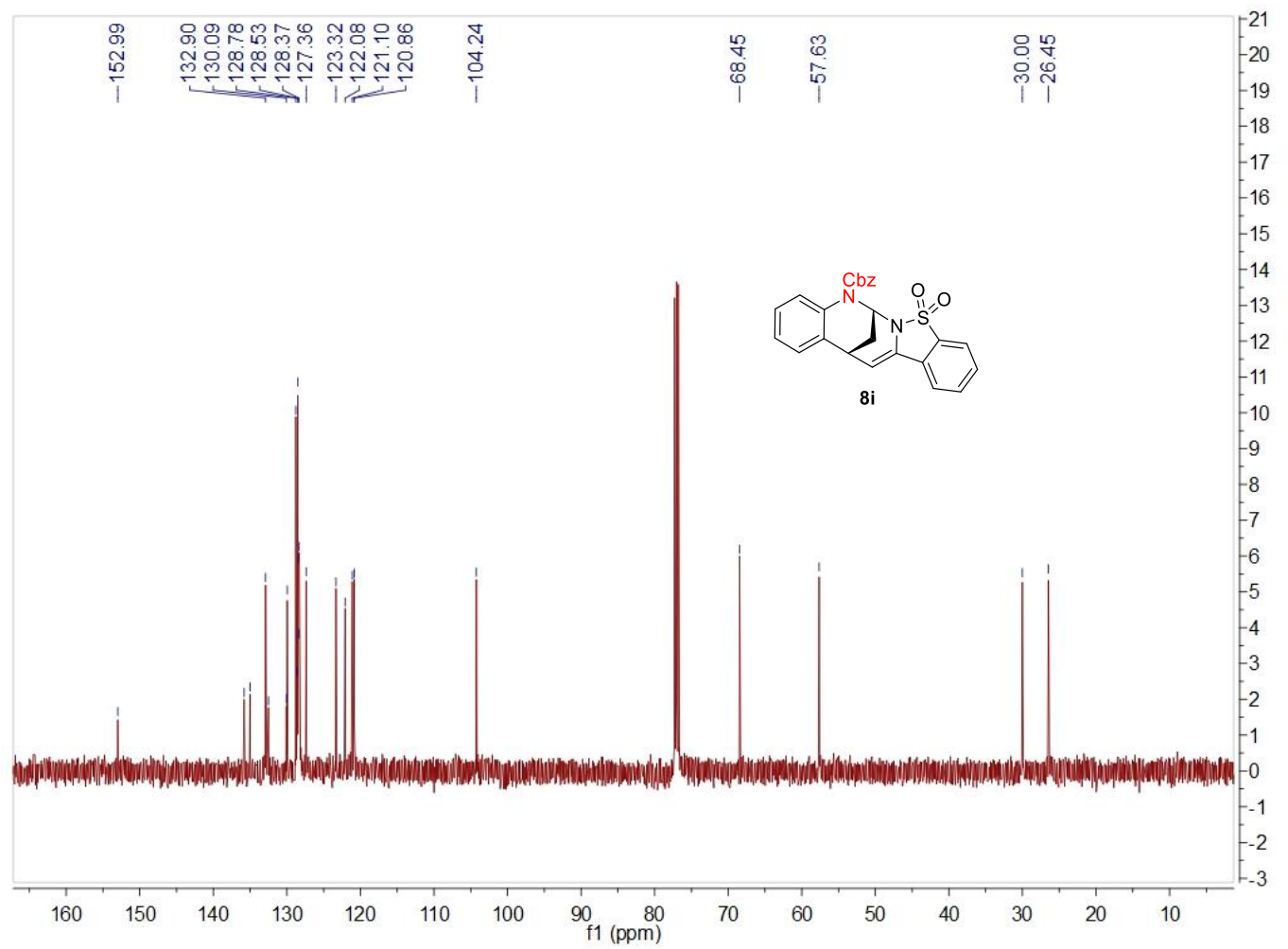

Chiral HPLC analysis of rac-8i

Chrom Type: Fixed WL Chromatogram, $225 \mathrm{~nm}$

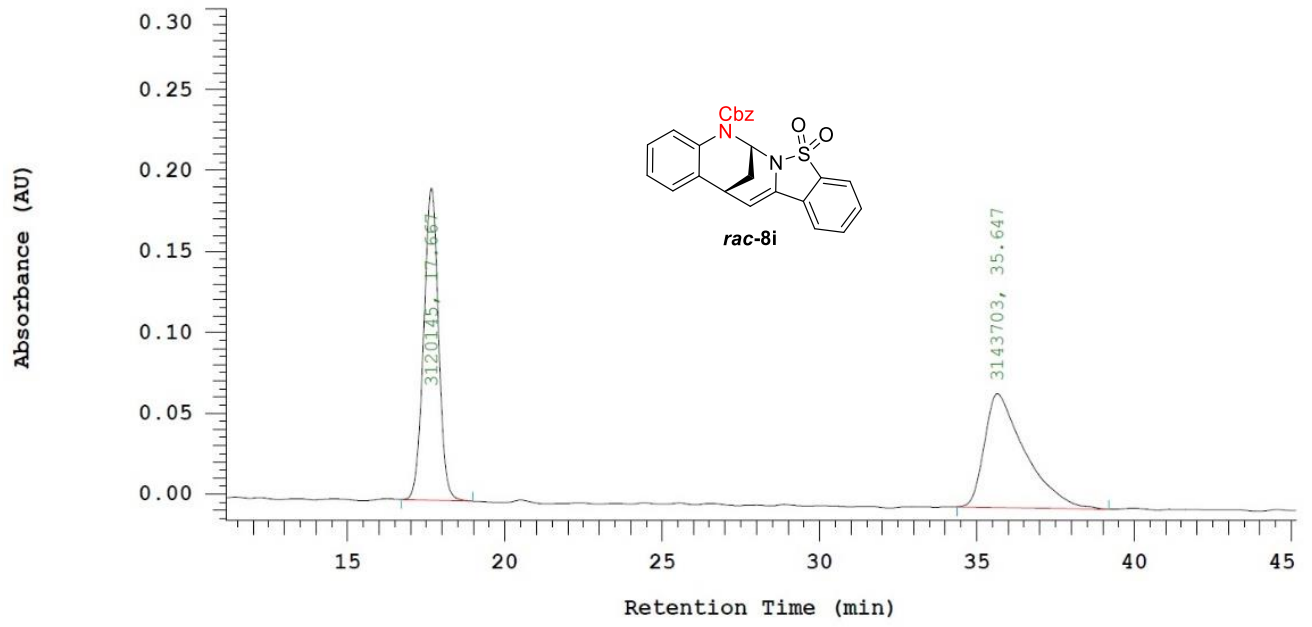

Chrom Type: Fixed WL Chromatogram, $225 \mathrm{~nm}$

Peak Quantitation: AREA

Calculation Method: AREA응

\begin{tabular}{ccccc} 
No. & RT & Area & Area & BC \\
\hline 1 & 17.667 & 3120145 & 49.812 & BB \\
2 & 35.647 & 3143703 & 50.188 & BB \\
\hline & & 6263848 & 100.000
\end{tabular}




\section{Chiral HPLC analysis of $\mathbf{8 i}$}

Chrom Type: Fixed WL Chromatogram, $225 \mathrm{~nm}$

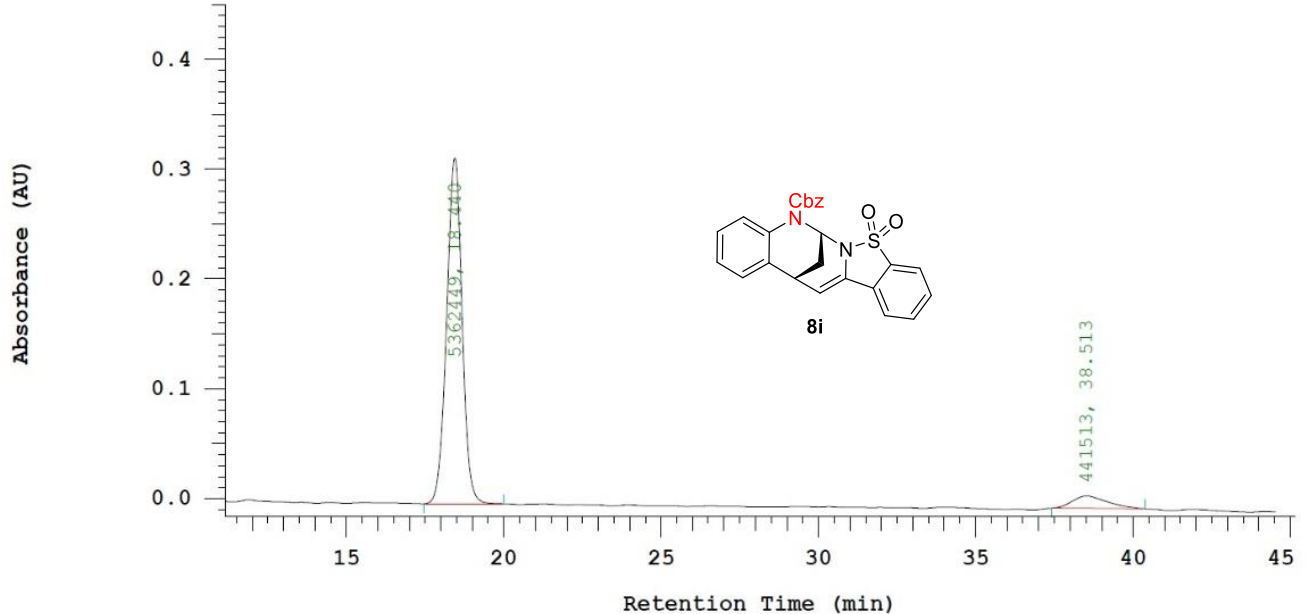

Chrom Type: Fixed WL Chromatogram, $225 \mathrm{~nm}$

Peak Quantitation: AREA

Calculation Method: AREA응

\begin{tabular}{|c|c|c|c|c|}
\hline ०. & $\mathrm{RT}$ & Area & Area 응 & $\mathrm{BC}$ \\
\hline 1 & 18.440 & 5362449 & 92.393 & BB \\
\hline 2 & 38.513 & 441513 & 7.607 & $\mathrm{BB}$ \\
\hline & & 5803962 & 100.000 & \\
\hline
\end{tabular}

${ }^{1} \mathrm{H} \mathrm{NMR}$ of $12 \mathrm{a}, \mathrm{CDCl}_{3}$

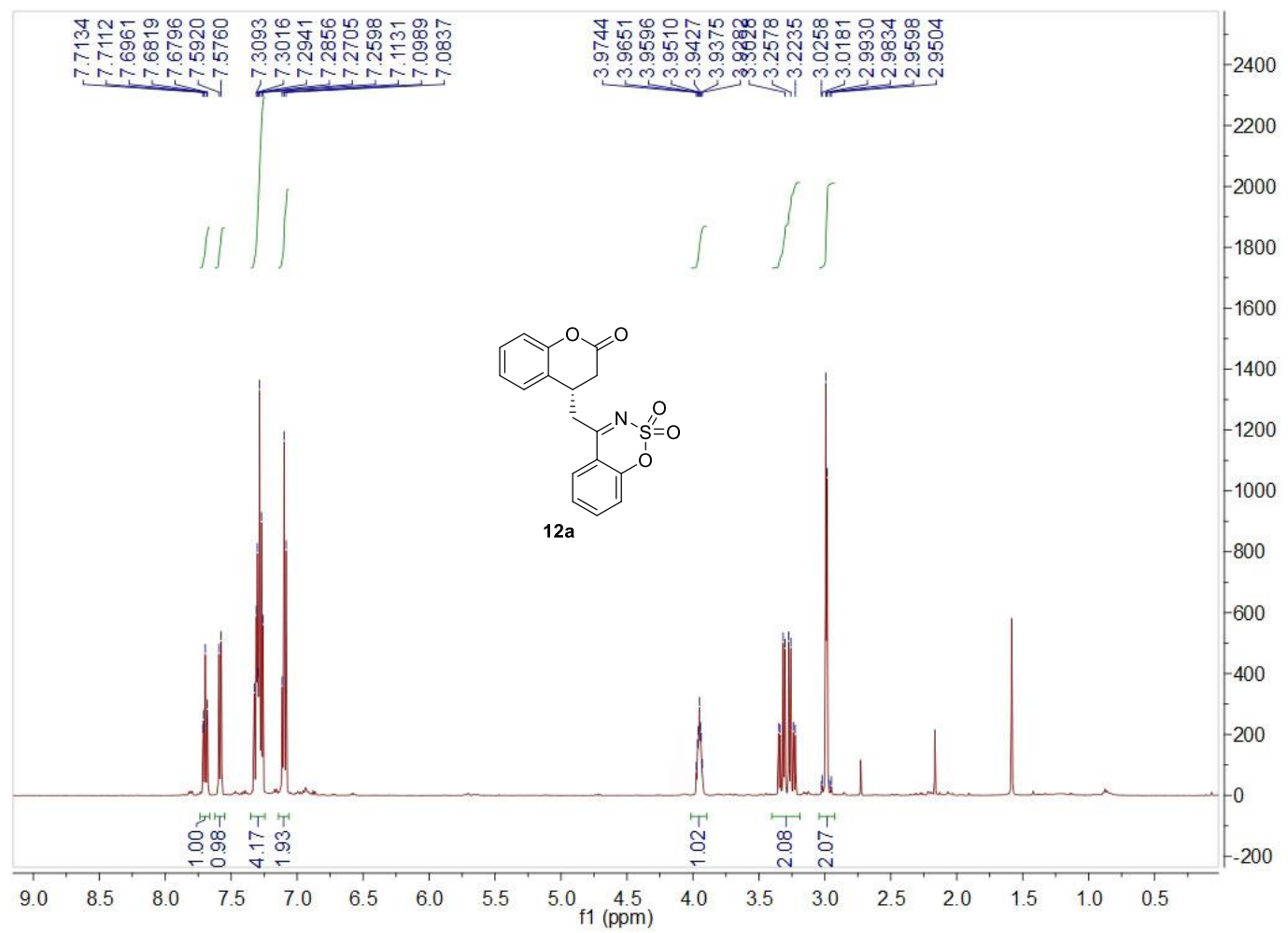


${ }^{13} \mathrm{C} \mathrm{NMR}$ of $12 \mathrm{a}, \mathrm{CDCl}_{3}$

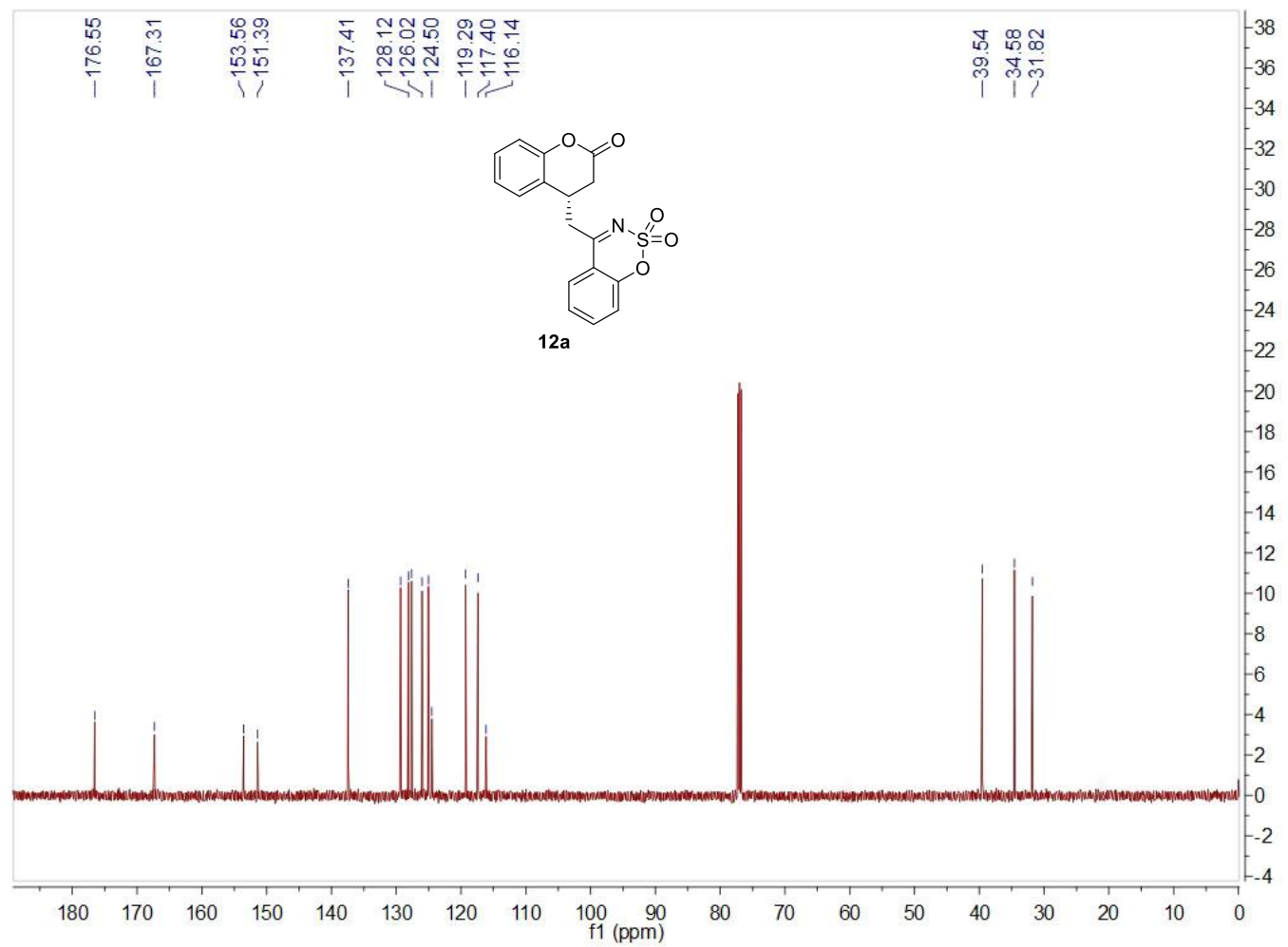

Chiral HPLC analysis of rac-12a

Chrom Type: Fixed WL Chromatogram, 270 nm

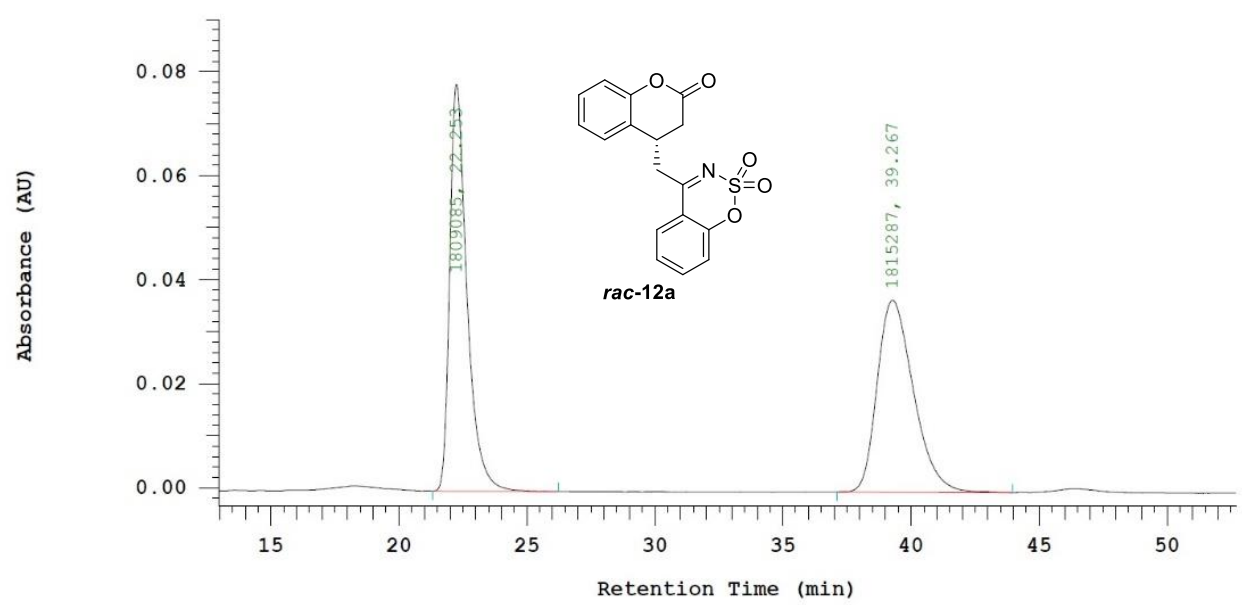

Chrom Type: Fixed WL Chromatogram, $270 \mathrm{~nm}$

Peak Quantitation: AREA

Calculation Method: AREA응

\begin{tabular}{ccccc} 
No. & RT & Area & Area & BC \\
\hline 1 & 22.253 & 1809085 & 49.914 & BB \\
2 & 39.267 & 1815287 & 50.086 & BB \\
\hline & & 3624372 & 100.000 &
\end{tabular}




\section{Chiral HPLC analysis of 12a}

Chrom Type: Fixed WL Chromatogram, 270 nm

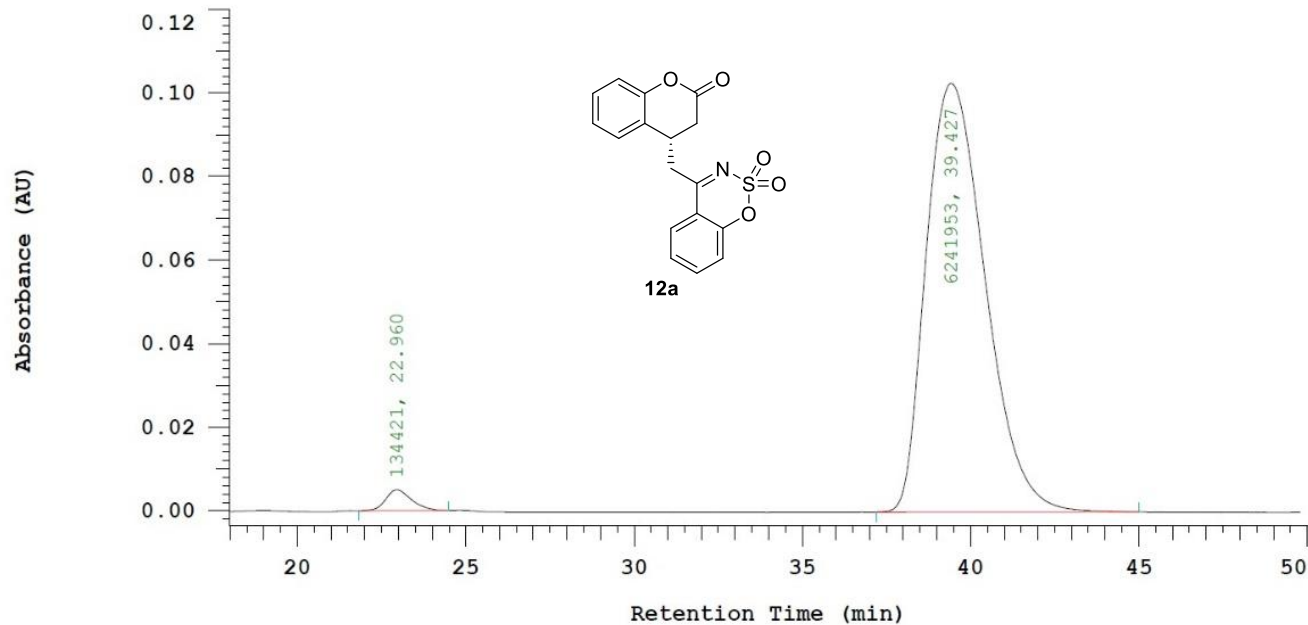

Chrom Type: Fixed WL Chromatogram, $270 \mathrm{~nm}$

Peak Quantitation: AREA

Calculation Method: AREA응

\begin{tabular}{|c|c|c|c|c|}
\hline To. & $\mathrm{RT}$ & Area & Area 응 & $\mathrm{BC}$ \\
\hline 1 & 22.960 & 134421 & 2.108 & BB \\
\hline 2 & 39.427 & 6241953 & 97.892 & $\mathrm{BB}$ \\
\hline & & 6376374 & 100.000 & \\
\hline
\end{tabular}

${ }^{1} \mathrm{H}$ NMR of $12 b, \mathrm{CDCl}_{3}$

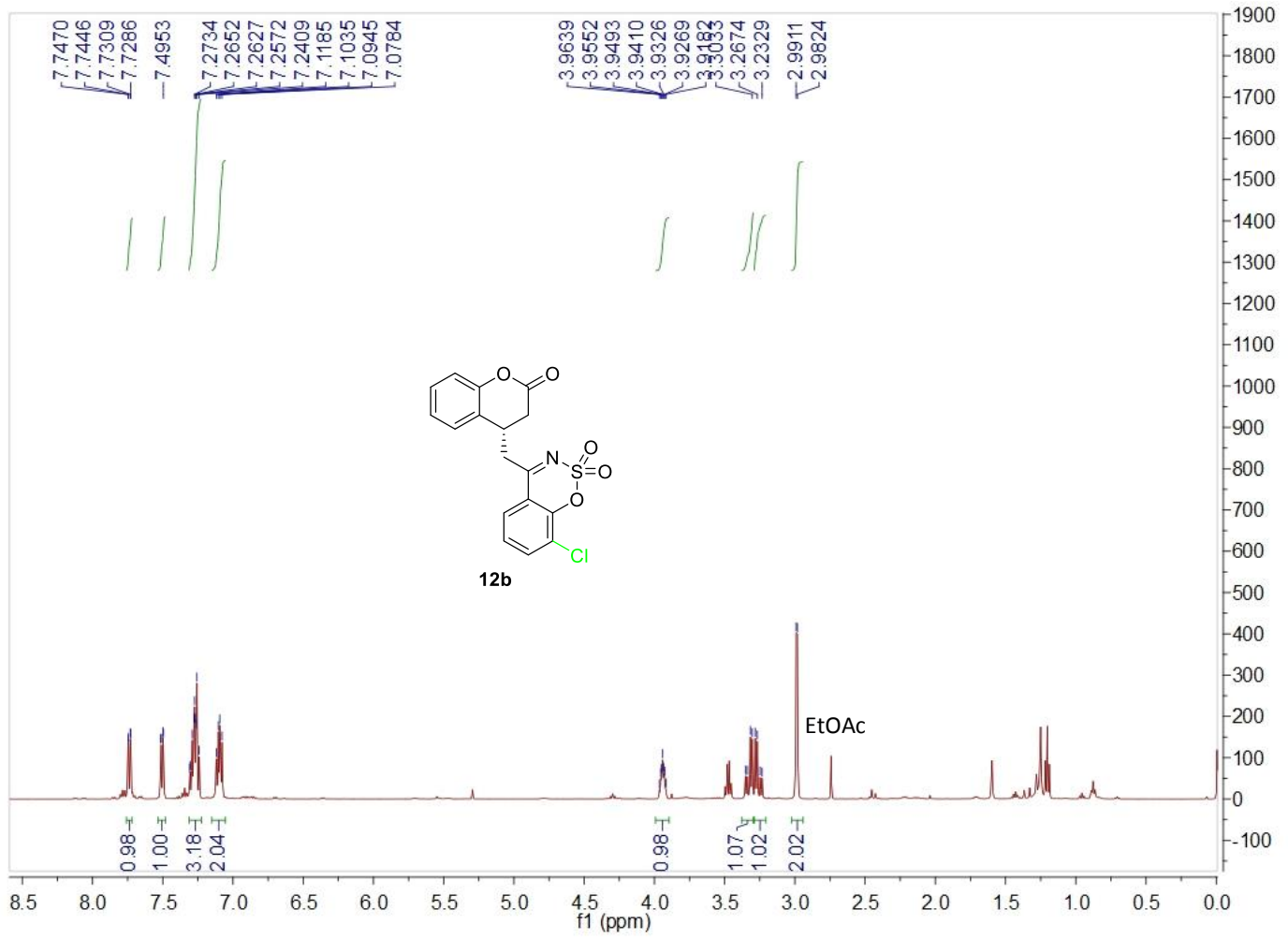


${ }^{13} \mathrm{C} \mathrm{NMR}$ of $\mathbf{1 2 b}, \mathrm{CDCl}_{3}$

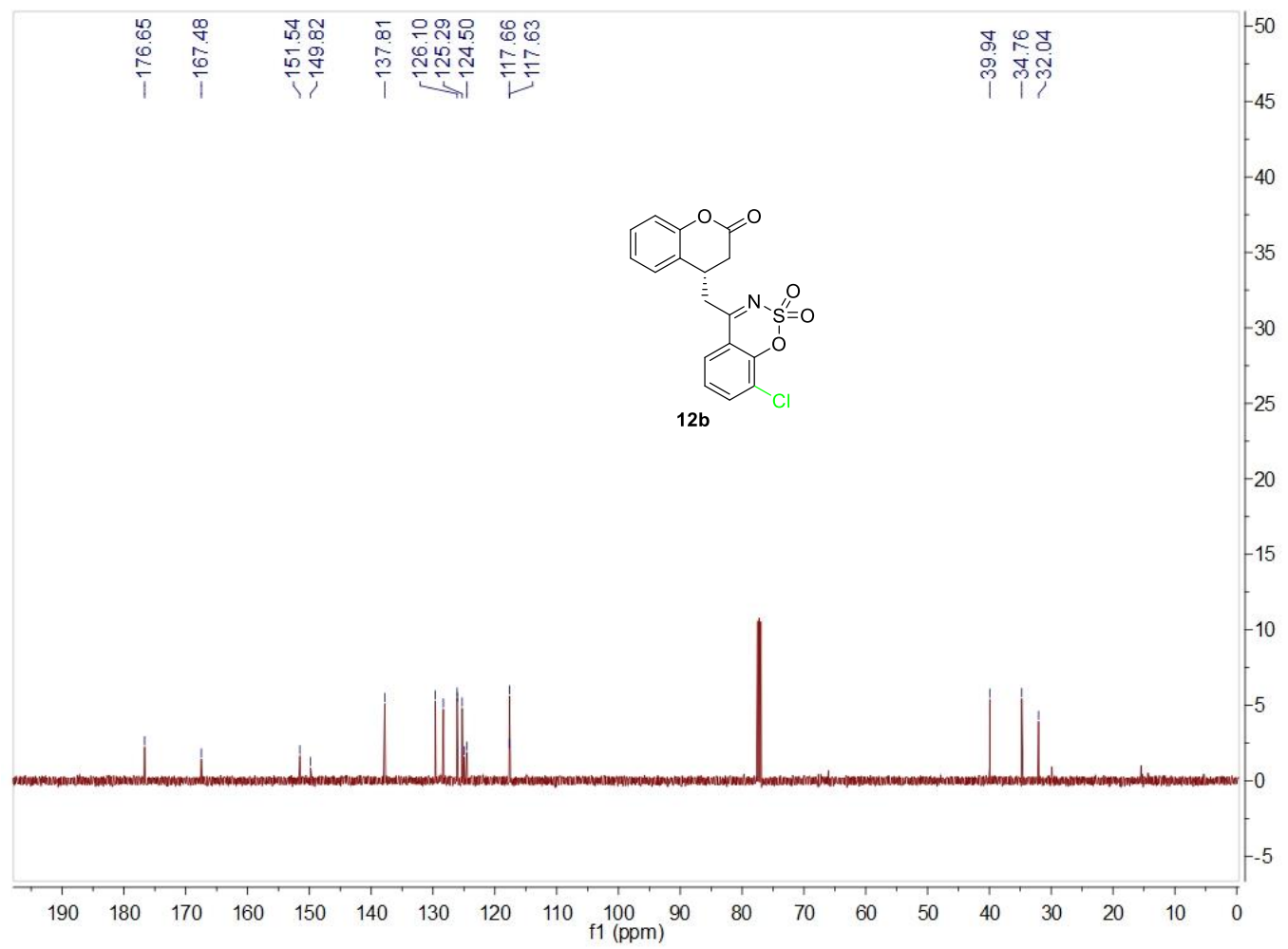

Chiral HPLC analysis of rac-12b

Chrom Type: Fixed WL Chromatogram, 260 nm

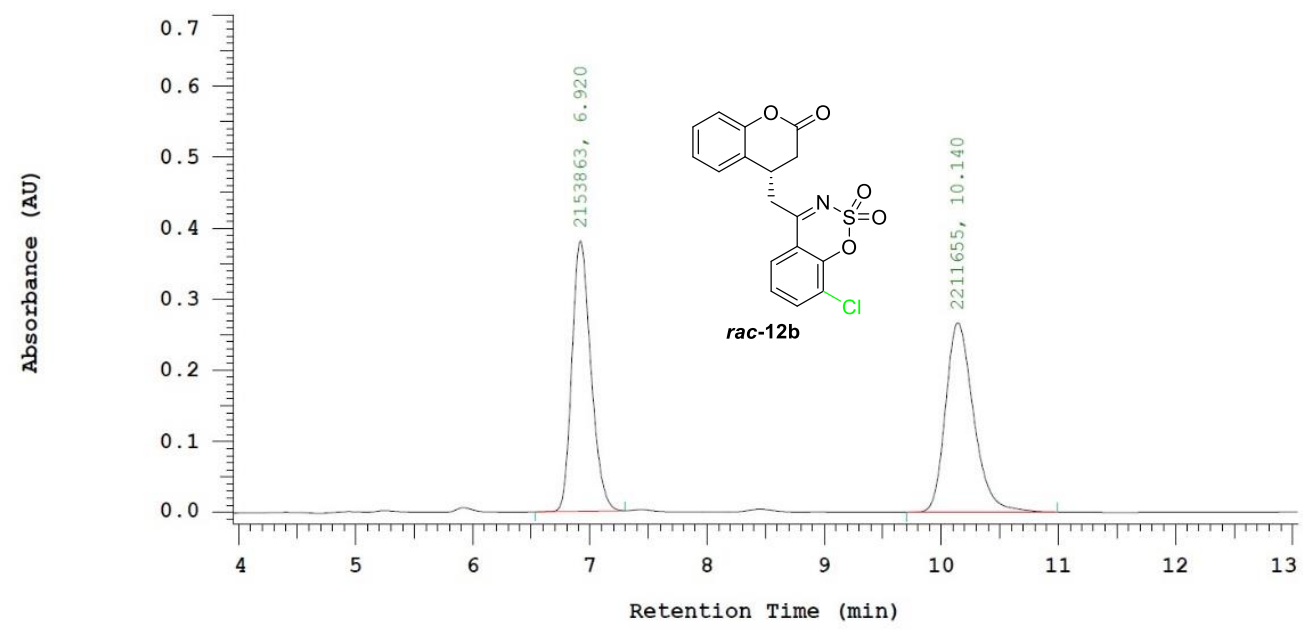

Chrom Type: Fixed WL Chromatogram, $260 \mathrm{~nm}$

Peak Quantitation: AREA

Calculation Method: AREA응

\begin{tabular}{rrrrr} 
No. & \multicolumn{1}{c}{ RT } & Area & Area & BC \\
\hline 1 & 6.920 & 2153863 & 49.338 & BB \\
2 & 10.140 & 2211655 & 50.662 & BB \\
\hline & & 4365518 & 100.000
\end{tabular}


Chiral HPLC analysis of $\mathbf{1 2 b}$

Chrom Type: Fixed WL Chromatogram, 260 nm

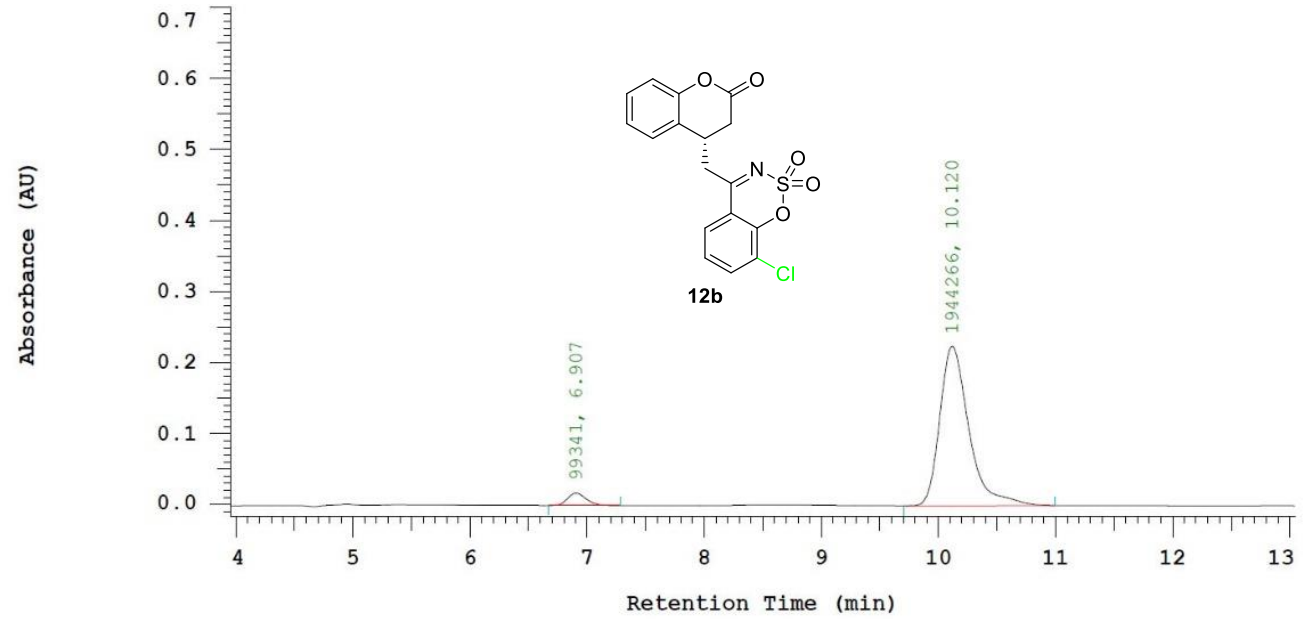

Chrom Type: Fixed WL Chromatogram, $260 \mathrm{~nm}$

Peak Quantitation: AREA

Calculation Method: AREA\%

\begin{tabular}{|c|c|c|c|c|}
\hline No. & $\mathrm{RT}$ & Area & Area 응 & $\mathrm{BC}$ \\
\hline 1 & 6.907 & 99341 & 4.861 & BB \\
\hline \multirow[t]{2}{*}{2} & 10.120 & 1944266 & 95.139 & BB \\
\hline & & 2043607 & 100.000 & \\
\hline
\end{tabular}

${ }^{1} \mathrm{H}$ NMR of $12 \mathrm{c}, \mathrm{CDCl}_{3}$

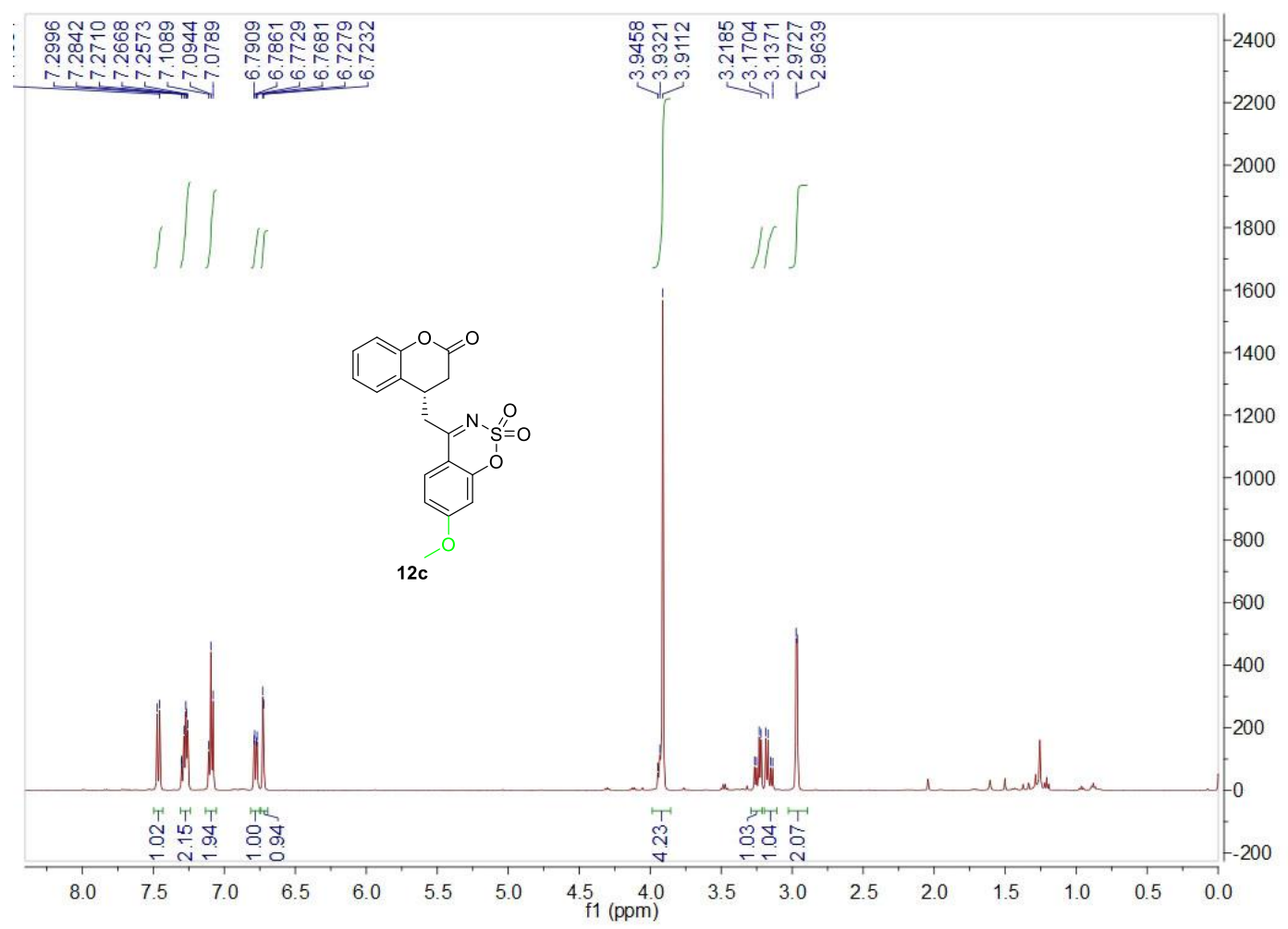


${ }^{13} \mathrm{C} \mathrm{NMR}$ of $12 \mathrm{c}, \mathrm{CDCl}_{3}$

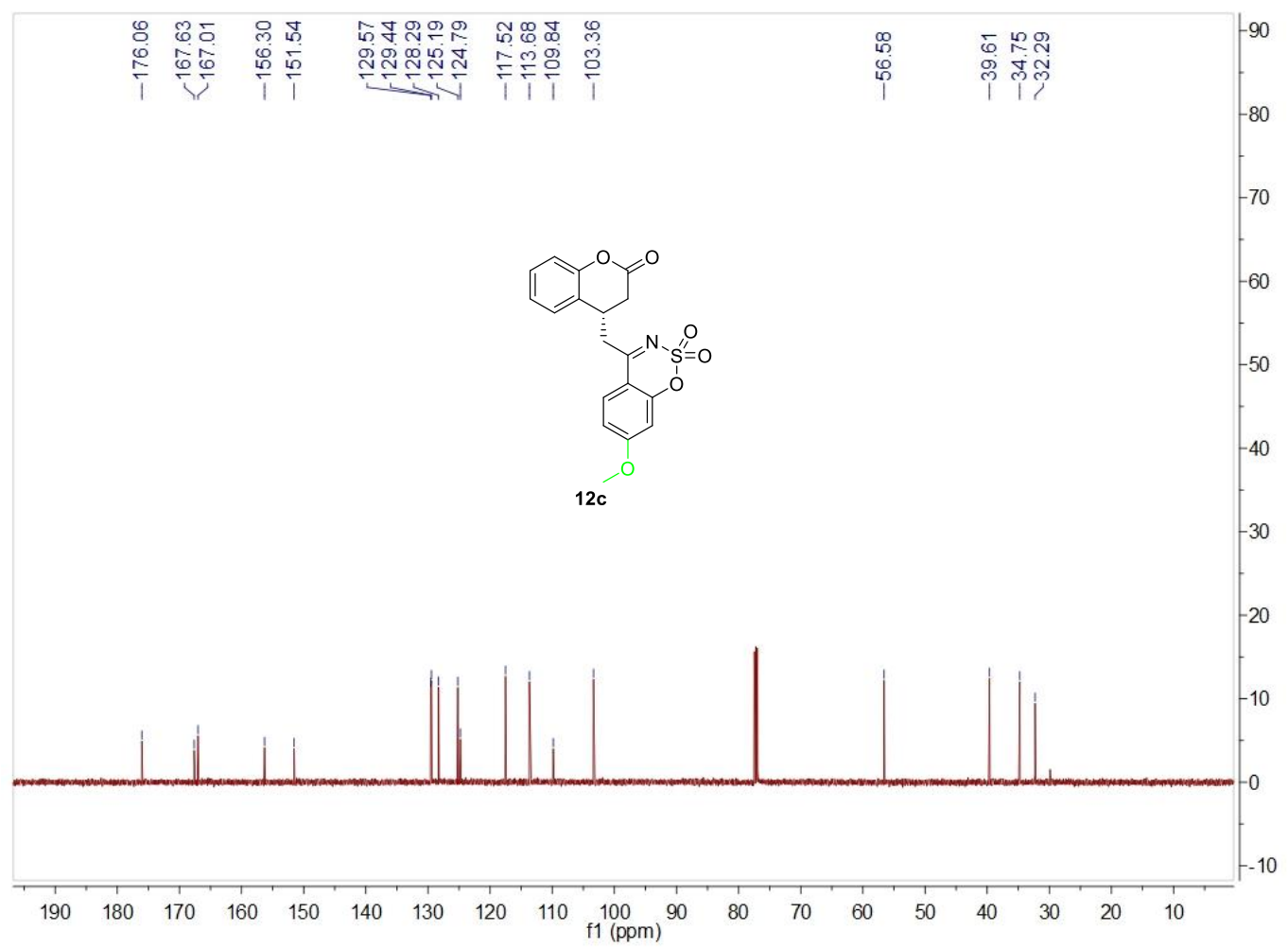

Chiral HPLC analysis of rac-12c

Chrom Type: Fixed WL Chromatogram, 299 nm

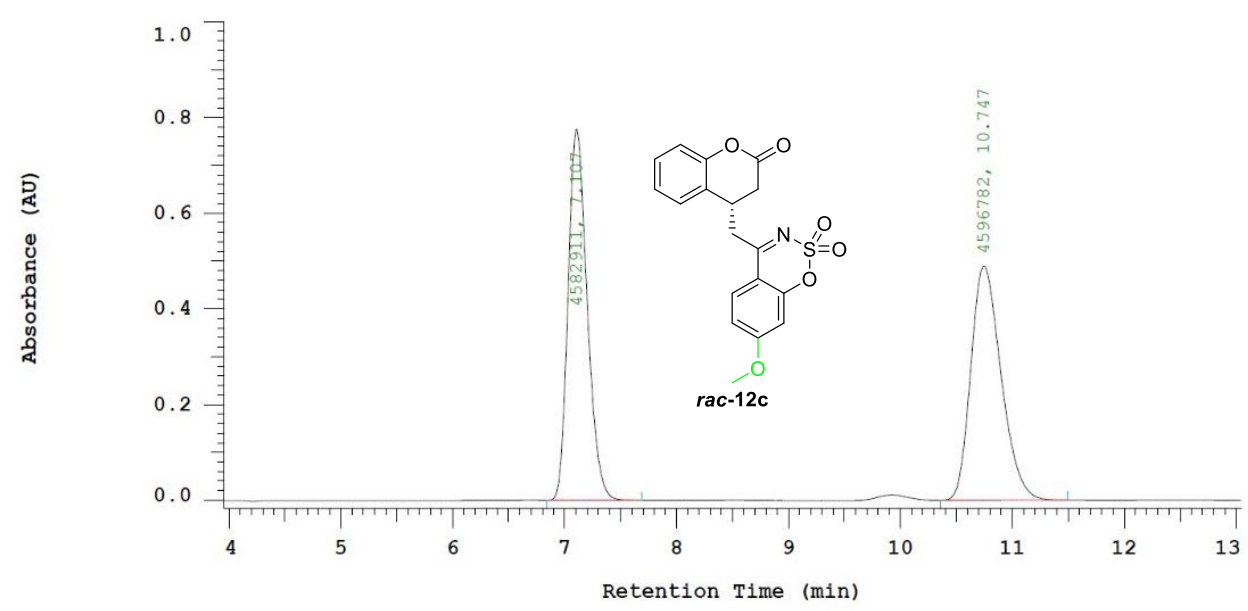

Chrom Type: Fixed WL Chromatogram, 299 nm

Peak Quantitation: AREA

Calculation Method: AREA\%

\begin{tabular}{rrrrr} 
No. & RT & Area & Area $\frac{\circ}{0}$ & BC \\
\hline 1 & 7.107 & 4582911 & 49.924 & BB \\
2 & 10.747 & 4596782 & 50.076 & BB \\
\hline & & 9179693 & 100.000 & \\
\hline
\end{tabular}




\section{Chiral HPLC analysis of 12c}

Chrom Type: Fixed WL Chromatogram, 299 nm

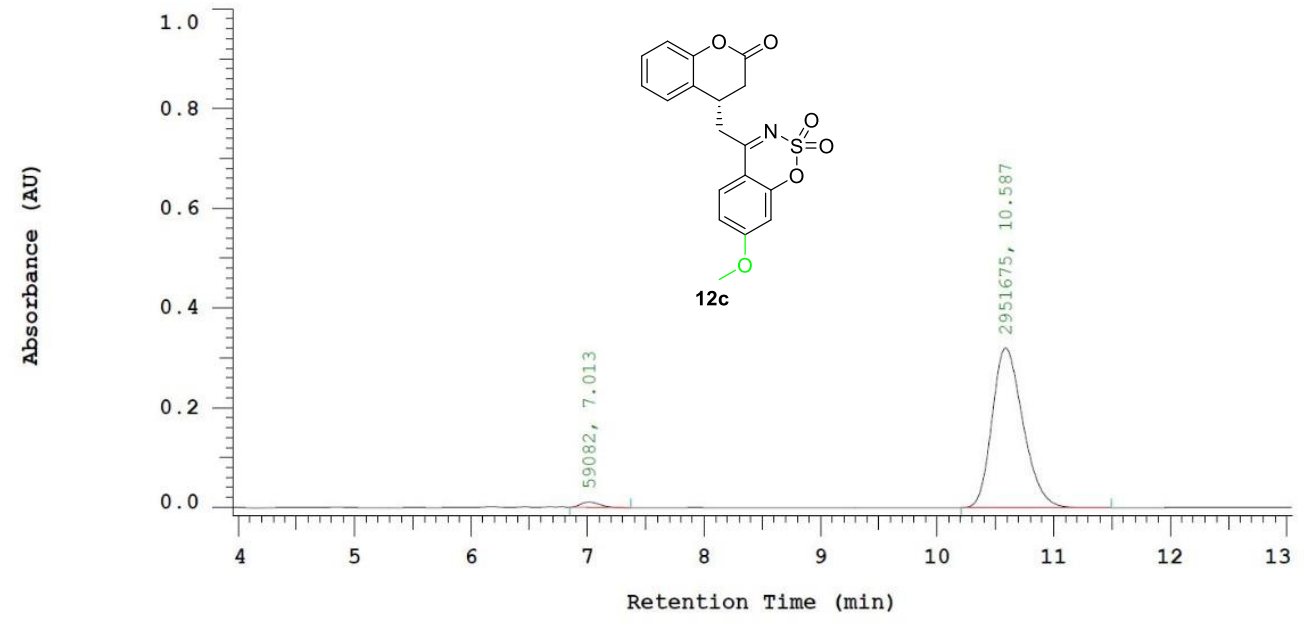

Chrom Type: Fixed WL Chromatogram, 299 nm

Peak Quantitation: AREA

Calculation Method: AREA\%

\begin{tabular}{|c|c|c|c|c|}
\hline No. & $\mathrm{RT}$ & Area & Area 응 & $\mathrm{BC}$ \\
\hline 1 & 7.013 & 59082 & 1.962 & BB \\
\hline 2 & 10.587 & 2951675 & 98.038 & BB \\
\hline & & 3010757 & 100.000 & \\
\hline
\end{tabular}

${ }^{1} \mathrm{H} \mathrm{NMR}$ of $12 \mathrm{~d}, \mathrm{CDCl}_{3}$

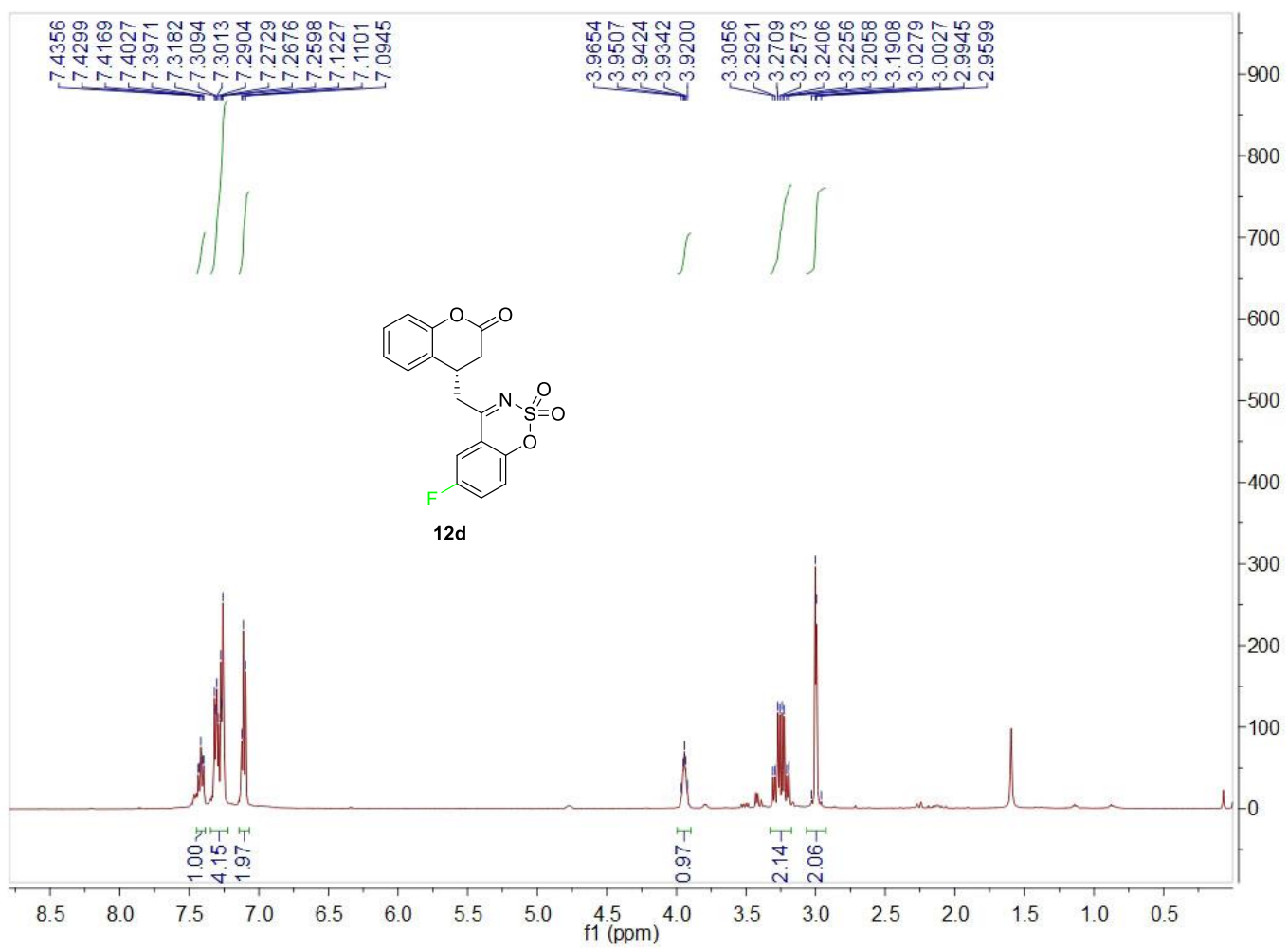


${ }^{13} \mathrm{CNMR}$ of $12 \mathrm{~d}, \mathrm{CDCl}_{3}$

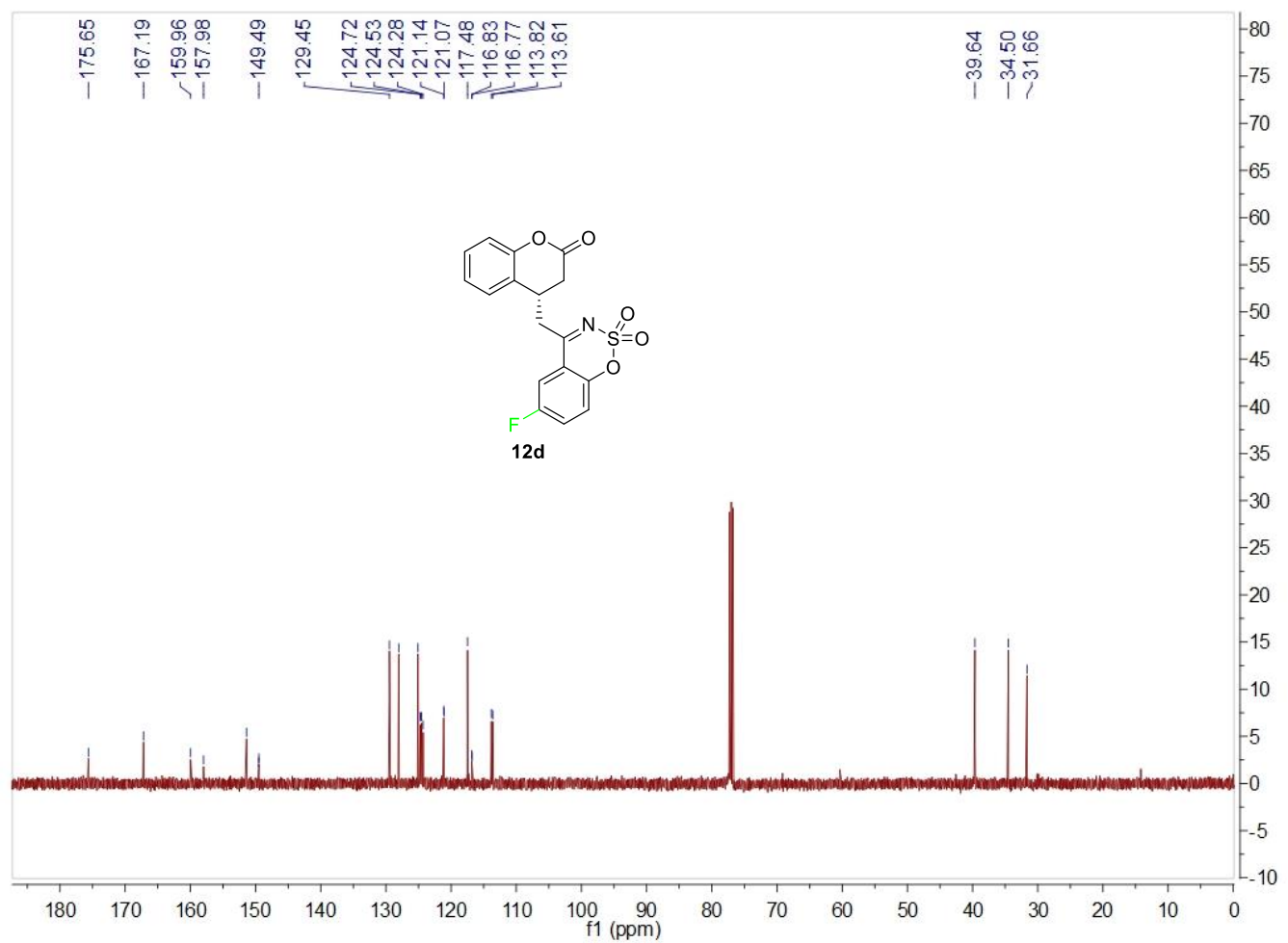

Chiral HPLC analysis of rac-12d

Chrom Type: Fixed WL Chromatogram, 260 nm

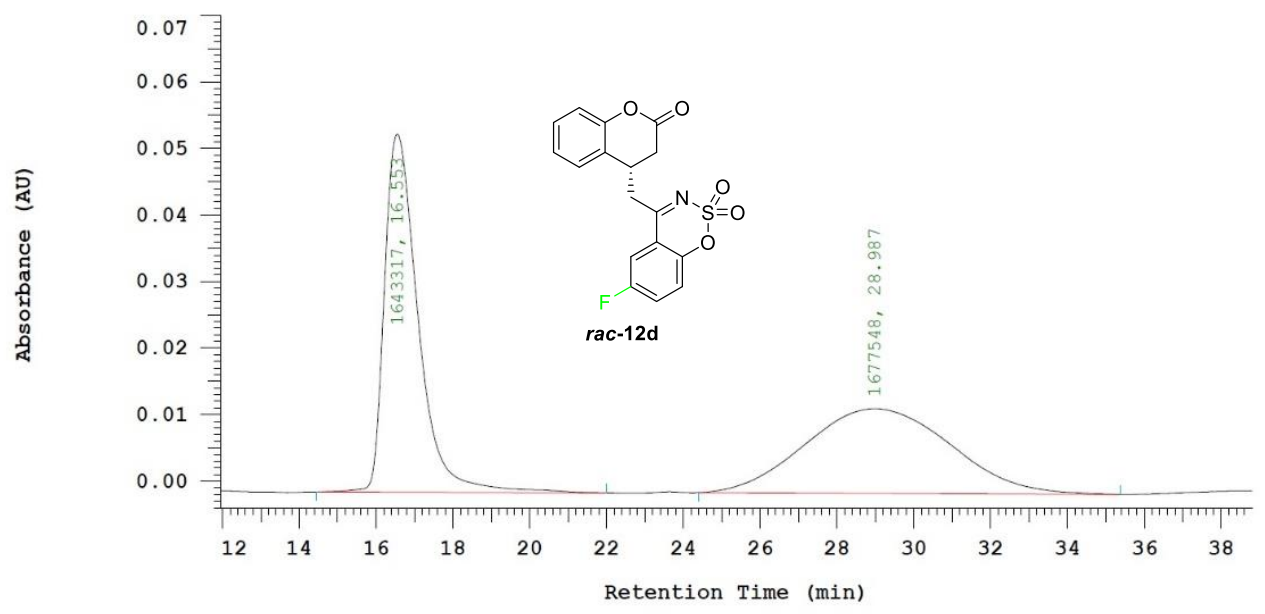

Chrom Type: Fixed WL Chromatogram, 260 nm Peak Quantitation: AREA

Calculation Method: AREA응

\begin{tabular}{ccccc} 
No. & RT & Area & Area $\%$ & BC \\
\hline 1 & 16.553 & 1643317 & 49.485 & BB \\
2 & 28.987 & 1677548 & 50.515 & BB \\
\hline & & 3320865 & 100.000 & \\
\hline
\end{tabular}




\section{Chiral HPLC analysis of 12d}

Chrom Type: Fixed WL Chromatogram, 260 nm

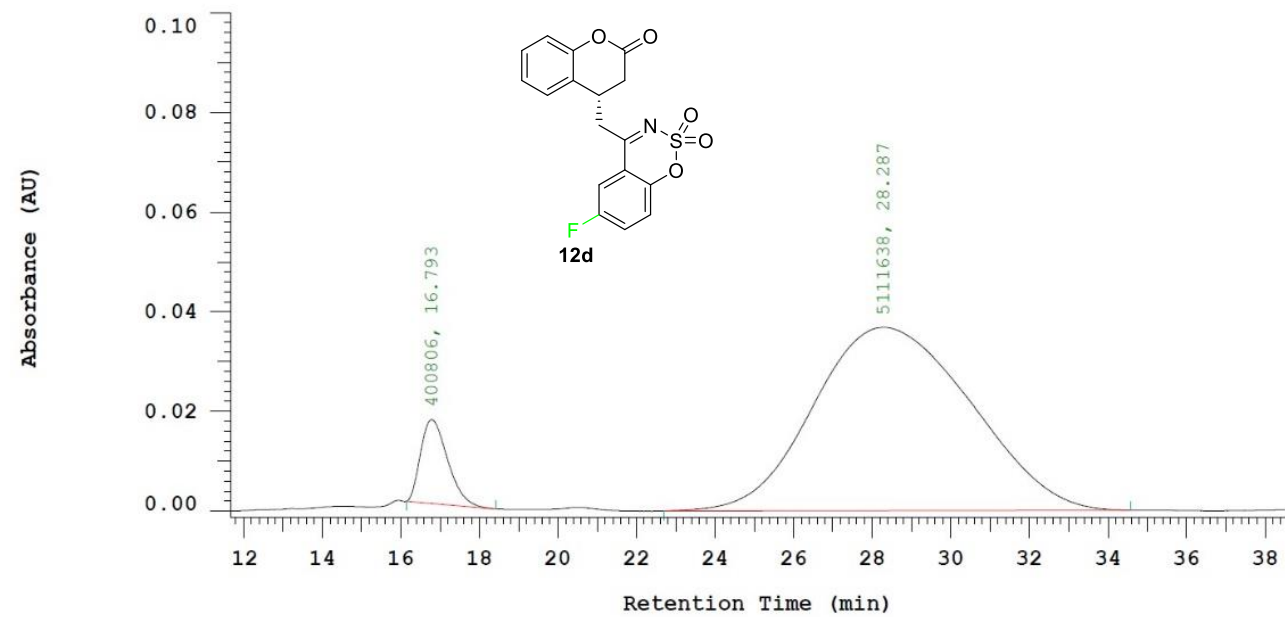

Chrom Type: Fixed WL Chromatogram, 260 nm

Peak Quantitation: AREA

Calculation Method: AREA응

\begin{tabular}{crrrr} 
No. & RT & \multicolumn{1}{c}{ Area } & Area $\%$ & BC \\
\hline 1 & 16.793 & 400806 & 7.271 & BB \\
2 & 28.287 & 5111638 & 92.729 & BB \\
\hline & & 5512444 & 100.000
\end{tabular}

${ }^{1} \mathrm{H}$ NMR of $12 \mathrm{e}, \mathrm{CDCl}_{3}$

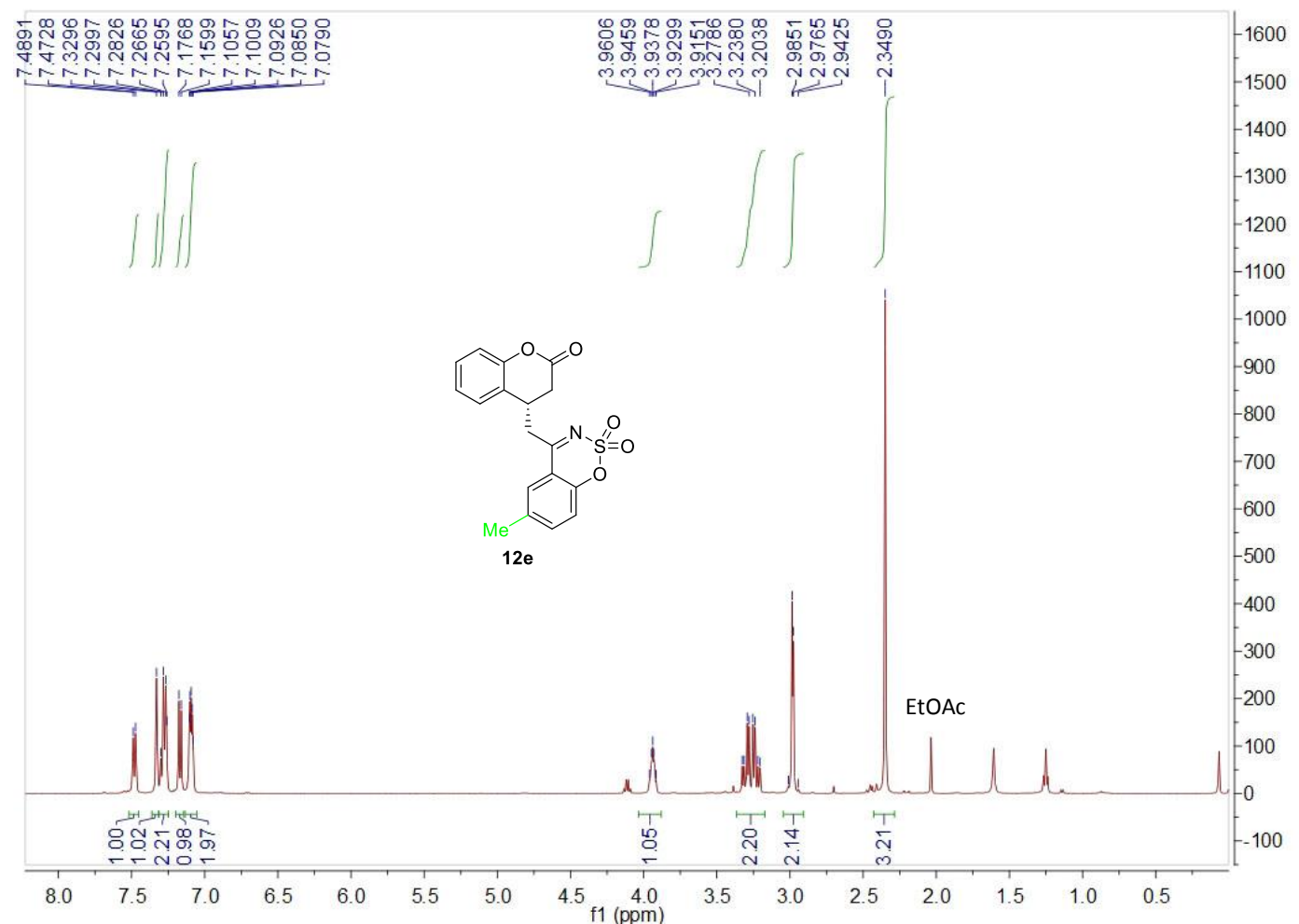


${ }^{13} \mathrm{C} \mathrm{NMR}$ of $12 \mathrm{e}, \mathrm{CDCl}_{3}$

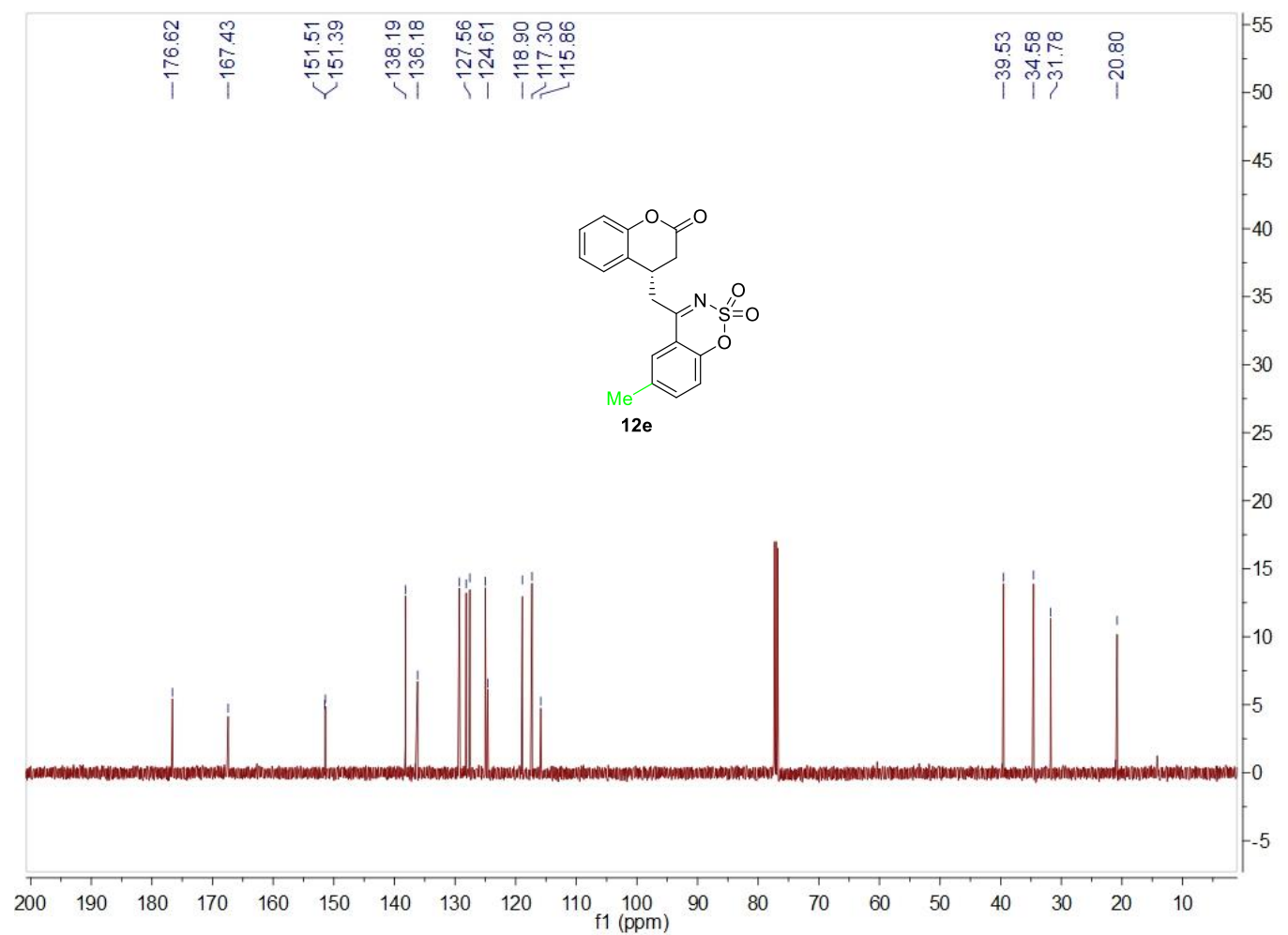

Chiral HPLC analysis of rac-12e

Chrom Type: Fixed WL Chromatogram, $260 \mathrm{~nm}$

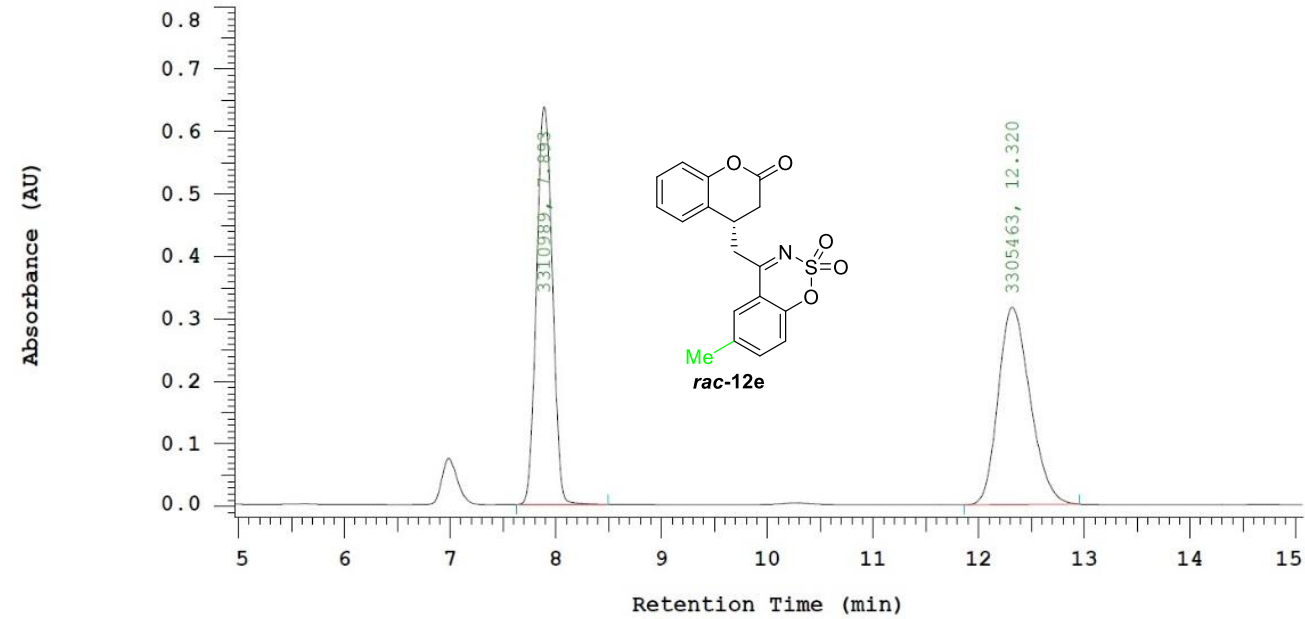

Chrom Type: Fixed WL Chromatogram, $260 \mathrm{~nm}$

Peak Quantitation: AREA

Calculation Method: AREA응

\begin{tabular}{|c|c|c|c|c|}
\hline No. & RT & Area & Area \% & $\mathrm{BC}$ \\
\hline 1 & 7.893 & 3310989 & 50.042 & $\mathrm{BB}$ \\
\hline 2 & 12.320 & 3305463 & 49.958 & $\mathrm{BB}$ \\
\hline & & 6616452 & 100.000 & \\
\hline
\end{tabular}




\section{Chiral HPLC analysis of $\mathbf{1 2 e}$}

Chrom Type: Fixed WL Chromatogram, $260 \mathrm{~nm}$

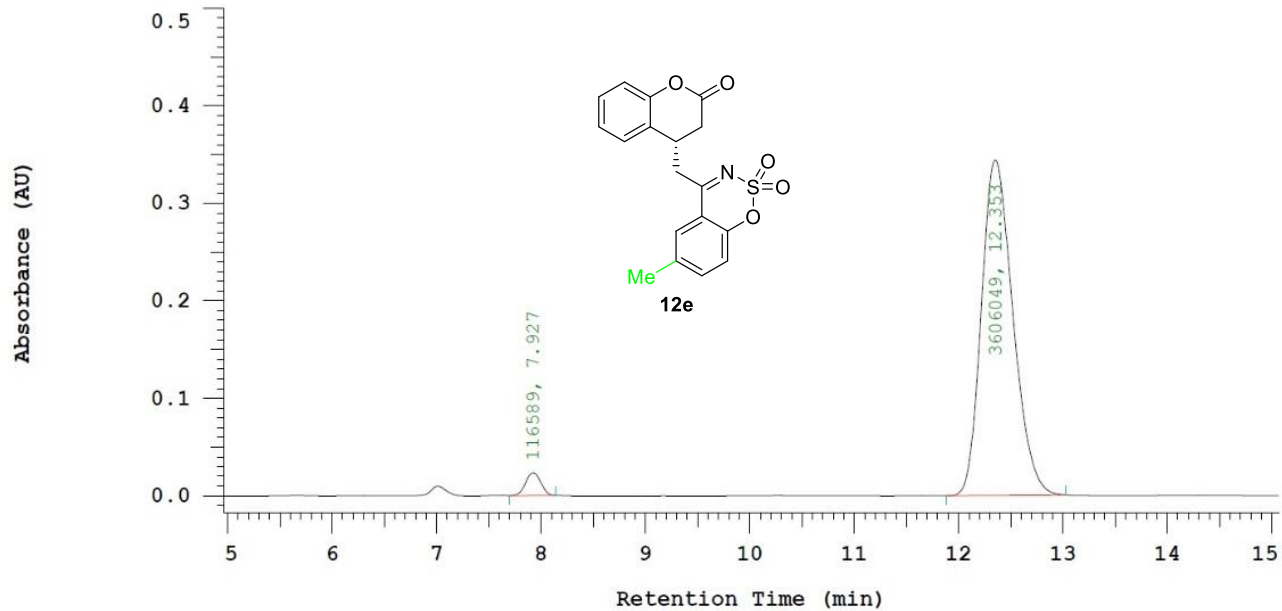

Chrom Type: Fixed WL Chromatogram, $260 \mathrm{~nm}$

Peak Quantitation: AREA

Calculation Method: AREA응

\begin{tabular}{rrrrr}
\multicolumn{1}{c}{ RT } & \multicolumn{1}{c}{ Area } & Area & BC \\
\hline 1 & 7.927 & 116589 & 3.132 & BB \\
2 & 12.353 & 3606049 & 96.868 & BB \\
\hline & & 3722638 & 100.000
\end{tabular}

\section{${ }^{1} \mathrm{H}$ NMR of $12 f, \mathrm{CDCl}_{3}$}

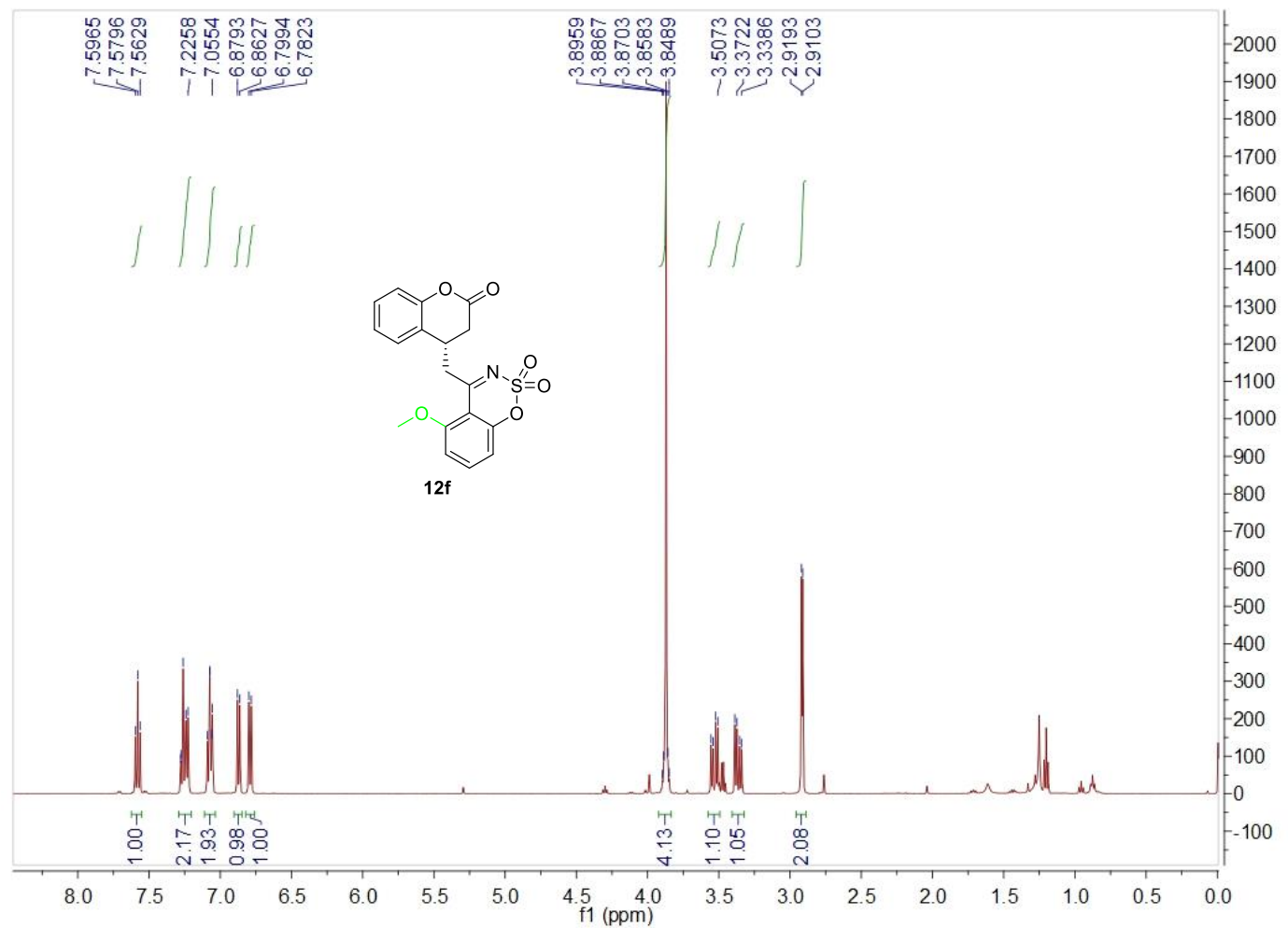


${ }^{13} \mathrm{CNMR}$ of $12 \mathrm{f}, \mathrm{CDCl}_{3}$

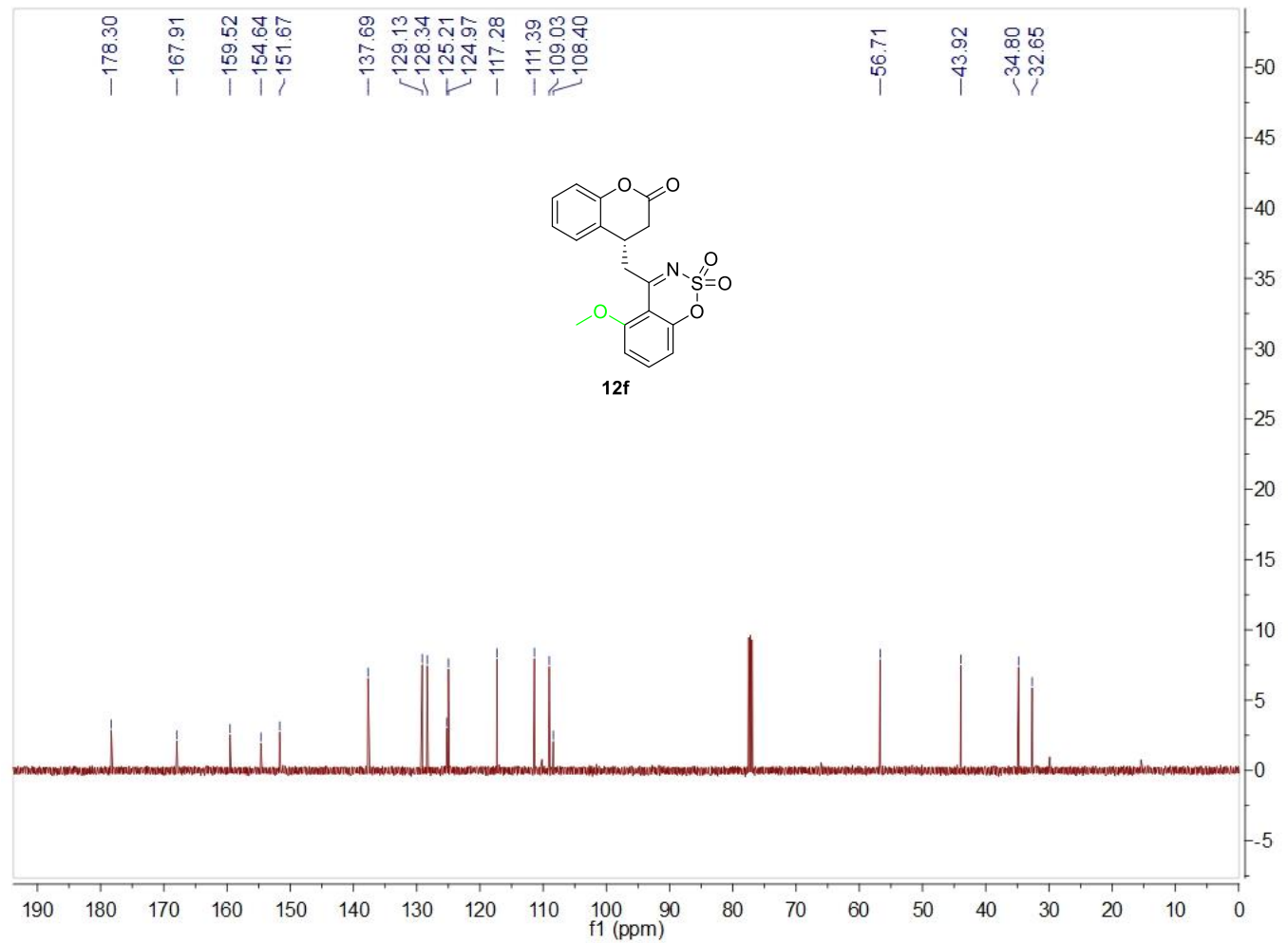

Chiral HPLC analysis of rac-12f

Chrom Type: Fixed WL Chromatogram, 299 nm

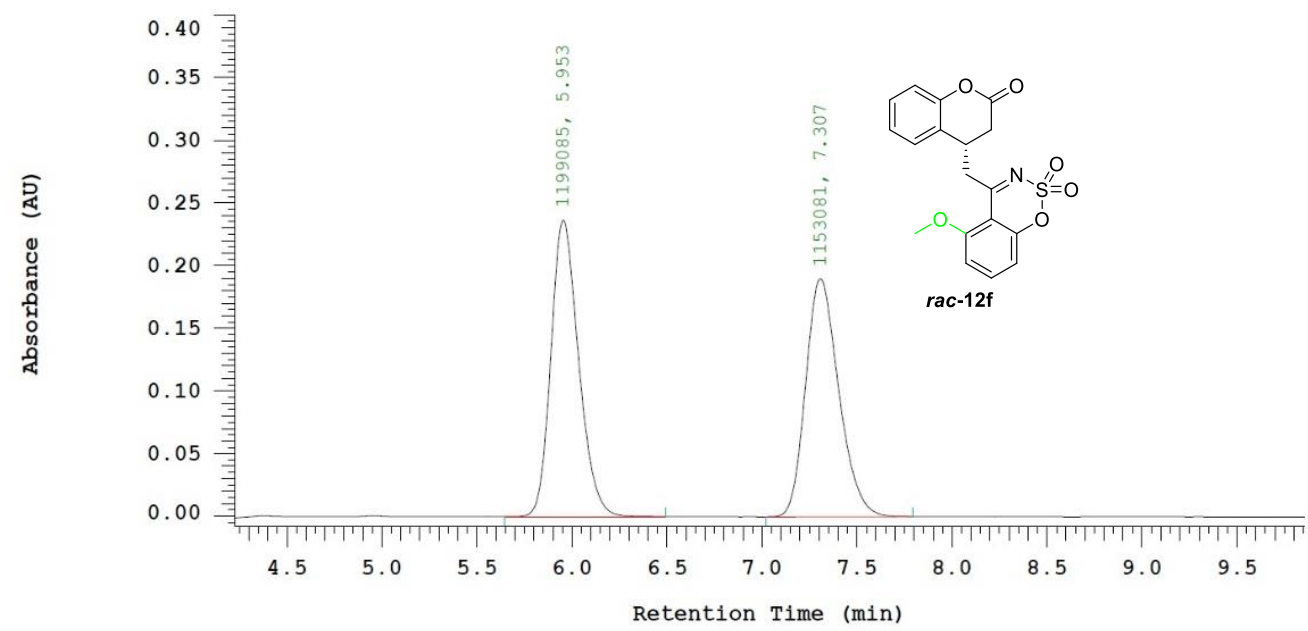

Chrom Type: Fixed WL Chromatogram, 299 nm

Peak Quantitation: AREA

Calculation Method: AREA응

\begin{tabular}{|c|c|c|c|c|}
\hline No. & $\mathrm{RT}$ & Area & Area 응 & $\mathrm{BC}$ \\
\hline 1 & 5.953 & 1199085 & 50.978 & $\mathrm{BB}$ \\
\hline 2 & 7.307 & 1153081 & 49.022 & $\mathrm{BB}$ \\
\hline & & 2352166 & 100.000 & \\
\hline
\end{tabular}




\section{Chiral HPLC analysis of $\mathbf{1 2 f}$}

Chrom Type: Fixed WL Chromatogram, 299 nm

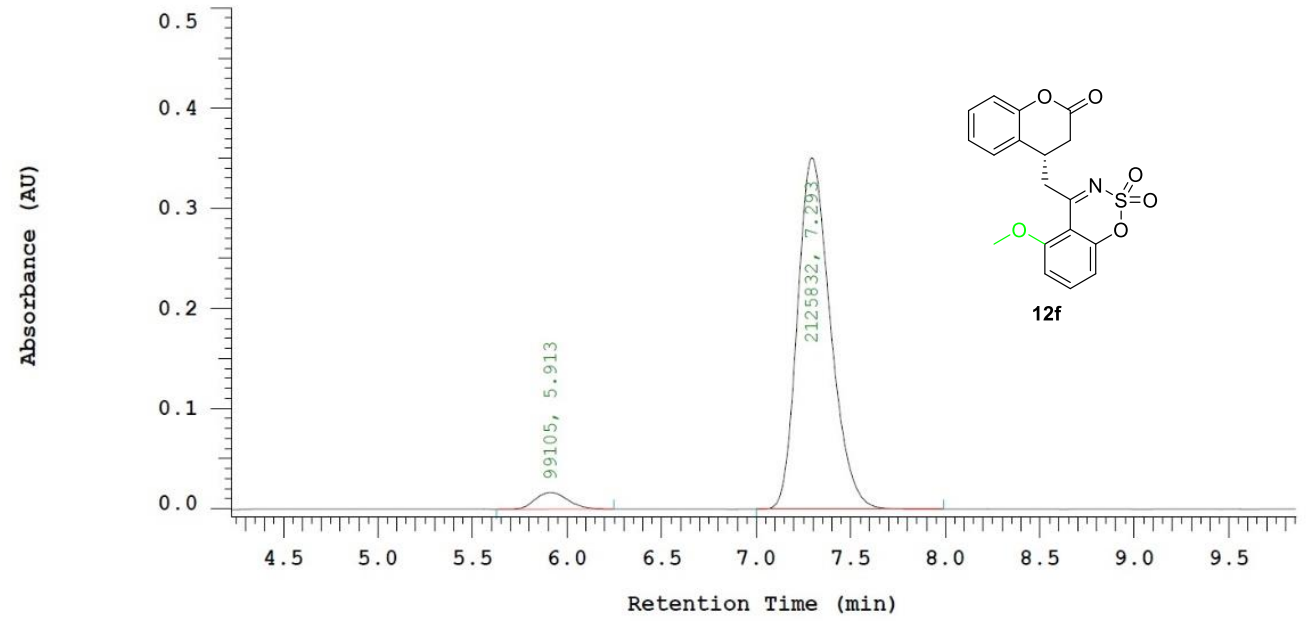

Chrom Type: Fixed WL Chromatogram, 299 nm

Peak Quantitation: AREA

Calculation Method: AREA응

\begin{tabular}{rrrrr} 
No. & RT & Area & Area & BC \\
\hline 1 & 5.913 & 99105 & 4.454 & BB \\
\hline & 7.293 & 2125832 & 95.546 & BB \\
\hline & & 2224937 & 100.000 & \\
\hline
\end{tabular}

${ }^{1} \mathrm{H}$ NMR of $12 \mathrm{~g}, \mathrm{CDCl}_{3}$

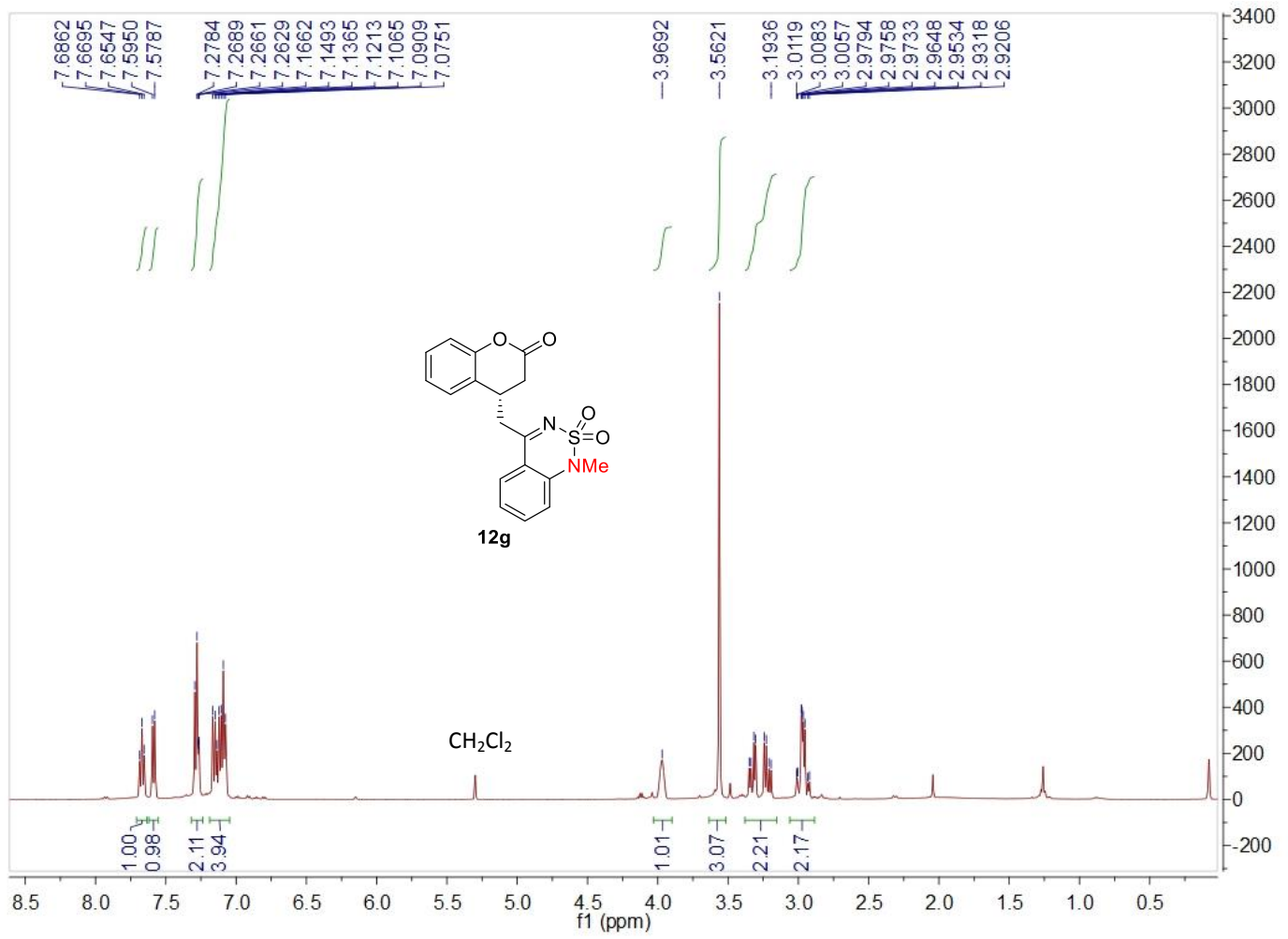


${ }^{13} \mathrm{CNMR}$ of $12 \mathrm{~g}, \mathrm{CDCl}_{3}$

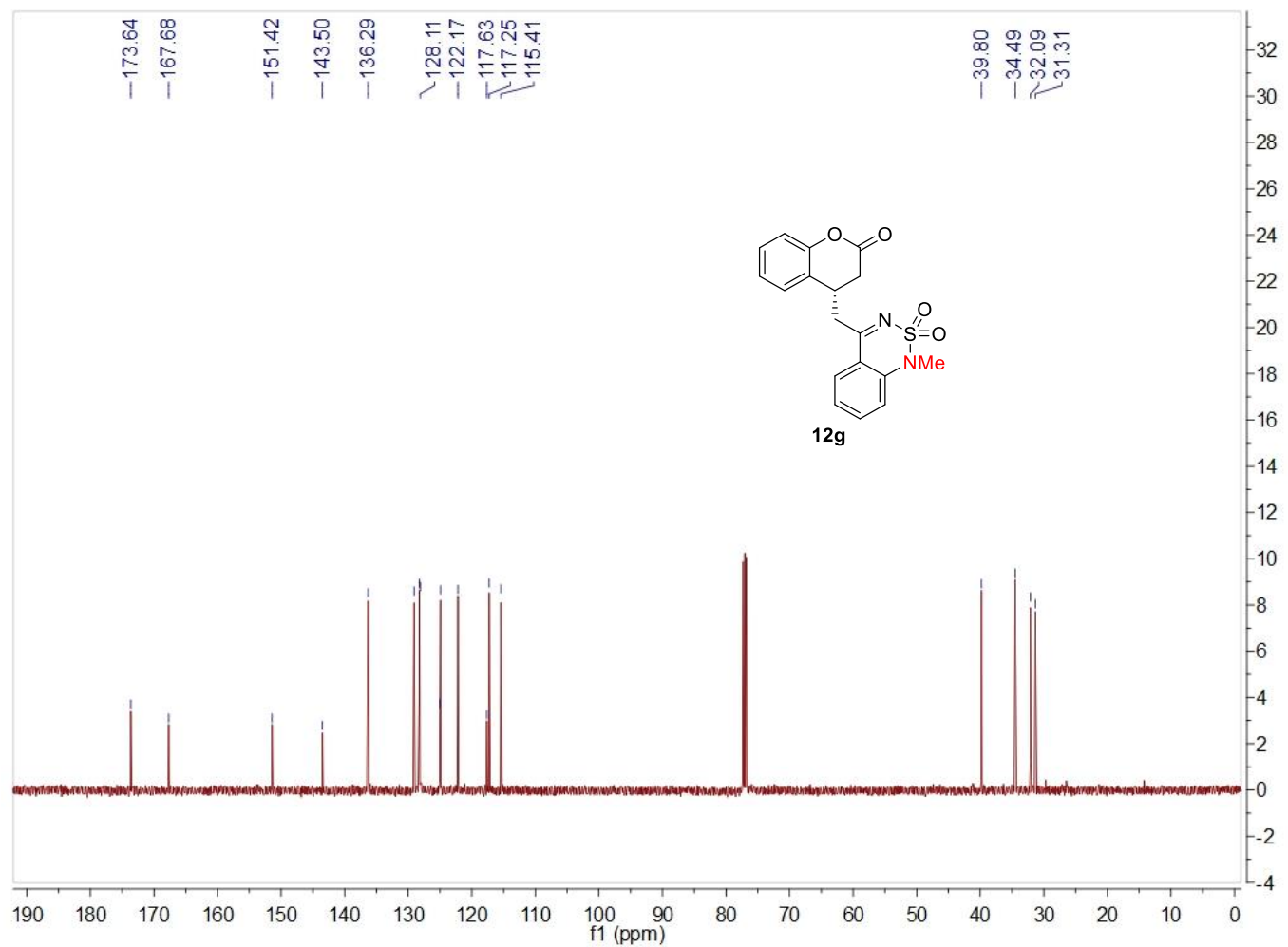

Chiral HPLC analysis of rac-12g

Chrom Type: Fixed WL Chromatogram, 225 nm

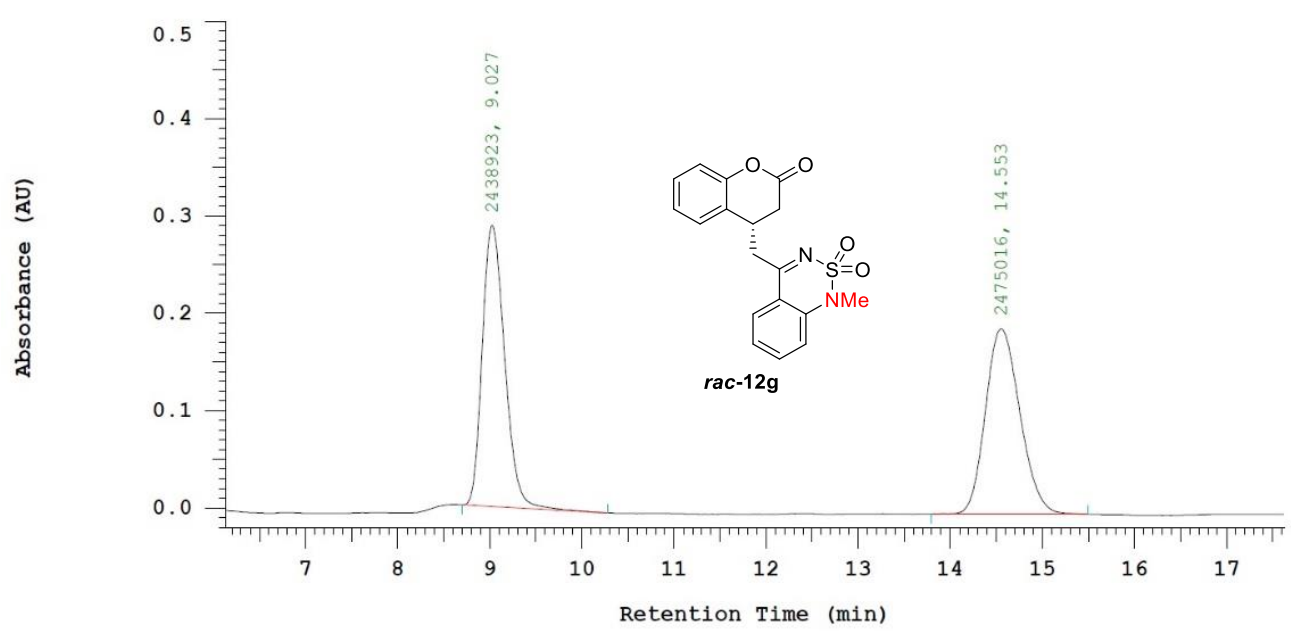

Chrom Type: Fixed WL Chromatogram, 225 nm Peak Quantitation: AREA

Calculation Method: AREA음

\begin{tabular}{|c|c|c|c|c|}
\hline No. & $\mathrm{RT}$ & Area & Area \% & $\mathrm{BC}$ \\
\hline 1 & 9.027 & 2438923 & 49.633 & $\mathrm{BB}$ \\
\hline 2 & 14.553 & 2475016 & 50.367 & $\mathrm{BB}$ \\
\hline & & 4913939 & 100.000 & \\
\hline
\end{tabular}




\section{Chiral HPLC analysis of $\mathbf{1 2 g}$}

Chrom Type: Fixed WL Chromatogram, $225 \mathrm{~nm}$

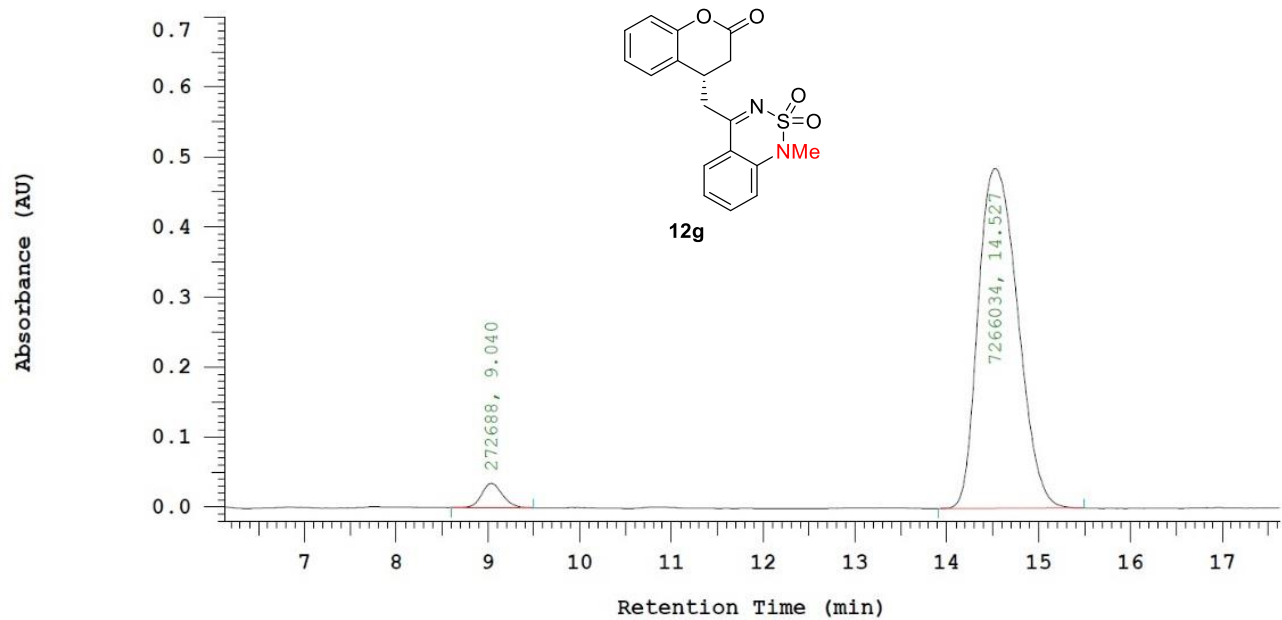

Chrom Type: Fixed WL Chromatogram, $225 \mathrm{~nm}$

Peak Quantitation: AREA

Calculation Method: AREA응

\begin{tabular}{|c|c|c|c|c|}
\hline No. & $\mathrm{RT}$ & Area & Area 옹 & $\mathrm{BC}$ \\
\hline 1 & 9.040 & 272688 & 3.617 & $\mathrm{BB}$ \\
\hline 2 & 14.527 & 7266034 & 96.383 & $\mathrm{BB}$ \\
\hline & & 7538722 & 100.000 & \\
\hline
\end{tabular}

${ }^{1} \mathrm{H}$ NMR of $12 \mathrm{~h}, \mathrm{CDCl}_{3}$

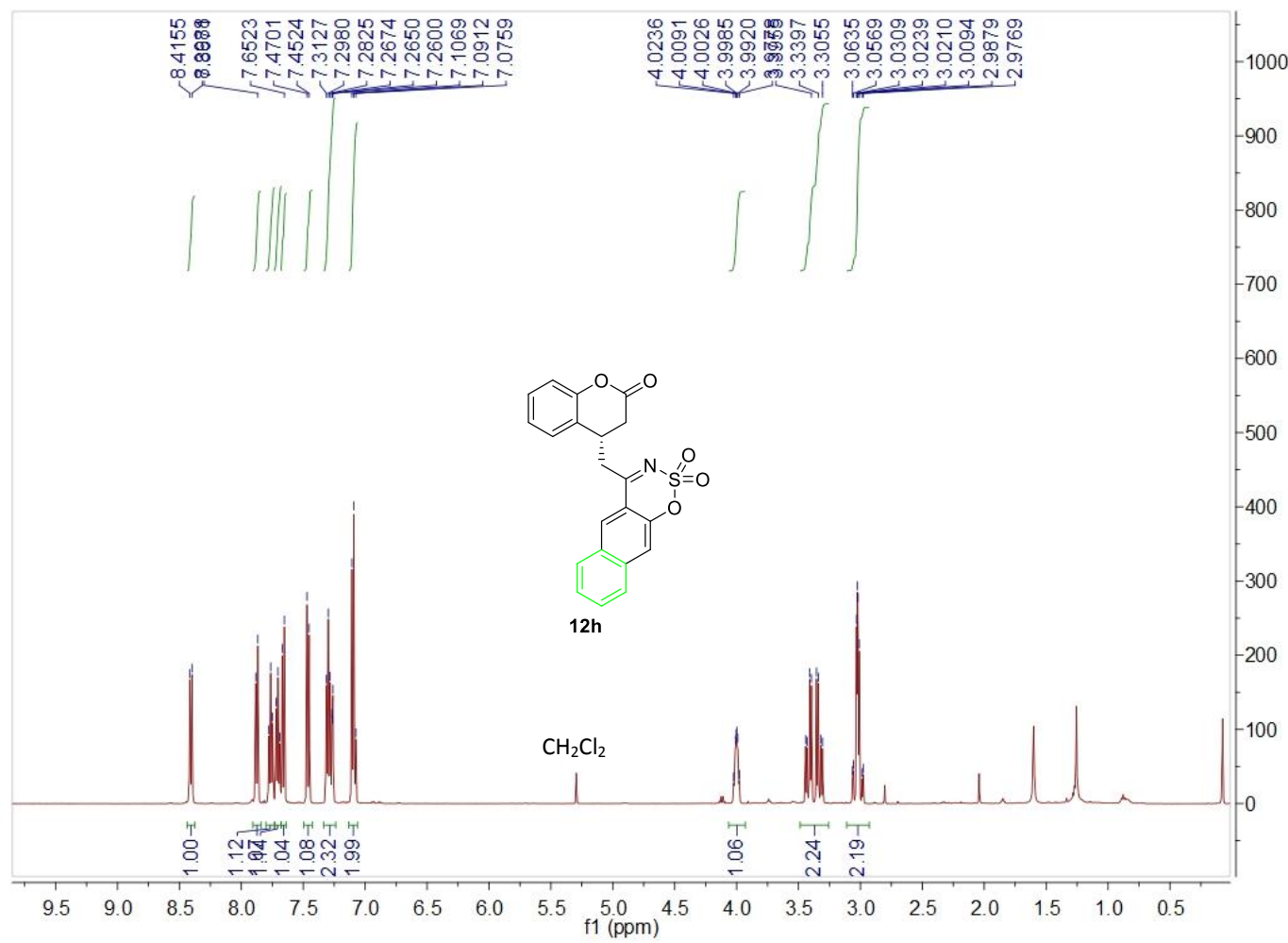


${ }^{13} \mathrm{CNMR}$ of $12 \mathrm{~h}, \mathrm{CDCl}_{3}$

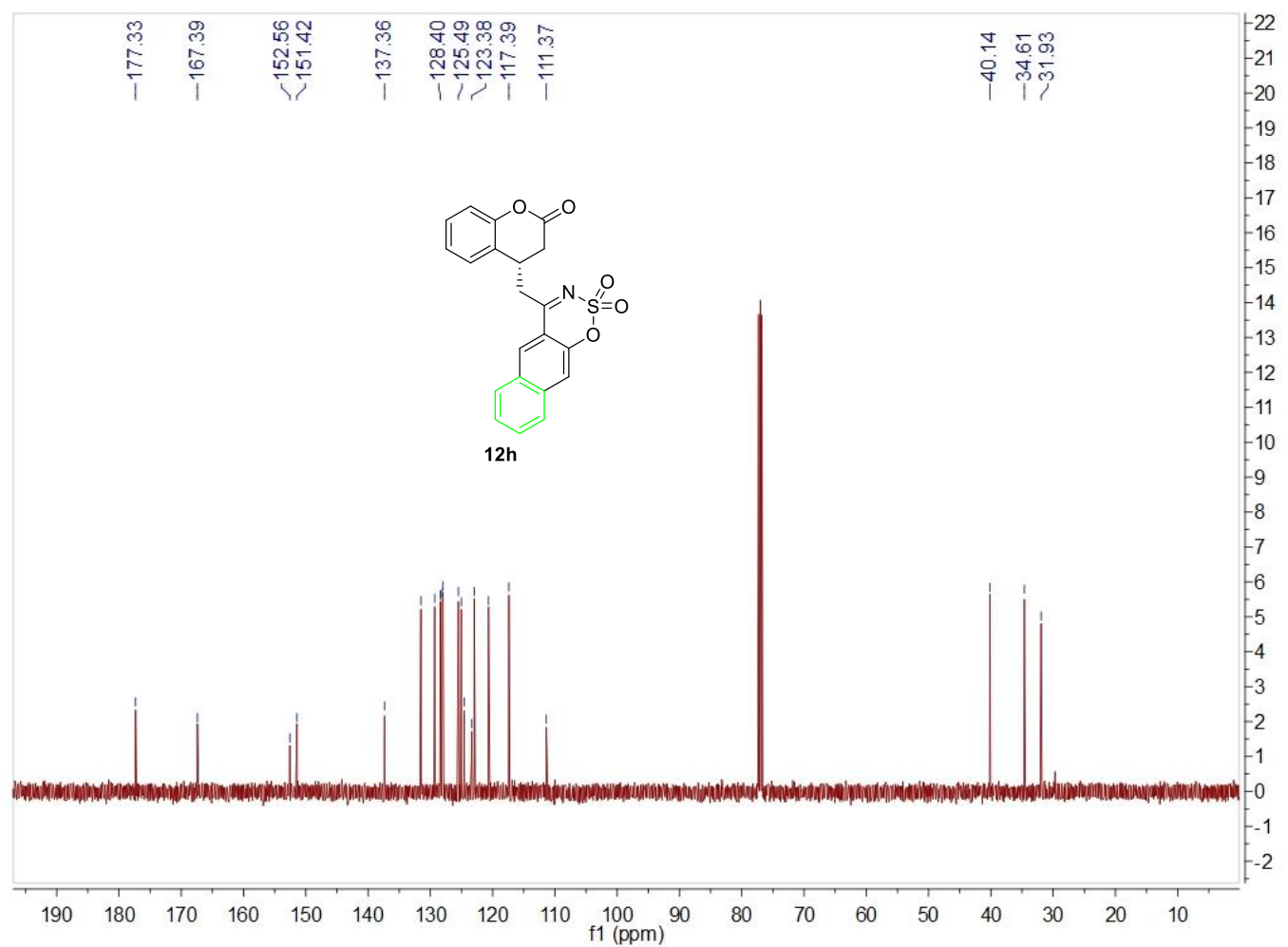

Chiral HPLC analysis of rac-12h

Chrom Type: Fixed WL Chromatogram, 260 nm

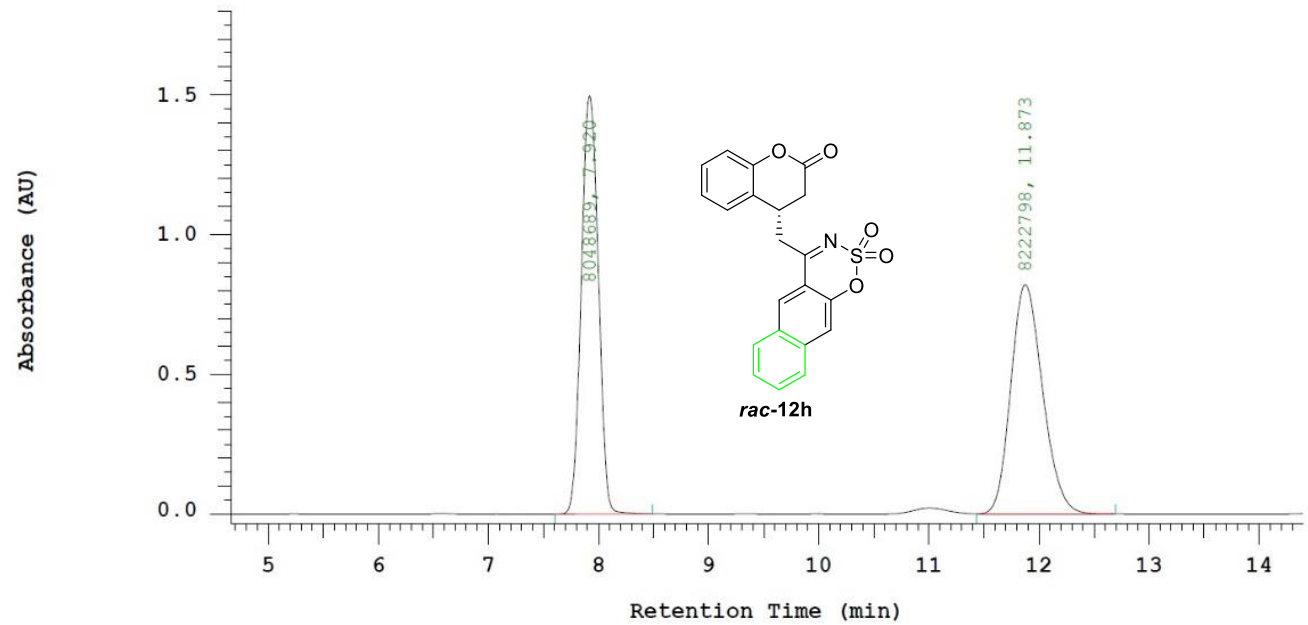

Chrom Type: Fixed WL Chromatogram, $260 \mathrm{~nm}$

Peak Quantitation: AREA

Calculation Method: AREA응

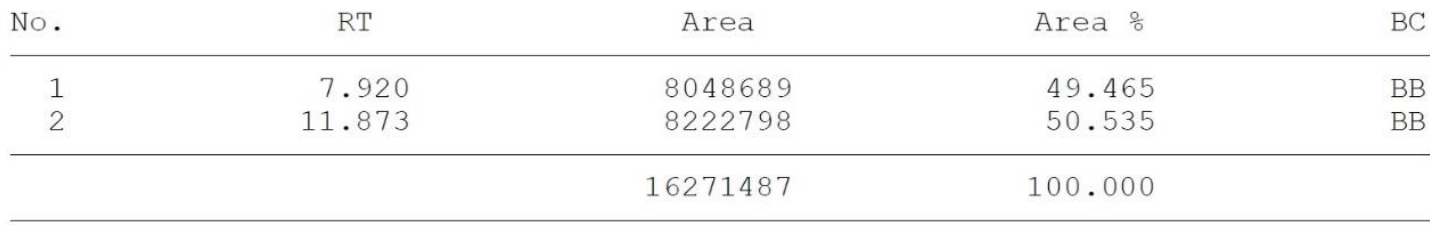




\section{Chiral HPLC analysis of $\mathbf{1 2 h}$}

Chrom Type: Fixed WL Chromatogram, 260 nm

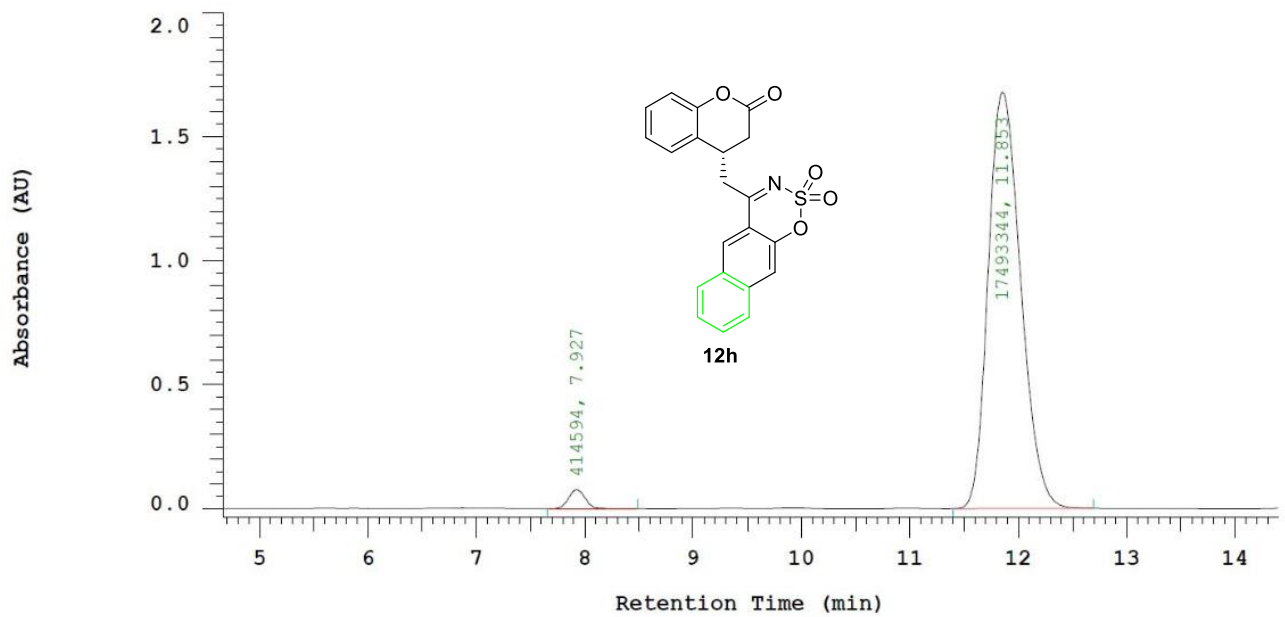

Chrom Type: Fixed WL Chromatogram, $260 \mathrm{~nm}$

Peak Quantitation: AREA

Calculation Method: AREA\%

\begin{tabular}{|c|c|c|c|}
\hline Jo. & RT & Area & Area 응 \\
\hline 1 & 7.927 & 414594 & 2.315 \\
\hline 2 & 11.853 & 17493344 & 97.685 \\
\hline & & 17907938 & 100.000 \\
\hline
\end{tabular}

${ }^{1} \mathrm{H}$ NMR of $13 a, \mathrm{CDCl}_{3}$

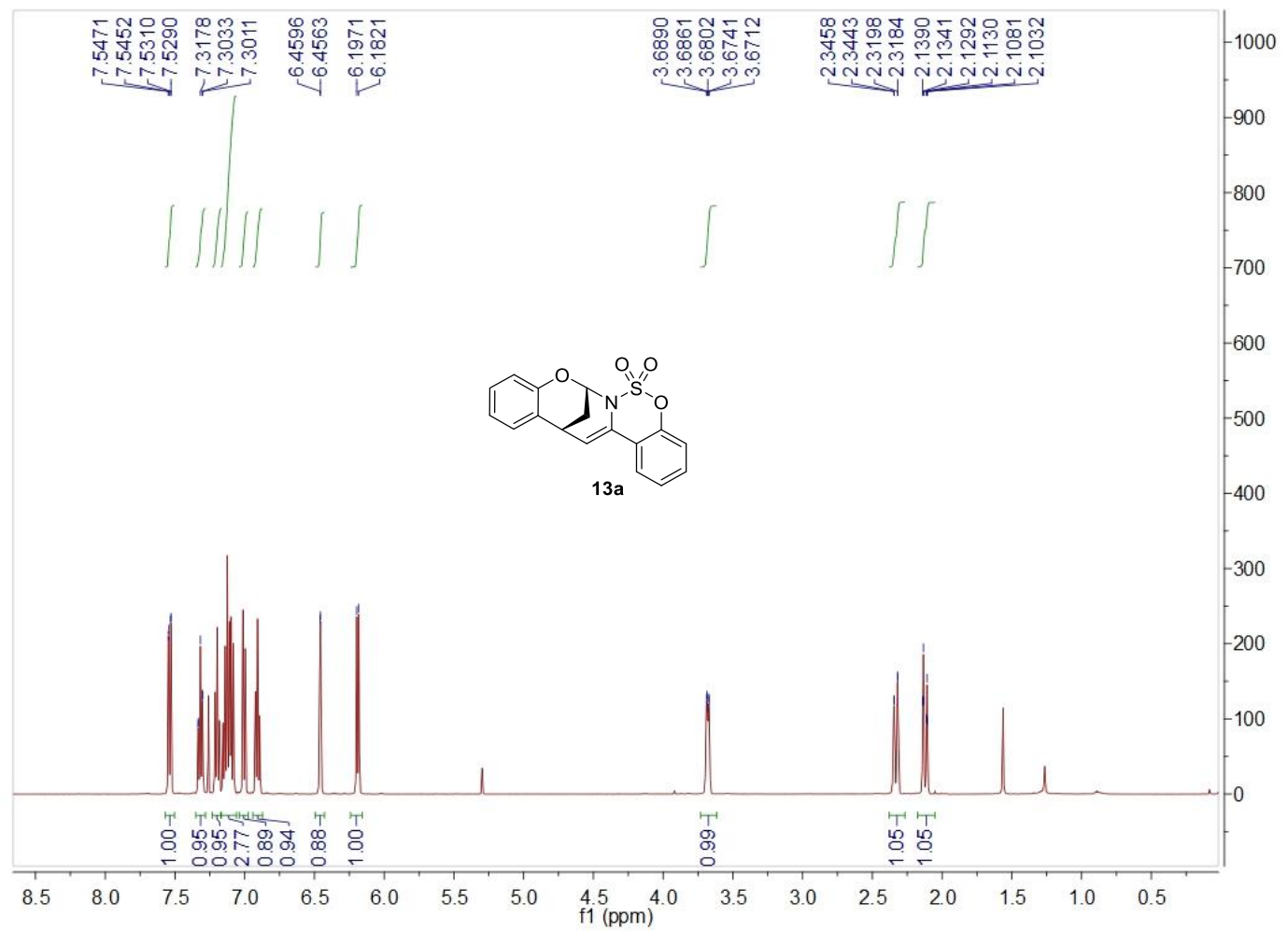


${ }^{13} \mathrm{CNMR}$ of $13 \mathrm{a}, \mathrm{CDCl}_{3}$

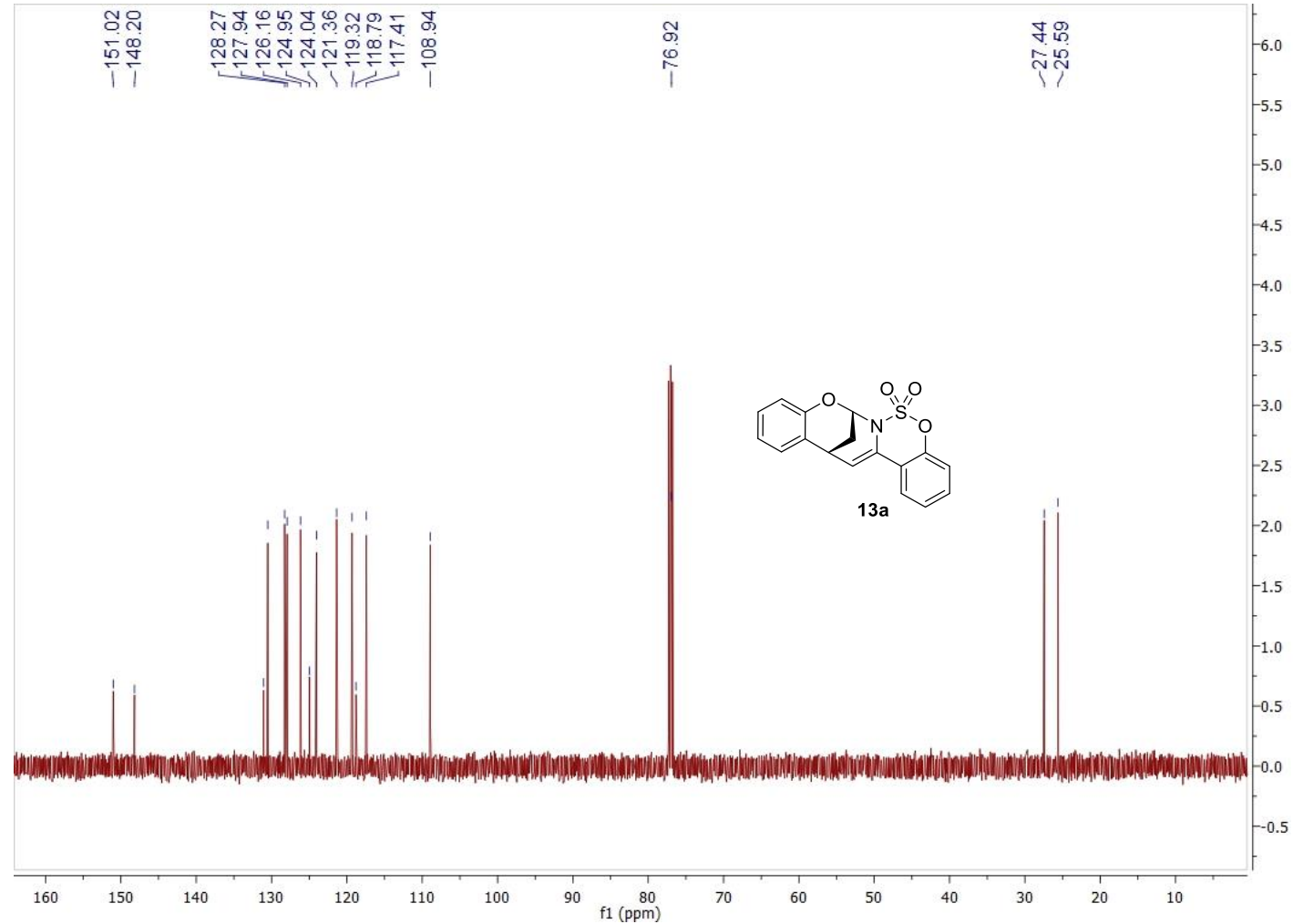

Chiral HPLC analysis of rac-13a

Chrom Type: Fixed WL Chromatogram, 216 nm

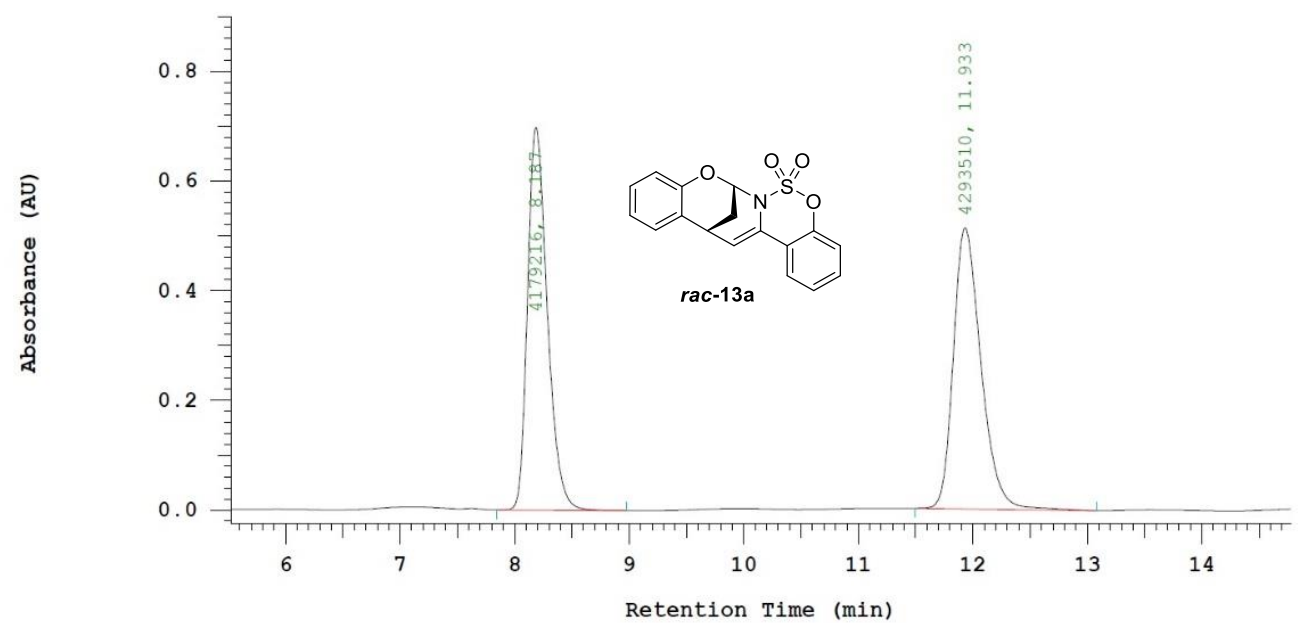

Chrom Type: Fixed WL Chromatogram, $216 \mathrm{~nm}$

Peak Quantitation: AREA

Calculation Method: AREA응

\begin{tabular}{rrrrr}
\multicolumn{1}{c}{ RT } & Area & Area & BC \\
\hline 1 & 8.187 & 4179216 & 49.326 & BB \\
11.933 & 4293510 & 50.674 & BB \\
\hline & & 8472726 & 100.000 &
\end{tabular}




\section{Chiral HPLC analysis of 13a}

Chrom Type: Fixed WL Chromatogram, 216 nm

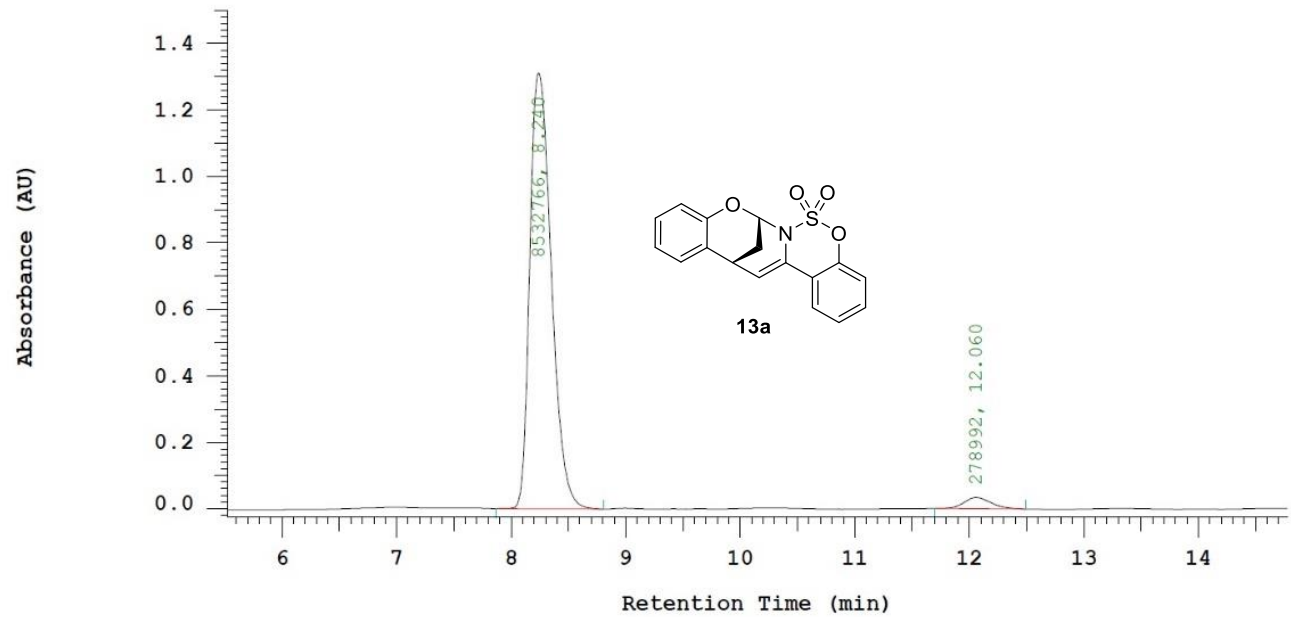

Chrom Type: Fixed WL Chromatogram, $216 \mathrm{~nm}$

Peak Quantitation: AREA

Calculation Method: AREA응

\begin{tabular}{rrrr}
\multicolumn{1}{c}{ RT } & \multicolumn{1}{c}{ Area } & Area $\%$ & BC \\
\hline 1 & 8.240 & 8532766 & 96.834 \\
BB \\
\hline
\end{tabular}

${ }^{1} \mathrm{H}$ NMR of $13 b, \mathrm{CDCl}_{3}$

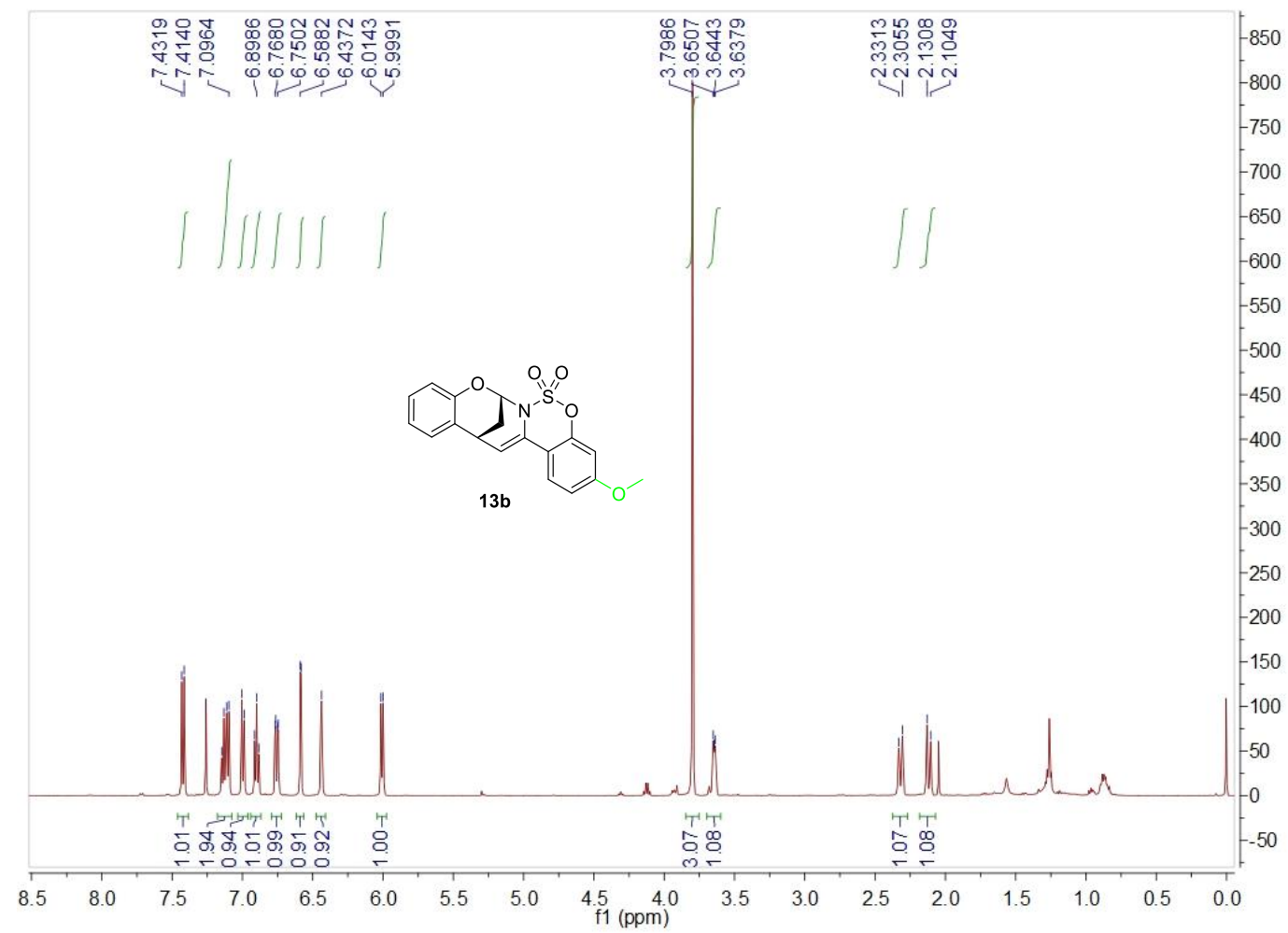


DEPT-Q of $13 \mathbf{b}, \mathrm{CDCl}_{3}$

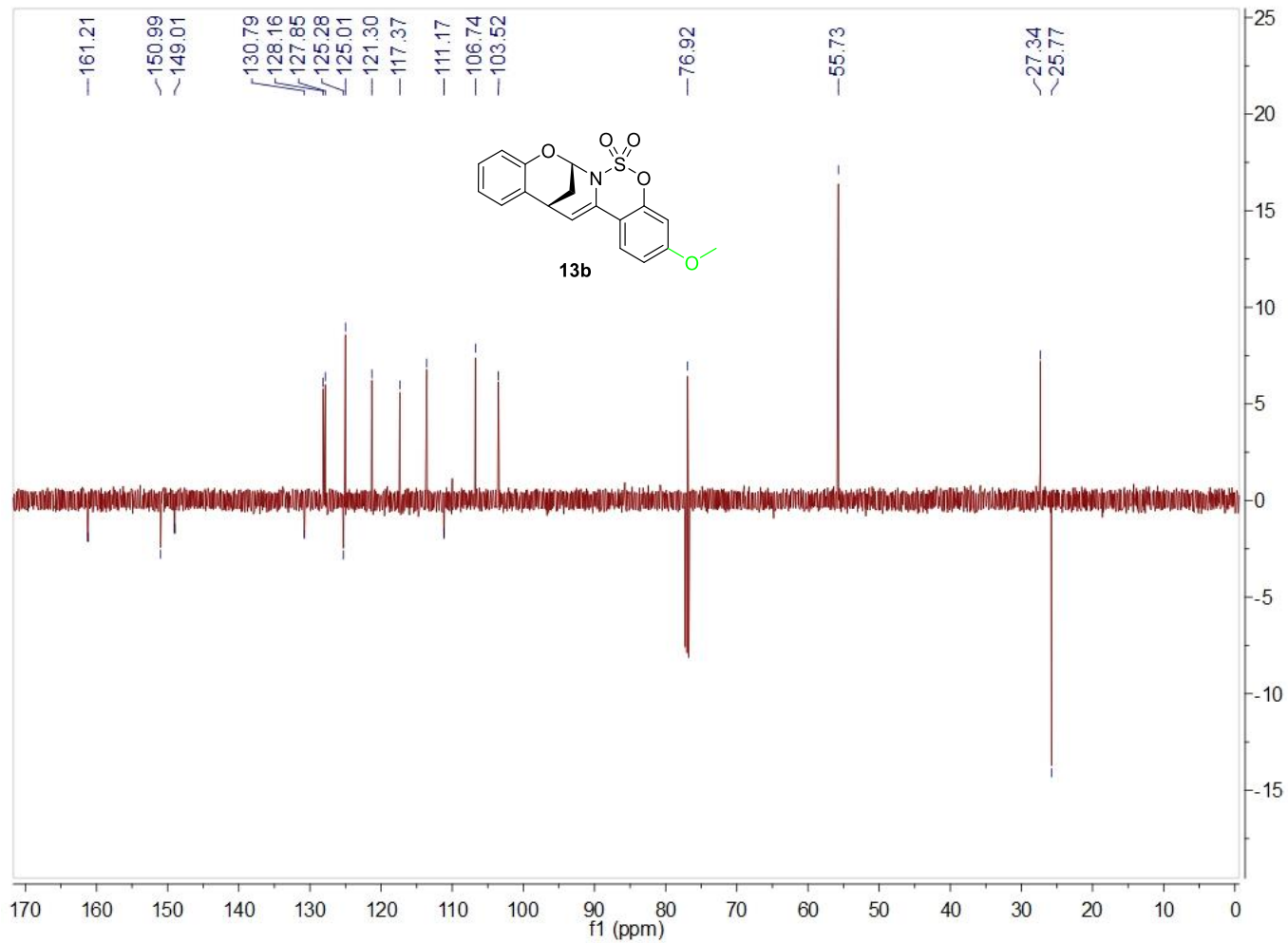

Chiral HPLC analysis of rac-13b

Chrom Type: Fixed WL Chromatogram, $210 \mathrm{~nm}$

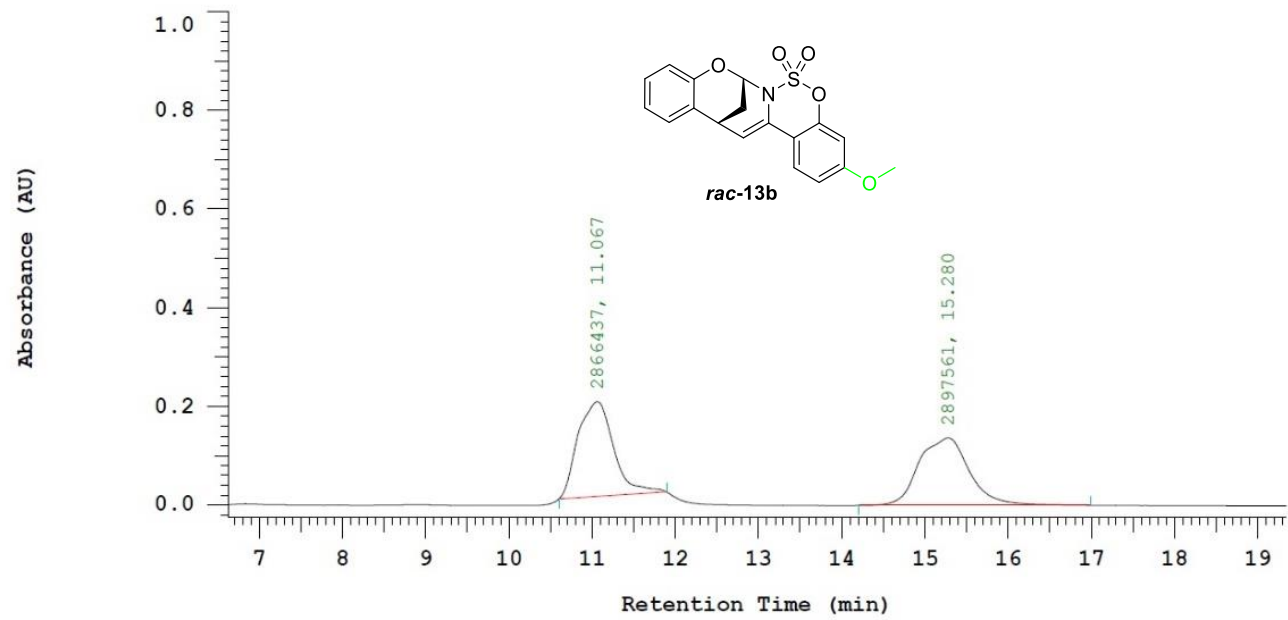

Chrom Type: Fixed WL Chromatogram, $210 \mathrm{~nm}$

Peak Quantitation: AREA

Calculation Method: AREA음

\begin{tabular}{ccccc} 
No. & RT & Area & Area \% & BC \\
\hline 1 & 11.067 & 2866437 & 49.730 & BB \\
2 & 15.280 & 2897561 & 50.270 & BB \\
\hline & 5763998 & 100.000 & \\
\hline
\end{tabular}




\section{Chiral HPLC analysis of 13b}

Chrom Type: Fixed WL Chromatogram, 210 nm

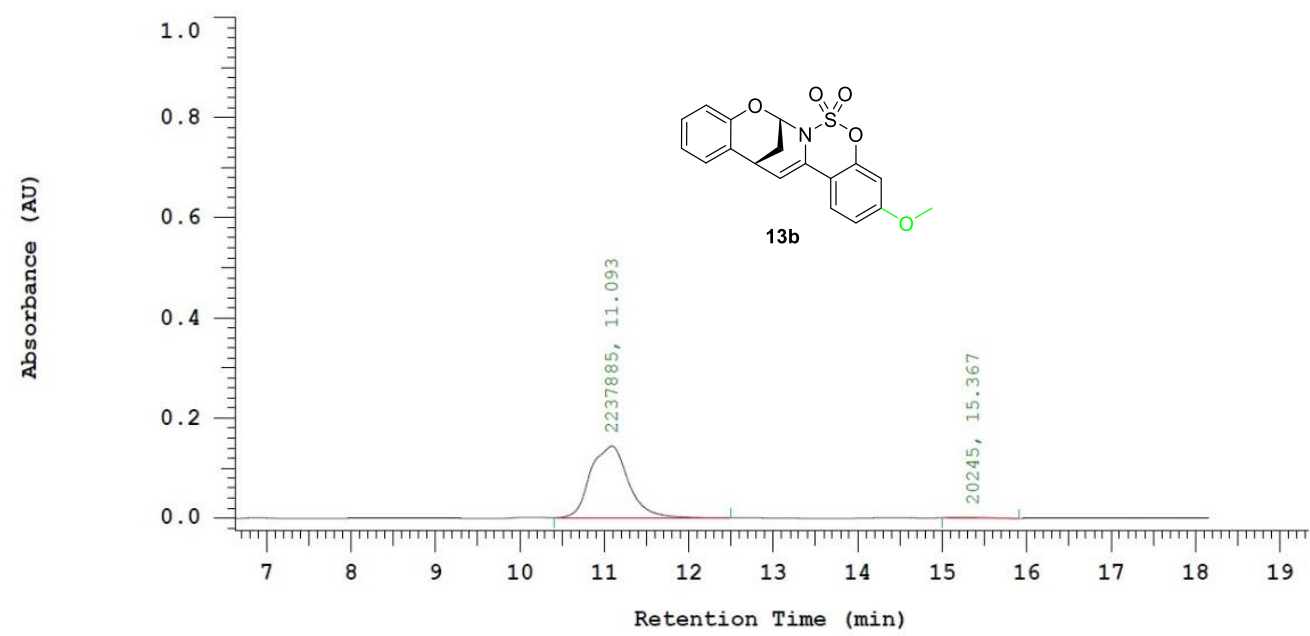

Chrom Type: Fixed WL Chromatogram, $210 \mathrm{~nm}$

Peak Quantitation: AREA

Calculation Method: AREA응

\begin{tabular}{|c|c|c|c|c|}
\hline No. & $\mathrm{RT}$ & Area & Area $\frac{\circ}{b}$ & $\mathrm{BC}$ \\
\hline 1 & 11.093 & 2237885 & 99.103 & $\mathrm{BB}$ \\
\hline 2 & 15.367 & 20245 & 0.897 & $\mathrm{BB}$ \\
\hline & & 2258130 & 100.000 & \\
\hline
\end{tabular}

${ }^{1} \mathrm{H}$ NMR of $13 \mathrm{c}, \mathrm{CDCl}_{3}$

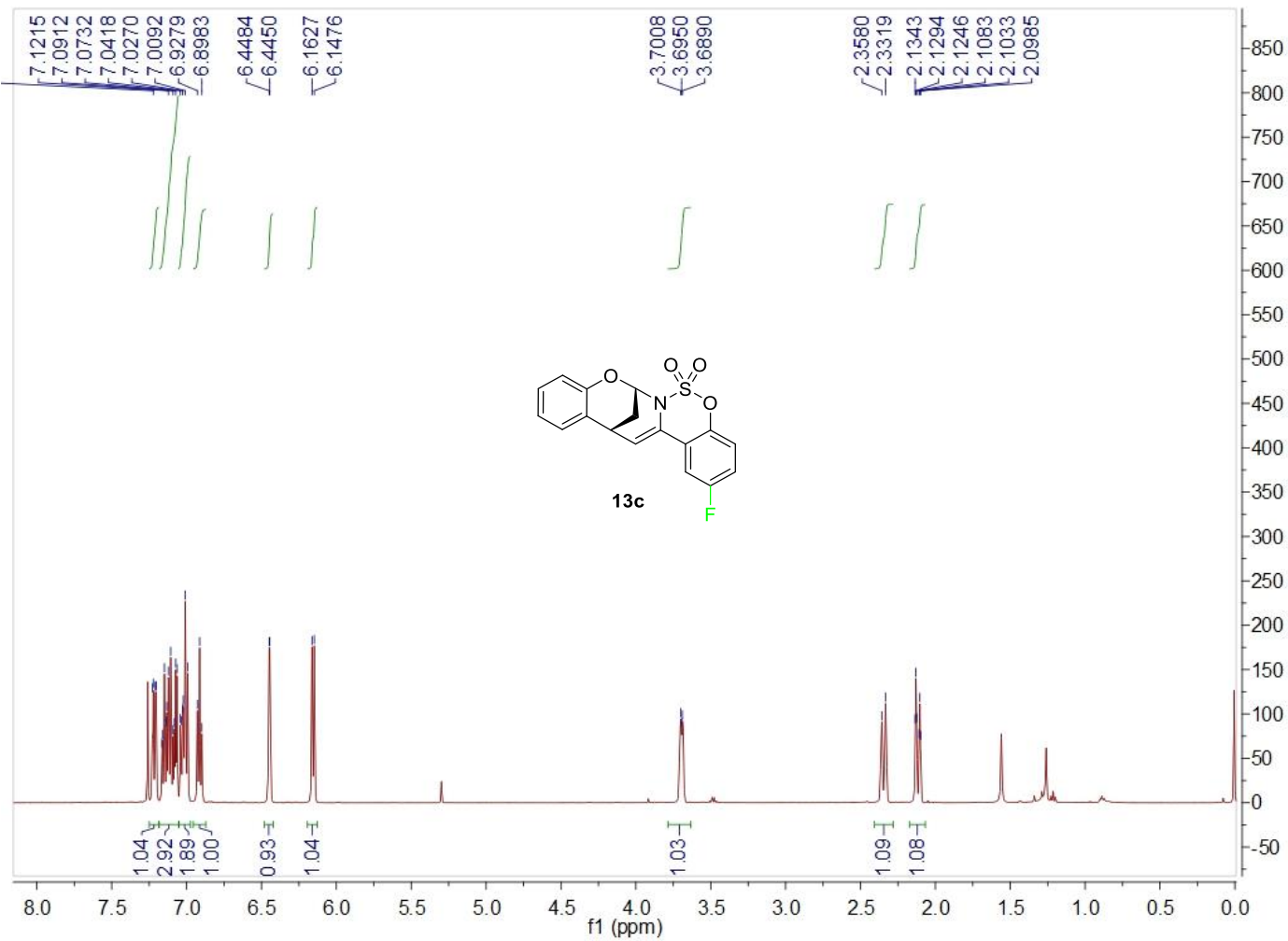


${ }^{13} \mathrm{C} \mathrm{NMR}$ of $13 \mathrm{c}, \mathrm{CDCl}_{3}$

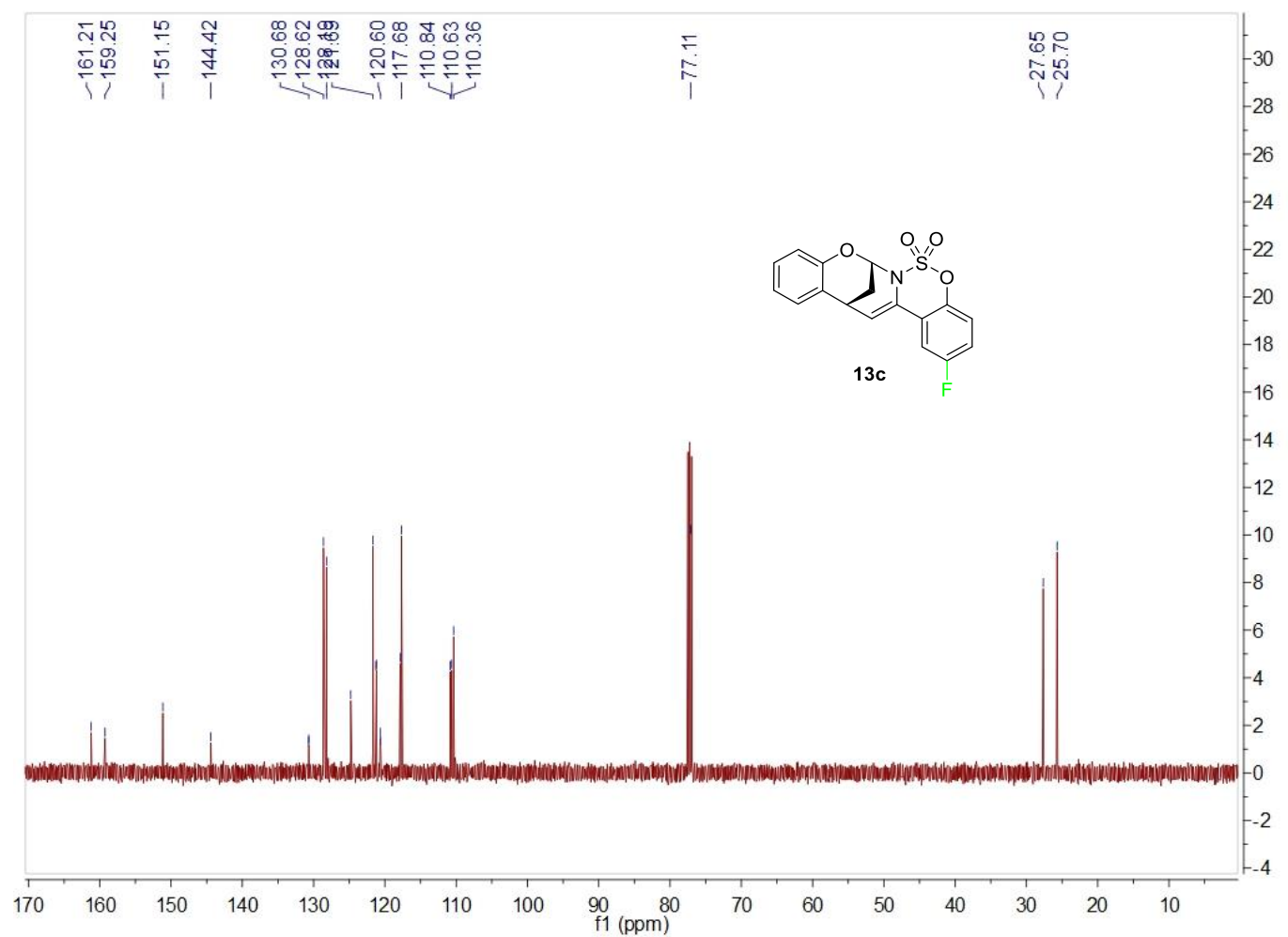

Chiral HPLC analysis of rac-13c

Chrom Type: Fixed WL Chromatogram, 210 nm

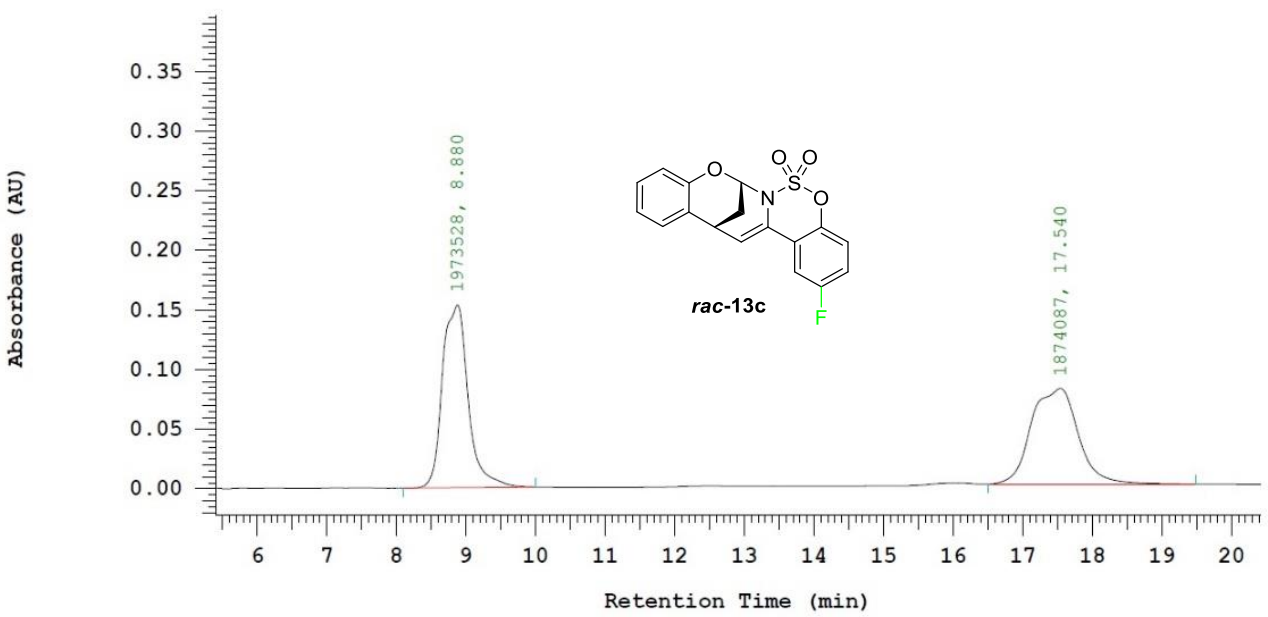

Chrom Type: Fixed WL Chromatogram, $210 \mathrm{~nm}$

Peak Quantitation: AREA

Calculation Method: AREA응

\begin{tabular}{|c|c|c|c|}
\hline No. & $\mathrm{RT}$ & Area & Area 응 \\
\hline 1 & 8.880 & 1973528 & 51.292 \\
\hline 2 & 17.540 & 1874087 & 48.708 \\
\hline & & 3847615 & 100.000 \\
\hline
\end{tabular}




\section{Chiral HPLC analysis of 13c}

Chrom Type: Fixed WL Chromatogram, 210 nm

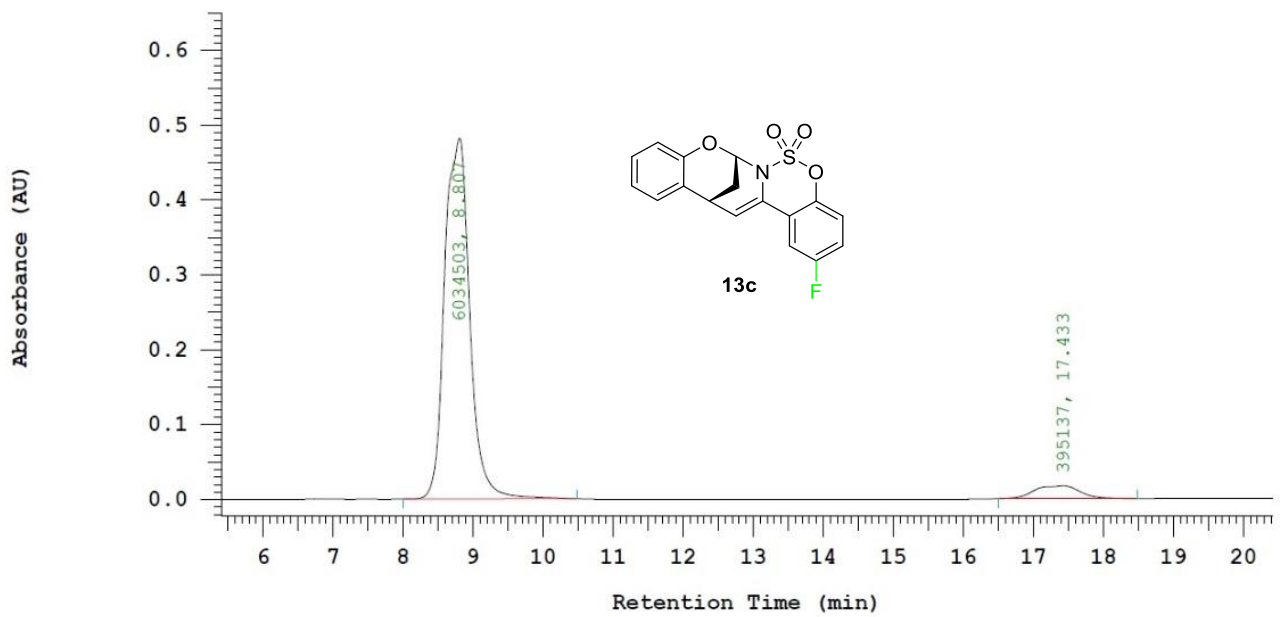

Chrom Type: Fixed WL Chromatogram, $210 \mathrm{~nm}$

Peak Quantitation: AREA

Calculation Method: AREA응

\begin{tabular}{rrrrr} 
No. & \multicolumn{1}{c}{ RT } & Area & Area $\frac{\circ}{8}$ & BC \\
\hline 1 & 8.807 & 6034503 & 93.854 & BB \\
2 & 17.433 & 395137 & 6.146 & BB \\
\hline & & 6429640 & 100.000
\end{tabular}

${ }^{1} \mathrm{H}$ NMR of $13 \mathrm{~d}, \mathrm{CDCl}_{3}$

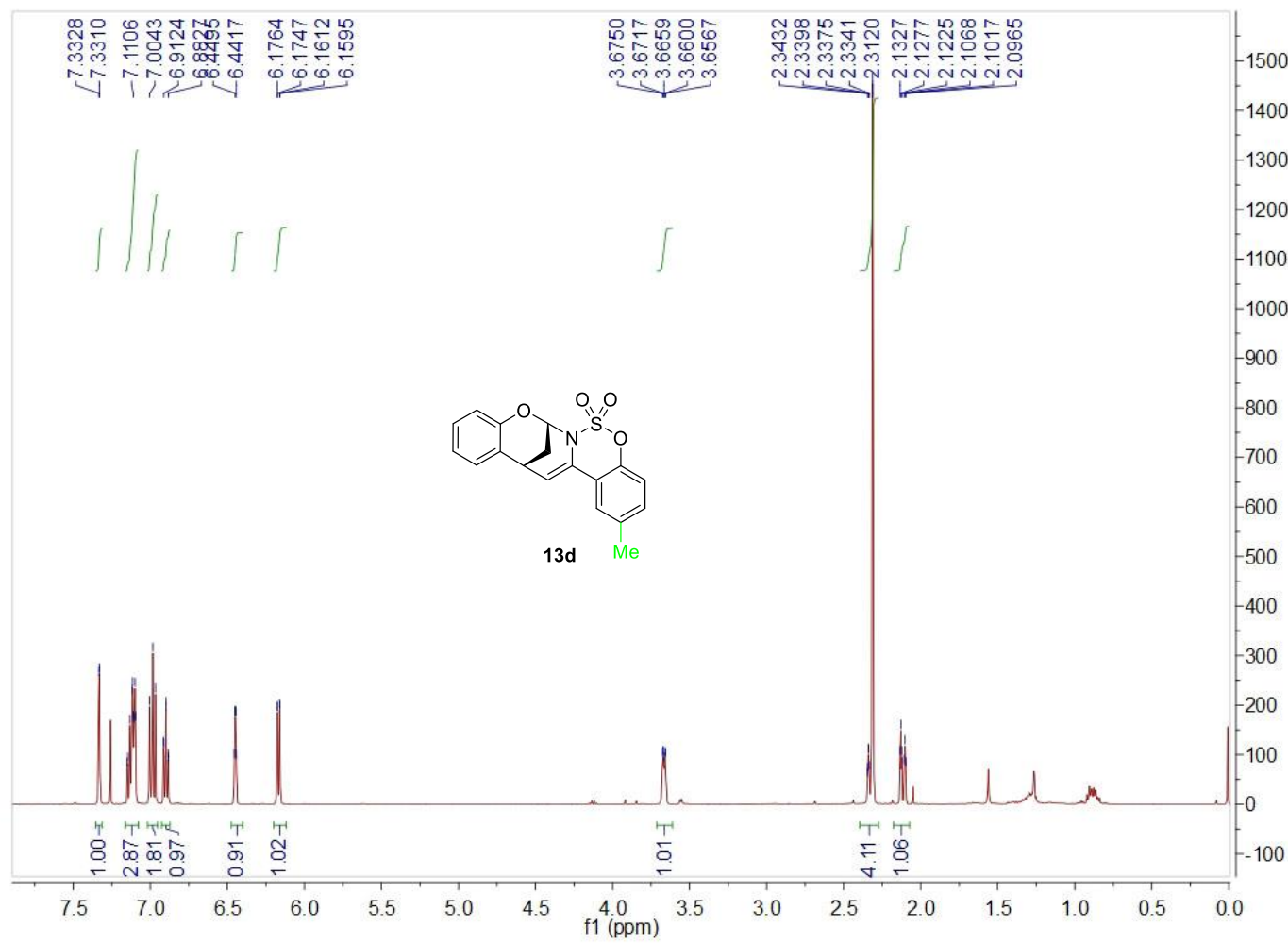


${ }^{13} \mathrm{CNMR}$ of $13 \mathrm{~d}, \mathrm{CDCl}_{3}$

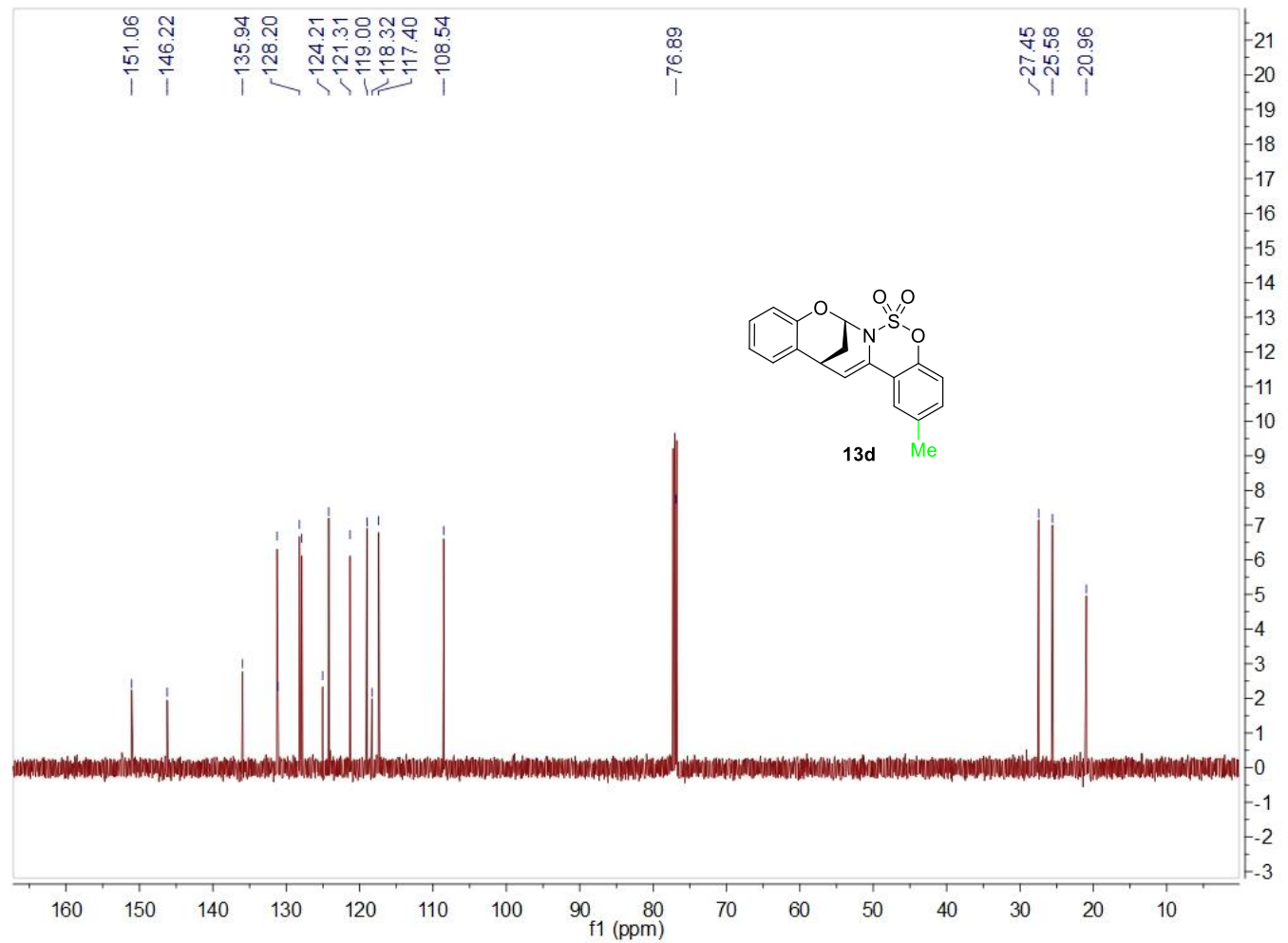

Chiral HPLC analysis of rac-13d

Chrom Type: Fixed WL Chromatogram, 216 nm

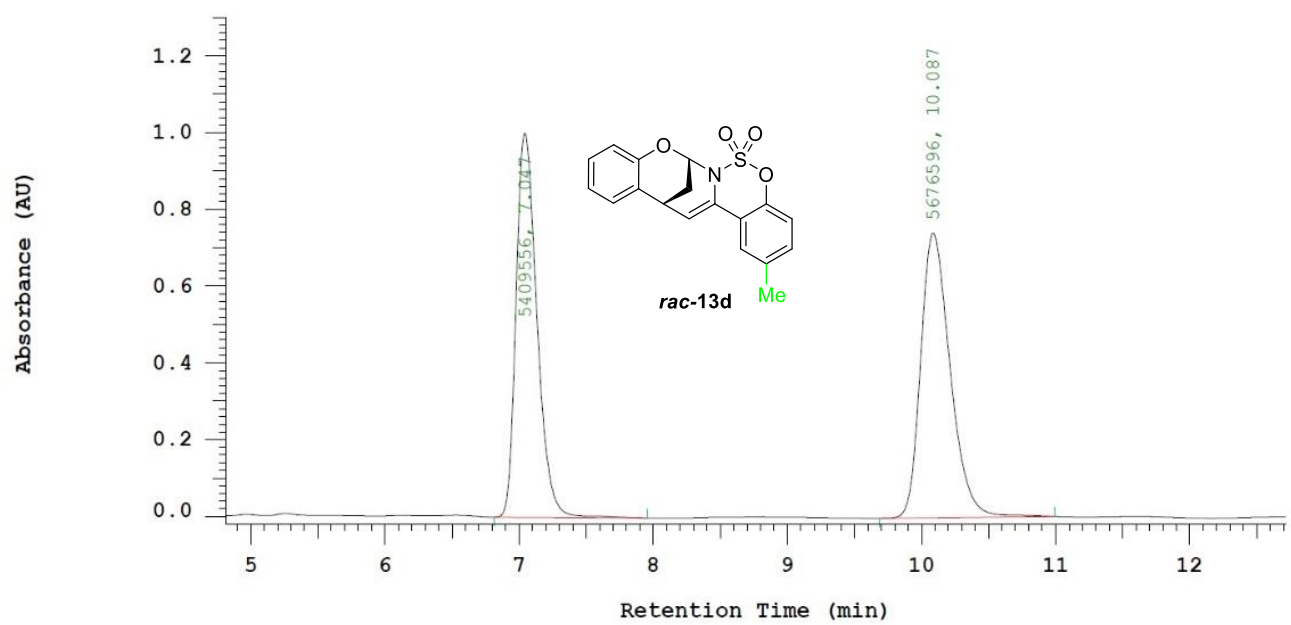

Chrom Type: Fixed WL Chromatogram, $216 \mathrm{~nm}$

Peak Quantitation: AREA

Calculation Method: AREA응

\begin{tabular}{rrrrr} 
No. & \multicolumn{1}{c}{ RT } & Area & Area & BC \\
\hline 1 & 7.047 & 5409556 & 48.796 & BB \\
\hline & 10.087 & 5676596 & 51.204 & BB \\
\hline
\end{tabular}




\section{Chiral HPLC analysis of 13d}

Chrom Type: Fixed WL Chromatogram, $216 \mathrm{~nm}$

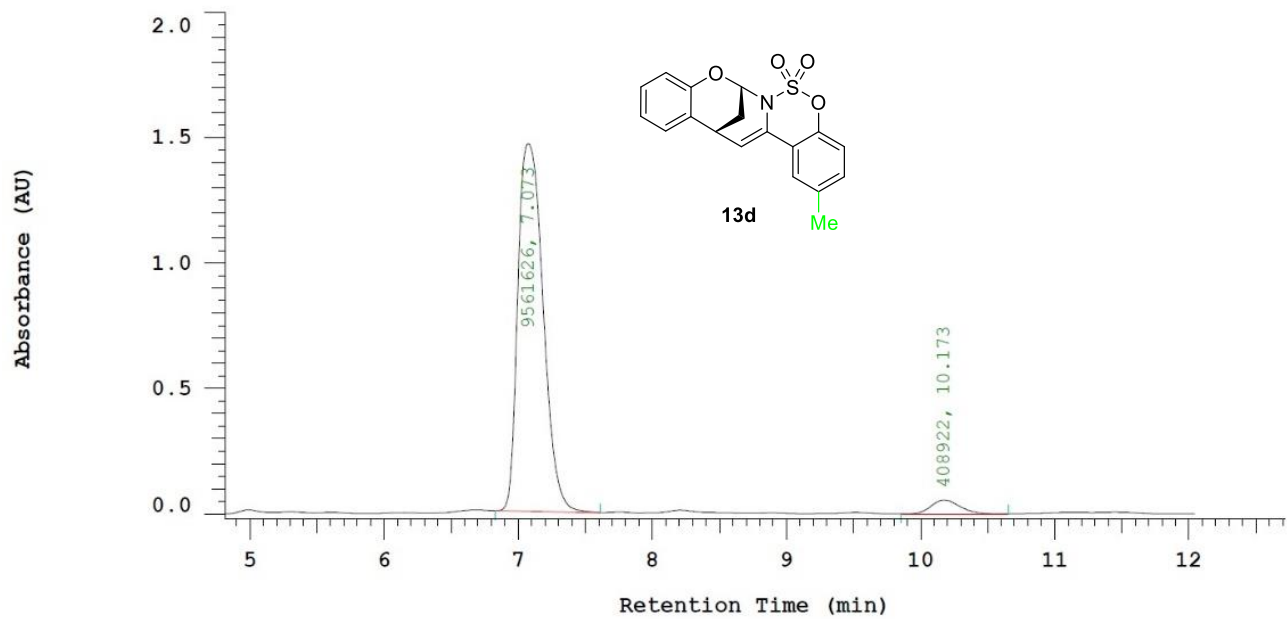

Chrom Type: Fixed WL Chromatogram, $216 \mathrm{~nm}$

Peak Ouantitation: AREA

Calculation Method: AREA응

\begin{tabular}{rrrrr} 
No. & \multicolumn{1}{c}{ RT } & Area & Area & BC \\
\hline 1 & 7.073 & 9561626 & 95.899 & BB \\
2 & 10.173 & 408922 & 4.101 & $\mathrm{BB}$ \\
\hline & & 9970548 & 100.000 \\
\hline
\end{tabular}

${ }^{1} \mathrm{H}$ NMR of $13 e, \mathrm{CDCl}_{3}$

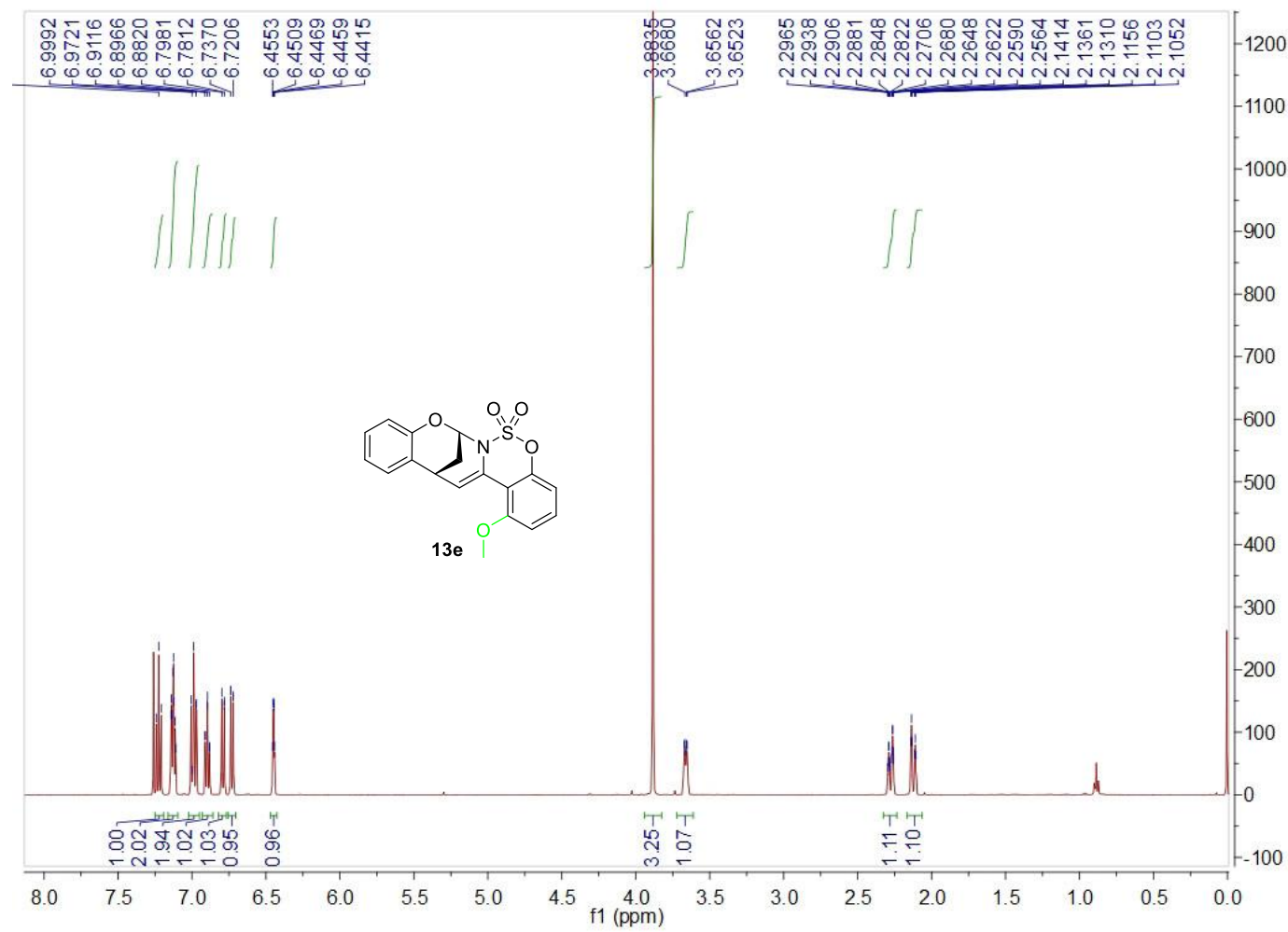


${ }^{13} \mathrm{C}$ NMR of $13 e, \mathrm{CDCl}_{3}$

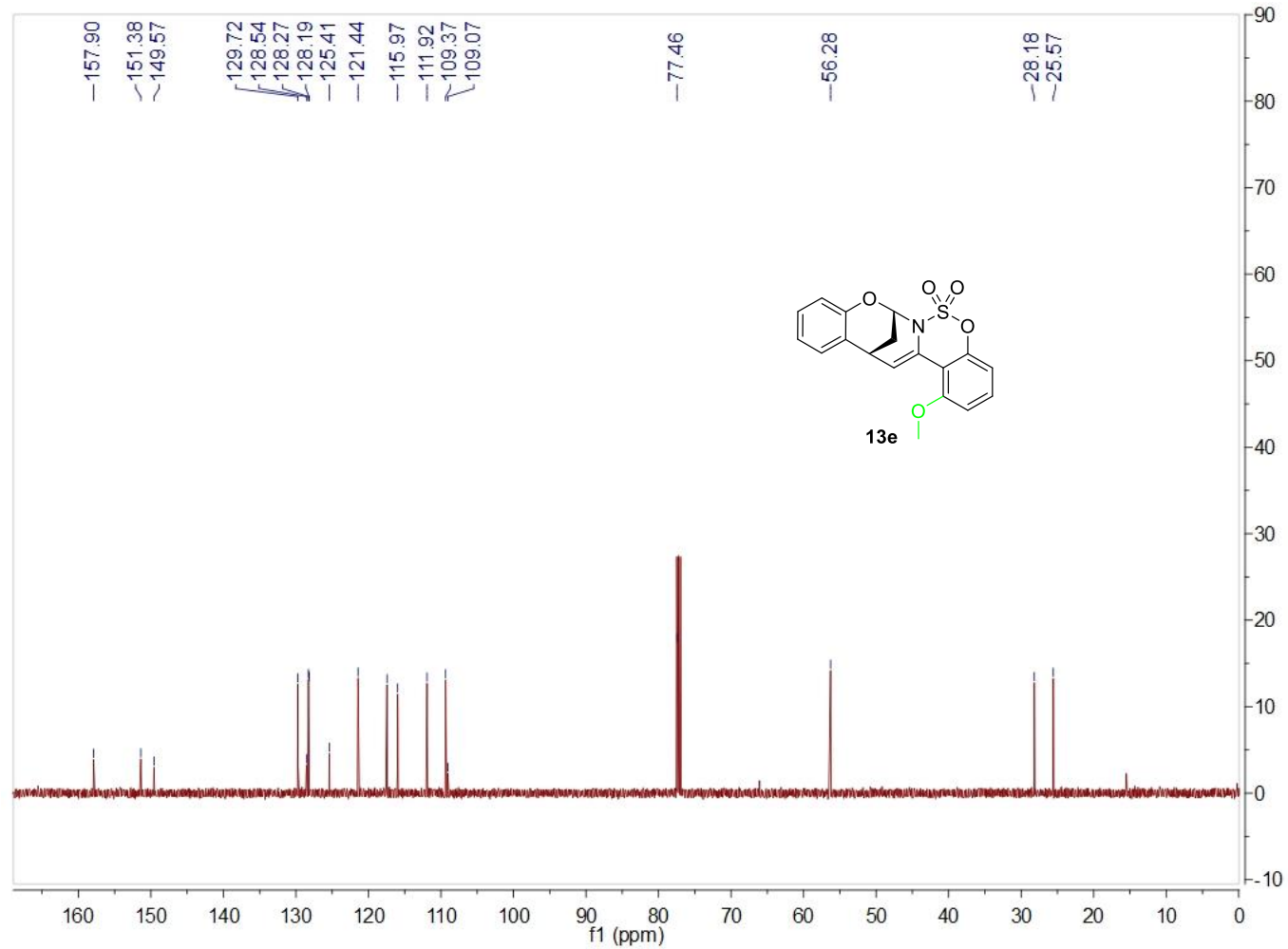

Chiral HPLC analysis of rac-13e

Chrom Type: Fixed WL Chromatogram, $210 \mathrm{~nm}$

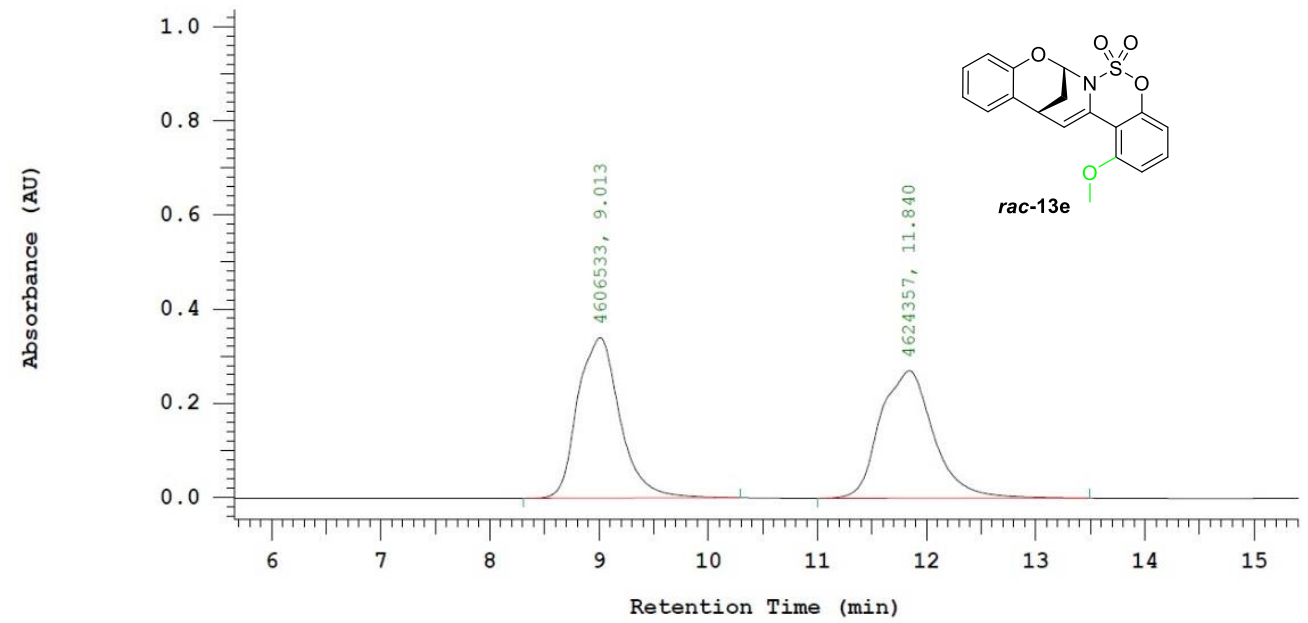

Chrom Type: Fixed WL Chromatogram, $210 \mathrm{~nm}$

Peak Quantitation: AREA

Calculation Method: AREA응

\begin{tabular}{rrrr}
\multicolumn{1}{c}{ RT } & Area & Area & BC \\
\hline 1 & 9.013 & 4606533 & 49.903 \\
2 & 11.840 & 4624357 & 50.097 \\
\hline
\end{tabular}




\section{Chiral HPLC analysis of 13e}

Chrom Type: Fixed WL Chromatogram, 210 nm

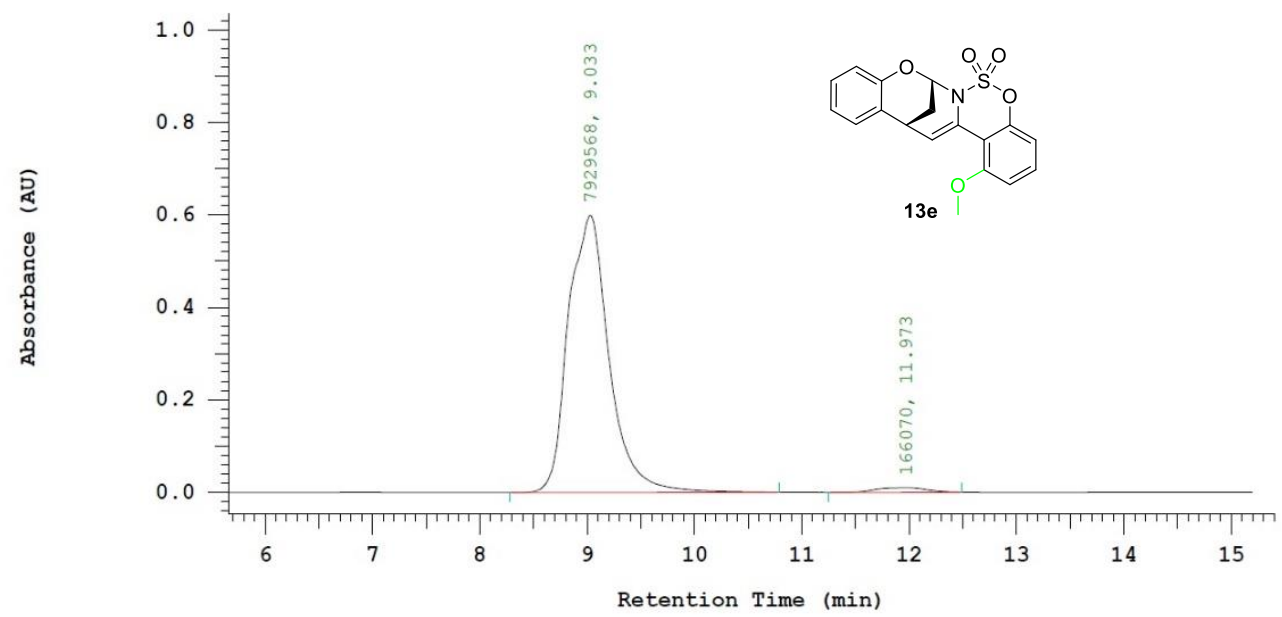

Chrom Type: Fixed WL Chromatogram, $210 \mathrm{~nm}$

Peak Quantitation: AREA

Calculation Method: AREA용

\begin{tabular}{|c|c|c|c|c|}
\hline No. & RT & Area & Area 용 & $\mathrm{BC}$ \\
\hline 1 & 9.033 & 7929568 & 97.949 & BB \\
\hline 2 & 11.973 & 166070 & 2.051 & $\mathrm{BB}$ \\
\hline & & 8095638 & 100.000 & \\
\hline
\end{tabular}

\section{${ }^{1} \mathrm{H}$ NMR of $13 f, \mathrm{CDCl}_{3}$}

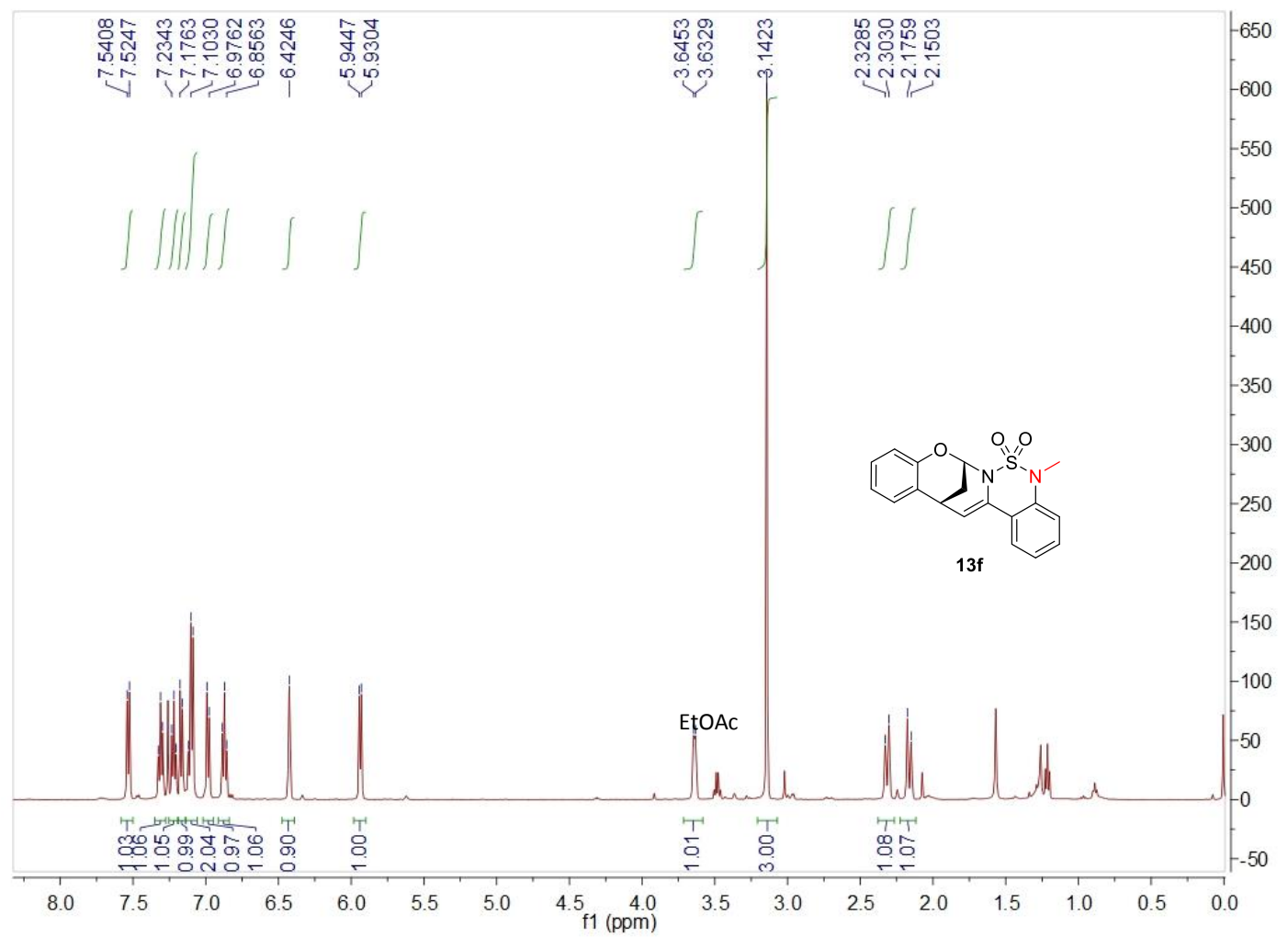


${ }^{13} \mathrm{C} \mathrm{NMR}$ of $13 \mathrm{f}, \mathrm{CDCl}_{3}$

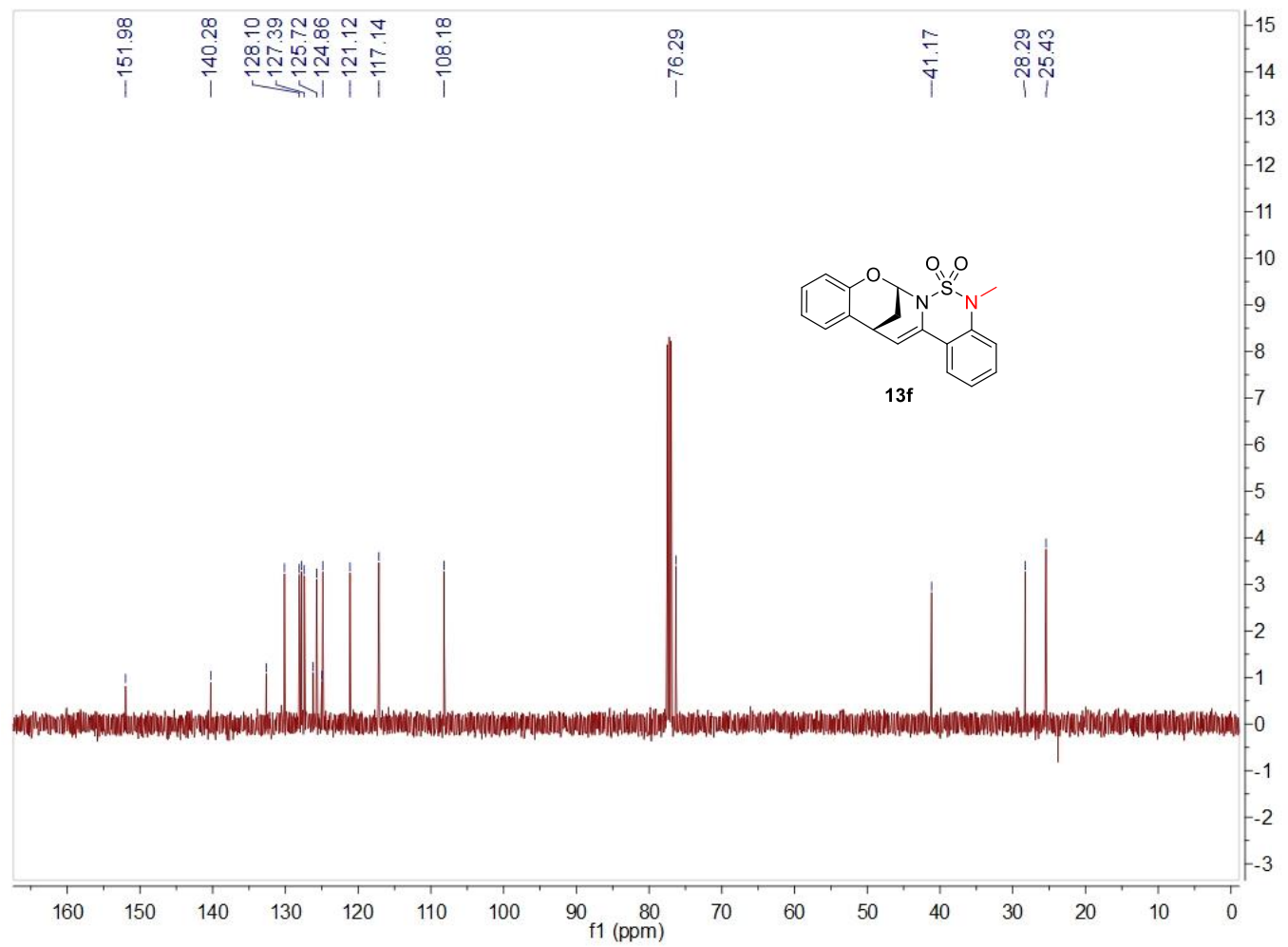

Chiral HPLC analysis of rac-13f

Chrom Type: Fixed WL Chromatogram, $210 \mathrm{~nm}$

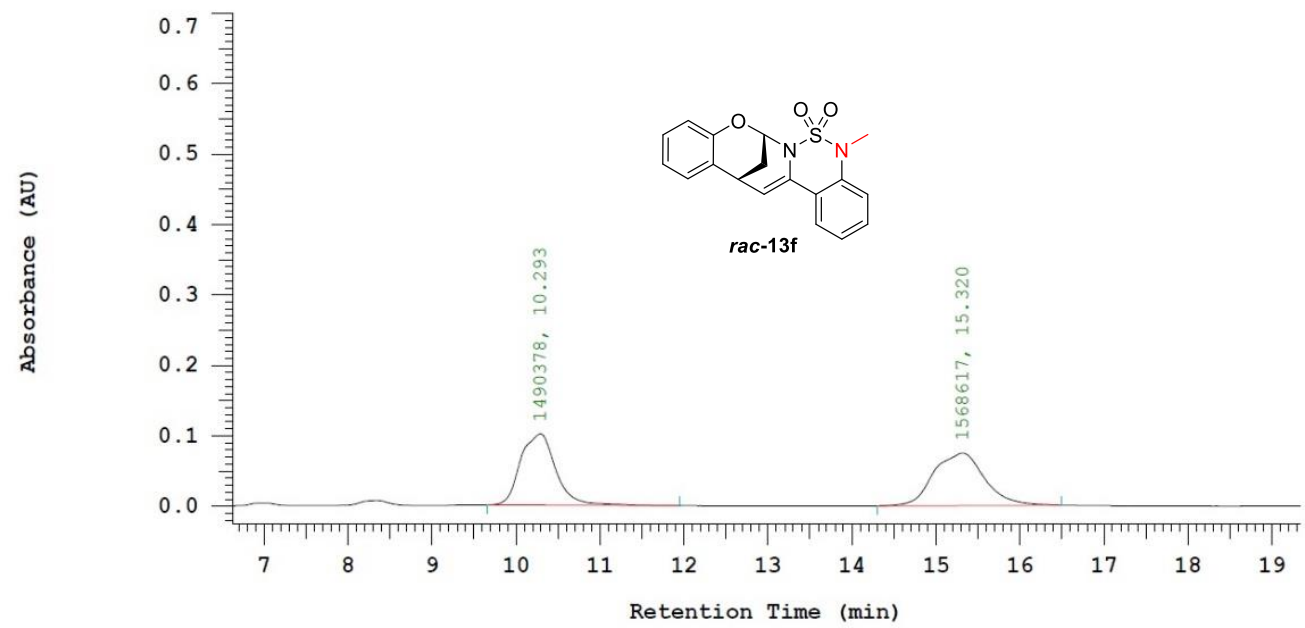

Chrom Type: Fixed WL Chromatogram, $210 \mathrm{~nm}$

Peak Quantitation: AREA

Calculation Method: AREA응

\begin{tabular}{rrrrr} 
No. & RT & Area & Area 은 & BC \\
\hline 1 & 10.293 & 1490378 & 48.721 & BB \\
2 & 15.320 & 1568617 & 51.279 & BB \\
\hline & & 3058995 & 100.000 & \\
\hline
\end{tabular}




\section{Chiral HPLC analysis of $\mathbf{1 3 f}$}

Chrom Type: Fixed WL Chromatogram, $210 \mathrm{~nm}$

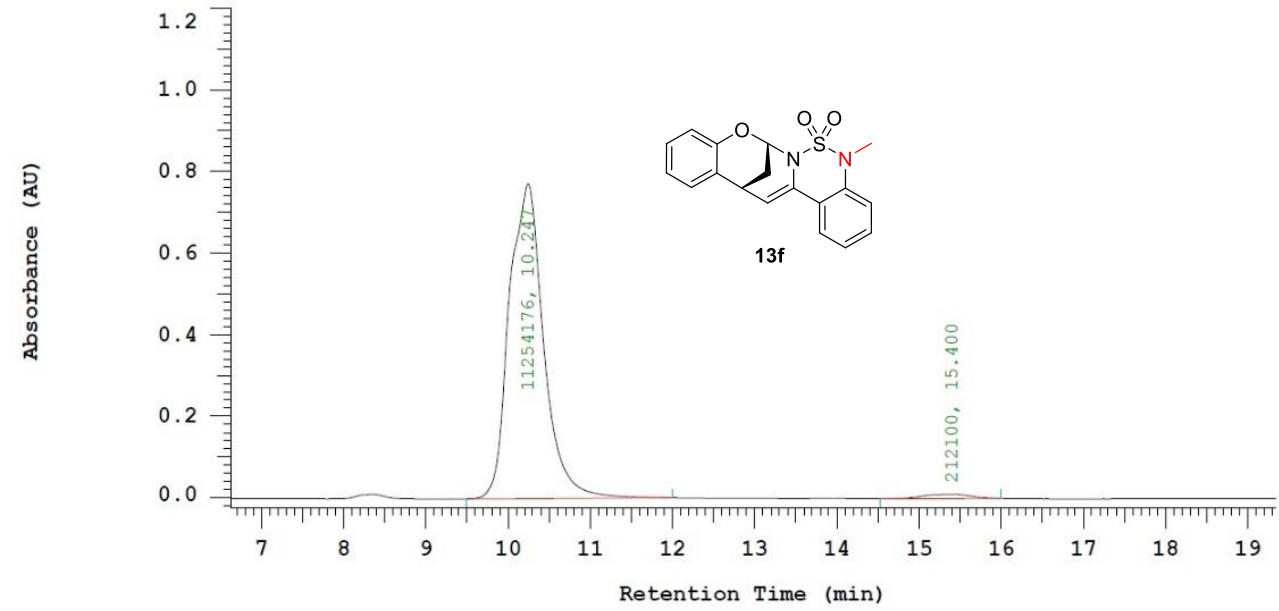

Chrom Type: Fixed WL Chromatogram, $210 \mathrm{~nm}$

Peak Quantitation: AREA

Calculation Method: AREA응

\begin{tabular}{|c|c|c|c|c|}
\hline No. & $\mathrm{RT}$ & Area & Area $\frac{\circ}{\partial}$ & $\mathrm{BC}$ \\
\hline 1 & 10.247 & 11254176 & 98.150 & $\mathrm{BB}$ \\
\hline \multirow[t]{2}{*}{2} & 15.400 & 212100 & 1.850 & $\mathrm{BB}$ \\
\hline & & 11466276 & 100.000 & \\
\hline
\end{tabular}

${ }^{1} \mathrm{H} \mathrm{NMR} \mathrm{of} 7 \mathrm{a}, \mathrm{CDCl}_{3}$

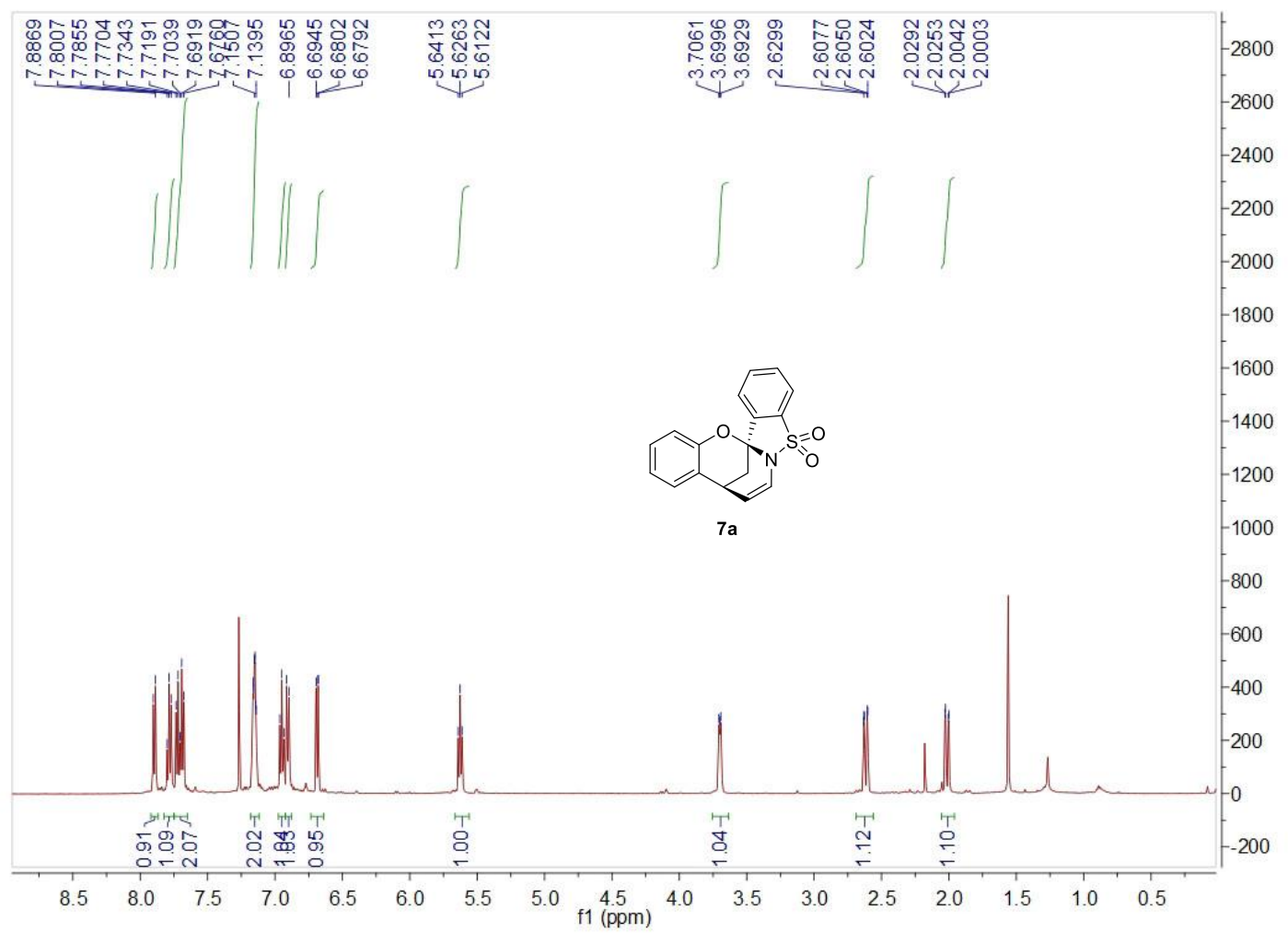


${ }^{13} \mathrm{C}$ NMR of $7 \mathrm{a}, \mathrm{CDCl}_{3}$

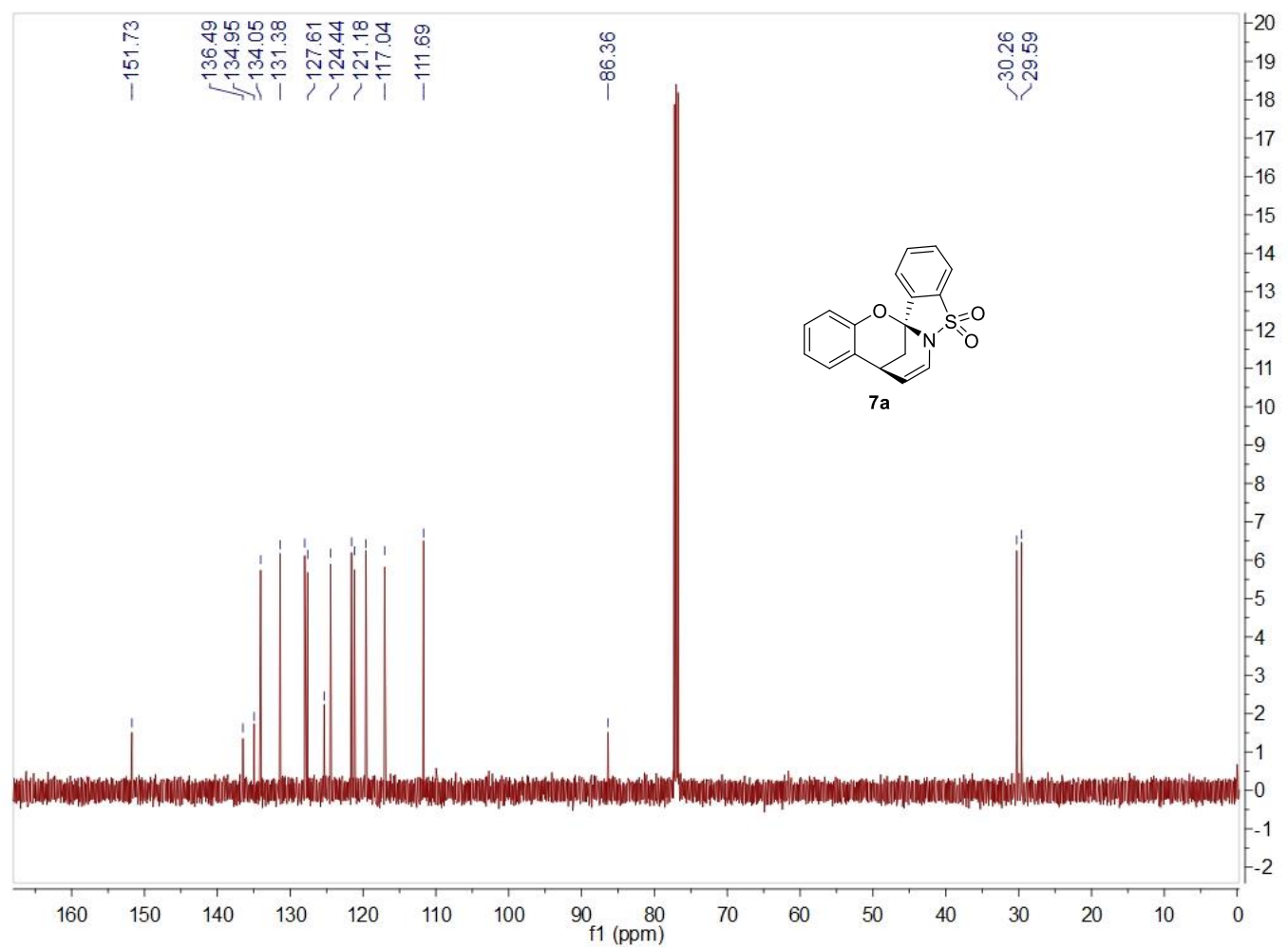

${ }^{1} \mathrm{H}-{ }^{1} \mathrm{H}$ COSY of $7 \mathrm{a}, \mathrm{CDCl}_{3}$

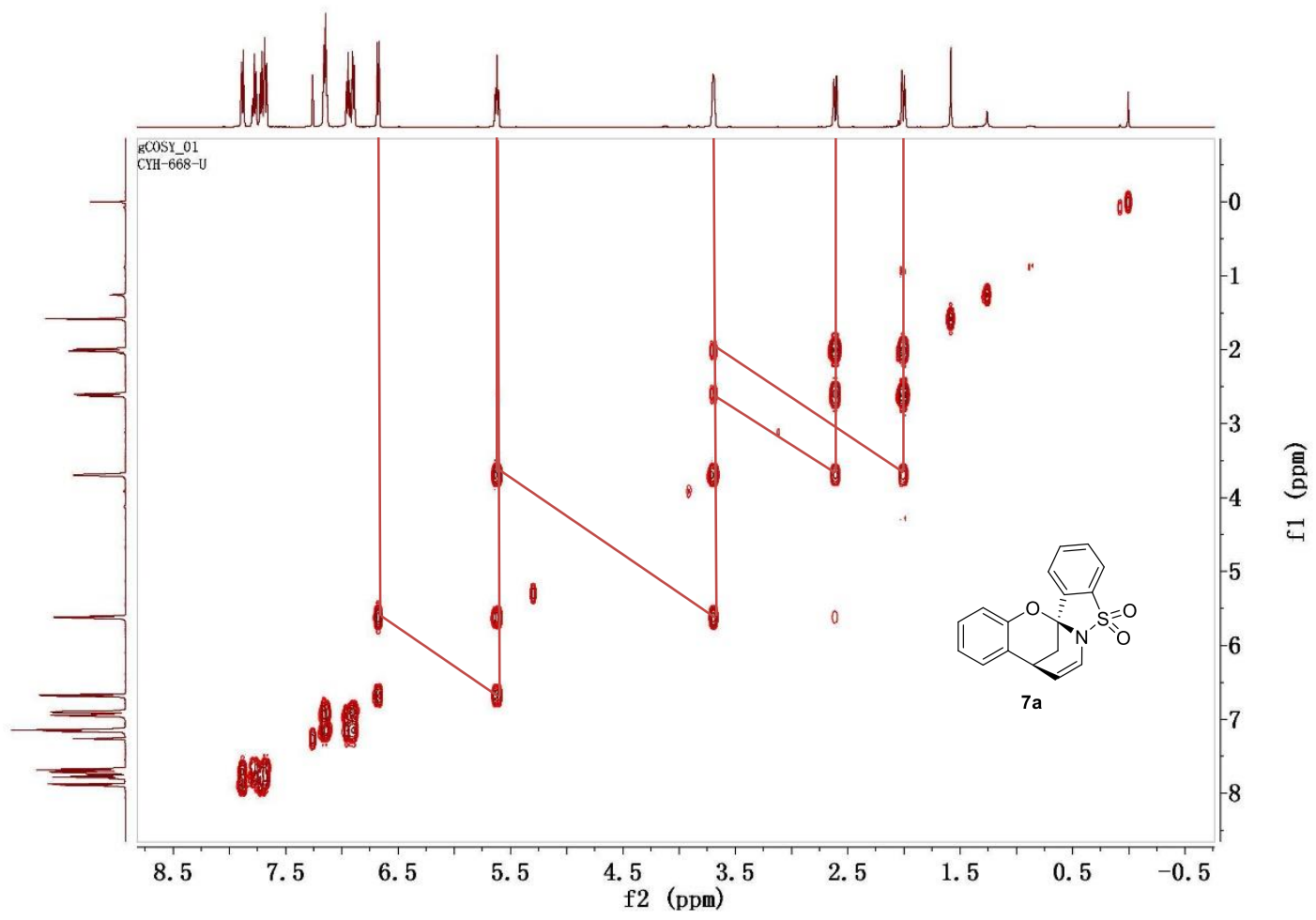


${ }^{1} \mathrm{H}$ NMR of $14 \mathrm{a}^{\prime}, \mathrm{CDCl}_{3}$

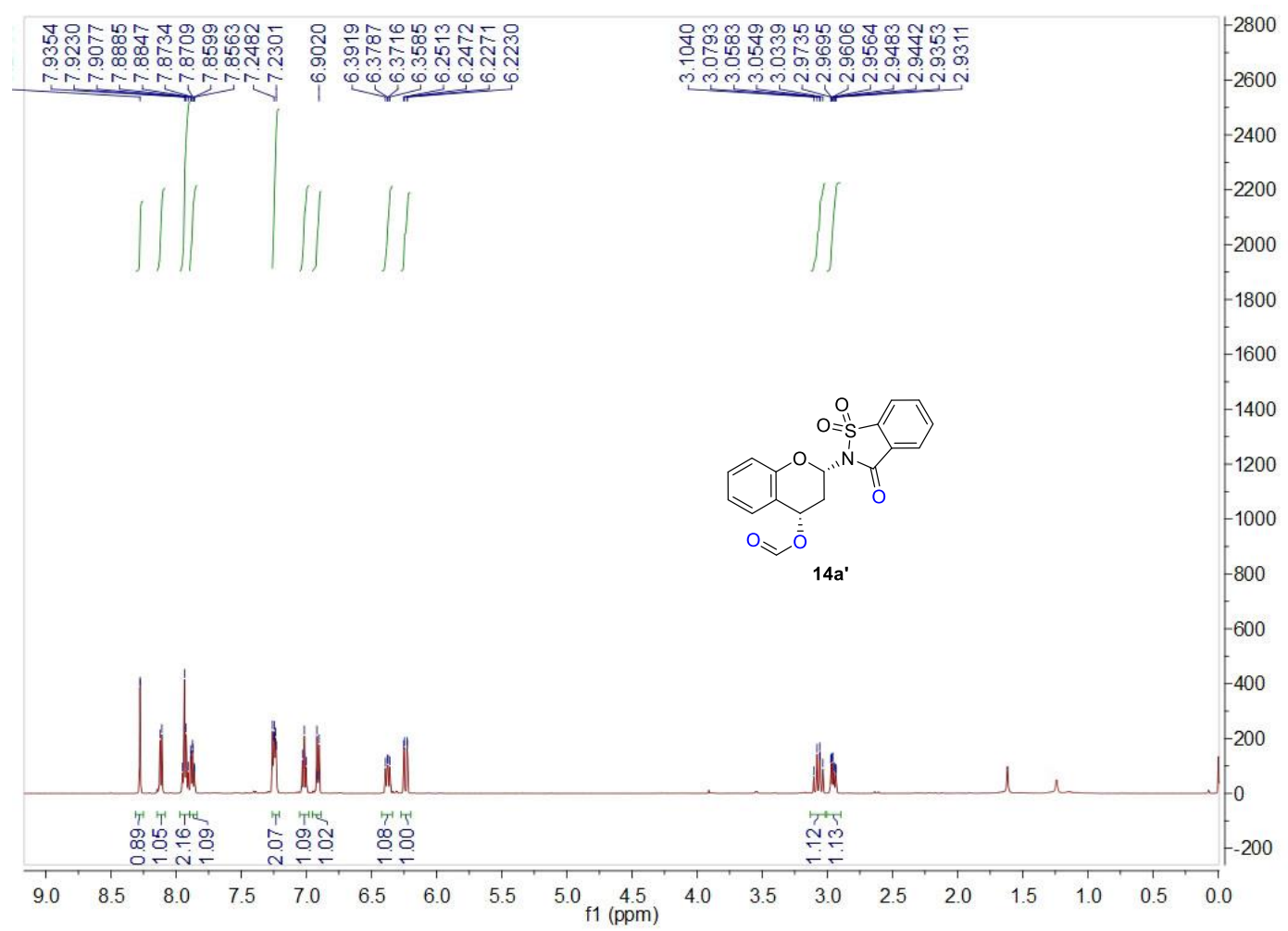

${ }^{13} \mathrm{C}$ NMR of $14 a^{\prime}, \mathrm{CDCl}_{3}$

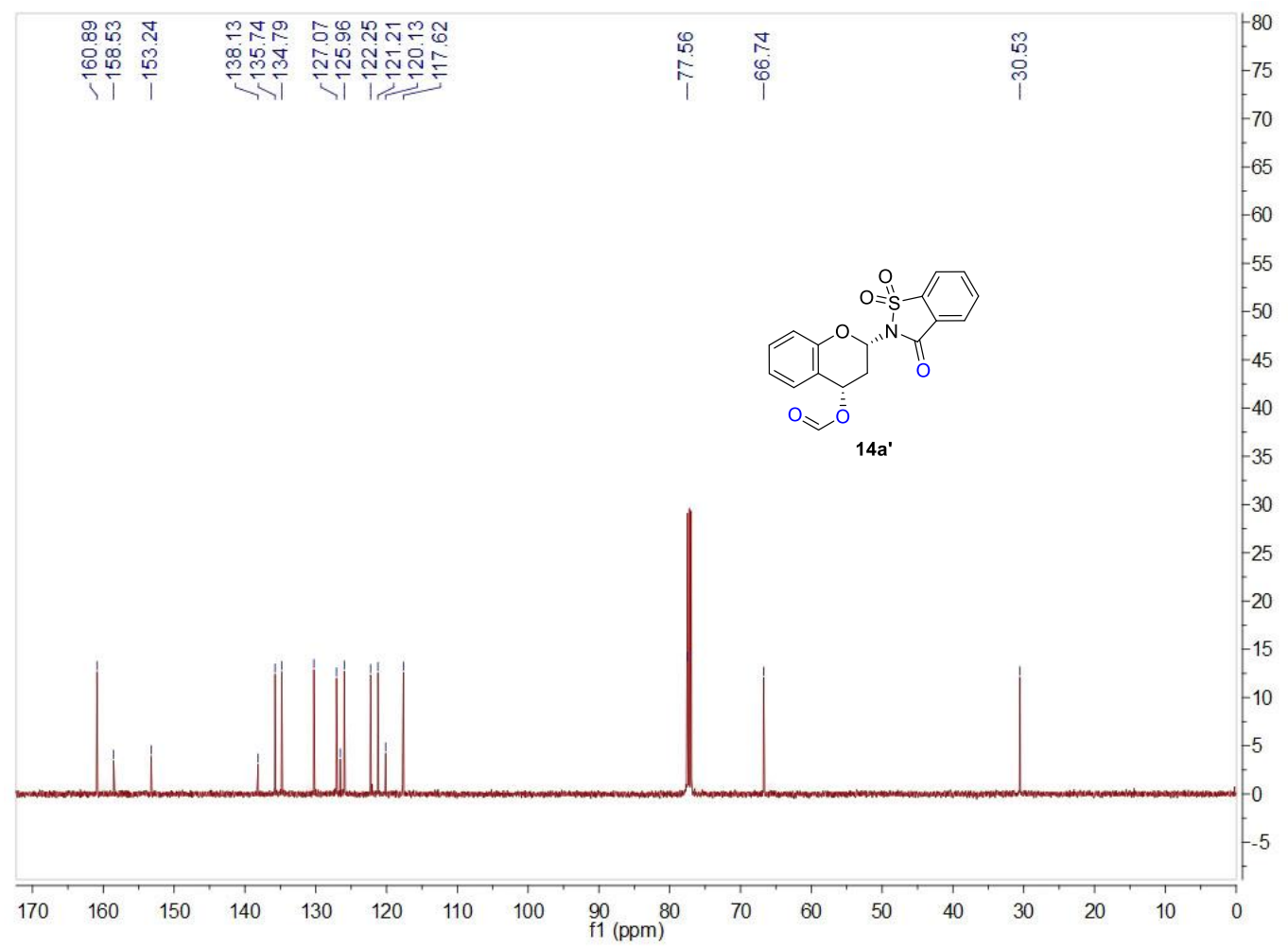




\section{Chiral HPLC analysis of rac-14a'}

Chrom Type: Fixed WL Chromatogram, $216 \mathrm{~nm}$

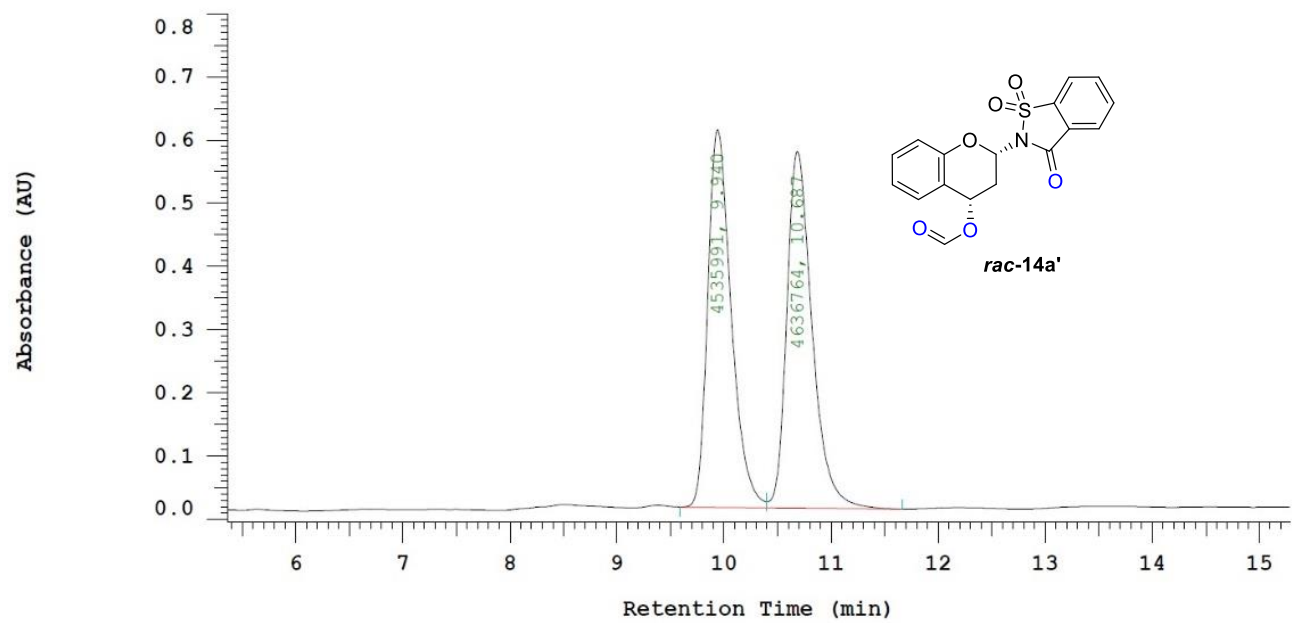

Chrom Type: Fixed WL Chromatogram, $216 \mathrm{~nm}$

Peak Quantitation: AREA

Calculation Method: AREA응

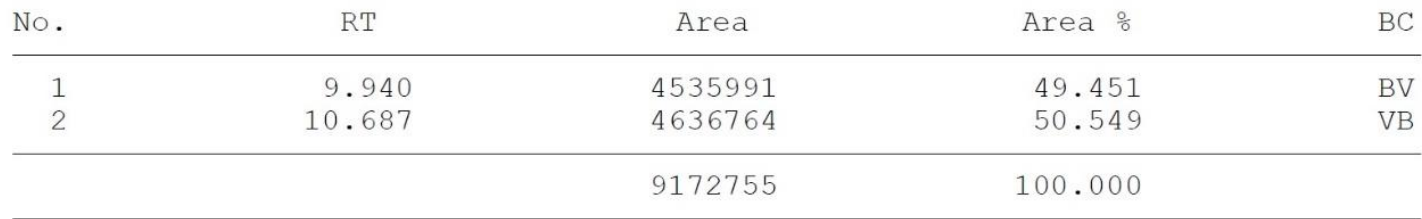

\section{Chiral HPLC analysis of 14a'}

Chrom Type: Fixed WL Chromatogram, 216 nm

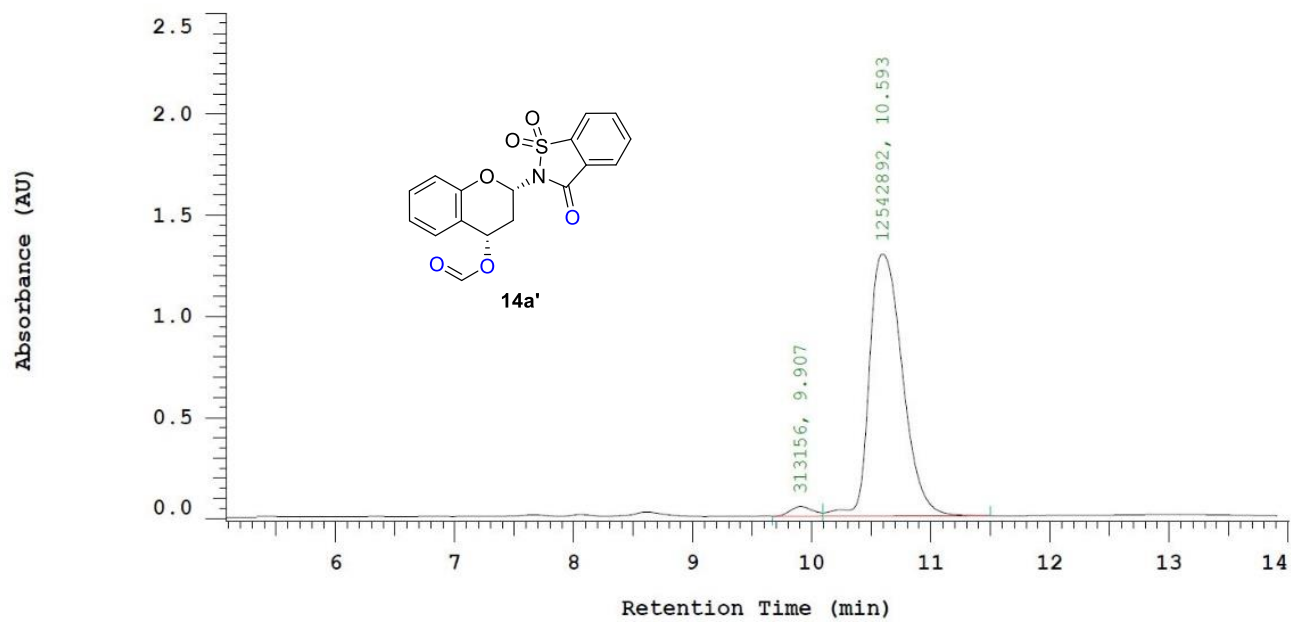

Chrom Type: Fixed WL Chromatogram, $216 \mathrm{~nm}$

Peak Quantitation: AREA

Calculation Method: AREA응

\begin{tabular}{|c|c|c|c|c|}
\hline No. & RT & Area & Area \% & $\mathrm{BC}$ \\
\hline 1 & 9.907 & 313156 & 2.436 & BV \\
\hline 2 & 10.593 & 12542892 & 97.564 & VB \\
\hline & & 12856048 & 100.000 & \\
\hline
\end{tabular}


${ }^{1} \mathrm{H}$ NMR of $14 \mathrm{a}, \mathrm{CDCl}_{3}$

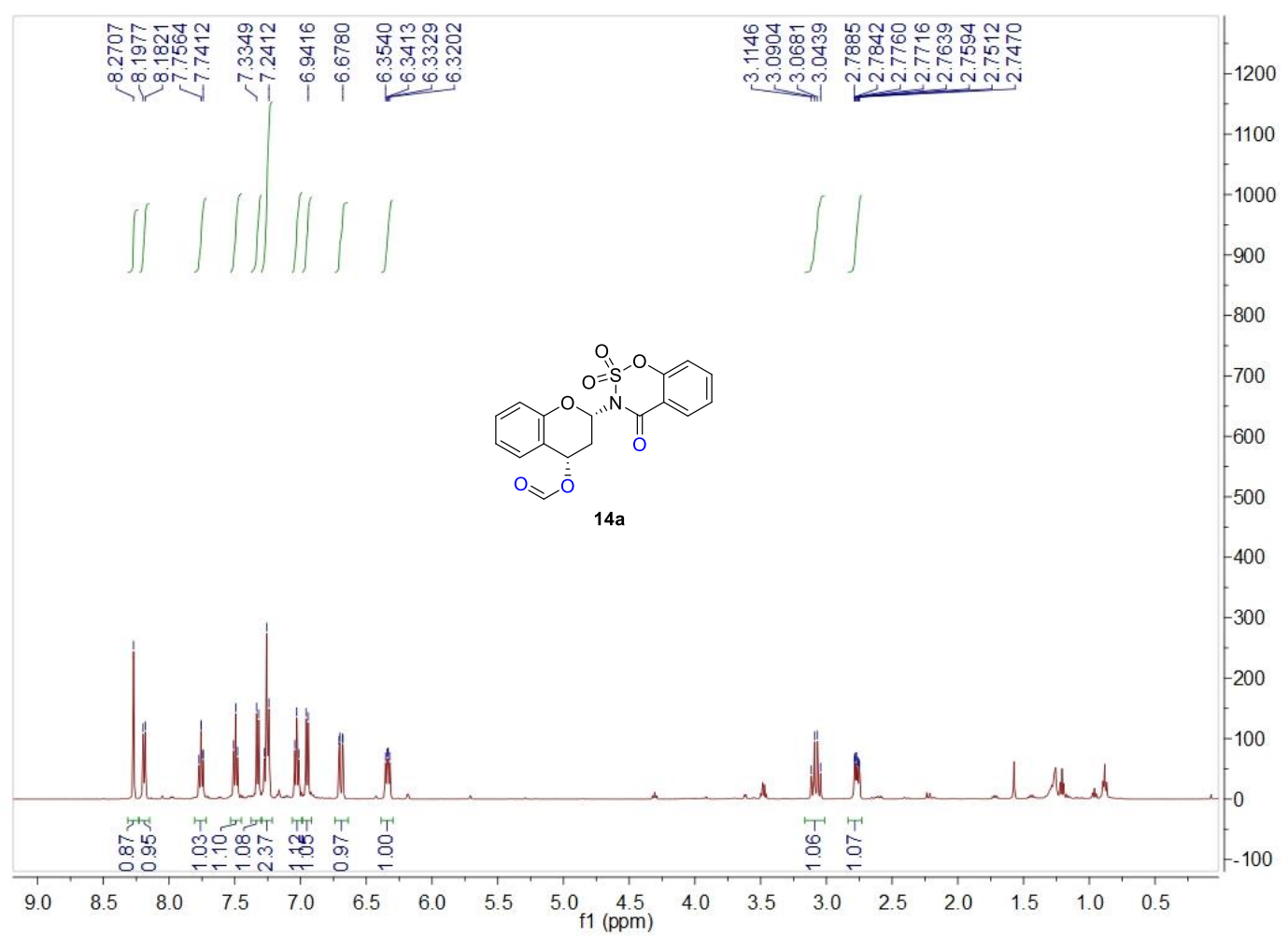

${ }^{13} \mathrm{C}$ NMR of $14 a, \mathrm{CDCl}_{3}$

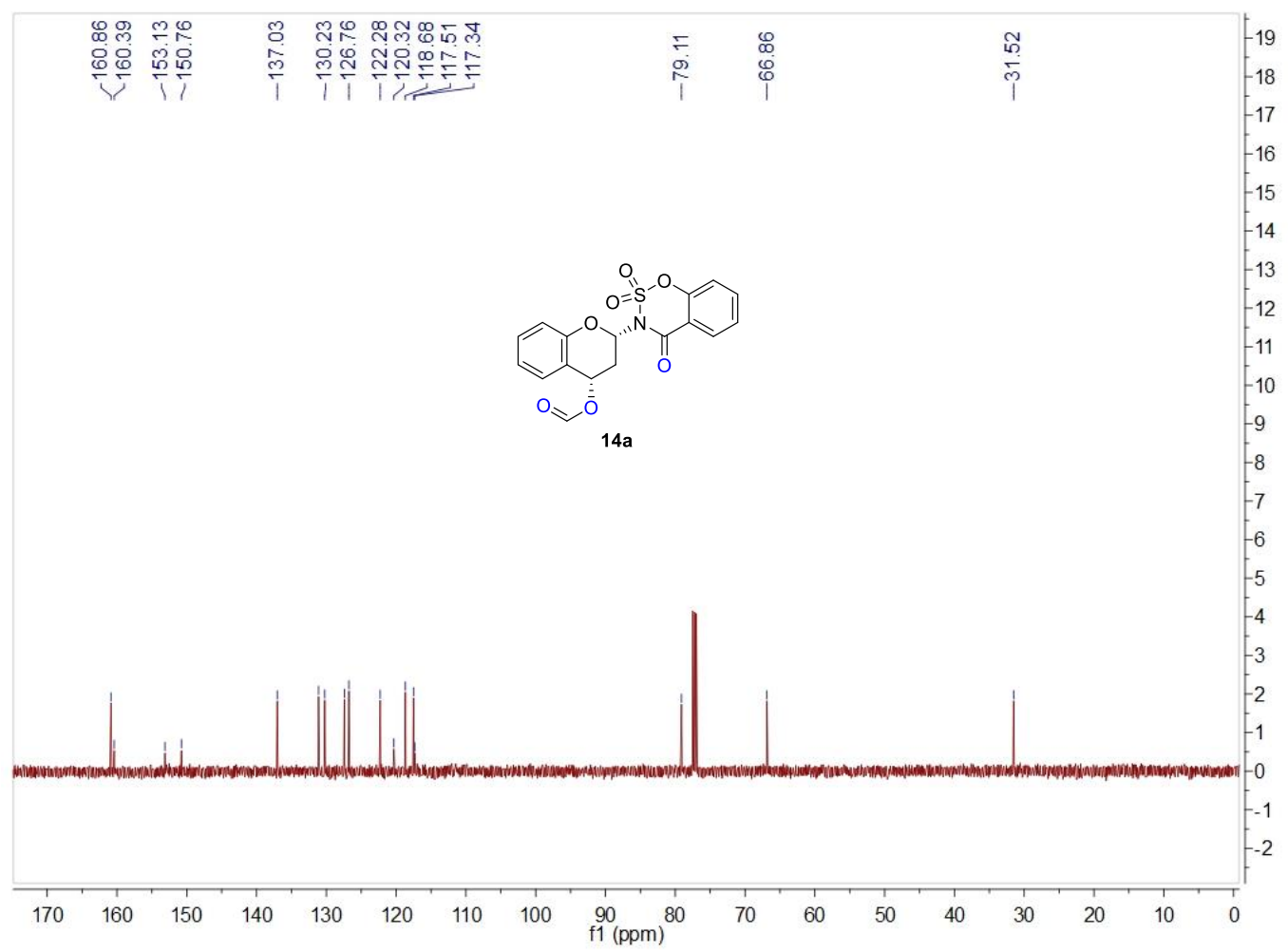




\section{Chiral HPLC analysis of rac-14a}

Chrom Type: Fixed WL Chromatogram, $210 \mathrm{~nm}$

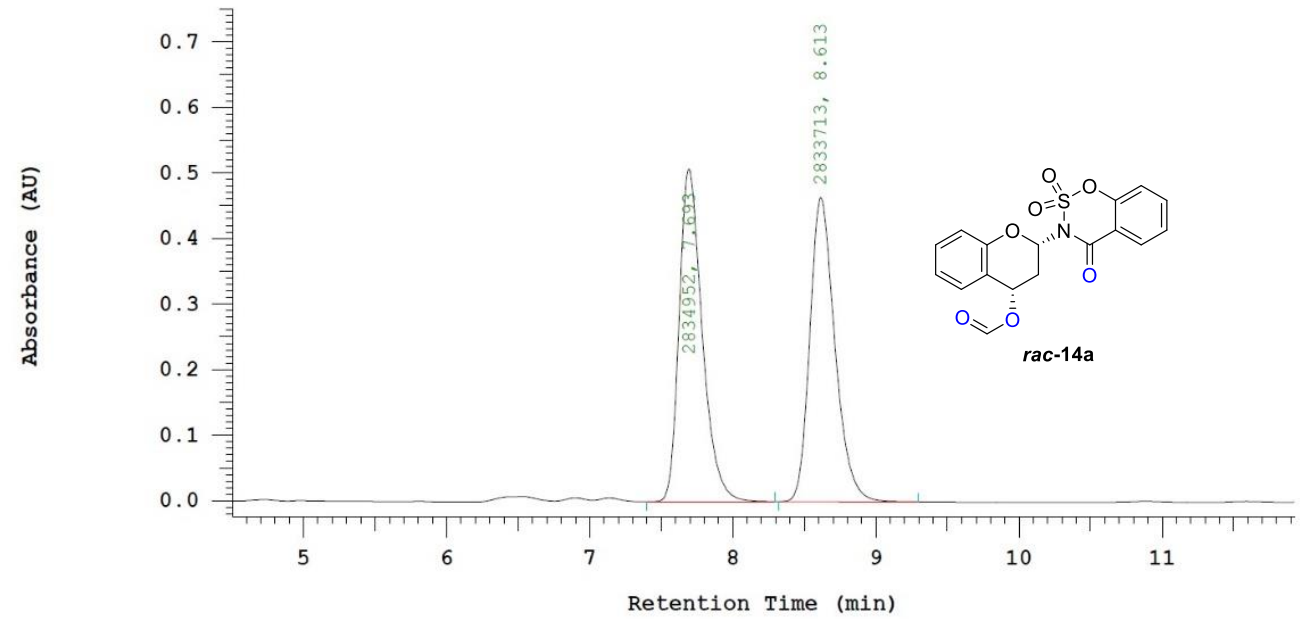

Chrom Type: Fixed WL Chromatogram, $210 \mathrm{~nm}$

Peak Quantitation: AREA

Calculation Method: AREA응

\begin{tabular}{ccccc} 
No. & RT & Area & Area & BC \\
\hline 1 & 7.693 & 2834952 & 50.011 & BB \\
2 & 8.613 & 2833713 & 49.989 & BB \\
\hline & & 5668665 & 100.000
\end{tabular}

\section{Chiral HPLC analysis of $14 a$}

Chrom Type: Fixed WL Chromatogram, $210 \mathrm{~nm}$

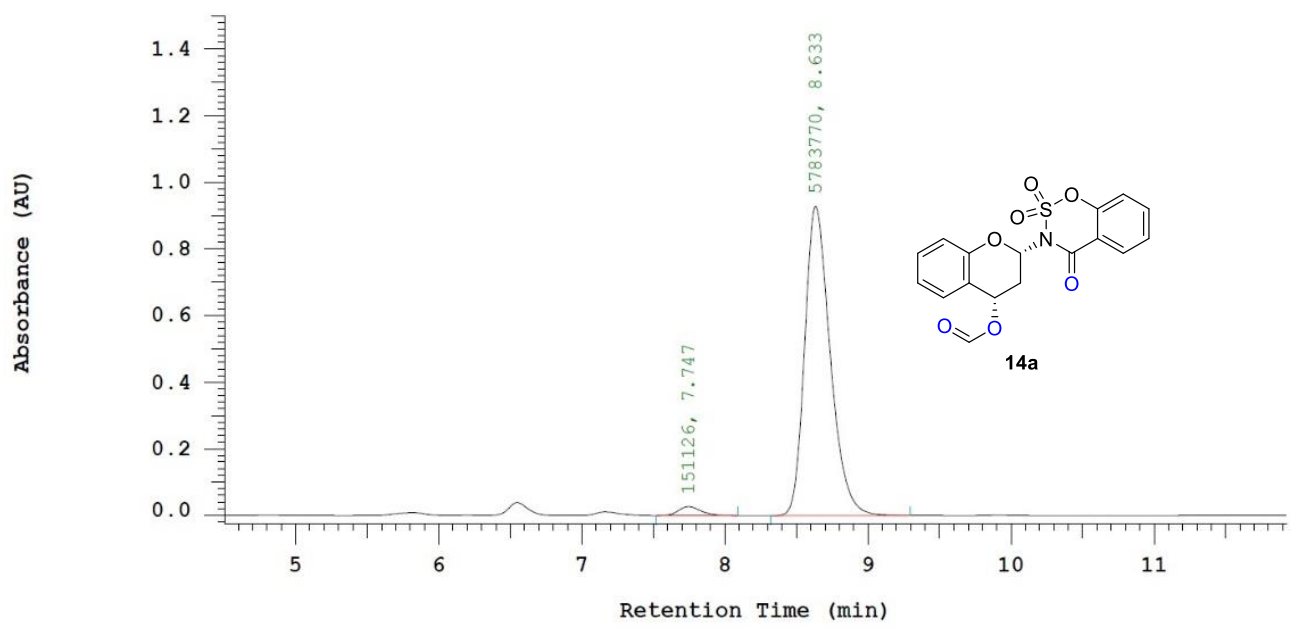

Chrom Type: Fixed WL Chromatogram, $210 \mathrm{~nm}$

Peak Quantitation: AREA

Calculation Method: AREA\%

\begin{tabular}{|c|c|c|c|c|}
\hline No. & $\mathrm{RT}$ & Area & Area 응 & $\mathrm{BC}$ \\
\hline 1 & 7.747 & 151126 & 2.546 & $\mathrm{BB}$ \\
\hline \multirow[t]{2}{*}{2} & 8.633 & 5783770 & 97.454 & $\mathrm{BB}$ \\
\hline & & 5934896 & 100.000 & \\
\hline
\end{tabular}


${ }^{1} \mathrm{H}$ NMR of $14 \mathrm{c}, \mathrm{CDCl}_{3}$

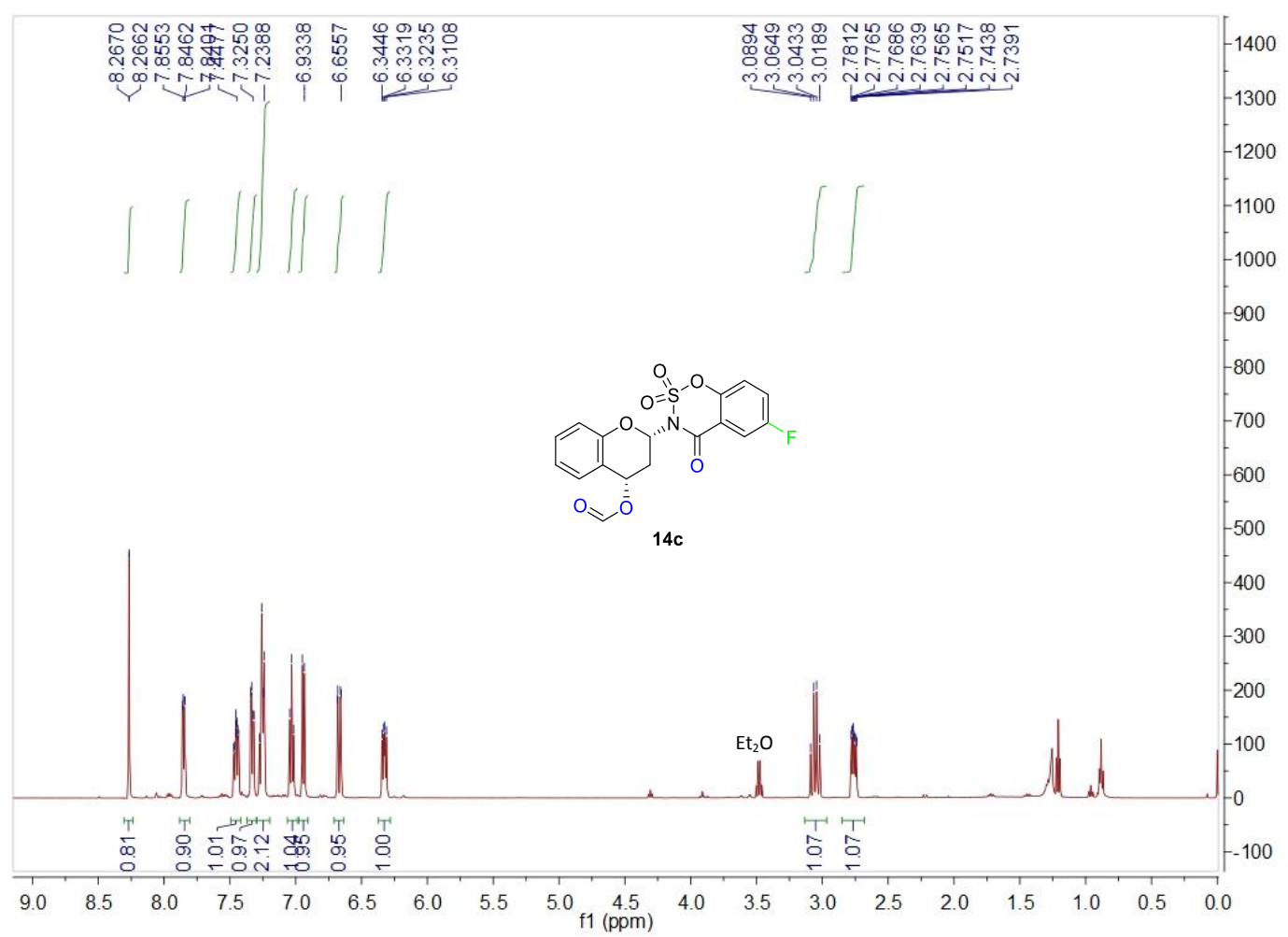

${ }^{13} \mathrm{CNMR}$ of $14 \mathrm{c}, \mathrm{CDCl}_{3}$

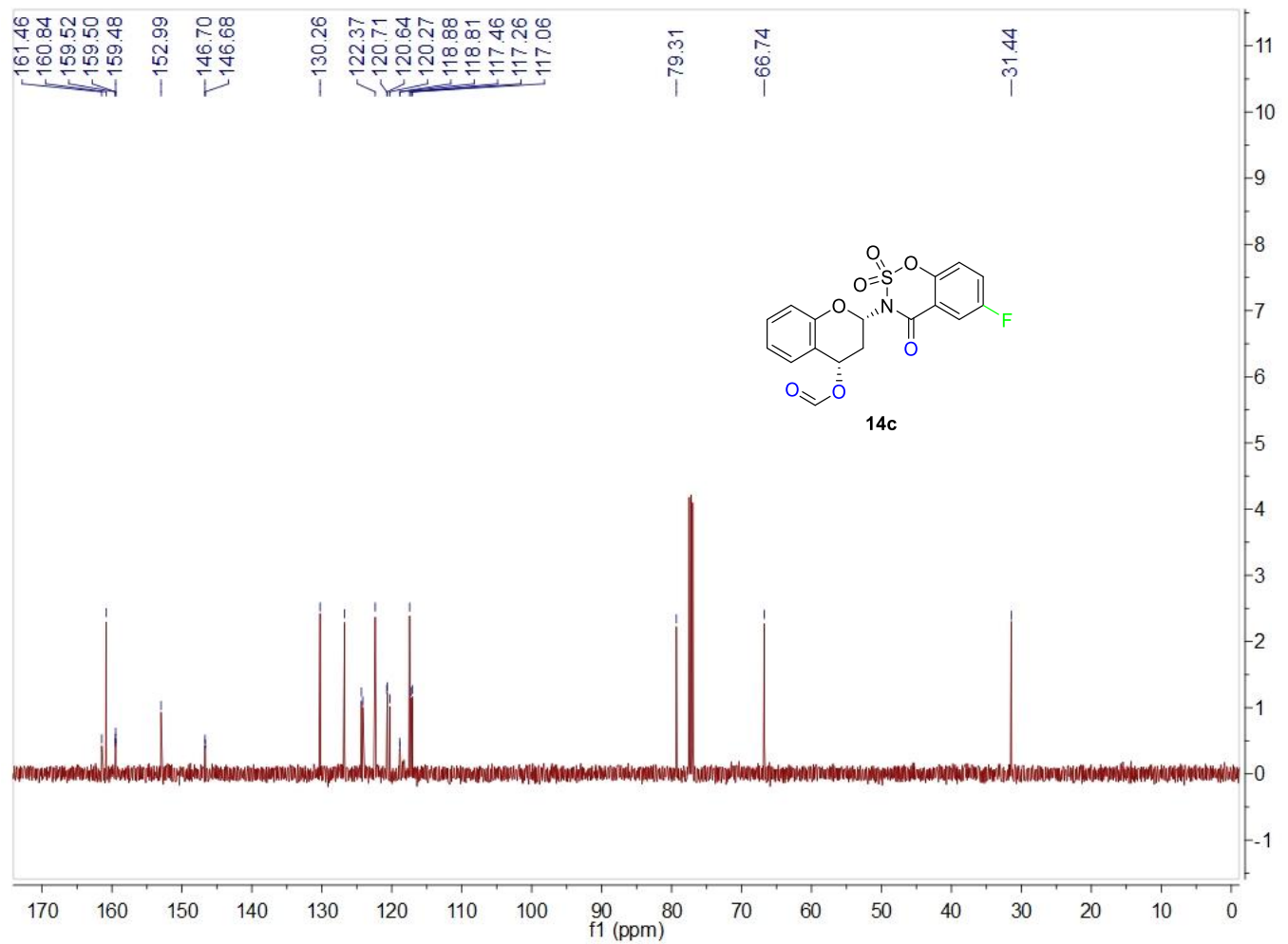




\section{Chiral HPLC analysis of rac-14c}

Chrom Type: Fixed WL Chromatogram, $210 \mathrm{~nm}$

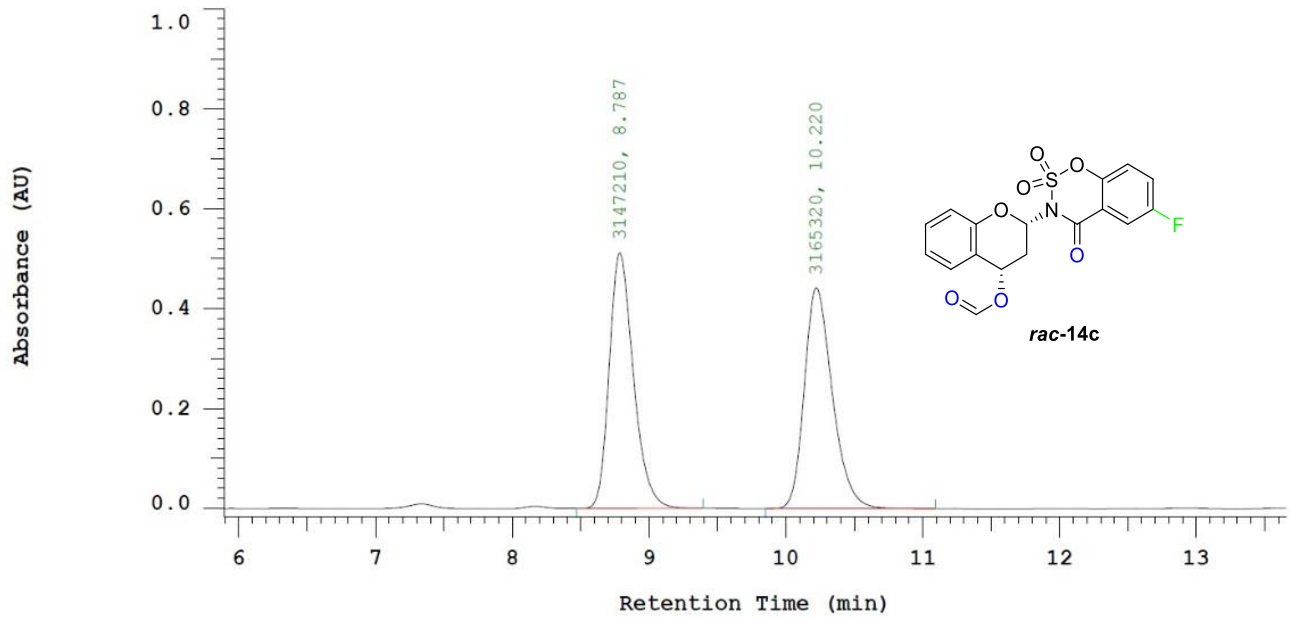

Chrom Type: Fixed WL Chromatogram, $210 \mathrm{~nm}$

Peak Quantitation: AREA

Calculation Method: AREA\%

\begin{tabular}{|c|c|c|c|c|}
\hline No. & $\mathrm{RT}$ & Area & Area 응 & $\mathrm{BC}$ \\
\hline 1 & 8.787 & 3147210 & 49.857 & $\mathrm{BB}$ \\
\hline \multirow[t]{2}{*}{2} & 10.220 & 3165320 & 50.143 & $\mathrm{BB}$ \\
\hline & & 6312530 & 100.000 & \\
\hline
\end{tabular}

\section{Chiral HPLC analysis of 14c}

Chrom Type: Fixed WL Chromatogram, $210 \mathrm{~nm}$

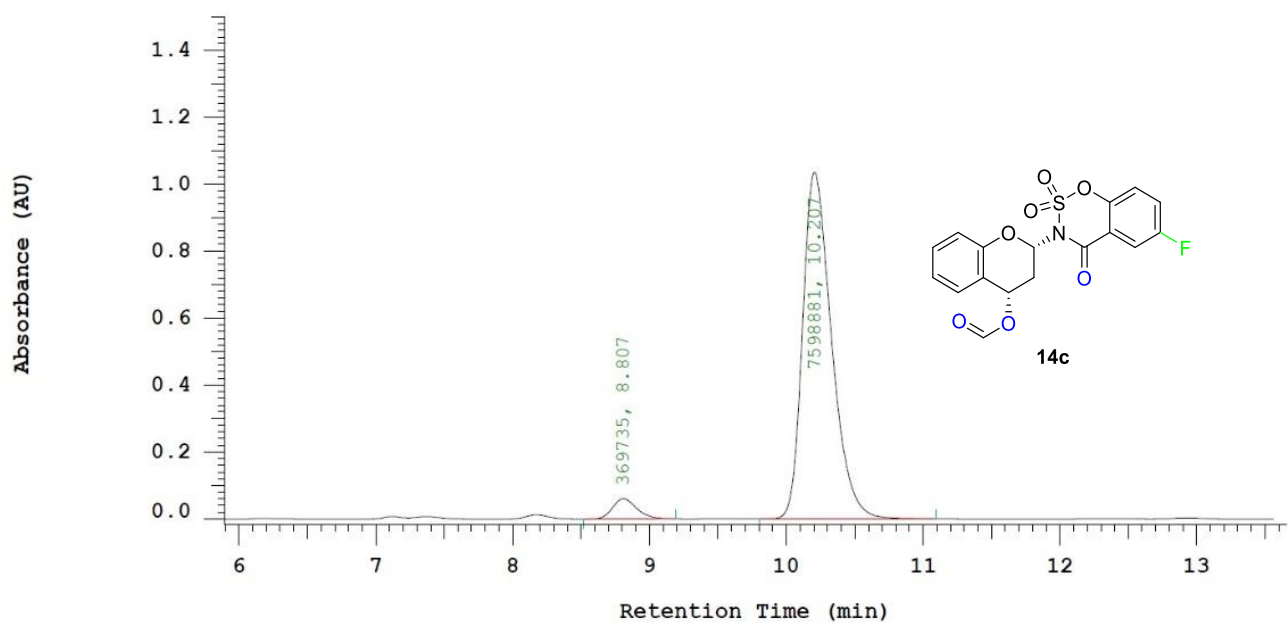

Chrom Type: Fixed WL Chromatogram, $210 \mathrm{~nm}$

Peak Quantitation: AREA

Calculation Method: AREA응

\begin{tabular}{|c|c|c|c|c|}
\hline No. & $\mathrm{RT}$ & Area & Area 응 & $\mathrm{BC}$ \\
\hline 1 & 8.807 & 369735 & 4.640 & BB \\
\hline \multirow[t]{2}{*}{2} & 10.207 & 7598881 & 95.360 & BB \\
\hline & & 7968616 & 100.000 & \\
\hline
\end{tabular}


${ }^{1} \mathrm{H}$ NMR of $14 \mathrm{~d}, \mathrm{CDCl}_{3}$

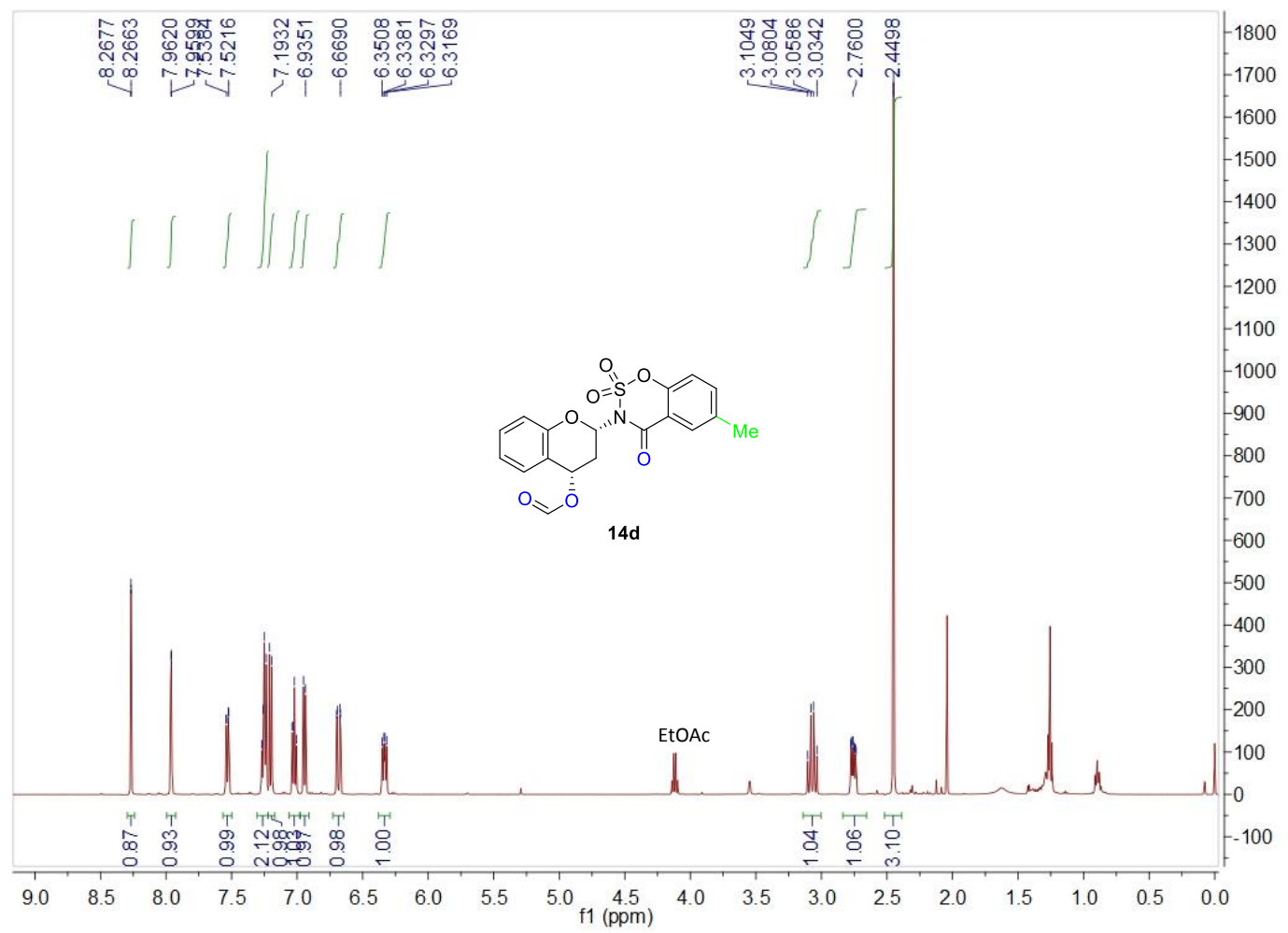

${ }^{13} \mathrm{CNMR}$ of $14 \mathrm{~d}, \mathrm{CDCl}_{3}$

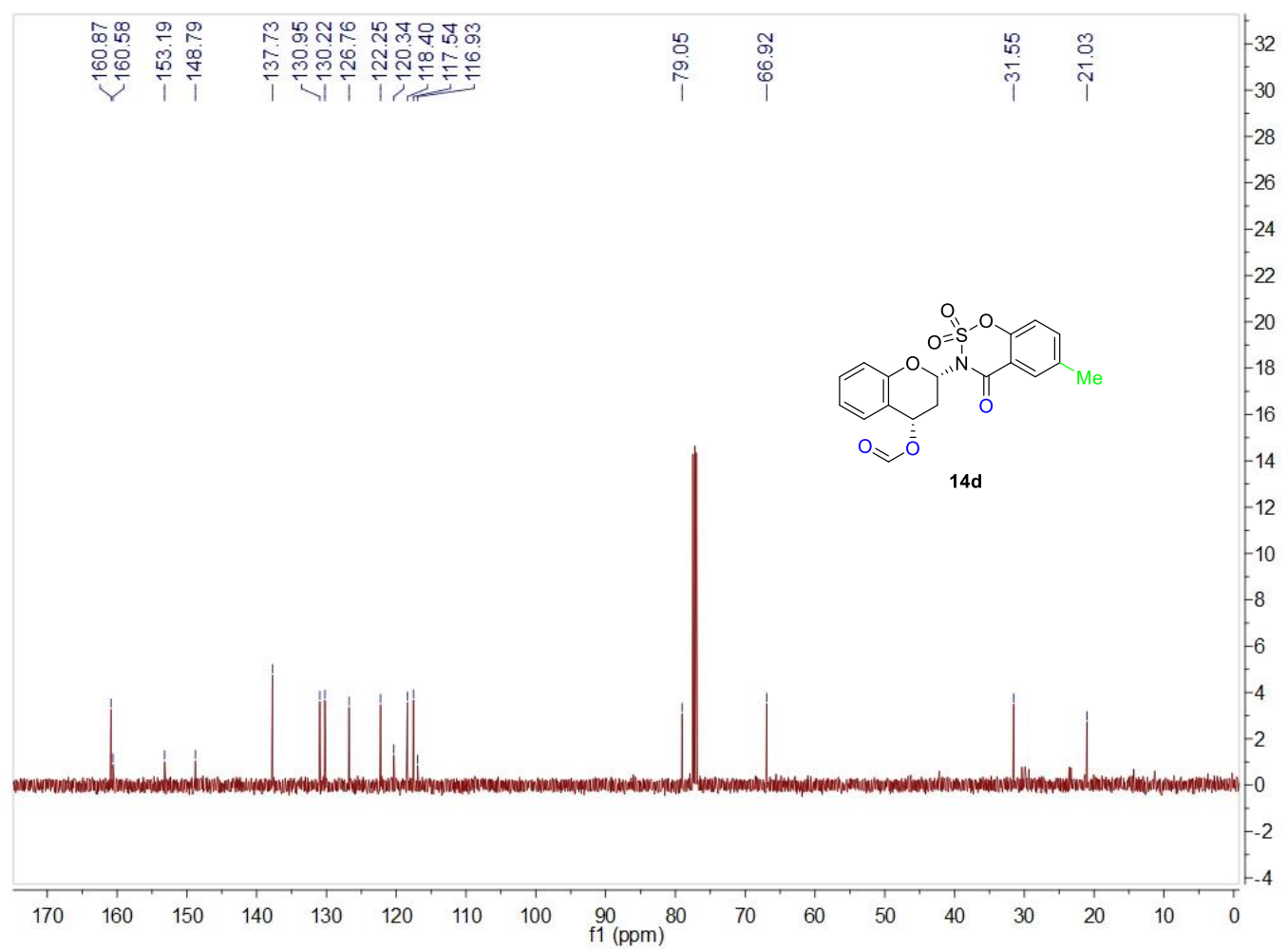




\section{Chiral HPLC analysis of rac-14d}

Chrom Type: Fixed WL Chromatogram, 210 nm

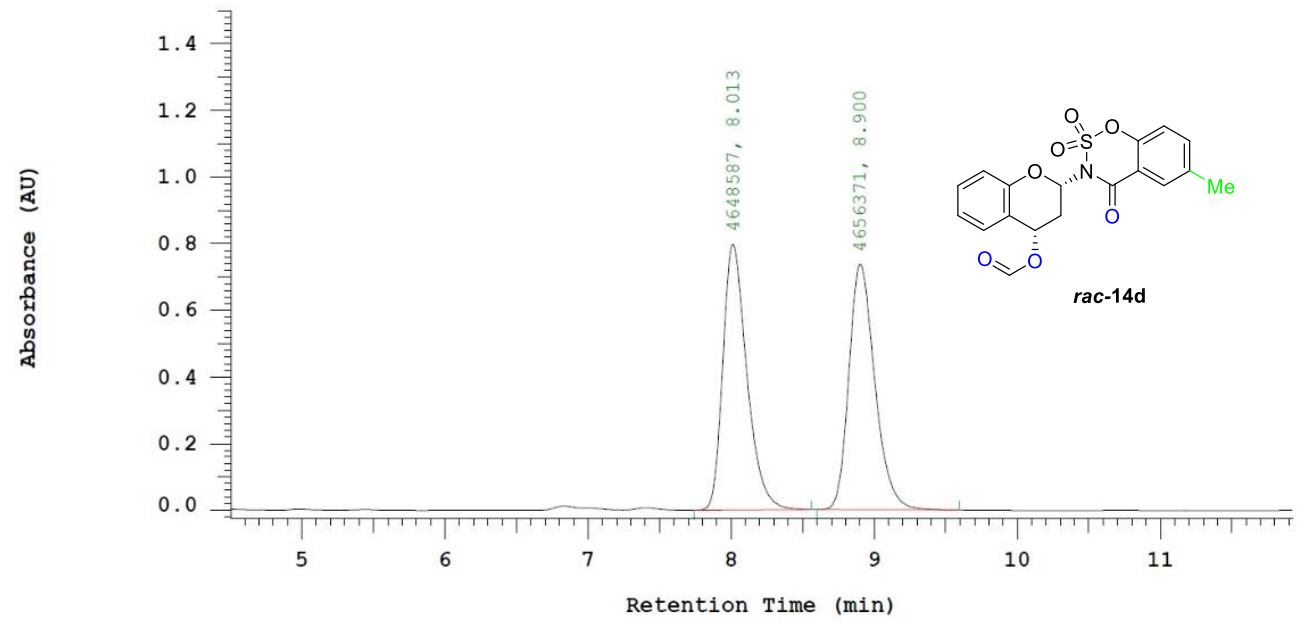

Chrom Type: Fixed WL Chromatogram, $210 \mathrm{~nm}$

Peak Quantitation: AREA

Calculation Method: AREA응

\begin{tabular}{ccccc} 
No. & RT & Area & Area & BC \\
\hline 1 & 8.013 & 4648587 & 49.958 & BB \\
2 & 8.900 & 4656371 & 50.042 & BB \\
\hline & & 9304958 & 100.000 \\
\hline
\end{tabular}

\section{Chiral HPLC analysis of 14d}

Chrom Type: Fixed WL Chromatogram, $210 \mathrm{~nm}$

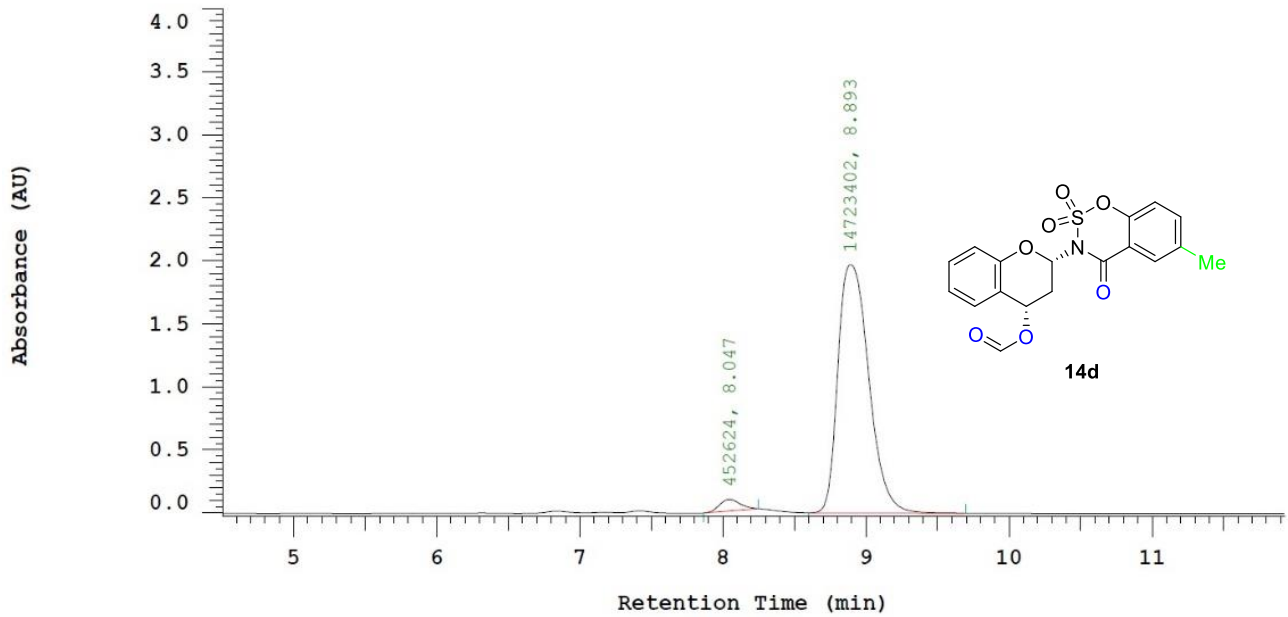

Chrom Type: Fixed WL Chromatogram, $210 \mathrm{~nm}$

Peak Quantitation: AREA

Calculation Method: AREA응

\begin{tabular}{|c|c|c|c|c|}
\hline No. & $\mathrm{RT}$ & Area & Area \% & $\mathrm{BC}$ \\
\hline 1 & 8.047 & 452624 & 2.982 & $\mathrm{BB}$ \\
\hline \multirow[t]{2}{*}{2} & 8.893 & 14723402 & 97.018 & $\mathrm{BB}$ \\
\hline & & 15176026 & 100.000 & \\
\hline
\end{tabular}


${ }^{1} \mathrm{H}$ NMR of $15 b, \mathrm{CDCl}_{3}$

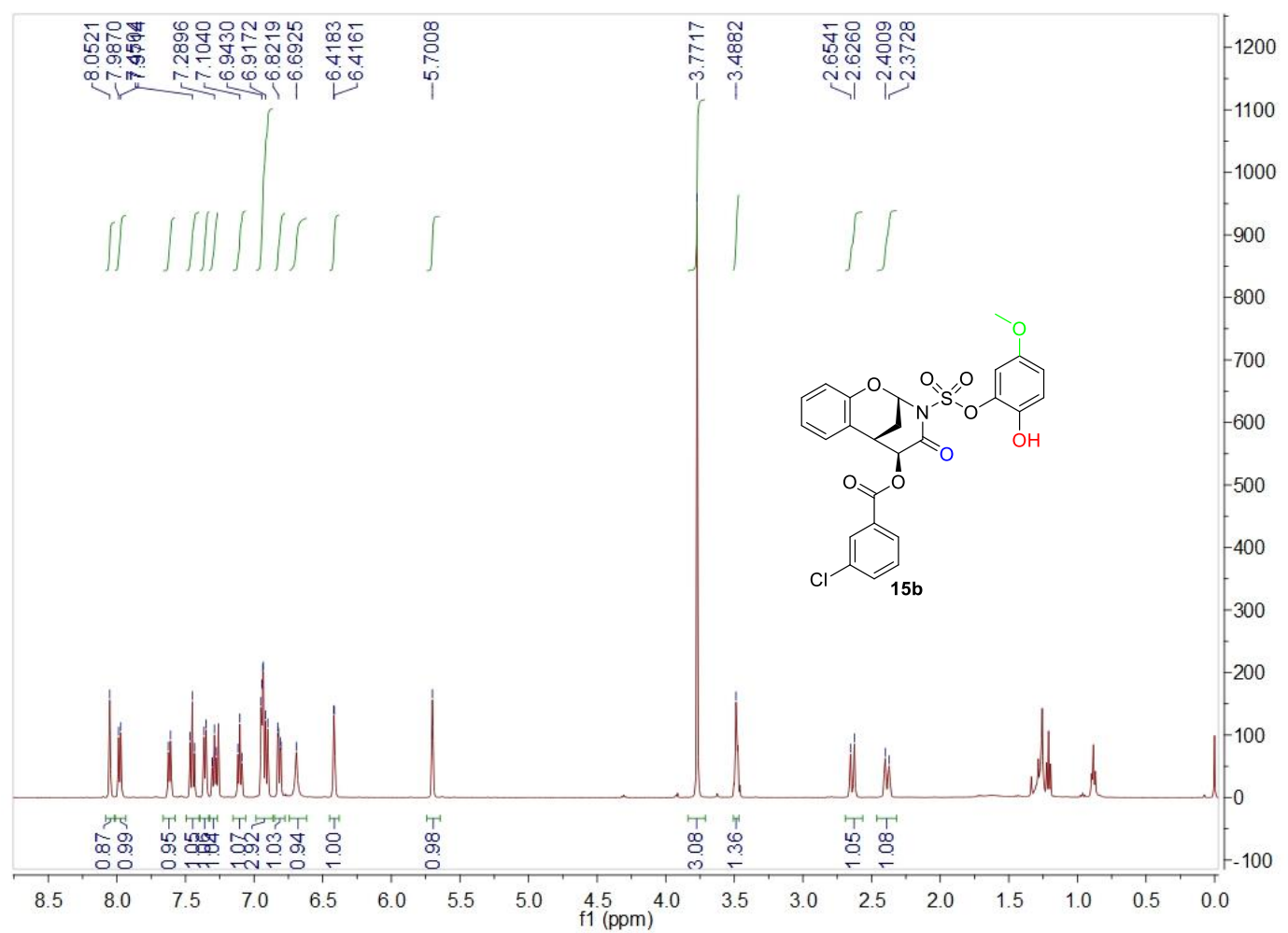

${ }^{13} \mathrm{C} \mathrm{NMR}$ of $15 b, \mathrm{CDCl}_{3}$

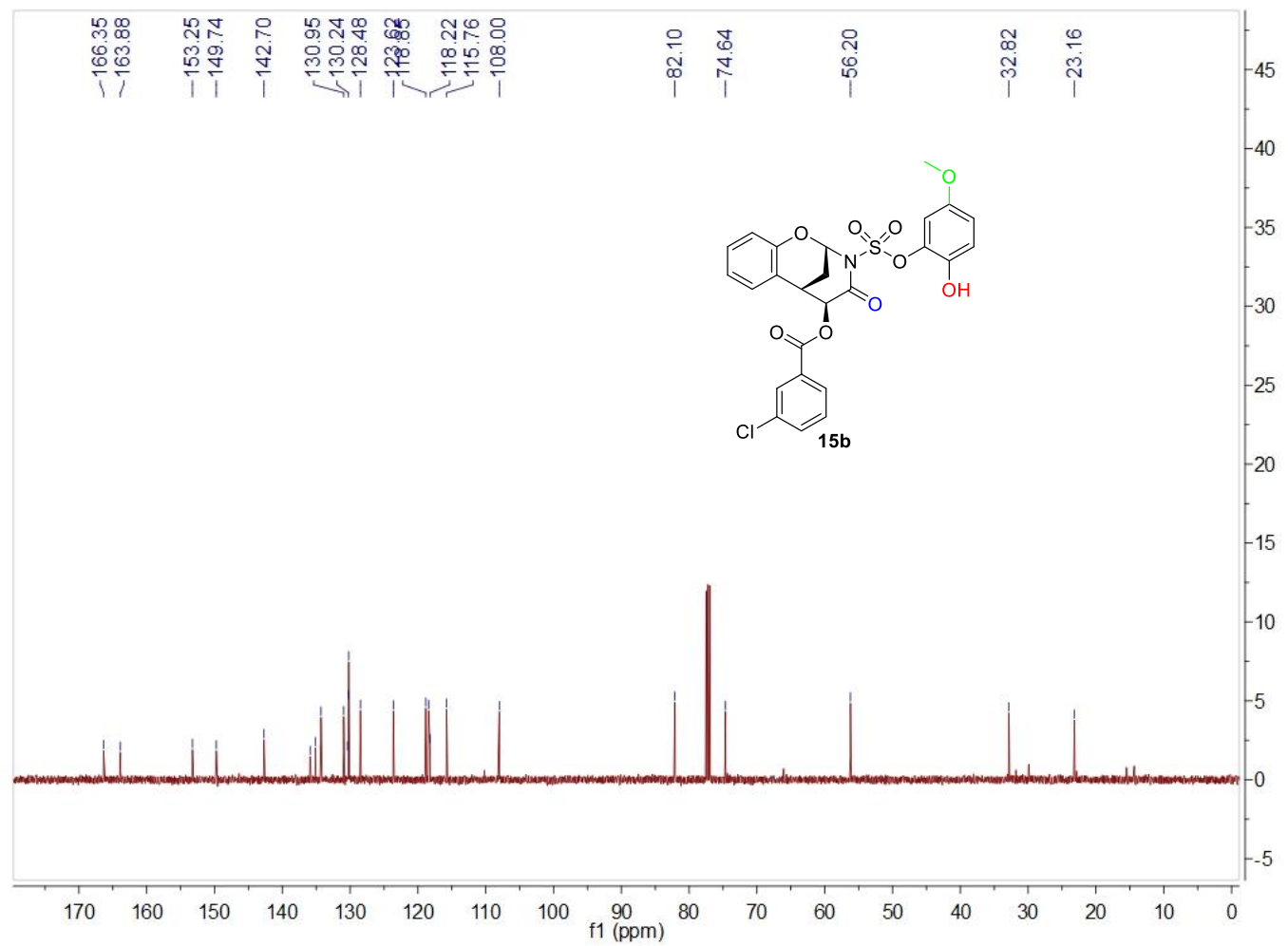




\section{Chiral HPLC analysis of rac-15b}

Chrom Type: Fixed WL Chromatogram, $225 \mathrm{~nm}$

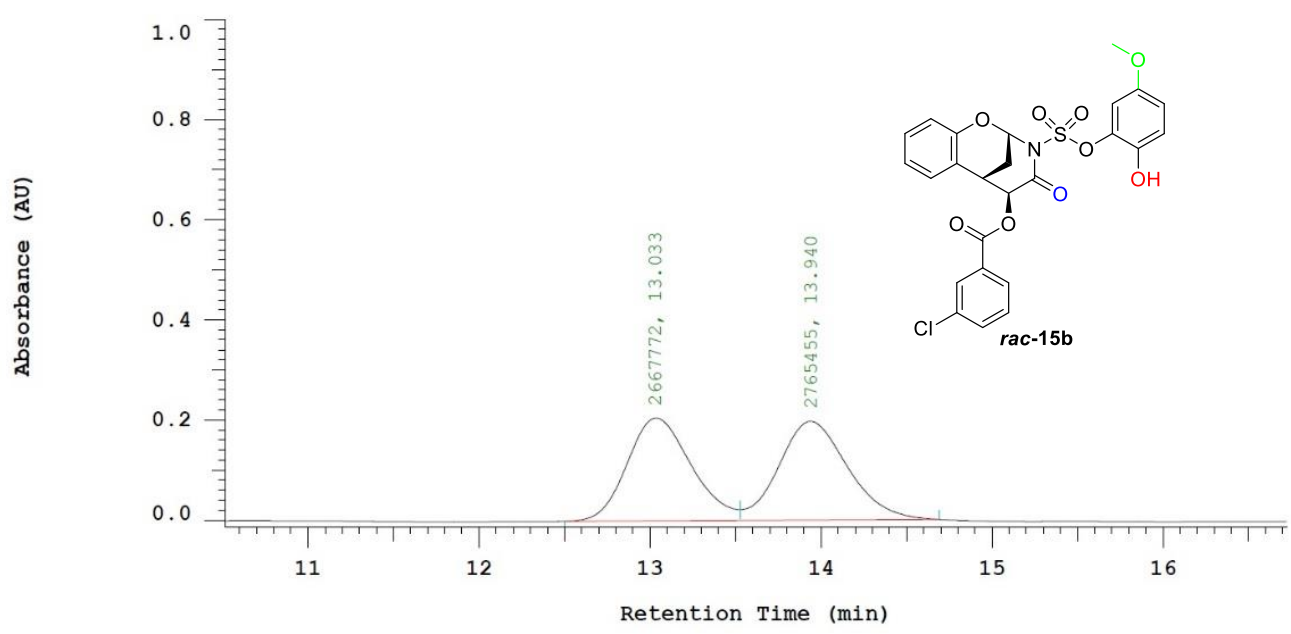

Chrom Type: Fixed WL Chromatogram, $225 \mathrm{~nm}$

Peak Quantitation: AREA

Calculation Method: AREA\%

\begin{tabular}{ccccc} 
No. & RT & Area & Area \% & BC \\
\hline 1 & 13.033 & 2667772 & 49.101 & BV \\
2 & 13.940 & 2765455 & 50.899 & VB \\
\hline & & 5433227 & 100.000 & \\
\hline
\end{tabular}

\section{Chiral HPLC analysis of 15b}

Chrom Type: Fixed WL Chromatogram, 225 nm

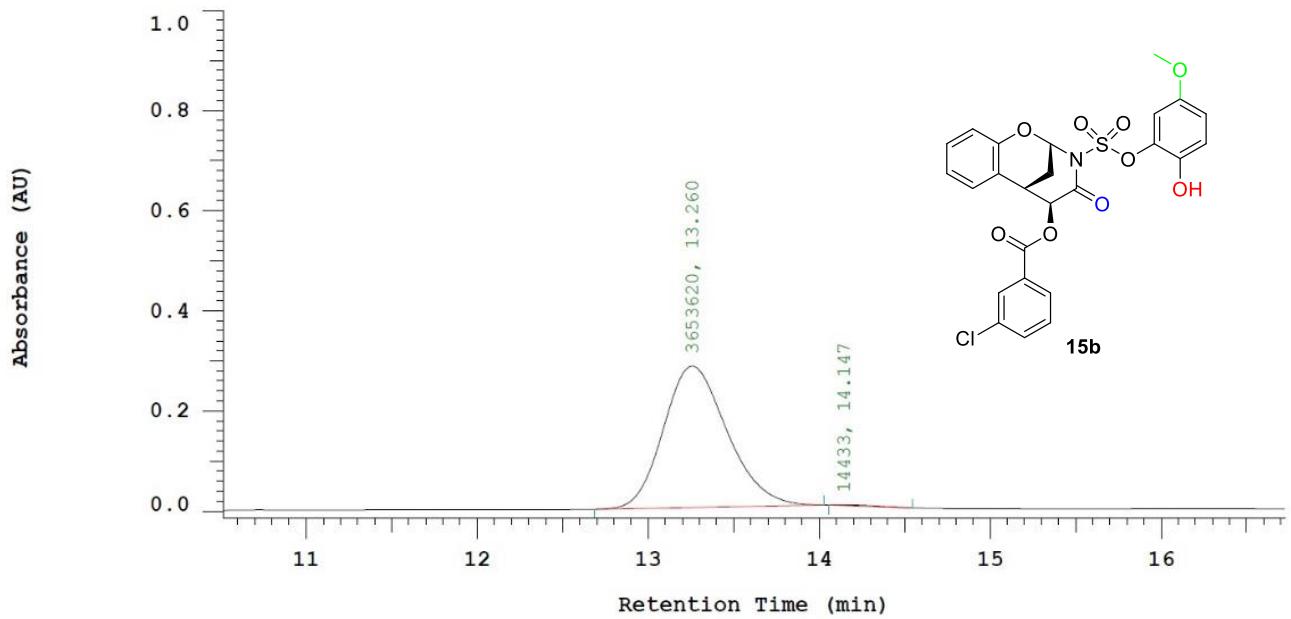

Chrom Type: Fixed WL Chromatogram, $225 \mathrm{~nm}$

Peak Quantitation: AREA

Calculation Method: AREA응

\begin{tabular}{|c|c|c|c|c|}
\hline No. & $\mathrm{RT}$ & Area & Area \% & $\mathrm{BC}$ \\
\hline 1 & 13.260 & 3653620 & 99.607 & BB \\
\hline 2 & 14.147 & 14433 & 0.393 & BB \\
\hline & & 3668053 & 100.000 & \\
\hline
\end{tabular}


${ }^{1} \mathrm{H}$ NMR of $15 \mathrm{~d}, \mathrm{CDCl}_{3}$

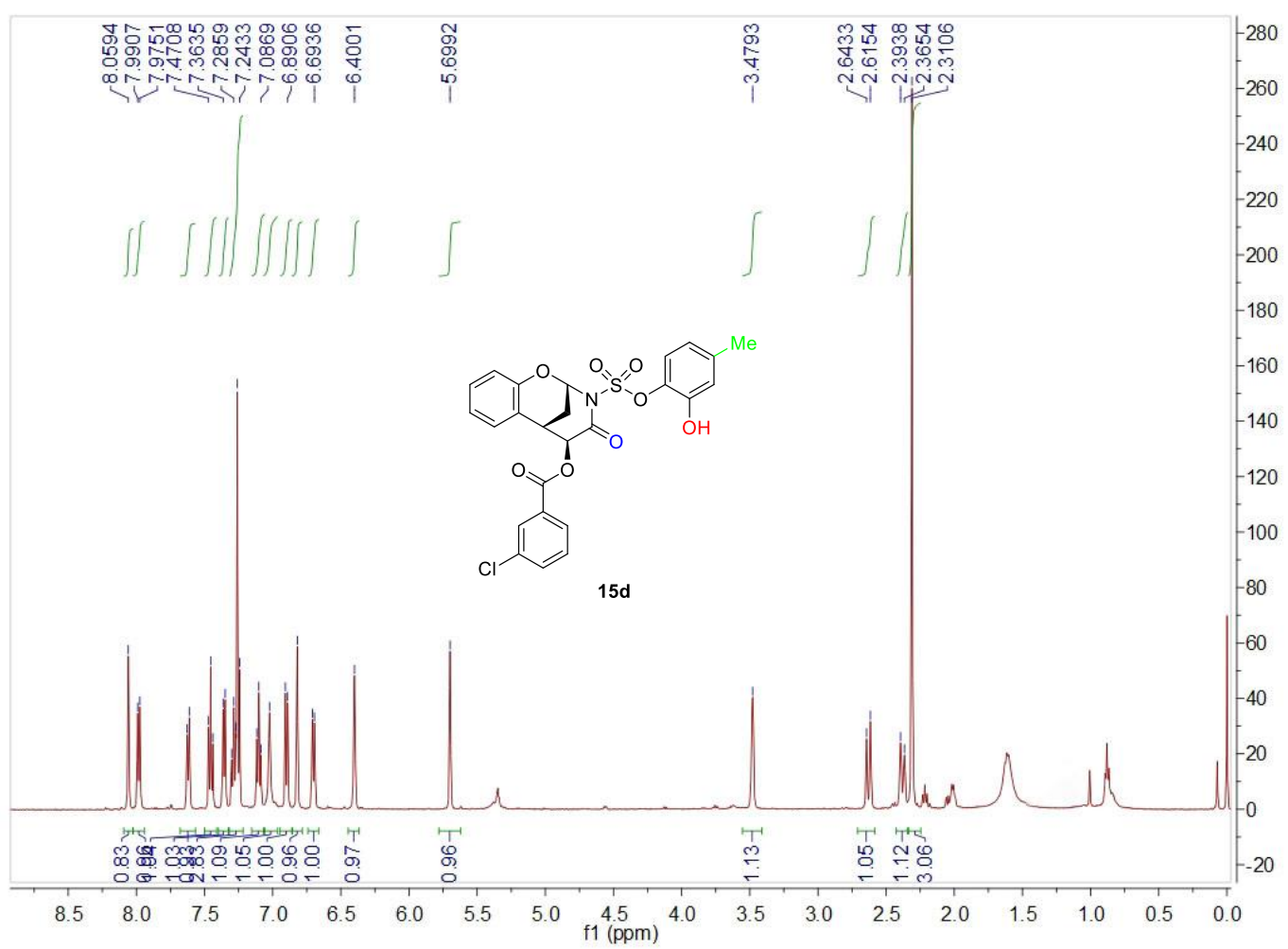

${ }^{13} \mathrm{C}$ NMR of $15 \mathrm{~d}, \mathrm{CDCl}_{3}$

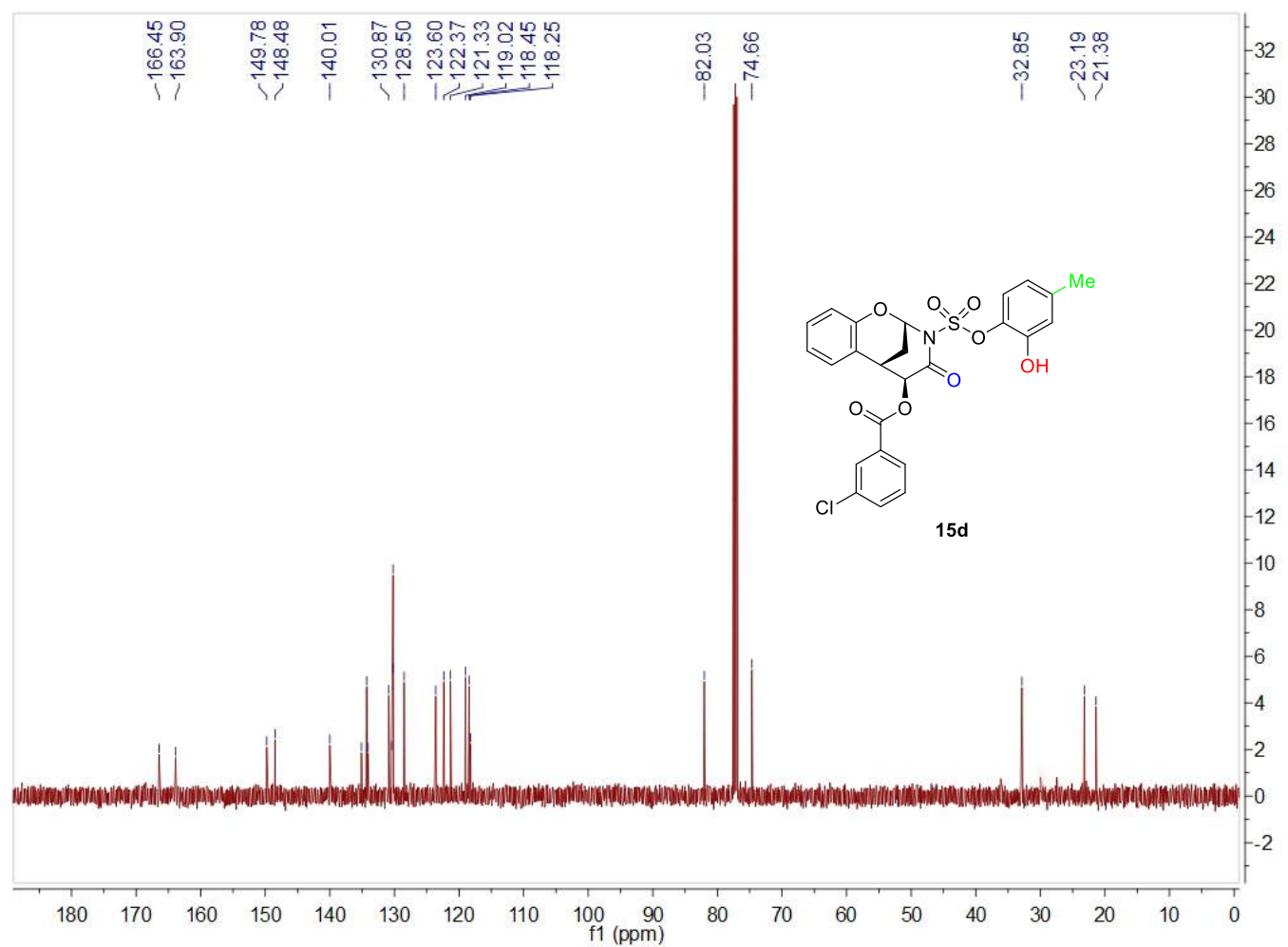




\section{Chiral HPLC analysis of rac-15d}

Chrom Type: Fixed WL Chromatogram, 210 nm

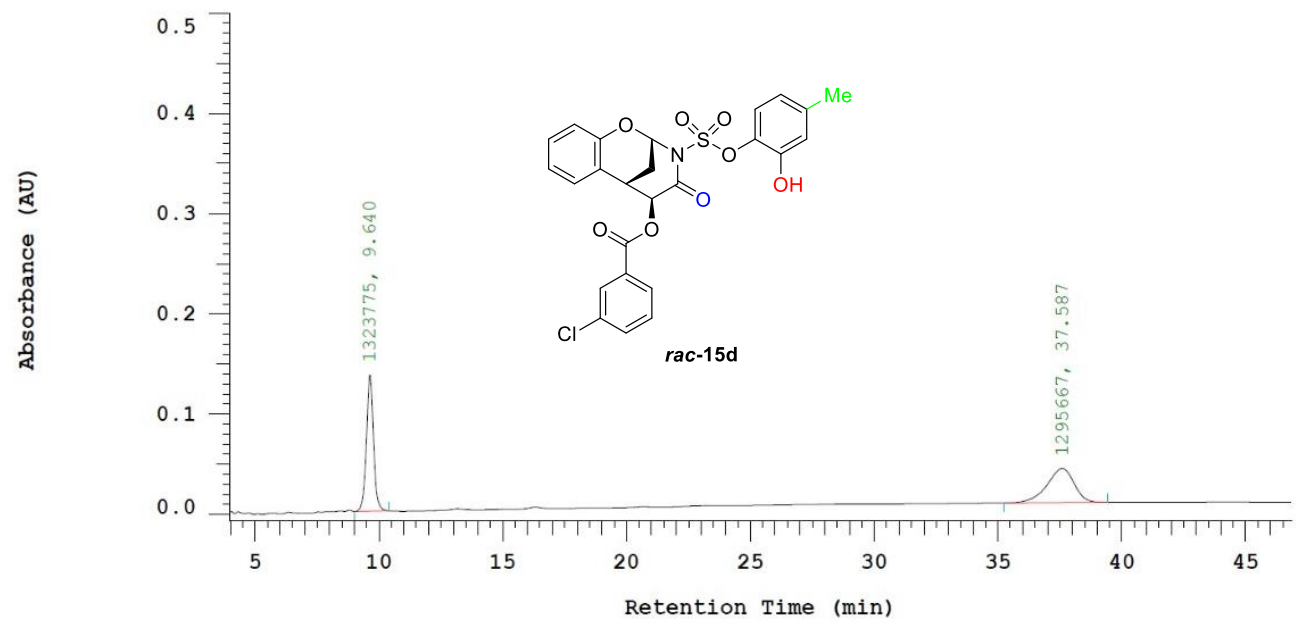

Chrom Type: Fixed WL Chromatogram, $210 \mathrm{~nm}$

Peak Quantitation: AREA

Calculation Method: AREA

\begin{tabular}{|c|c|c|c|}
\hline No. & $\mathrm{RT}$ & Area & Area \% \\
\hline 1 & 9.640 & 1323775 & 50.537 \\
\hline 2 & 37.587 & 1295667 & 49.463 \\
\hline & & 2619442 & 100.000 \\
\hline
\end{tabular}

\section{Chiral HPLC analysis of 15d}

Chrom Type: Fixed WL Chromatogram, $210 \mathrm{~nm}$

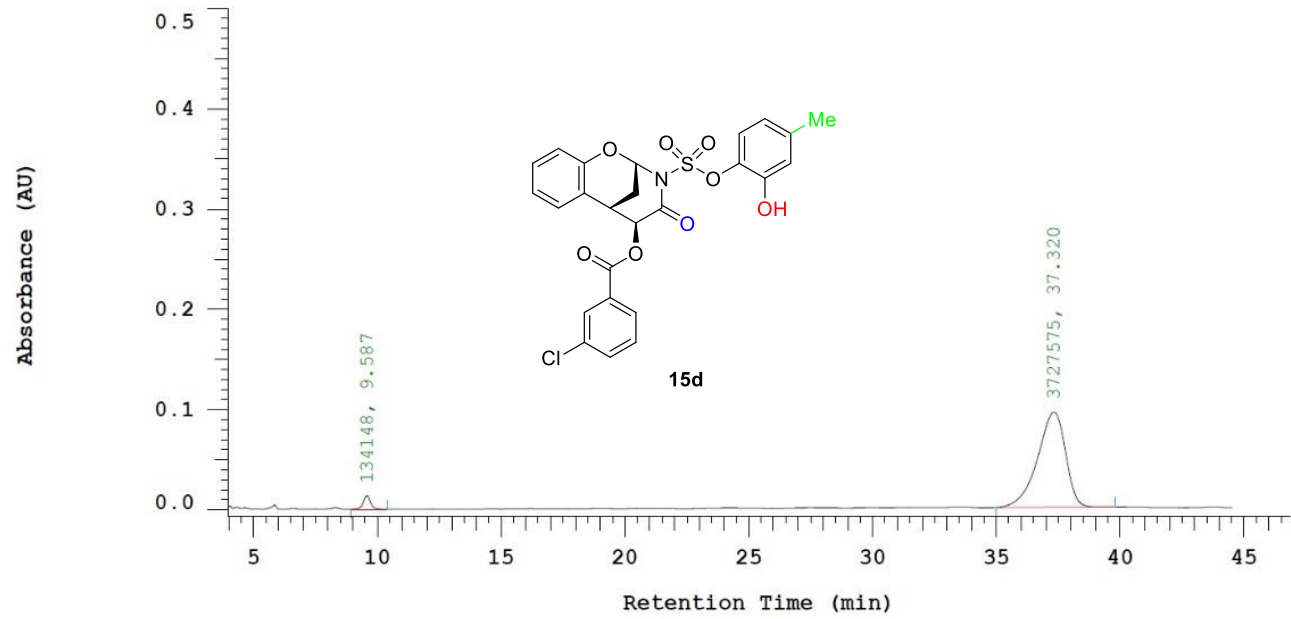

Chrom Type: Fixed WL Chromatogram, $210 \mathrm{~nm}$

Peak Quantitation: AREA

Calculation Method: AREA응

\begin{tabular}{|c|c|c|c|c|}
\hline No. & $\mathrm{RT}$ & Area & Area 응 & $\mathrm{BC}$ \\
\hline 1 & 9.587 & 134148 & 3.474 & BB \\
\hline 2 & 37.320 & 3727575 & 96.526 & $\mathrm{BB}$ \\
\hline & & 3861723 & 100.000 & \\
\hline
\end{tabular}


${ }^{1} \mathrm{H}$ NMR of $15 e, \mathrm{CDCl}_{3}$

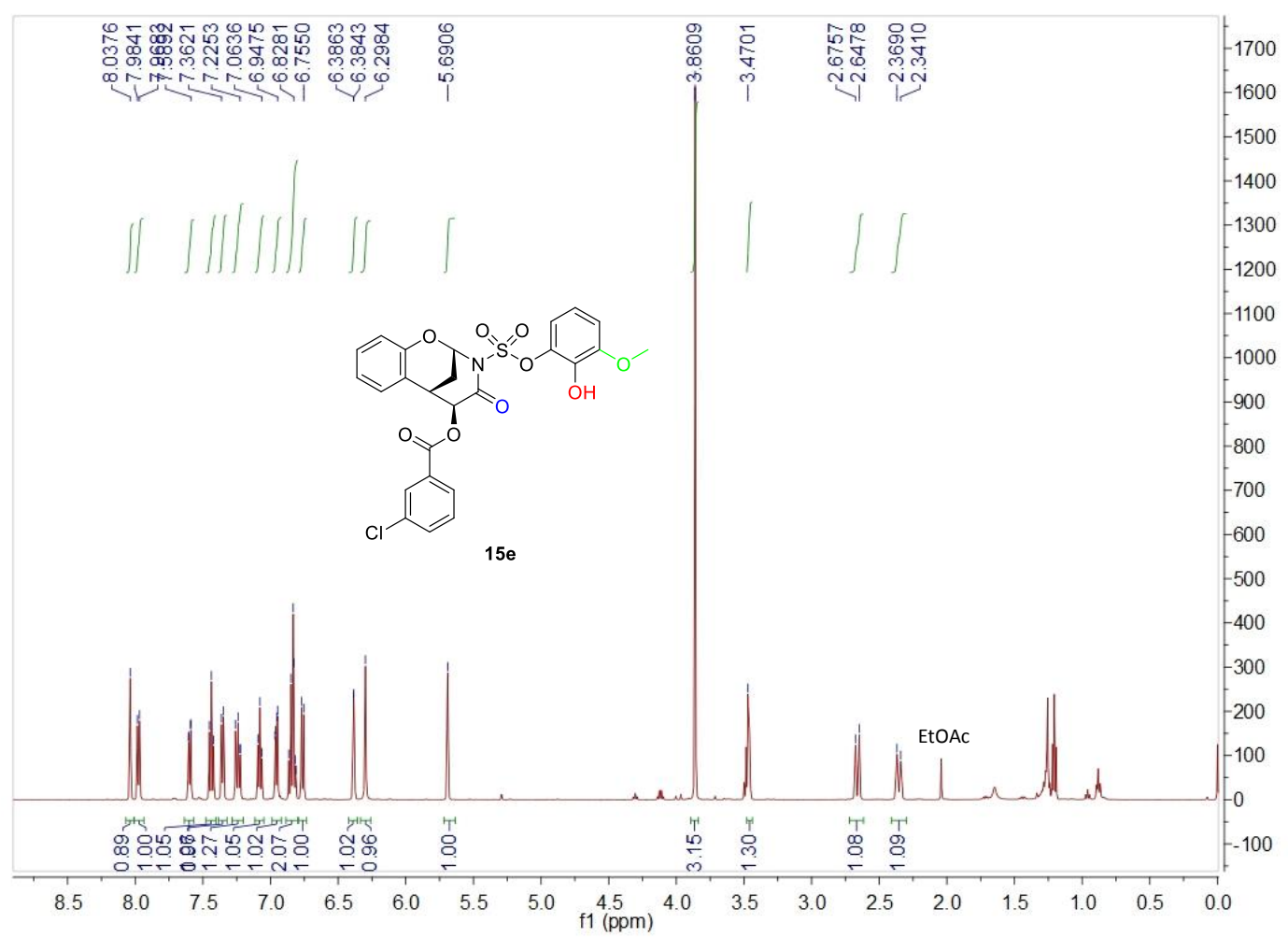

${ }^{13} \mathrm{CNMR}$ of $15 \mathrm{e}, \mathrm{CDCl}_{3}$

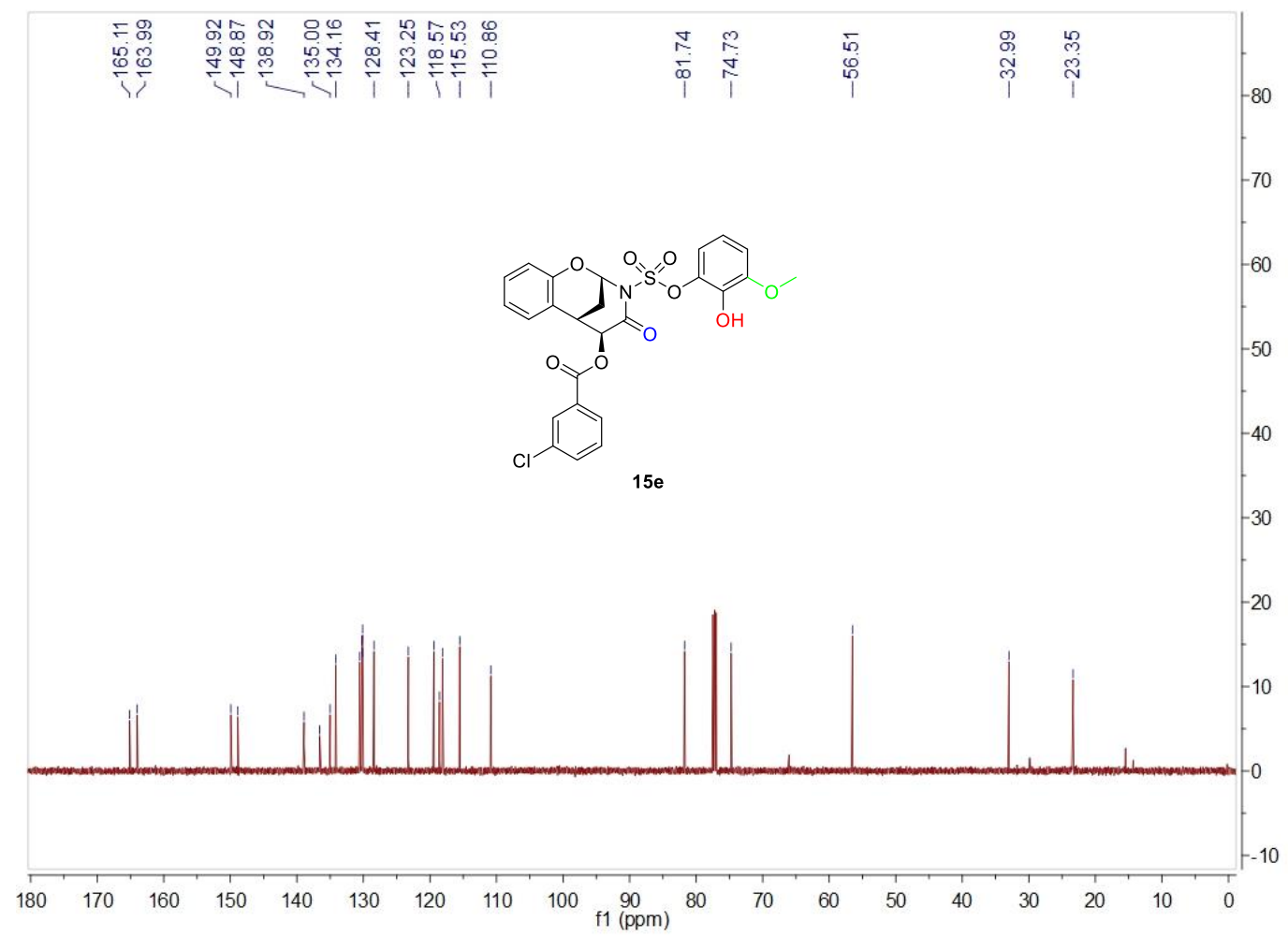




\section{Chiral HPLC analysis of rac-15e}

Chrom Type: Fixed WL Chromatogram, 210 nm

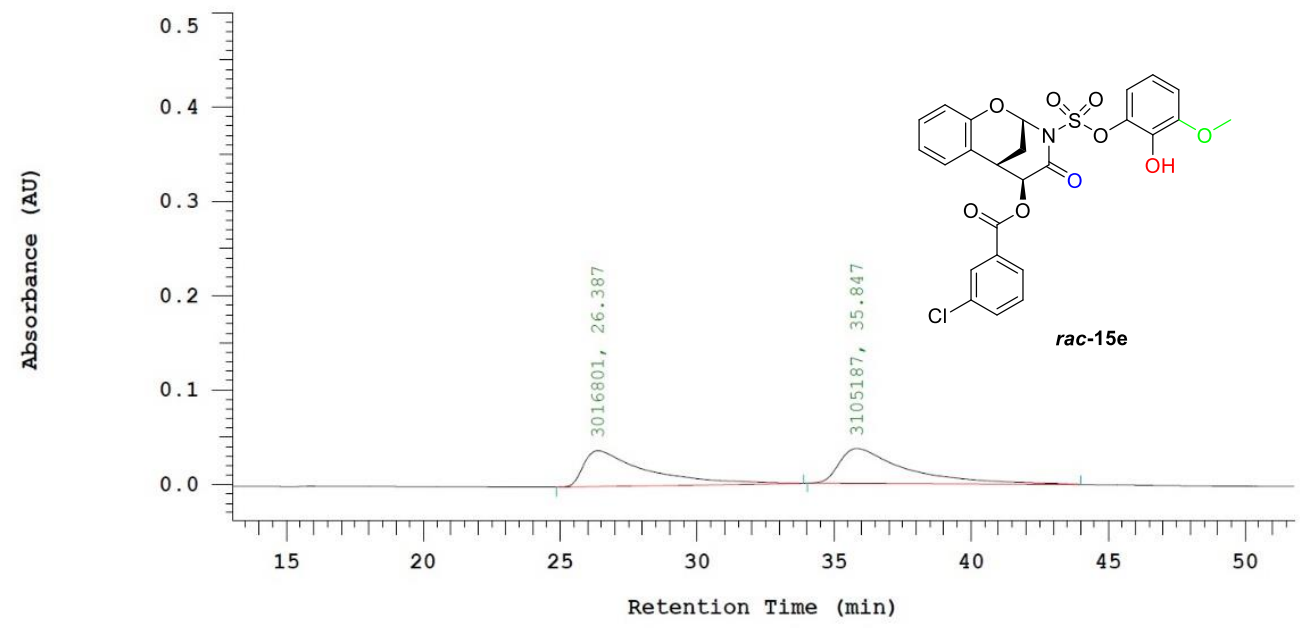

Chrom Type: Fixed WL Chromatogram, $210 \mathrm{~nm}$

Peak Quantitation: AREA

Calculation Method: AREA\%

\begin{tabular}{ccccc} 
No. & RT & Area & Area & BC \\
\hline 1 & 26.387 & 3016801 & 49.278 & BB \\
2 & 35.847 & 3105187 & 50.722 & BB \\
\hline & 6121988 & 100.000 & \\
\hline
\end{tabular}

\section{Chiral HPLC analysis of 15e}

Chrom Type: Fixed WL Chromatogram, $210 \mathrm{~nm}$

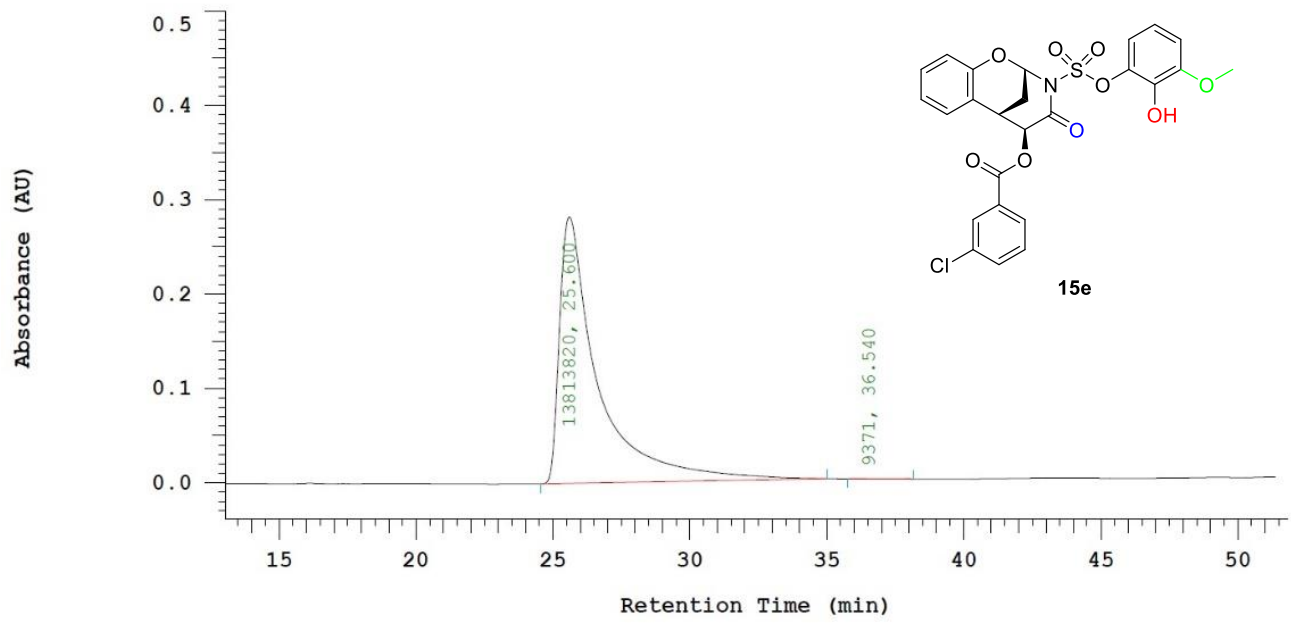

Chrom Type: Fixed WL Chromatogram, $210 \mathrm{~nm}$

Peak Quantitation: AREA

Calculation Method: AREA응

\begin{tabular}{|c|c|c|c|c|}
\hline No. & $\mathrm{RT}$ & Area & Area 응 & $\mathrm{BC}$ \\
\hline 1 & 25.600 & 13813820 & 99.932 & $\mathrm{BB}$ \\
\hline 2 & 36.540 & 9371 & 0.068 & $\mathrm{BB}$ \\
\hline & & 13823191 & 100.000 & \\
\hline
\end{tabular}


${ }^{1} \mathrm{H}$ NMR of $20 \mathrm{~d}, \mathrm{CDCl}_{3}$

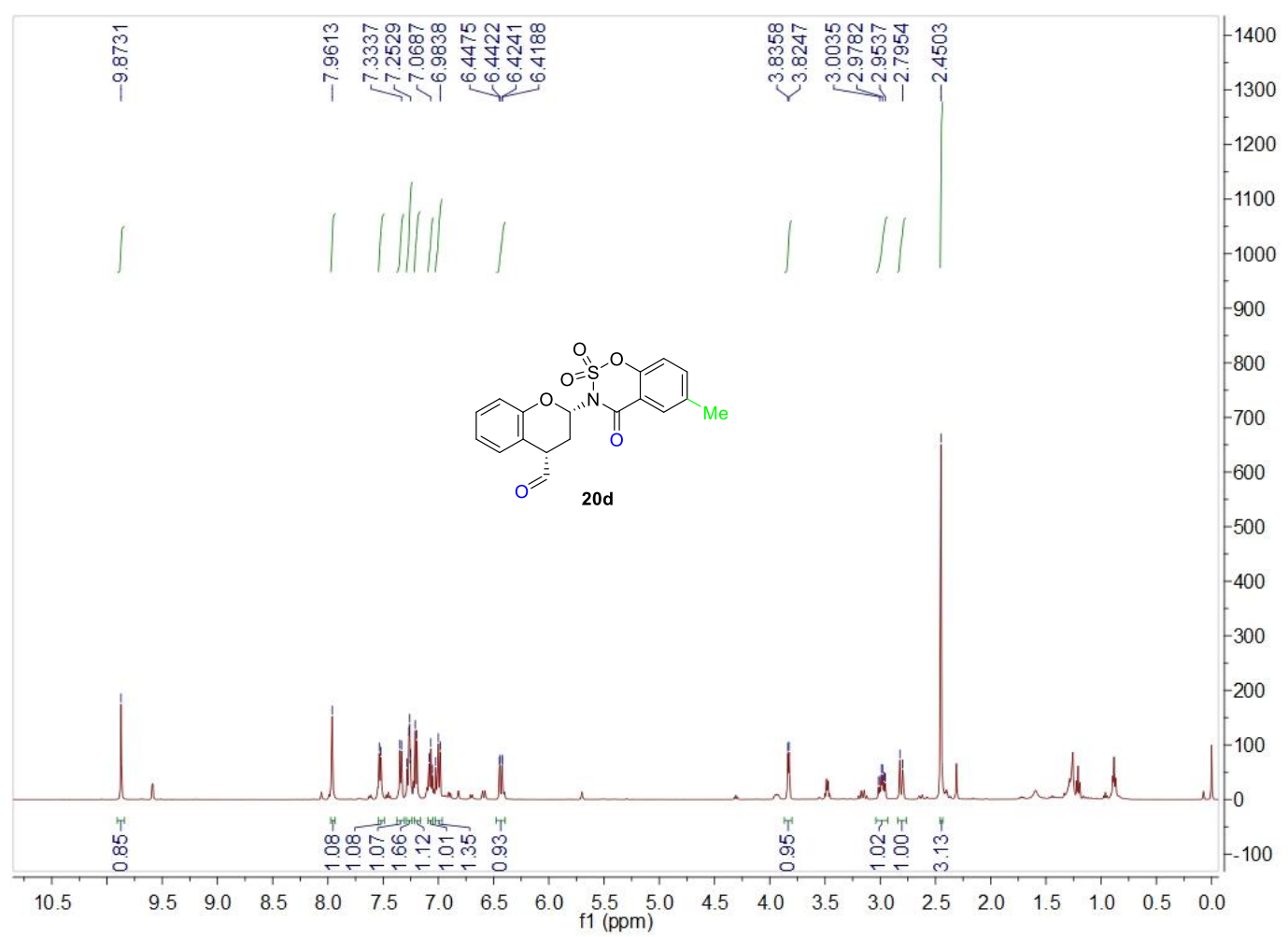

${ }^{13} \mathrm{C}$ NMR of $20 \mathrm{~d}, \mathrm{CDCl}_{3}$

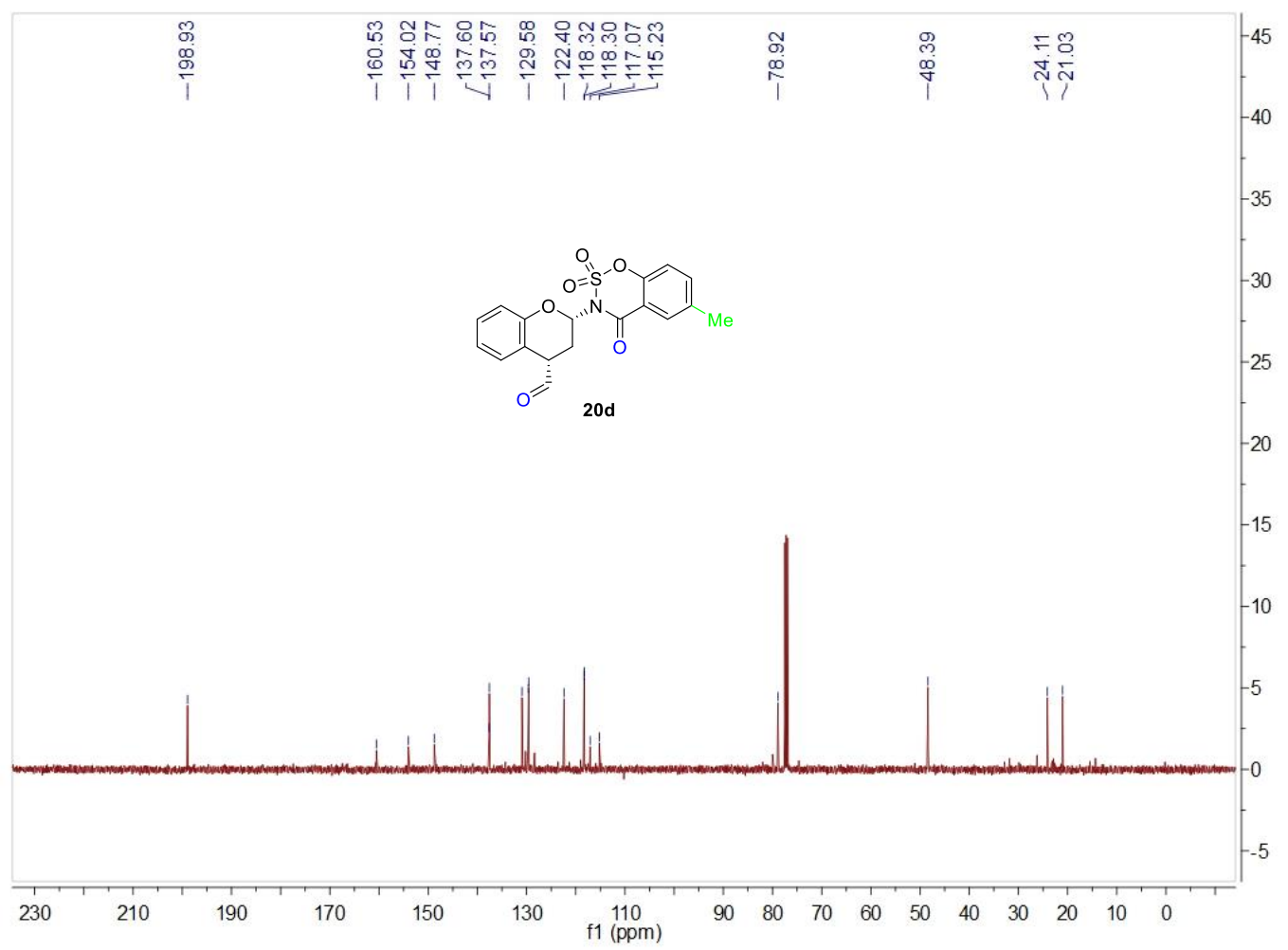


${ }^{1} \mathrm{H}$ NMR of $9 \mathrm{a}, \mathrm{CDCl}_{3}$

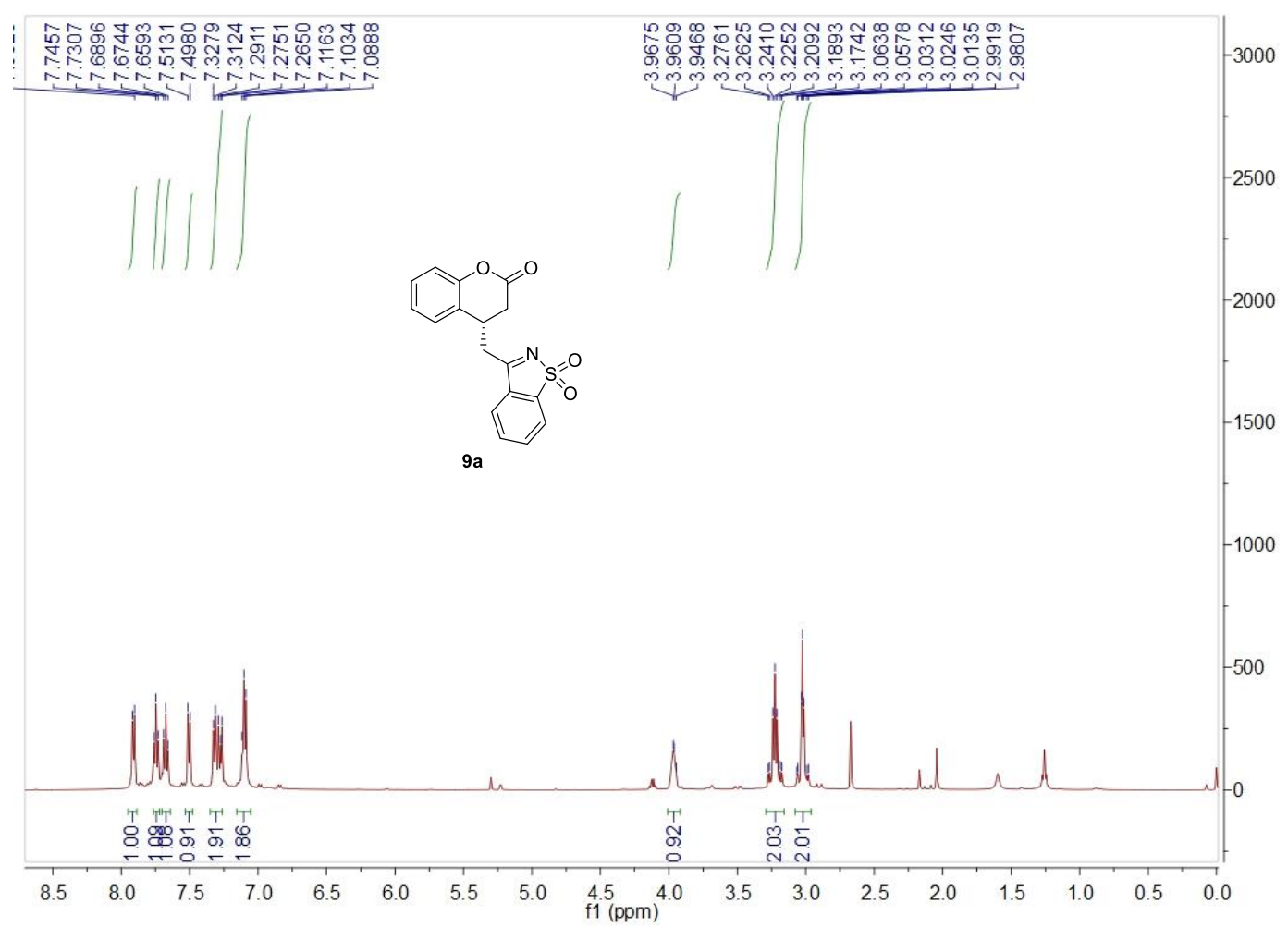

${ }^{13} \mathrm{C}$ NMR of $9 a, \mathrm{CDCl}_{3}$

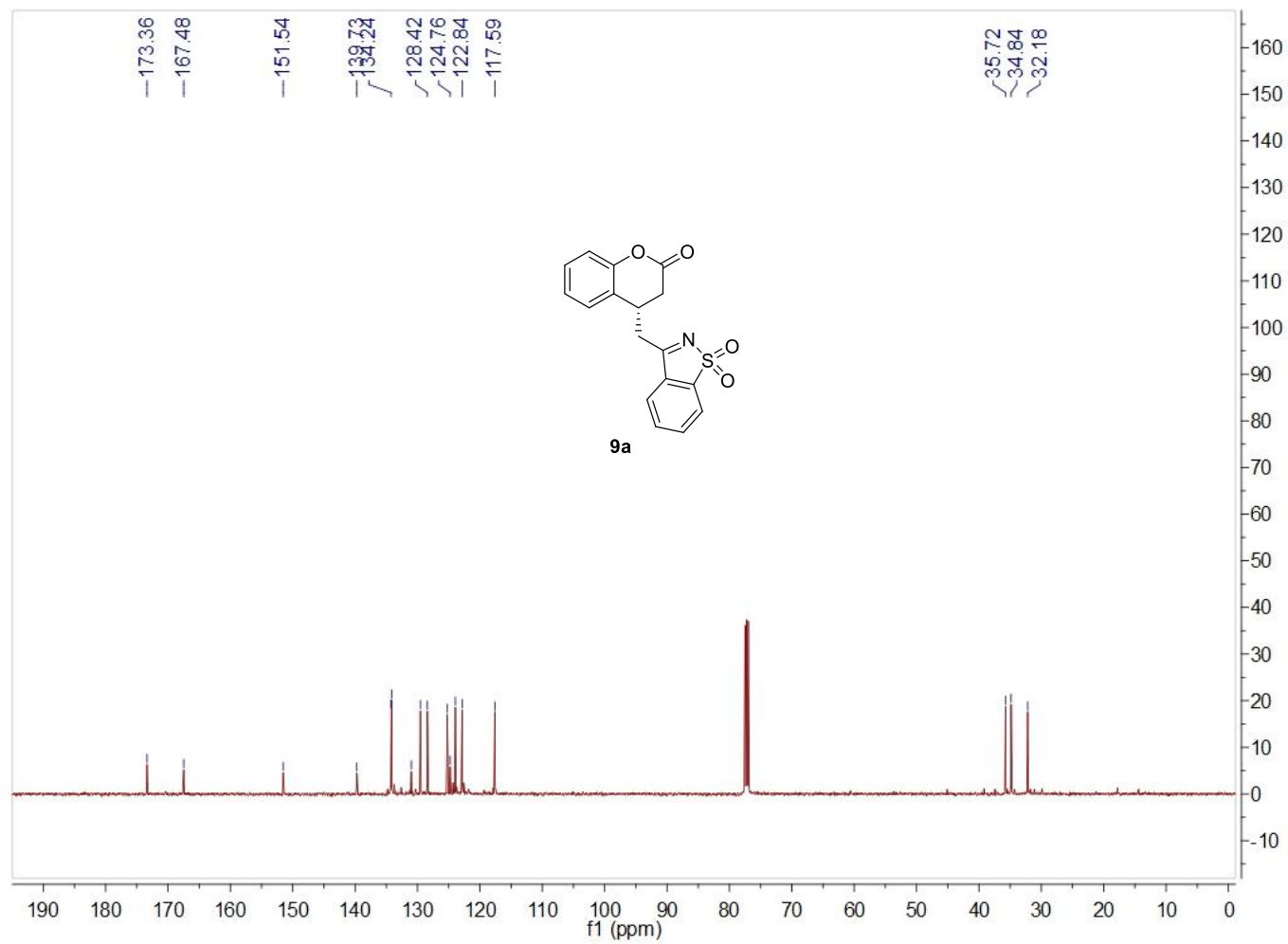




\section{Chiral HPLC analysis of rac-9a}

Chrom Type: Fixed WL Chromatogram, 225 nm

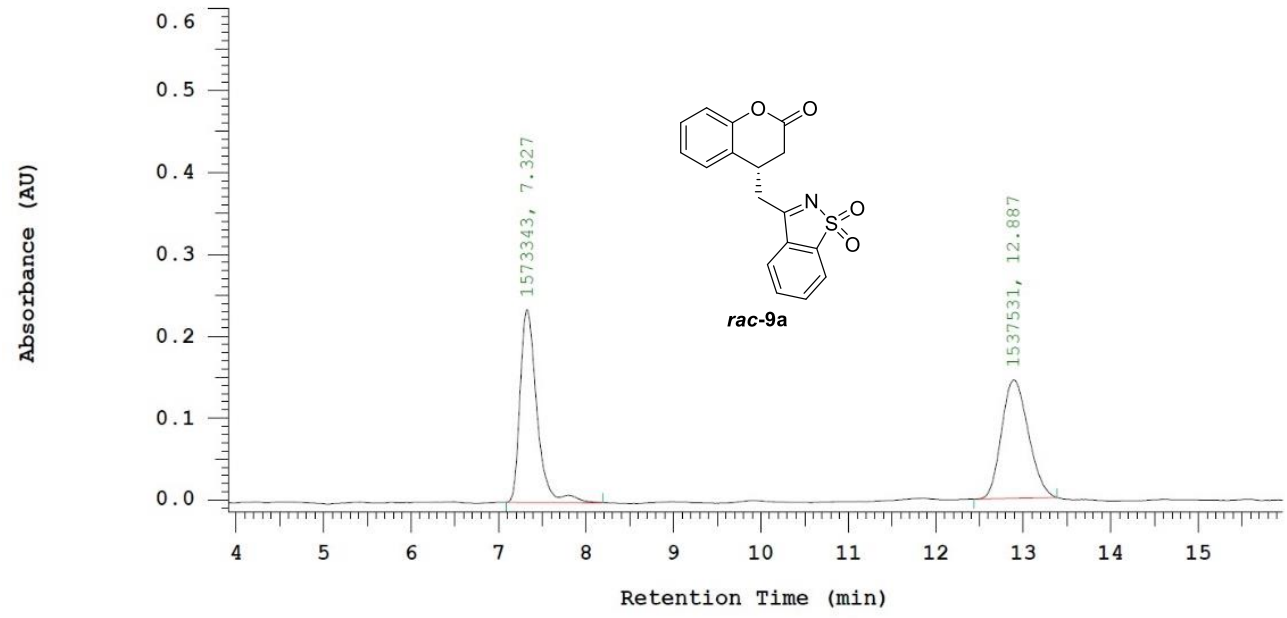

Chrom Type: Fixed WL Chromatogram, 225 nm Peak Quantitation: AREA

Calculation Method: AREA응

\begin{tabular}{rrrrr} 
No. & \multicolumn{1}{c}{ RT } & Area & Area $\%$ & BC \\
\hline 1 & 7.327 & 1573343 & 50.576 & BB \\
2 & 12.887 & 1537531 & 49.424 & BB \\
\hline & & 3110874 & 100.000 &
\end{tabular}

\section{Chiral HPLC analysis of 9a}

Chrom Type: Fixed WL Chromatogram, 225 nm

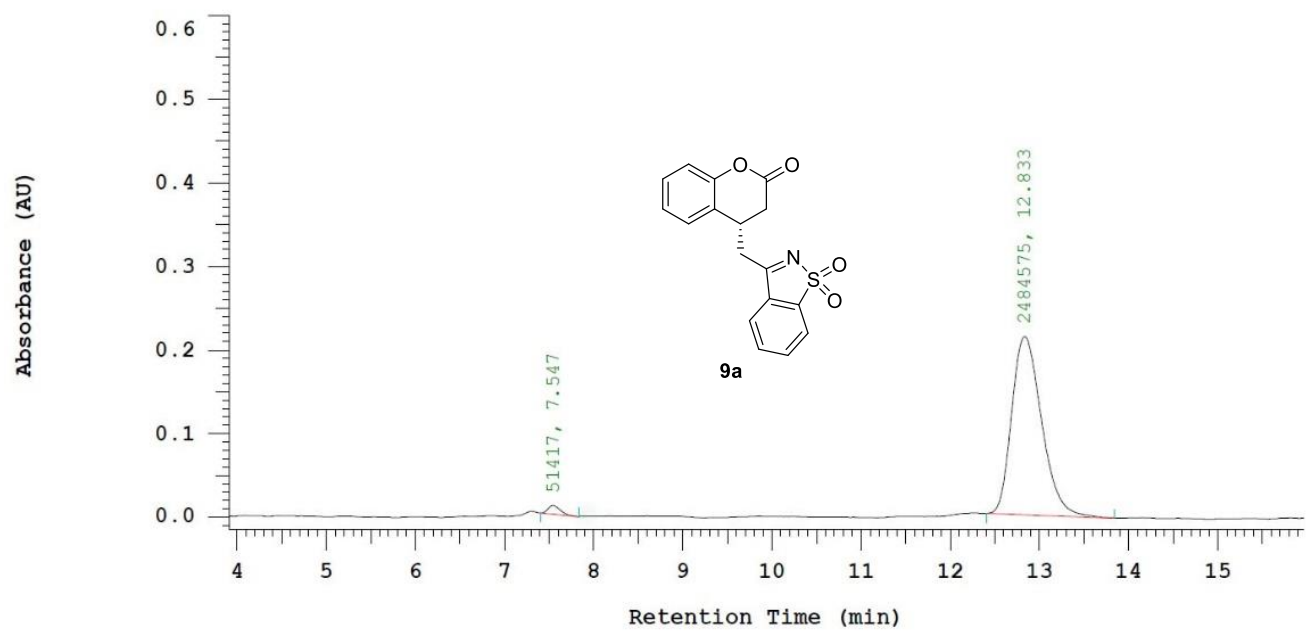

Chrom Type: Fixed WL Chromatogram, $225 \mathrm{~nm}$

Peak Quantitation: AREA

Calculation Method: AREA응

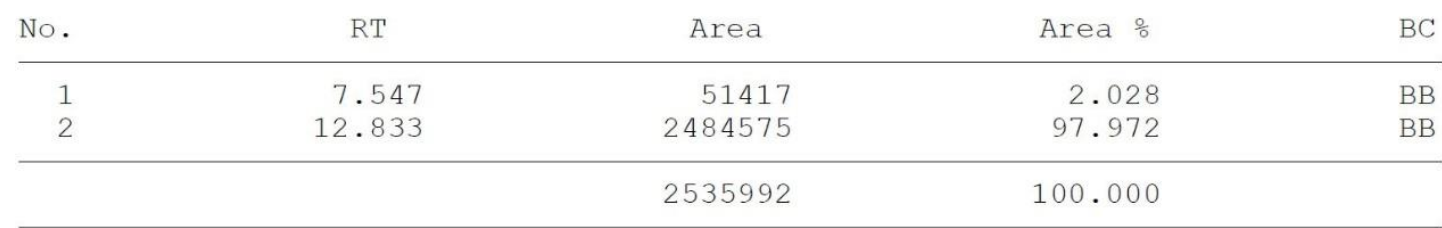


${ }^{1} \mathrm{H}$ NMR of $16, \mathrm{CDCl}_{3}$

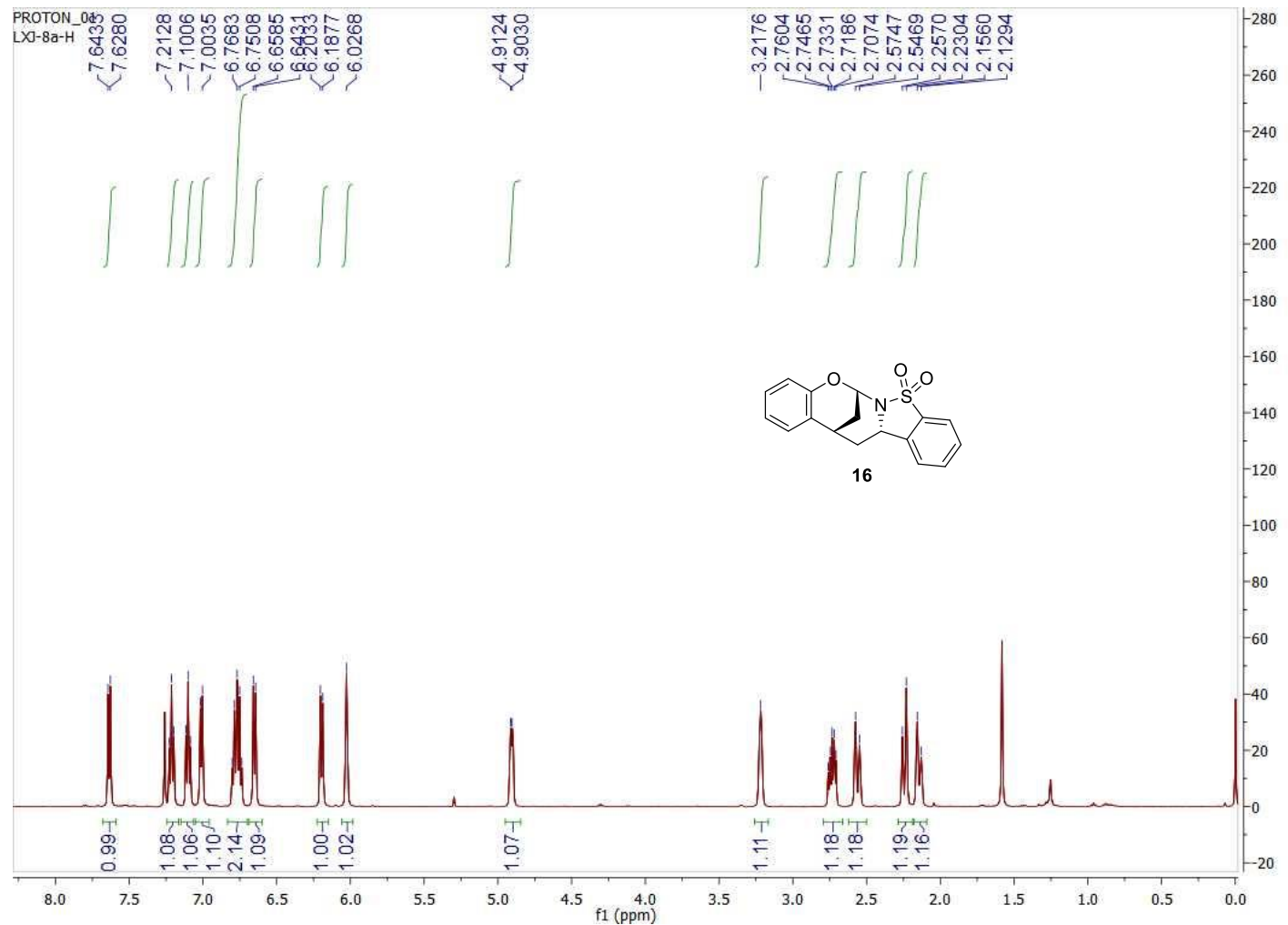

${ }^{13} \mathrm{C} \mathrm{NMR}$ of $16, \mathrm{CDCl}_{3}$

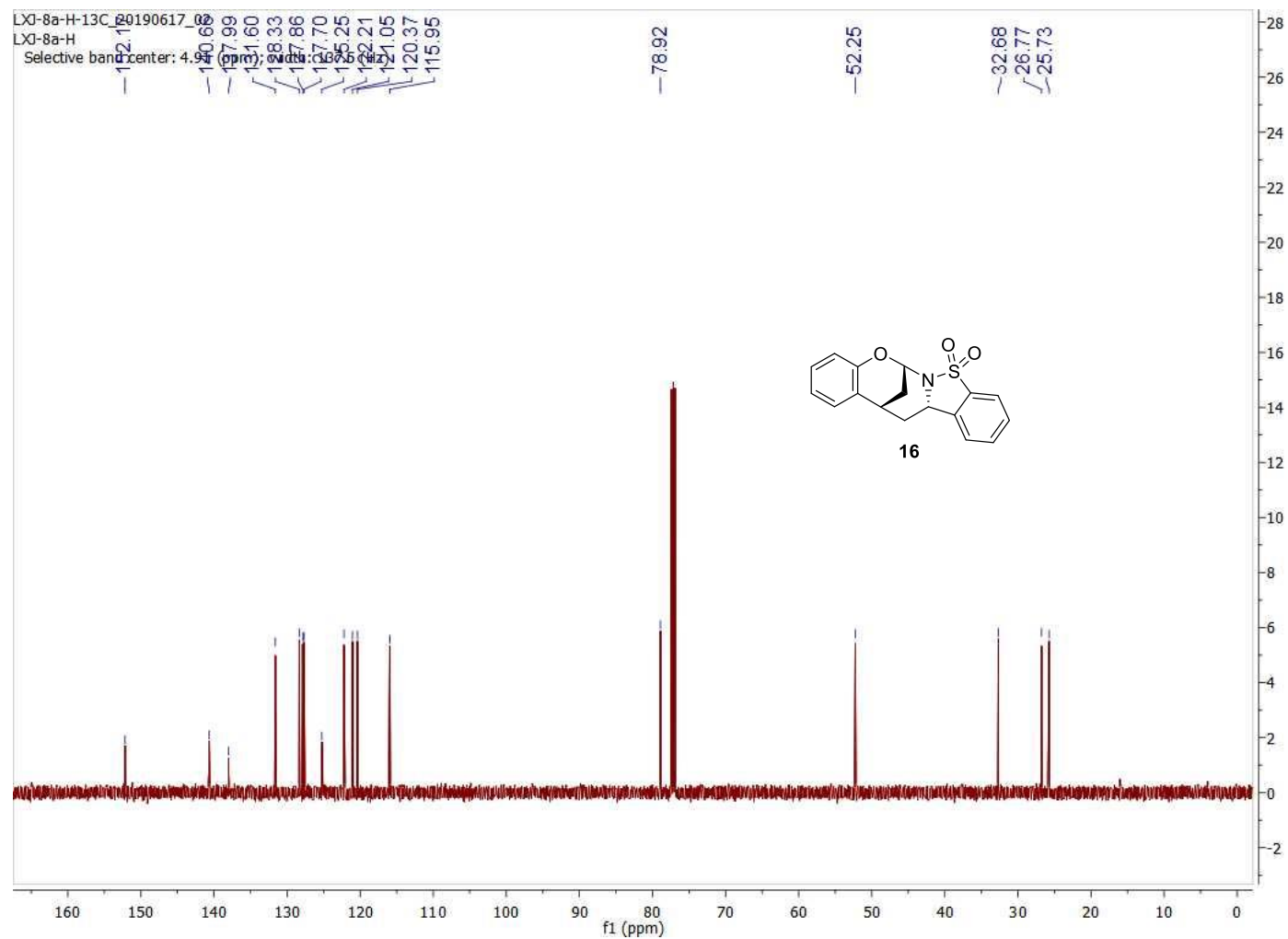


${ }^{1} \mathrm{H}-{ }^{1} \mathrm{H} \cos Y$ of $16, \mathrm{CDCl}_{3}$

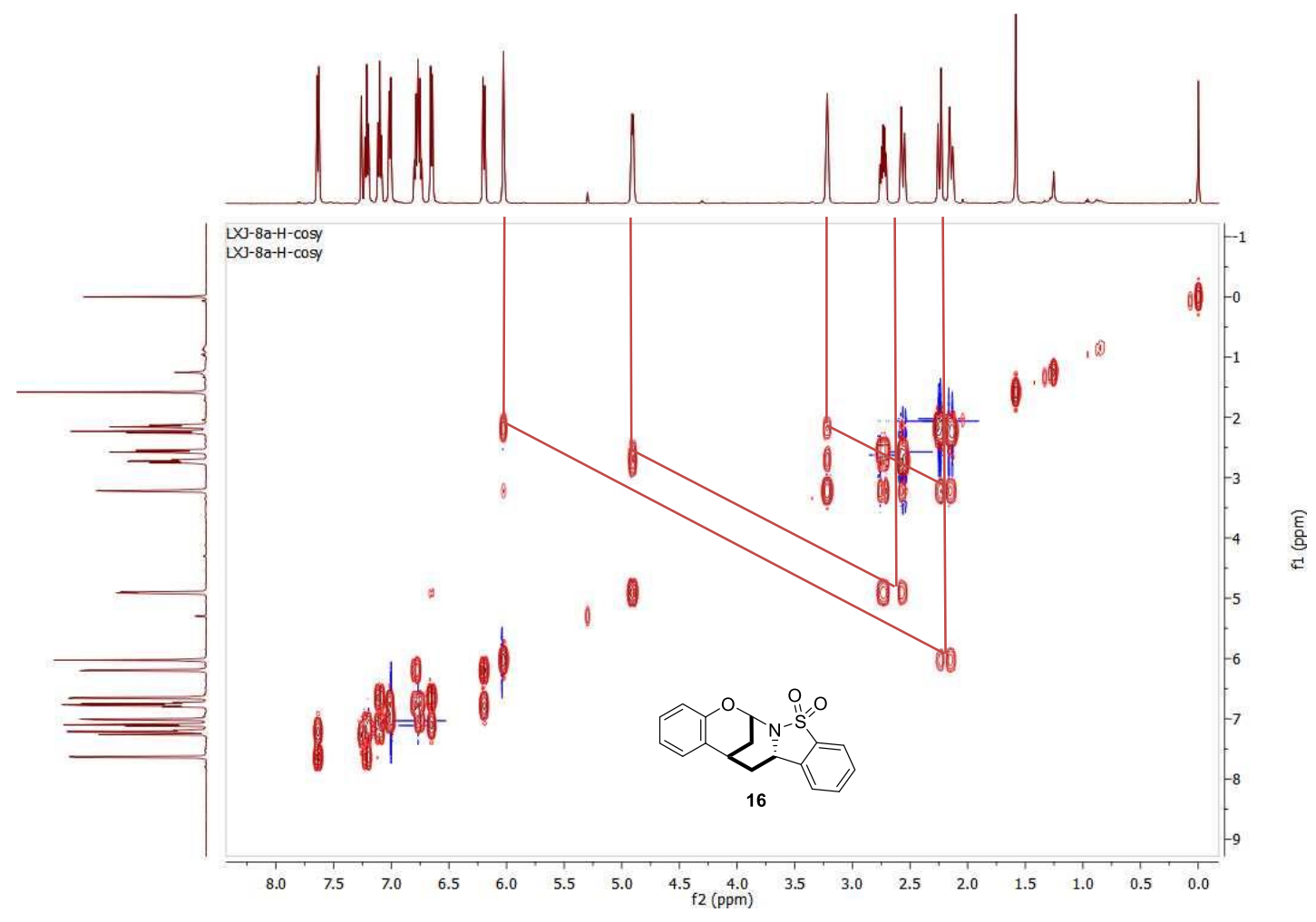

NOE spectrum of $16, \mathrm{CDCl}_{3}$

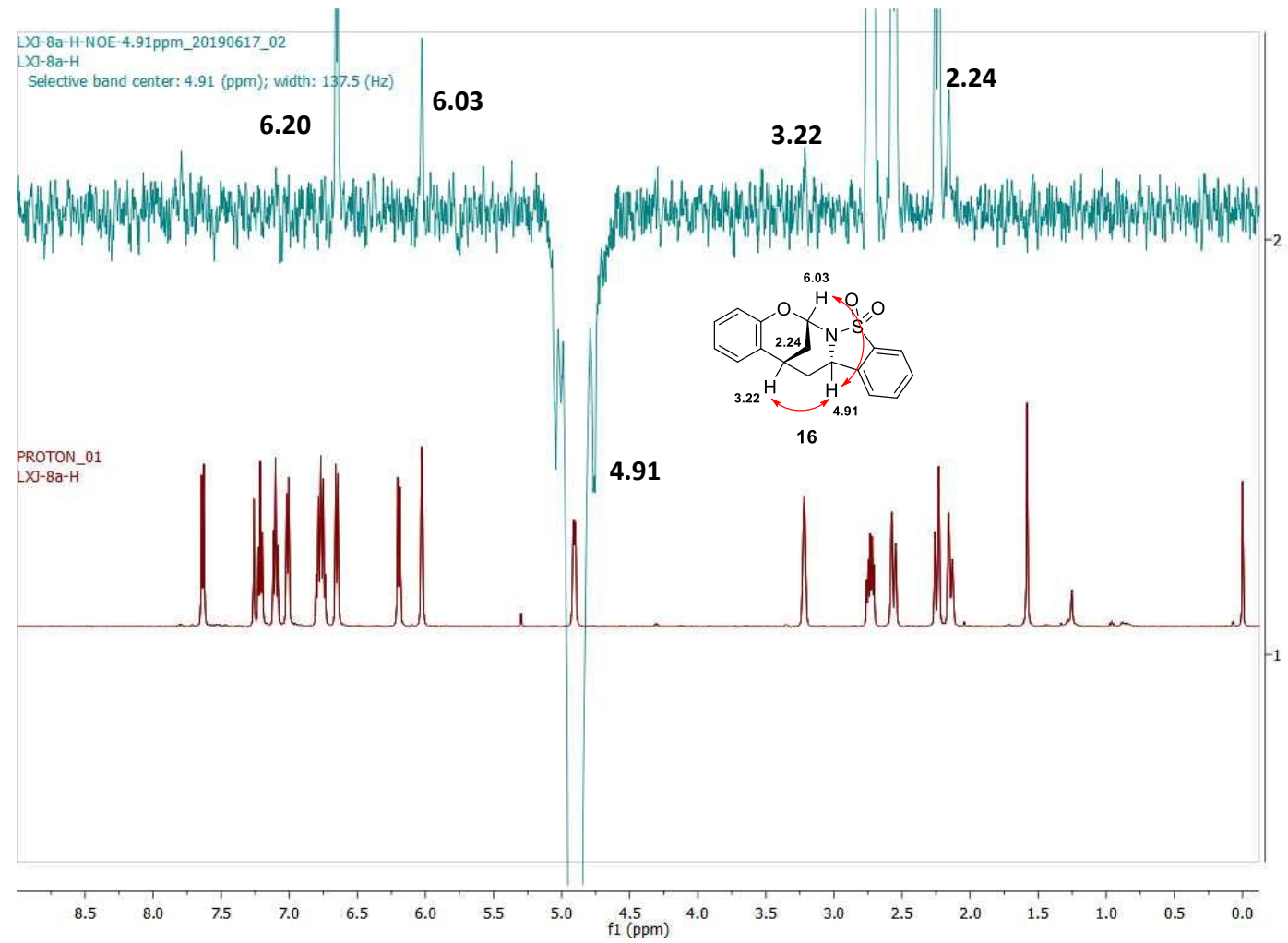


${ }^{1} \mathrm{H}$ NMR of $17, \mathrm{CDCl}_{3}$

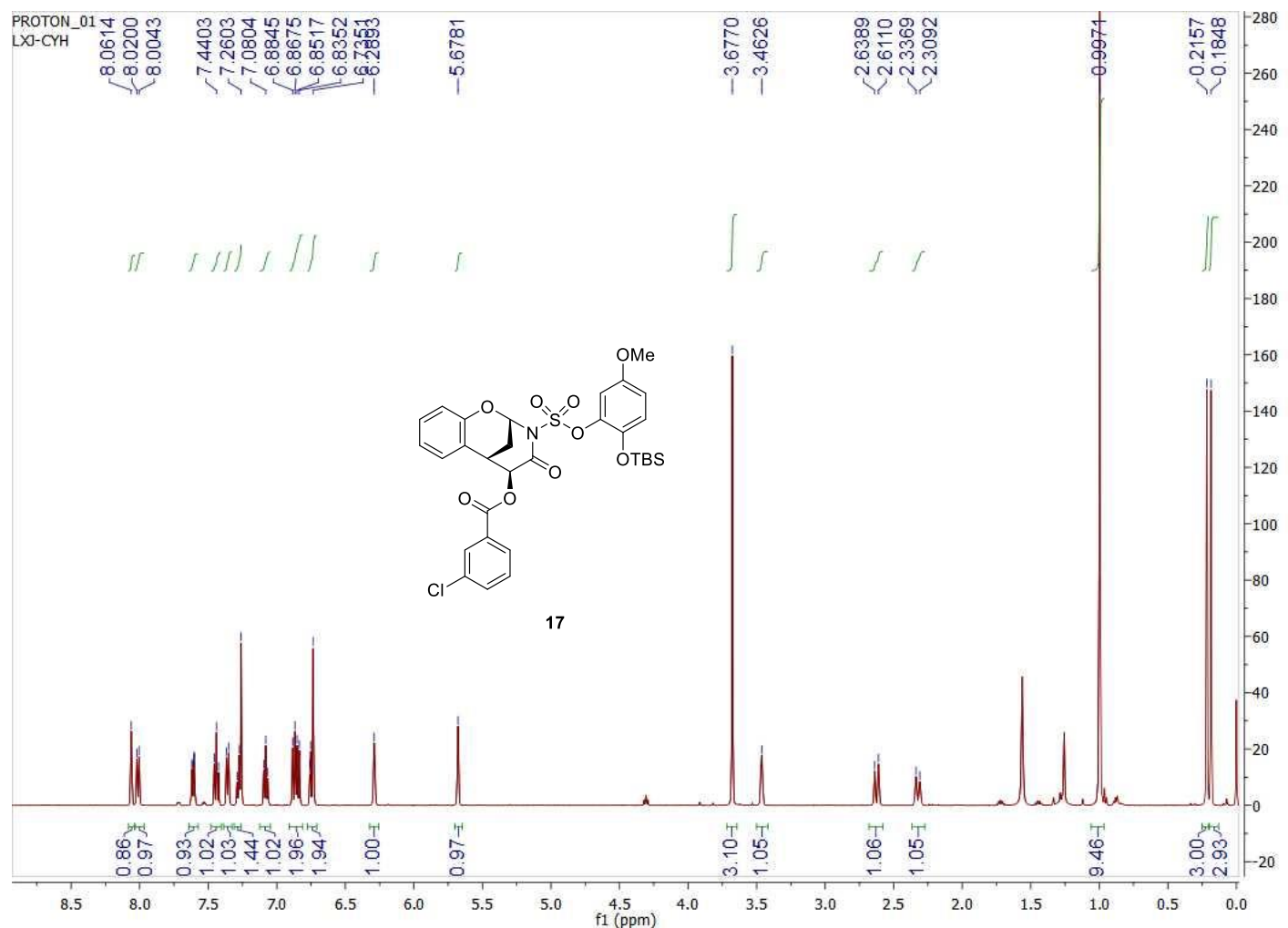

${ }^{13} \mathrm{C}$ NMR of $17, \mathrm{CDCl}_{3}$

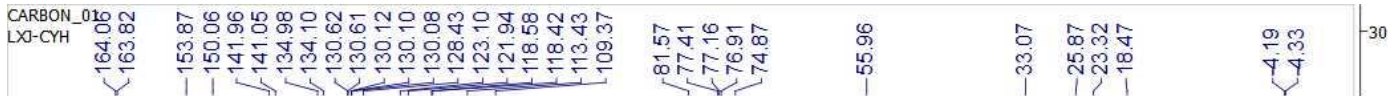

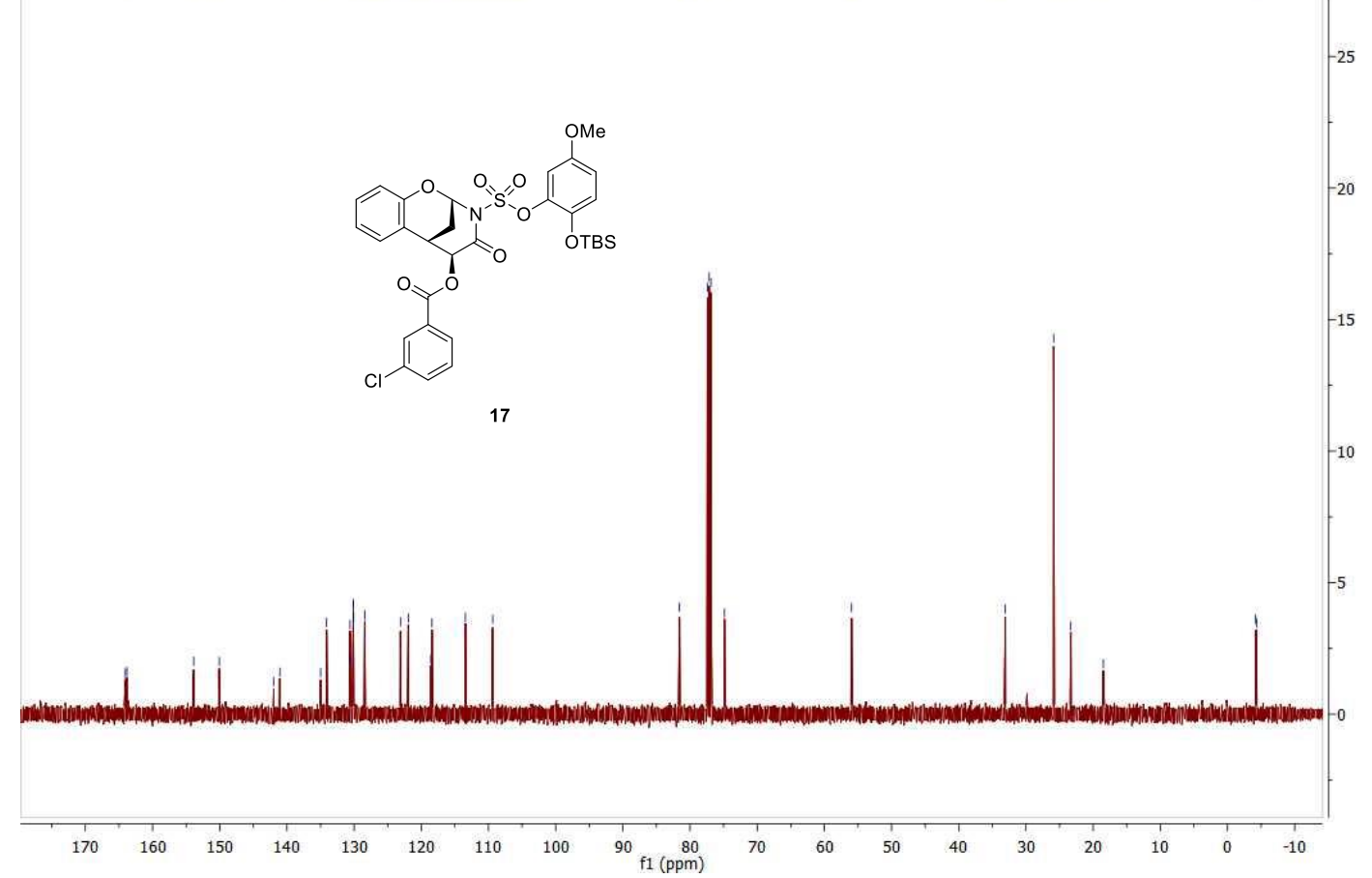


${ }^{1} \mathrm{H}$ NMR of $21, \mathrm{CDCl}_{3}$

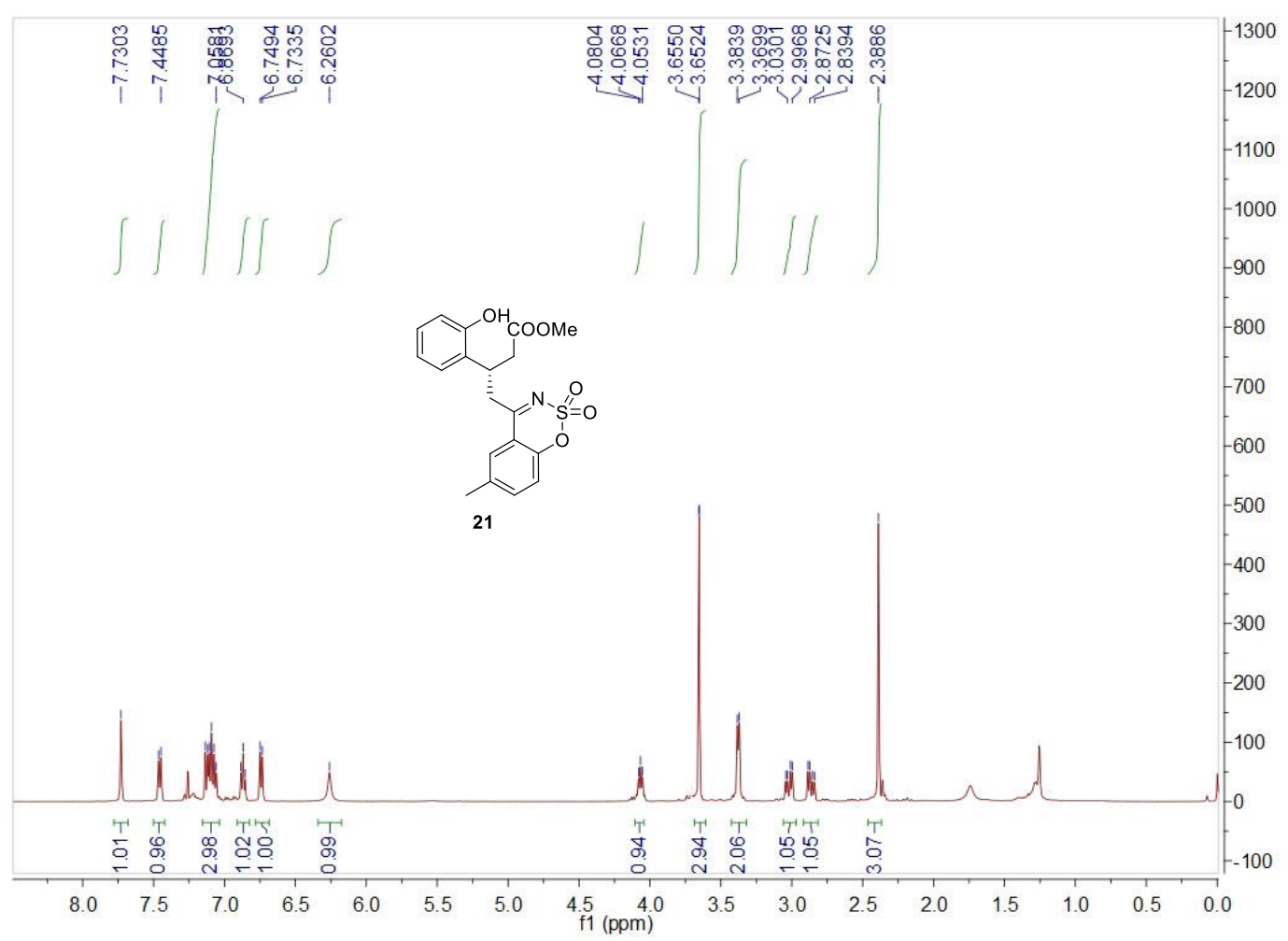

${ }^{13} \mathrm{C} \mathrm{NMR}$ of $\mathbf{2 1}, \mathrm{CDCl}_{3}$

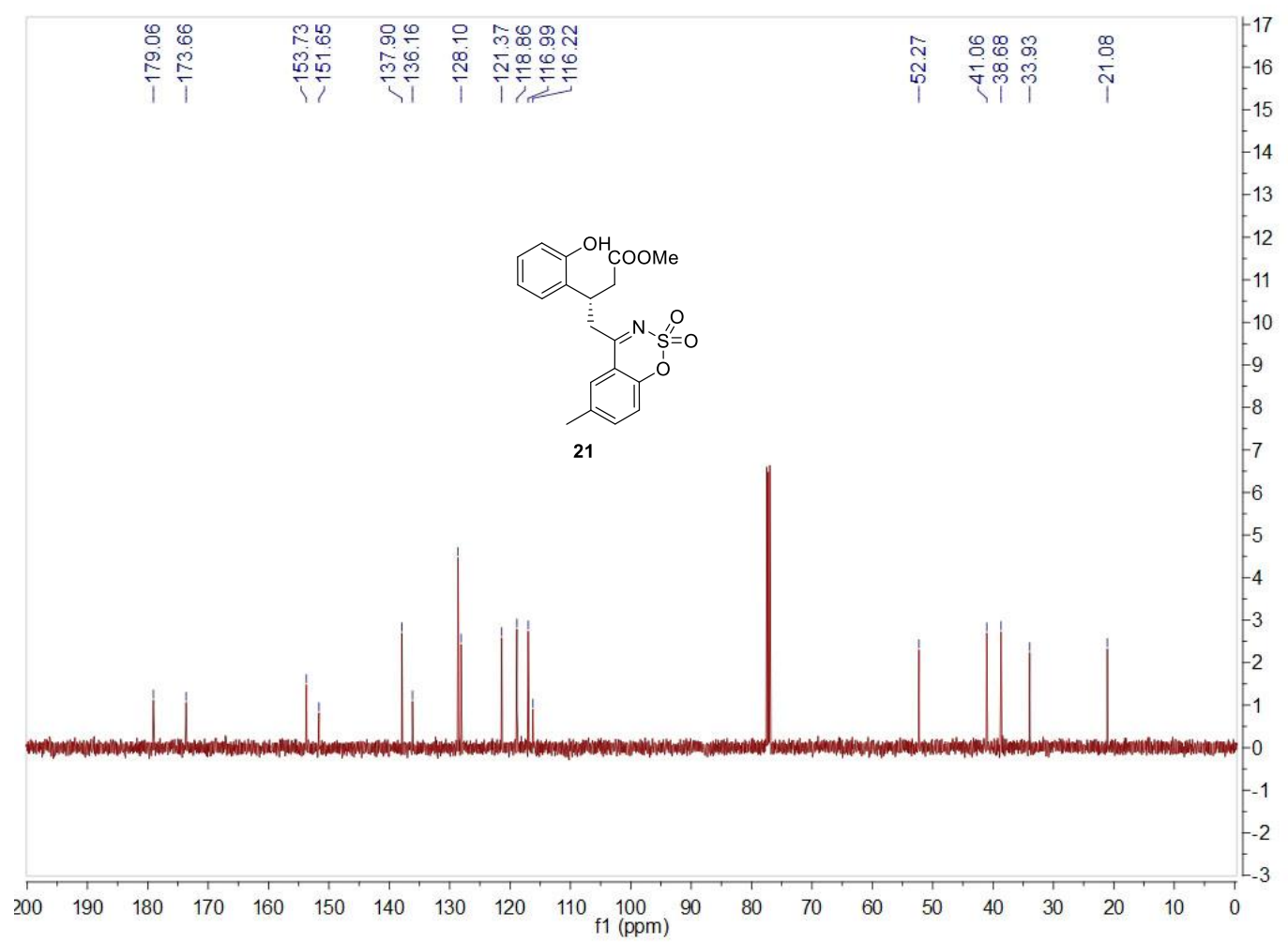

